Florida International University FIU Digital Commons

$3-25-2013$

\title{
Graphene NanoPlatelets Reinforced Tantalum Carbide consolidated by Spark Plasma Sintering
}

Andy Nieto

Florida International University, andynieto15@hotmail.com

DOI: $10.25148 /$ etd.FI13042212

Follow this and additional works at: https://digitalcommons.fiu.edu/etd

\section{Recommended Citation}

Nieto, Andy, "Graphene NanoPlatelets Reinforced Tantalum Carbide consolidated by Spark Plasma Sintering" (2013). FIU Electronic Theses and Dissertations. 840.

https:// digitalcommons.fiu.edu/etd/840

This work is brought to you for free and open access by the University Graduate School at FIU Digital Commons. It has been accepted for inclusion in FIU Electronic Theses and Dissertations by an authorized administrator of FIU Digital Commons. For more information, please contact dcc@fiu.edu. 


\title{
FLORIDA INTERNATIONAL UNIVERSITY \\ Miami, Florida
}

\section{GRAPHENE NANOPLATELETS REINFORCED TANTALUM CARBIDE CONSOLIDATED BY SPARK PLASMA SINTERING}

\author{
A thesis submitted in partial fulfillment of the \\ requirements for the degree of \\ MASTER OF SCIENCE \\ in \\ MATERIALS SCIENCE AND ENGINEERING \\ by \\ Andy Nieto
}

2013 
To: Dean Amir Mirmiran

College of Engineering and Computing

This thesis, written by Andy Nieto, and entitled Graphene Nanoplatelets Reinforced Tantalum Carbide Consolidated by Spark Plasma Sintering, having been approved in respect to style and intellectual content, is referred to you for judgment.

We have read this thesis and recommend that it be approved.

William Kinzy Jones

Norman Munroe

Arvind Agarwal, Major Professor

Date of Defense: March 25, 2013

The thesis of Andy Nieto is approved.

Dean Amir Mirmiran
College of Engineering and Computing

Florida International University, 2013 


\section{DEDICATION}

I dedicate this Thesis to life itself - for all the joys, adventures, wonders, and mysteries it provides, which have made life so enjoyable and worth living to the fullest. 


\section{ACKNOWLEDGEMENTS}

I would like to acknowledge and thank all the individuals who contributed in any way, shape, or form to this Thesis. First and foremost, I would like to thank my parents for all the love and care they have provided me my entire life. Loving parents set the foundation for a strong education and a successful life. I would like to especially thank my dad for bringing my brother and I to this wonderful country. The opportunities here abound and because of his hard work throughout his life we can reap the benefits of this great society. Our success is his source of joy and pride and that is the greatest motivation.

I would like to thank my girlfriend, Richa, for making the past year and a half so especially enjoyable and memorable. Finding someone so similar to me had always seemed like a fairy tale dream.

I'd like to thank my brother for all the great memories he provided me during our childhood and also for providing me a firsthand example of how success is achieved through education and hard work.

I would like to thank all the members of our research group at FIU for all their valuable mentoring and advice, in particular Drs. Arvind Agarwal and Debrupa Lahiri. Dr. Agarwal, through example has shown what it means to be a mentor and a researcher. Debrupa is a great friend and mentor, I remember her most for her love of science and her enduring and enthusiastic encouragement. Both of them exemplify how rewarding being a scientist can be, as when you do something you love, you never work a day in your life. 


\section{ABSTRACT OF THE THESIS \\ GRAPHENE NANOPLATELETS REINFORCED TANTALUM CARBIDE CONSOLIDATED BY SPARK PLASMA SINTERING}

by

Andy Nieto

Florida International University, 2013

Miami, Florida

\section{Professor Arvind Agarwal, Major Professor}

Hypersonic aerospace vehicles are severely limited by the lack of adequate high temperature materials that can withstand the harsh hypersonic environment. Tantalum carbide $(\mathrm{TaC})$, with a melting point of $3880^{\circ} \mathrm{C}$, is an ultrahigh temperature ceramic (UHTC) with potential applications such as scramjet engines, leading edges, and zero erosion nozzles. However, consolidation of $\mathrm{TaC}$ to a dense structure and its low fracture toughness are major challenges that make it currently unviable for hypersonic applications. In this study, Graphene NanoPlatelets (GNP) reinforced TaC composites are synthesized by spark plasma sintering (SPS) at extreme conditions of $1850^{\circ} \mathrm{C}$ and $80-100$ MPa. The addition of GNP improves densification and enhances fracture toughness of TaC by up to $\sim 100 \%$ through mechanisms such as GNP bending, sliding, pull-out, grain wrapping, crack bridging, and crack deflection. Also, TaC-GNP composites display improved oxidation behavior over $\mathrm{TaC}$ when exposed to a high temperature plasma flow exceeding $2500{ }^{\circ} \mathrm{C}$. 


\section{TABLE OF CONTENTS}

CHAPTER

PAGE

CHAPTER I: INTRODUCTION 1

1. Motivation 1

1.1. Need for High Performance Aerospace Materials 1

1.2. Impact of Advanced Aerospace Materials on Society 6

2. Research Objectives 10

2.1. Improved Mechanical Properties 11

2.2. Retention of UHTC Oxidation Behavior 12

CHAPTER II: REVIEW OF THE STATE OF THE ART 14

1. Ultra High Temperature Ceramics (UHTC) 14

1.1. Properties 14

1.2. Processing 21

2. Graphene 31

3. Carbon Allotrope Reinforced Composites 41

3.1. CNT Reinforced Composites 42

3.2. GNP Reinforced Composites 47

3.3. GNP vs. CNT 53

4. Spark Plasma Sintering 56

5. UHTC Oxidation Studies and Methods 63

5.1. Conventional Furnace Testing 64

5.2. Aerothermal Testing 68

CHAPTER III: $\quad$ EXPERIMENTAL METHODS 76

1. TaC-GNP Composite Powder Processing 76

$\begin{array}{ll}\text { 1.1. Wet Chemistry } & 78\end{array}$

1.2. High Energy Ball Milling $\quad 80$

2. Spark Plasma Sintering Processing 82

3. Structural Characterization 85

4. Mechanical Properties Characterization 86

\begin{tabular}{ll} 
4.1. & Microhardness \\
\hline
\end{tabular}

4.1.1. Low Load Testing $\quad 87$

4.1.2. High Load Testing $\quad 87$

4.2. Nano-Indentation 88

5. TaC-GNP Oxidation Behavior $\quad 89$

5.1. Plasma Jet Testing $\quad 89$

5.2. Characterization of the oxide 92

CHAPTER IV: RESULTS AND ANALYSIS 94

1. TaC-GNP Powder Characterization 94 
1.1. Homogeneity and Dispersion of GNP 94

1.1.1. Powder Processed by Wet Chemistry Only 95

1.2.1. High Energy Ball Milled Powder 99

1.2. Structure of GNP 102

1.2.1. Structure after ultrasonication 102

1.2.2. Structure after attrition milling 103

2. Synthesis of Bulk GNP 105

2.1. Bulk Structure Consolidation 105

2.2. Bulk GNP Microstructure 107

3. Consolidation of TaC-GNP Composites 110

4. TaC-GNP Composites 114

4.1. Microstructure 114

4.2. Phases in SPS TaC-GNP Composites 124

5. Mechanical Properties of TaC-GNP Composites 129

5.1. Elastic Modulus 129

5.2. Hardness 133

5.3. Fracture Toughness 137

5.4. Toughening Mechanisms 139

5.4.1. Toughening Mechanism in Bulk GNP 139

5.4.2. Toughening in TaC-GNP Composites 141

6. TaC-GNP Oxidation Behavior 145

6.1. Oxidation of TaC-GNP Composites 145

6.2. Oxidation Mechanisms and Microstructural evolution 148

6.2.1. TaC-GNP Thermal Conductivity 149

6.2.2. TaC-GNP Microstructural Mechanisms $\quad 152$

6.2.3. GNP Induced Chemical Reactions 157

6.2.4. Oxidation behavior variations in TaC-GNP 163

6.3. GNP Structure 165

$\begin{array}{ll}\text { CHAPTER 5: CONCLUSIONS } & 171\end{array}$

CHAPTER 6: FUTURE RESEARCH 173

$\begin{array}{ll}\text { LIST OF REFERENCES } & 174\end{array}$ 


\section{LIST OF TABLES}

TABLE

PAGE

Table 3.1: $\quad$ TaC-GNP composite powder compositions 77

Table 4.1: $\quad$ Summary of Raman Spectrum Peak Ratios for Wet Chemistry 101 and Ball Milling Processed Powders

Table 4.2: $\quad$ Summary of Consolidated TaC-GNP Samples Densification $\quad 110$

Table 4.3: $\quad$ Grain Size and Densification Summary 117

Table 4.4: $\quad$ Summary of GNP dispersion and effect on microstructure 123

Table 4.5: $\quad$ Summary of TaC-GNP Powder and SPS Compact 125

Compositions as determined from peak areas in XRD Patterns

Powders and SPS Samples

Table 4.7: $\quad$ Summary of nanoindentation results of TaC-GNP samples 130

Table 4.8: $\quad$ Summary of TaC-GNP Oxidized samples and SPS Compact 147

Table 4.9: $\quad$ Summary of Raman Spectrum Peak Ratios for TaC-GNP Oxidized Samples and SPS Samples 


\section{LIST OF FIGURES}

FIGURE

PAGE

Figure 1.1: a) Saturn V F-1 Rocket Engine b) NASA DM-3 Rocket Engine Test Firing

Figure 1.2: a) SCRAMjet Engine Schematic b) HIFIRE SCRAMjet engine Test Firing

Figure 1.3 : a) Apollo Program Reentry Capsules b) Space Shuttle Landing

Figure 1.4: a) Boeing X-51 Waverider b) NASA X-43 9

Figure 2.1: $\quad$ TaC Phase Diagram 20

Figure 2.2: Hypersonic leading edge heat transfer 30

Figure 2.3: a) Graphene honeycomb structure b) TEM of graphene layers 31

Figure 2.4: Graphene Raman Spectra 38

Figure 2.5: a) Graphene grown on $\mathrm{SiC}$ substrate with cavities b) AFM 40

Figure 2.6: $\quad$ CNT Crack Bridging in CNT reinforced Hydroxyapatite composite 45

Figure 2.7: $\quad$ CNT embedded in Tantalum Carbide grain 46

Figure 2.8: $\quad$ GNP crack bridging by a) large sized GNP $(\sim 6 \mu \mathrm{m})$ and b) small 51 sized GNP ( 200 nm), in GNP reinforced Si3N4 composites

Figure 2.9: GNP induced crack branching in GNP reinforced Si3N4 composite 52

Figure 2.10: TEM micrograph of GNP wrapped around Si3N4 grain 52

Figure 2.11: Heat transfer mechanisms present due to pulsing electric current 58 during spark plasma sintering

Figure 2.12: Schematic of Spark Plasma Sintering machine 60

Figure 2.13: Geometries used in UHTC Aerothermal oxidation studies, a) Flat 69 Thin Bar, b) Streamlined Flat Plate, c) Blunt Body, d) Sharp Cone

Figure 2.14: a) X-52 Waverider leading edge, b) Sharp Thin Wedge geometry used 70 for UHTC aerothermal oxidation studies

Figure 2.15: Post high temperature oxidation structure of a) $\mathrm{Si} 3 \mathrm{~N} 4-\mathrm{MoSi}_{2}$ b 3.4) $\mathrm{ZrB}_{2}-\mathrm{SiC}$ composites 
Figure 2.16: $\mathrm{ZrB}_{2}-\mathrm{SiC}$ microstructure near sharp cone leading edge after oxidation in high temperature Mach 3 plasma flow

Figure 3.1: $\quad$ SEM of Tantalum Carbide $(\mathrm{TaC})$ as received powder from Inframet Advanced Materials, a) low magnification and (b) high magnification

Figure 3.2: a) SEM of Graphene NanoPlatelets (GNP) as received powder from XG Sciences, b) top view and (b) stack of few GNPs in cross-section

Figure 3.3: Typical morphology of TaC-GNP powders after evaporation of ultrasonication medium - acetone

Figure 3.4: a) High Energy Ball Mill used to process TaC-GNP composite powders, b) Stainless Steel Jar and Balls used for ball milling

Figure 3.5: SPS Processing Charts; a) TaC-1G, b) TaC-2G, c) TaC-3G,

d) $\mathrm{TaC}-4 \mathrm{G}, \mathrm{e}) \mathrm{TaC}-5 \mathrm{G}, \mathrm{f}$ ) Tantalum Carbide (TaC), g) Graphene NanoPlatelets (GNP)

Figure 3.6: TaC-GNP Oxidation Test Sample cut out from SPS Compacts

Figure 3.7: $\quad$ Tungsten Fixture for holding specimens during Plasma Jet Oxidation; a) Backside View, b) Side View

Figure 3.8: $\quad$ Plasma Jet Oxidation Testing Experimental Setup

Figure 4.1: TaC-1G Powder processed by Wet Chemistry a)Low Mag,

b) High Mag

Figure 4.2: $\quad$ Transparent GNP in TaC-1G Powder 95

Figure 4.3: $\quad$ TaC-3G Powder Processed by Wet Chemistry 96

Figure 4.4: $\quad$ TaC-3G Powder, a) Transparent GNP, b) Wrinkled GNP 96

Figure 4.5: $\quad$ Segregated GNPs in TaC-5G Powder 97

Figure 4.6: $\quad$ Smaller $(3-5 \mu \mathrm{m})$ GNPs in TaC-5G Powder showing minimal 98

Figure 4.7: Crumpled GNP alongside transparent GNP in TaC-5G Powder 98

Figure 4.8: $\quad$ TaC-5G Powder - region with improved GNP dispersion 99 
Figure 4.9: Ball Milled TaC-1G, a) Minimal presence of GNPs is observed,

b) Small flakes lie on $\mathrm{TaC}$ particles, and c) in between $\mathrm{TaC}$ particles

Figure 4.10: Ball Milled TaC-5G Powder, several GNP flakes can be seen

Figure 4.11: Ball Milled TaC-5G Powder, a) Few large GNPs have survived,

Figure 4.12: Raman Spectra of a) TaC-1G, b) TaC-3G, and c) TaC-5G Powders 101

Figure 4.13: SPS processing cycle used to consolidate the bulk GNP sample $\quad 105$

Figure 4.14: $\quad$ Bulk GNP synthesized by SPS Processing, penny shown for size 106 Comparison

Figure 4.15: $\quad$ SEM images of consolidated GNP structure's fracture surface reveal stacks of uniformly oriented GNP layers at (a) low magnification and (b) higher magnification. Carbon tape used to hold sample for SEM is seen near top and bottom of image in Fig. 4.15a.

Figure 4.16: SEM images of consolidated GNP structure: a) Top Surface showing minor damage at edges and b) transverse surface showing highly oriented structure.

Figure 4.17: Micro Raman Spectroscopy of starting GNP Powder and GNP SPS Pellet

Figure 4.18: Typical Images of Consolidated TaC-GNP samples, a) TaC,

b) $\mathrm{TaC}-1 \mathrm{G}, \mathrm{c}) \mathrm{TaC}-3 \mathrm{G}$, d) $\mathrm{TaC}-5 \mathrm{G}$, e) Thinnest Sample - TaC, f) Thickest Sample - TaC-3G

Figure 4.19: Optical Micrographs of a) TaC, b) TaC-1G, c) TaC-3G,

d) $\mathrm{TaC}-5 \mathrm{G}$

Figure 4.20: Plot of Densification (relative to theoretical density) vs. GNP

Content. Densification can be seen to increase with increasing GNP content

Figure 4.21: TaC Fracture Surface displays two distinct microstructure, a) Consolidated Regions, b) Porous poorly sintered regions

Figure 4.22: $\quad$ TaC-1G fracture surface showing less poorly sintered regions and a more homogenous microstructure 
Figure 4.23: Grain Size vs. GNP Content. Grain Size can be seen to decrease with increasing GNP content

Figure 4.24: a) b) TaC-1G Fracture Surfaces with several embedded GNP,

c) High mag image

Figure 4.25: Varying Interactions of GNP with $\mathrm{TaC}$ in $\mathrm{TaC}-1 \mathrm{G}$, a) Embedded GNPs, c) GNP around TaC grains

Figure 4.26: GNP Distribution in TaC-3G, a) ,b) GNPs form continuous network, c) High Mag image of GNPs extending across several TaC grains, d) Two GNP meeting and forming coherent interface - this allows for formation of continuous GNP networks

Figure 4.27: TaC-3G Fracture Surface: GNP preferred orientation observed 120 perpendicular to SPS Pressing axis, GNPs of various sizes are Observed

Figure 4.28: TaC-5G Fracture Surface showing larger GNPs, a) Arrows denote gaps b) Gaps present at interface of large GNPs with TaC grains, c) High magnification of wrinkles and folds in large GNP

Figure 4.29: a) GNP wrapped around multiple $\mathrm{TaC}$ grains in $\mathrm{TaC}-5 \mathrm{G}$,

b) GNP weaving around $\mathrm{TaC}$ grains in $\mathrm{TaC}-5 \mathrm{G}$, some embedded GNPs can be seen

Figure 4.30: X-Ray Diffractions Patterns of TaC Powder and SPS TaC-GNP Compacts. All major peaks visible correspond to $\mathrm{TaC}$

Figure 4.31: Raman Spectra of Powders and SPS Compacts a) TaC-1G,

b) $\mathrm{TaC}-3 \mathrm{G}$, and c) $\mathrm{TaC}-5 \mathrm{G}$

Figure 4.32: Elastic Modulus values measured by nanoindentation for TaC-GNP Samples

Figure 4.33: Characteristic Load-Displacement curves during nanoindentation of TaC-GNP samples

Figure 4.34: 3D profile of typical Nano-indent (Generated by SPM)

Figure 4.35: GNP in between Grains, a) GNP deforming and shearing off, 132 b) GNP causing localized porosity in between $\mathrm{TaC}$ grains 
Figure 4.36: Nano-Hardness of TaC-GNP samples, measured by

Figure 4.37: Schematic illustrating how GNP can have an increased effect on hardness when indenting on a single grain as compared to an indentation spanning many $(>30)$ grains

Figure 4.38: MicroHardness comparison across different indentation loads

Figure 4.39: Fracture Toughness of TaC-GNP samples

Figure 4.40: Optical micrographs of micro-indents in a)TaC, b) TaC-5G;

Figure 4.41: a) Bending at high angles observed across multiple stacks of GNPs, b) Bending at high angles observed in single stack of GNP, c) in-plane view of bent GNPs, d) Bending of multiple GNP stacks, with some stacks becoming offset because of the interlayer sliding. No fracture is observed at high bending angles indicating high strength and flexibility of GNPs

Figure 4.42: GNP structures in TaC-GNP fracture surfaces, a) GNP displaying significant kinks and bends, b) High magnification image showing multiple GNP stacks, kinking c) Individual sheets in a GNP undergoing sliding

Figure 4.43: a) GNP pulled out of grain, b) GNP top surface spanning TaC grains suggests GNP grain wrapping, Inset shows GNP wrapping in $\mathrm{TaC}-5 \mathrm{G}$ fracture surface

Figure 4.44: a) GNP stacks sliding and deflecting a crack, b) Multiple single GNP bridging a crack, c) GNP twisting as it bridges a crack, d) Multiple Bundled GNP bridging a crack

Figure 4.45: TaC-GNP samples post plasma flow oxidation, a) TaC, b) $\mathrm{TaC}-1 \mathrm{G}$, ) TaC-3G, d) TaC-5G, e) Typical Interface showing blue oxide from Tungsten Fixture

Figure 4.46: X-Ray Diffractions Patterns of Oxidized TaC-GNP samples

Figure 4.47: TaC-GNP oxidized samples oxide layer thickness, inset images show optical micrographs of $\mathrm{TaC}$ and $\mathrm{TaC}-3 \mathrm{G}$ oxide layers

Figure 4.48: Thermal Gradient experienced by TaC-GNP samples during 60 second plasma flow exposure 
Figure 4.49: Schematic illustrating effect of GNP on heat transfer

throughout sample

Figure 4.50: a) Typical structure of TaC-GNP unoxidized surface, b) High magnification of unoxidized top surface, c) Oxidized top surface of $\mathrm{TaC}, \mathrm{d})$ High magnification of oxidized $\mathrm{TaC}$

Figure 4.51: a) Chasm in $\mathrm{TaC}$ oxidized sample as a result of gases bursting out of the sample, b) Underlying layer showing pores caused by gases seeping out before accumulating and bursting through the top layer

Figure 4.52: TaC-GNP composites oxidized top surface a) Typical structure of oxidized top surfaces, b) Structure reveals large cracks but no large chasms, c) Areas with large cracks are peeling outwards but have not burst out, d) Edge of 'burst' region reveals elongated grain structure

Figure 4.53: Hardness of TaC-GNP oxide layers, tougher oxide layers experience less damage allowing them to better resist permanent deformation

Figure 4.54: Schematic illustrating effect of GNP on oxygen diffusion into sample

Figure 4.55: EDS Analysis of TaC-GNP samples, a)TaC, b) TaC-1G, c) TaC-3G, d) TaC-5G, EDS results for nitrogen are omitted as no change due to GNP composition was observed

Figure 4.56: Typical Oxidized Cross-sections in which EDS analysis was performed, a) $\mathrm{TaC}$, b) $\mathrm{TaC}-1 \mathrm{G}$, c) $\mathrm{TaC}-3 \mathrm{G}$, d) $\mathrm{TaC}-5 \mathrm{G}$

Figure 4.57: EDS Analysis of oxidized TaC-3G cross-section at interface 160 between oxide and non-oxide, a) Backscattered Image of EDS Analysis location, b) Carbon x-ray map, c) Oxygen x-ray map, d) Tantalum x-ray map

Figure 4.58: EDS Analysis of oxidized TaC cross-section at interface between 160 oxide and non-oxide, a) Backscattered Image of EDS Analysis location, b) Carbon x-ray map, c) Oxygen x-ray map, d) Tantalum x-ray map 
Figure 4.59: EDS Analysis of oxidized TaC - $1 \mathrm{G}$ cross-section at interface between oxide and non-oxide, a) Backscattered Image of EDS Analysis location, b) Carbon x-ray map, c) Oxygen x-ray map, d) Tantalum x-ray map

Figure 4.60: EDS Analysis of oxidized TaC-5G cross-section at interface between oxide and non-oxide, a) Backscattered Image of EDS Analysis location, b) Carbon x-ray map, c) Oxygen x-ray map, d) Tantalum x-ray map

Figure 4.61: Surface Recession of TaC-GNP after 60 second oxidation across compositions. Percentage is relative to original sample thickness

Figure 4.62: Raman Spectra of Oxidized Samples and SPS Compacts 166 a) $\mathrm{TaC}-1 \mathrm{G}, \mathrm{b}) \mathrm{TaC}-3 \mathrm{G}$, and c) $\mathrm{TaC}-5 \mathrm{G}$

Figure 4.63: Carbon structures in Oxidized samples, a) TaC-1G top surface showing possible agglomerated GNP, b) TaC-5G top surface showing folded and multiple platelet GNP, c) Embedded Carbon in TaC-3G top surface, d) Tubular carbon 'band' present in TaC-3G top surface

Figure 4.64: Carbon bands in oxide structure, a) Carbon band in TaC-1G appears to be wetted by the TaC based oxide, b) Smooth GNP embedded in TaC-3G oxide, c) Carbon band in TaC-5G cross section, d) Wetted band spanning $100 \mathrm{~nm}$

Figure 4.65: Large surviving GNP in oxide structure, a) GNP structure likely consisting of several platelets joined edge-to-edge as a result of high temperature, b) Thin $\sim 15$ micron GNP showing smooth surface with little damage 


\section{CHAPTER I: INTRODUCTION}

Emerging technologies in the aerospace field require materials with the capacity to survive at extreme temperatures and conditions. Ultra high temperature ceramics (UHTC) have been touted as a possible solution because of their high resistance to oxidation and chemical attack, along with their impressive mechanical properties. $\mathrm{TaC}$ is an ultra-high temperature ceramic which has one of the highest melting points of any material along with excellent mechanical properties. Challenges to implementing $\mathrm{TaC}$ in aerospace systems include the bulk consolidation of these materials without sacrificing structural and mechanical integrity. An inherent challenge in using $\mathrm{TaC}$ is that is it a ceramic and hence very brittle. The improvement of fracture toughness is crucial to ensuring the high structural performance during service. The addition of Graphene Nanoplatelets has shown to toughen ceramics and thus TaC-GNP composites may provide a solution to the toughening problem. The addition of GNP must not have adverse effects on oxidation behavior - potential for enhancement in oxidation behavior due to GNP addition is possible due to high thermal conductivity of GNP.

\section{$\underline{\text { 1. Motivation }}$}

\subsection{Need for High Performance Aerospace Materials}

The central motivation of this Thesis lies in the need for high performance aerospace materials that are needed for the next generation of aerospace systems. Aerospace engineering is often said to be driven towards designing systems to go higher, faster, and further. Aerospace systems, like all engineering systems are ultimately limited by the capacities of the materials available. A prime example of an aircraft's failure due to 
inadequate materials is the test flight of the hypersonic Falcon HTV-2 which according to DARPA crashed into the ocean after "unexpected aeroshell degradation" due to excessive thermal and structural loads from the brutal shock waves present at such high Mach numbers [1]. Many current aerospace concepts cannot be realized without the emergence of new advanced high performance materials. New materials may also inspire new innovations and concepts that would be impossible with currently available materials.

Hypersonic flight is currently one of the areas of aerospace engineering that is generating the most interest. Hypersonic flight is defined as flight above Mach 5, which is 5 times the speed of sound. Hypersonic flight has only been previously achieved by spacecraft during atmospheric escape and planetary entry. The next generation of hypersonic vehicles will be designed for either atmospheric flight plans or deep space missions. Extremely high temperatures $\left(>2000{ }^{\circ} \mathrm{C}[2]\right)$ are experienced by hypersonic spacecraft and aircrafts. Hypersonic vehicles of the future will require highly efficient engines capable of reaching high hypersonic speeds. Engines capable of these velocities are typically rocket or SCRAMjet engines. Typical rocket and SCRAMjet engines are shown in Fig. 1.1 and Fig. 1.2 respectively. A Rocket engine requires onboard sources of oxygen for the combustion cycle to be completed while SCRAMjet engines are airbreathing engines. Rocket and SCRAMjet engines like other combustion engines are limited by thermodynamic efficiency. Rockets have been optimized for decades to reach very high thermodynamic efficiencies. SCRAMjets have been developed more recently but their thermodynamic efficiencies have also neared their limit with the currently available technology. The thermodynamic performance can be improved by increasing 

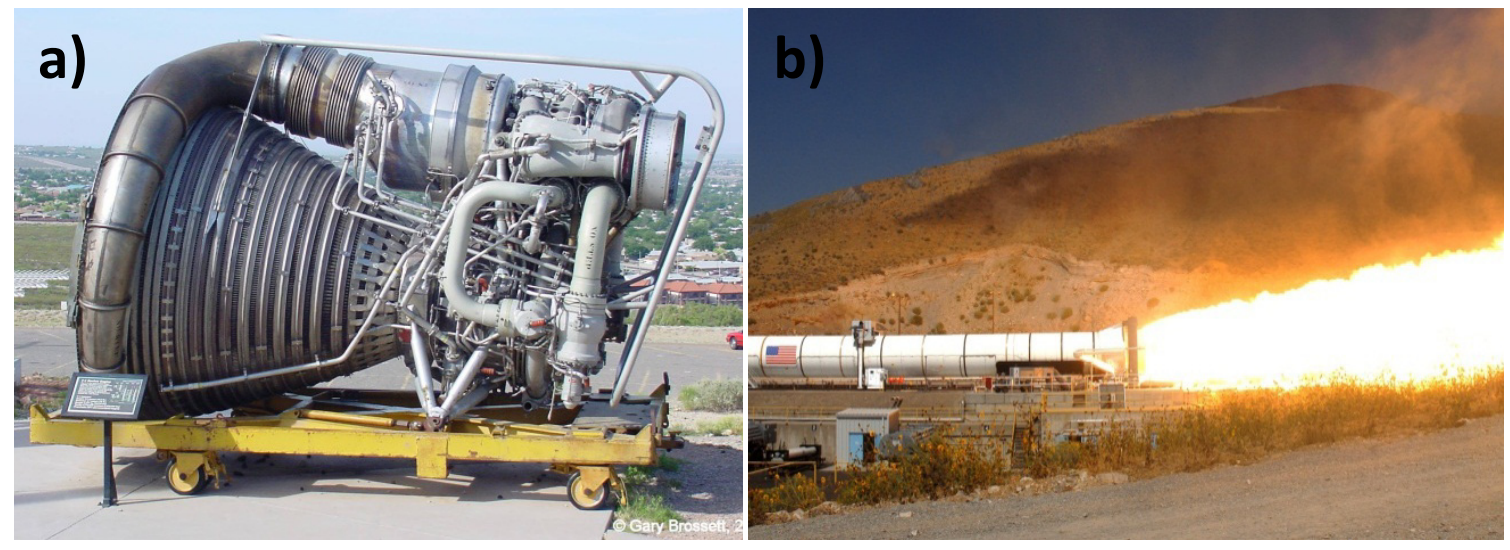

the working temperature, but this maximum temperature is limited by the materials used. If the material cannot withstand the high temperatures generated by the engine then the system will fail. Higher temperatures in the engine require new materials that can withstand these temperatures and this is one of the main driving forces of high temperature materials engineering.

Another critical issue for future hypersonic vehicles will be the high temperatures experienced on the exterior as a result of aerothermal heating. Previous hypersonic
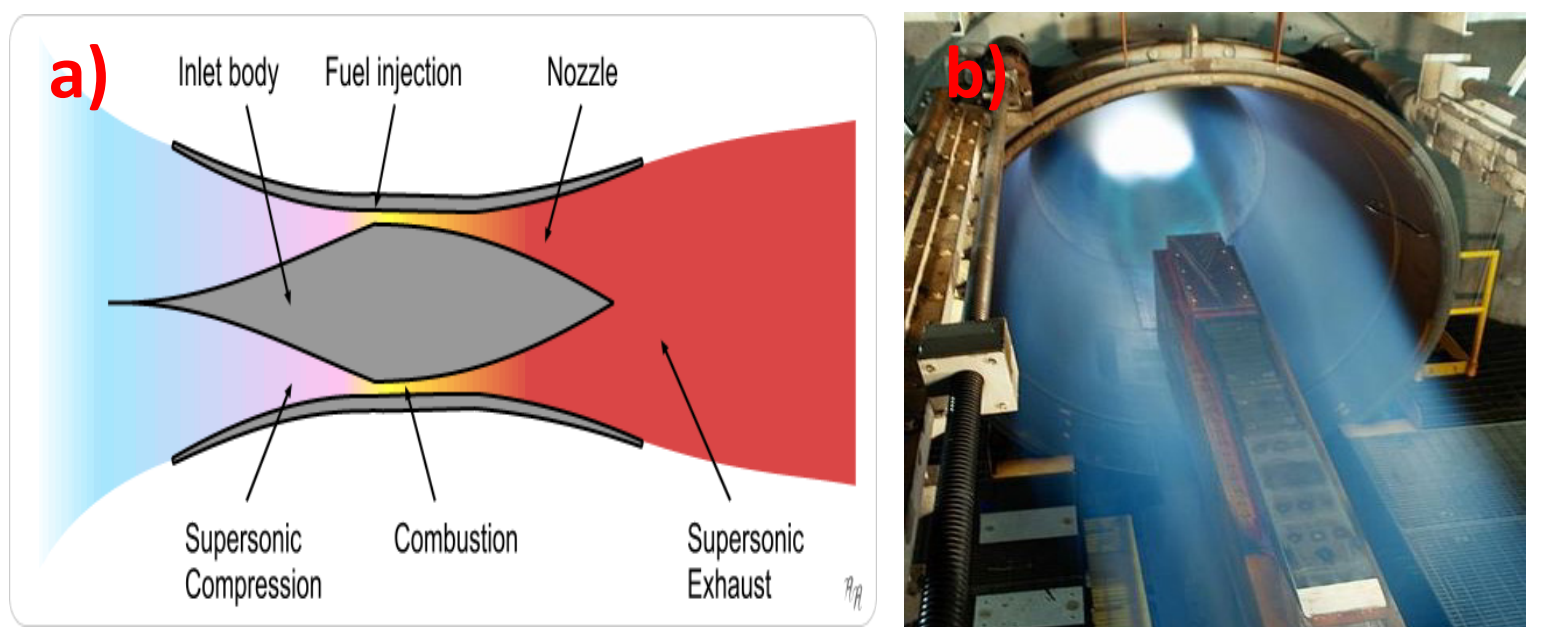
spacecraft such as the Apollo Program reentry capsules and the Space Shuttles, shown in Fig. 1.3, had blunt bodies as leading edges, which resulted in lower heat fluxes. Aerothermal heating will be a minor issue for spacecraft as most designs for future deep space missions retain the blunt body shape. These blunt bodies however generate large amounts of drag and thus have low aerodynamic efficiency (i.e. Lift divided by Drag). However, aircraft that are designed to operate in the atmosphere (e.g. spaceplanes, drones, missiles) need high aerodynamic efficiency and thus blunt bodies are unsuitable for hypersonic atmospheric flight. Hypersonic spaceplanes will be subjected to high aerothermal loads. The leading edges will be sharp (low radius of curvature) and slender in order to achieve high aerodynamic efficiency. High aerodynamic efficiency enables the aircraft to have high maneuverability and control thus increasing safety and flight performance. The low radius of curvature at the leading edge will lead to temperatures above $2000{ }^{\circ} \mathrm{C}$ at the stagnation point of the aircraft. These vehicles will require high temperature materials at the stagnation point and the surrounding area in order to maintain structural integrity.

High temperature materials are needed for both hypersonic vehicle propulsion systems and leading edges. Although these are two very different parts of the aircraft, the material requirements are very similar. High temperature resistance is needed while maintaining a level of structural stability. The environment present at leading edges and propulsion systems will also be subjected to chemical attack, corrosion, and oxidation [3]. In propulsion systems, the materials will be subjected to combustion products of the fuel. Certain engine concepts also employ ion-engine concepts and the material will thus 

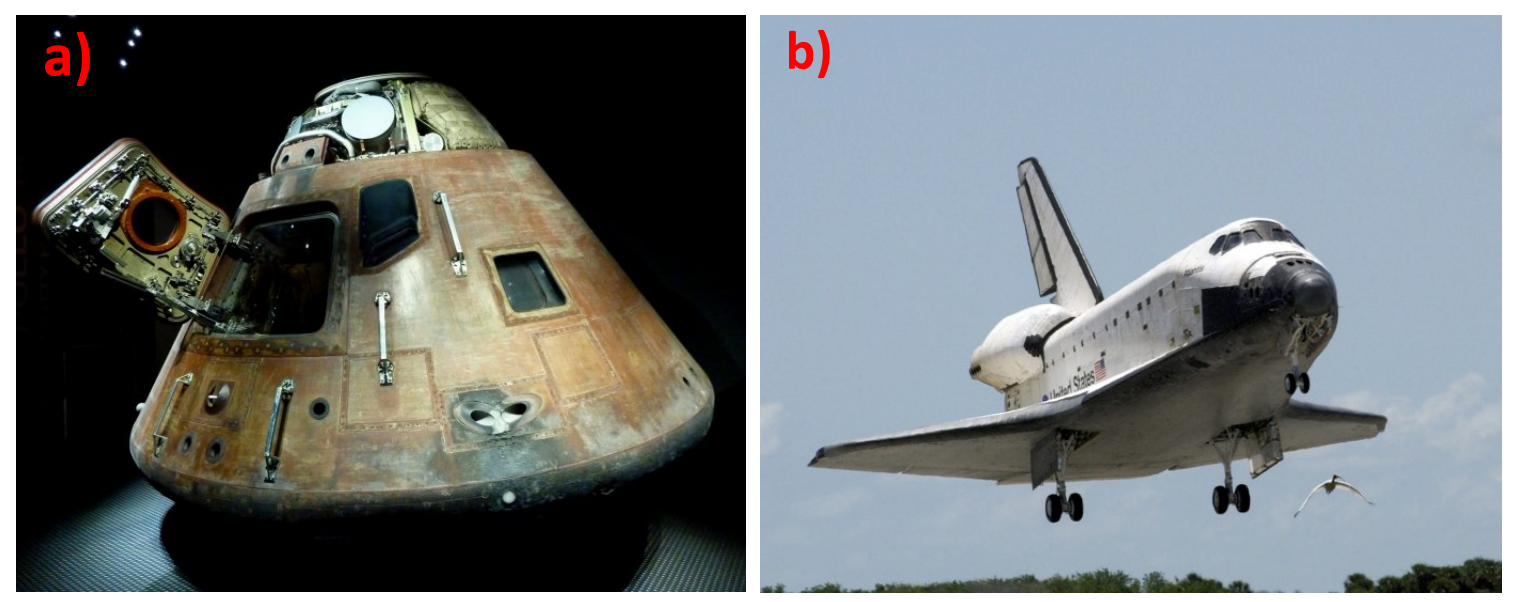

be subjected to an electrical discharge as well. At very high temperatures the air itself will begin to dissociate, first oxygen as its bond dissociation energy (BDE) is 498.40 $\mathrm{kJ} / \mathrm{mole}$ [4], and then Nitrogen whose BDE is $945.42 \mathrm{~kJ} / \mathrm{mole}$ [4]. Ionized Nitogen and Oxygen atoms are highly reactive and therefore lead to highly oxidizing environments. High temperature materials should therefore also have substantial oxidation resistance and strength.

Hypersonic vehicles require the emergence of engineering technologies and innovations from various fields of engineering. Avionics and electronics have advanced to the point where systems (e.g. drones, UAVs) can be remotely operated $[5,6]$ or operated by virtual pilot technologies [7]. These technologies can be used to overcome the challenges of having windows in aircraft whose speeds and temperatures reach such high values. From an aerodynamics standpoint; designs are employing waverider concepts to increase aerodynamic efficiency and reduce wave drag at such high speeds. [8]. Variable geometry designs have been proposed for both supersonic and hypersonic systems $[9,10]$ which overcome the technical barrier of high aerodynamic efficiency at 
both subsonic and hypersonic speeds. Engines which can operate at both subsonic and hypersonic regimes are also being developed [11, 12]. Ultimately, the most critical piece of developing hypersonic vehicles is the development of materials can survive the extreme conditions experienced during hypersonic flight.

\subsection{Impact of Advanced Aerospace Materials on Society}

Both governments and private companies are pushing the future of aerospace engineering towards hypersonic flight in both atmospheric conditions and orbital or deep space environments. Private companies like SpaceX and Virgin Galactic have cropped up with ambitious plans for space tourism. These spacecraft need not go hypersonic speeds and the materials needed to produce them are presently available. These aircrafts however currently are designed for limited space exposures. As the space industry grows, more companies will appear and the competition among them will drive the designs towards higher performance and more ambitious flight plans. Vehicles will likely have to travel at hypersonic speeds to escape the atmosphere and also during atmospheric entry. Aside from touristic activities, these companies are also designing spacecraft to serve as the next generation of shuttles for astronauts traveling to the International Space Station (ISS) [13]. NASA's latest budgets have reflected the shift towards more reliability in private companies for access into space [14]. The impact of private access to space opens up economic and scientific opportunities alike. SpaceX currently has a $\$ 1.6$ billion contract to bring at least $20000 \mathrm{~kg}$ of supplies and equipment to the ISS over a minimum of 12 missions [15]. The contract is expandable to $\$ 3.4$ billion if more missions can be 
completed thus potentially increasing the longevity of the ISS. Space tourism has also brought attention to concepts once deemed pipe dreams. Companies are investigating the possibility of orbiting stations for touristic uses such as space hotels that would serve as destinations for space tourists.

Many scientists and engineers also envision hypersonic flight being available for civil transports. Currently there are no hypersonic or even supersonic civil transports in service. Commercial supersonic flight was previously achieved by the Concorde and the Tipolev Tu-144. Both of these aircraft however are no longer in service, the Tu-144 retired in 1978 and the Concorde in 2003. While supersonic aircrafts have flown previously both commercially and for military missions, hypersonic aircrafts are only in the early stages of design and testing. A hypersonic civil transport would travel at speeds about 3 times that of the Concorde and would therefore be able to take passengers between any two points on the Earth in a matter of a few hours. Such transportation capabilities would vastly enlarge and enhance global travel and economic opportunities. Commercial and military hypersonic vehicles offer the challenge of being durable and reusable. Hypersonic spacecraft such as Apollo Orbiters and Space Shuttles used ablative materials such as Phenolic Impregnated Carbon Ablator (PICA) [16] which could withstand the high temperatures for a few hundred seconds. Such materials would not suitable for hypersonic aircraft such as civil transports for two main reasons. Hypersonic aircraft would be expected to perform at hypersonic speeds for hours and not seconds thus greatly increasing the exposure time. Also, a civil transport would be expected to be robust enough to perform multiple flights in a day. The aircraft should not have their 
Thermal Protection System (TPS) evaporate as occurred with ablative systems in Apollo Orbiters or replaced as with the tiles used in space shuttles. The TPS of a hypersonic civil transport should be highly durable and reliable in order to perform multiple flights with relatively low inspection times.

As previously mentioned hypersonic spacecraft have previously flown in the form of space shuttles and lunar orbiters. The next generation of spacecraft will have longer and more ambitious missions to complete. Spacecraft concepts are being developed that can propel to asteroids and mars. Concepts are being developed to extract minerals from Asteroids either to bring back to Earth or to aid in developing bases on asteroids $[17,18]$. Asteroids may hold exotic minerals with many potential applications. Asteroids could also serve as launching pads to Mars. Mars missions involving manned and unmanned spacecraft are being designed with the goal of establishing Mars' past, present, and future potential for life. To achieve these missions in reasonable times the spacecrafts must achieve high escape velocities from the Earth. New engines are being designed that can produce the necessary thrust values needed to escape the Earth at a high enough velocities. These new engines are pushing the thermodynamic limits of rocket engines thus increasing the operating temperatures of these engines. These engines would not be reusable and thus not have the technical challenges of commercial spacecraft and aircraft from a durability standpoint. These engines would be some of the first next generation hypersonic vehicles to be manifested as the high temperature materials would not be expected to survive more than a few minutes as the engines could be abandoned upon escape from the atmosphere. 

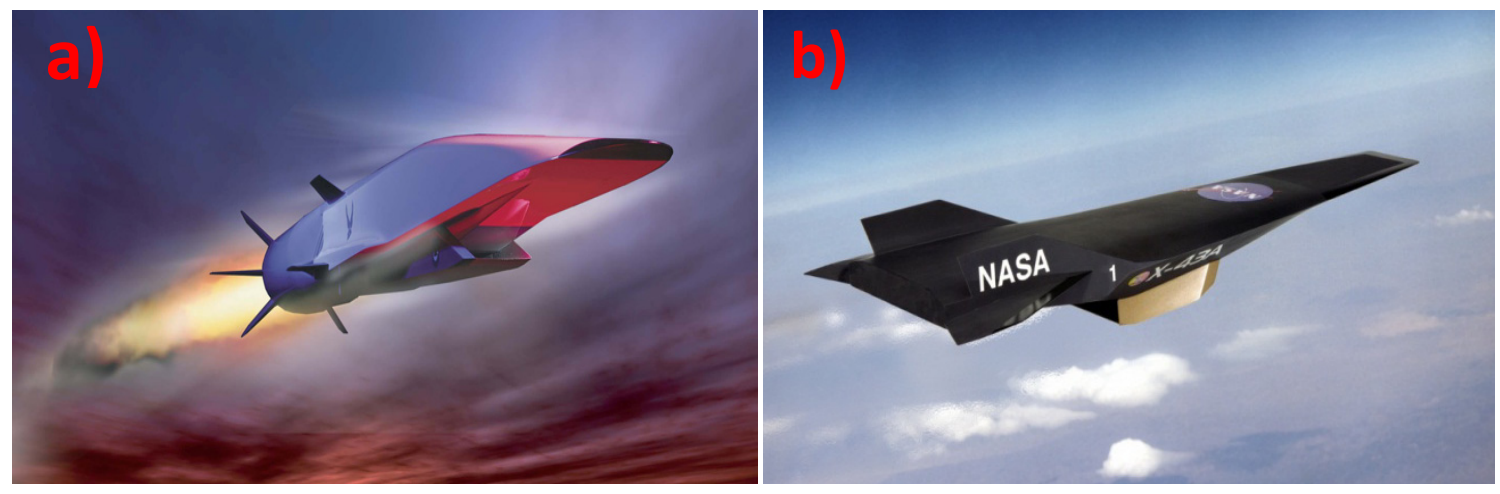

As with many engineering technologies, the first applications of hypersonic aircraft will likely be in military technologies. Many of the current hypersonic prototypes being developed would serve as platforms for hypersonic missiles. Missiles have fewer challenges as there are no requirements for passenger safety or reusability. Boeing's X-51 Waverider program and NASA's Hyper X-Program are developing vehicles that are taken to atmosphere by large subsonic carriers (e.g. B-52) and then dropped off where they fire their engines and reach hypersonic speeds $[19,20]$. Some of the prototypes developed by these programs are shown in Fig. 1.4. Hypersonic missiles can be optimized for hypersonic aerodynamic performance as they do not operate in the subsonic regimes. These missiles require high temperature materials for both the leading edges and the SCRAMjet engines that provide them with the necessary thrust for hypersonic speeds. Hypersonic missiles would provide the military with an edge in Cold War Era missile systems as well as modern day counter-terrorism operations. Hypersonic missiles would allow for the delivery of nuclear warheads within minutes to any nation on Earth thus enhancing the strength of a nation's nuclear deterrent. These missiles could also be used to carry out attacks on high profile terrorist targets in a matter of minutes 
before they move to another location. Currently, unless a drone is stationed nearby the target, it can take several hours for missiles launched from a nearby base or aircraft carrier to reach their target. The inherent large kinetic energy of hypersonic missiles would also increase their destructive power.

\section{Research Objectives}

The overall objective of this Thesis is to synthesize Graphene NanoPlatelets (GNP) reinforced Tantalum Carbide (TaC) composites with improved strength, fracture toughness, and oxidation behavior. The following points outline the approach taken to meet the objectives

- Effective use of Graphene NanoPlatelets as reinforcement necessitates homogeneous distribution of GNP into TaC matrix - Wet chemistry methods are employed to produce TaC-GNP powders efficiently and effectively.

- $\mathrm{TaC}$ based composite require high densification for achievement of high mechanical properties - Spark Plasma Sintering is utilized to consolidate TaCGNP composites in order to high densification without adverse grain growth effects.

- $\quad$ Structure retention of GNP during Spark Plasma Sintering processing is evaluated through synthesis of Bulk GNP. 
- Effects of GNP on strengthening and toughening of TaC-GNP composites is evaluated through micro- and nano-indentation experiments - Microstructural observation used to elucidate GNP strengthening and toughening mechanisms.

- Implementation of TaC-GNP composites in aerospace systems requires survival under extreme temperatures $-T a C$-GNP composites exposed to $\sim 2500{ }^{\circ} \mathrm{C}$ plasma flow for $60 \mathrm{~s}$.

- Effect of GNP addition on $\mathrm{TaC}$ oxidation behavior retention and enhancement Effect of GNP investigated through microstructural characterization of formed oxide scales

\subsection{Improved Mechanical Properties}

Tantalum Carbide is an ultra high temperature ceramic (UHTC) with high Young's Modulus (435 GPa) [21], high hardness (13.5-20 GPa) [22], high melting temperature $\left(3880{ }^{\circ} \mathrm{C}\right)[23]$, and high resistivity to oxidation and chemical attack which makes it an ideal candidate for high temperature resistant aerospace applications such as scramjet engine nozzles, hypersonic aircraft, and planetary entry vehicles. However, low fracture toughness (4-5 $\mathrm{MPa} \mathrm{m}^{1 / 2}$ ) [24] of $\mathrm{TaC}$ is a limiting factor for such applications. There is a strong desire to improve the fracture toughness of all ceramics including $\mathrm{TaC}$, which has added significance because of its high temperature resistivity. Graphene has been found to have very impressive mechanical properties that make it ideal for use as a reinforcing second phase in a composite. For example, graphene has a very high tensile strength (130 GPa) [25] and a very high Young's Modulus (0.5 - 1 TPa) [26]. Graphene Nano Platelets (GNP) consist of approximately 20 - 30 graphene sheets and have a 
thickness of $5-10 \mathrm{~nm}$. [27]. GNPs are much cheaper than single layer graphene but still exhibit many of graphene's unique properties.

One of the challenges of UHTC materials is their consolidation into bulk structures. Conventional furnaces are not a plausible option as the temperatures required to melt UHTC materials exceeds the capability of most furnaces. Various sintering methods have been used to consolidate UHTC, however consolidation of $\mathrm{TaC}$ to high density with a fine grain structure has proven challenging. This challenge is addressed in this study through the use of Spark Plasma Sintering (SPS). The rapid sintering times of SPS processing have been shown to minimize grain growth in ceramics [28].

\subsection{Retention of UHTC Oxidation Behavior}

UHTC materials are expected to operate in chemically active, nonequilibrium environments and thus it is desirable to improve their oxidation behavior as much as possible. Addition of GNP is expected to improve the oxidation behavior of $\mathrm{TaC}$. Thermodynamic relations show that oxidation products of $\mathrm{TaC}$ will revert back to $\mathrm{TaC}$ upon reacting with carbon [29]. Therefore GNP and other carbon allotropes have the potential to convert tantalum oxides back to TaC. The source of carbon, in our case GNO, must survive long enough in order to react with the oxides that are formed. Oxidation behavior of TaC-GNP composites will be studied using a high temperature plasma flow in order to access whether addition of GNP has had an adverse effects on oxidation resistance. A plasma flow has a very high temperature and velocity making it more 
representative of the aerothermal environment experienced during hypersonic flight. Evaluating oxidation behavior at these conditions is preferred over conventional furnace testing.

In the following chapter, Chapter 2, a review of the state of the art is presented. Current state of UHTC materials with a focus on the properties obtained and the processing techniques used for consolidation. A review of graphene is presented as Graphene NanoPlatelets are used to reinforced $\mathrm{TaC}$ in this study. The reinforcement of composites with graphene and other carbon allotropes is discussed. Two other critical features of this study are the use of spark plasma sintering and aerothermal oxidation testing; overviews of both are presented and examined. Chapter 3 details the materials and experimental procedures used in the synthesis and evaluation of TaC-GNP composites. Chapter 4 presents the results of these experiments and an analysis of the underlying mechanisms governing the observed phenomena. Chapter 5 presents the conclusion of this research and suggests future directions for further research on TaCGNP composites. 


\section{CHAPTER II: REVIEW OF THE STATE OF THE ART}

\section{Ultra High Temperature Ceramics (UHTC)}

Tantalum Carbide $(\mathrm{TaC})$ is part of a class of materials known as Ultra High Temperature Ceramics (UHTC). UHTCs are typically carbides, nitrides, and borides of the Group IV and V transition metals. As the name implies, UHTC materials have very high melting points and other properties that make them excellent candidates for aerospace applications. In this section both the properties that make UHTC so desirable as well as those that hinder their current implementation are discussed. The challenge of processing and consolidating these materials into bulk structures that can serve as high performance engineering materials is also of considerable importance.

\subsection{Properties}

UHTC materials have many excellent properties and these properties are a function of micro and atomic scale characteristics. The chemical bonds of UHTC materials typically have characteristics of both ionic and covalent bonding. UHTC materials also have stiff crystal lattices with low lattice constants. Two of the most widely studied UHTCs are Zirconium Diboride $\left(\mathrm{ZrB}_{2}\right)$ and Hafnium Diboride $\left(\mathrm{HfB}_{2}\right)$. These materials consist of Boron atoms bonded with a Group IV transition metal. Borides have the characteristic having an increased melting temperature, hardness, strength and chemical stability as the ratio of boron atoms to metal atoms increases. These properties increase because the strength of Boron-Boron bonds increases and the crystal lattice becomes stiffer. $\mathrm{ZrB}_{2}$ and $\mathrm{HfB}_{2}$ like other UHTCs have ionic and covalent bonds, 
however, the specific interactions and configurations cause variations in properties as compared to their respective carbides or nitrides. The ionic nature of the bonds is a result of electron donor and acceptor interactions, however, the nature of covalent bonding can be more involved. Borides tend to form $\mathrm{sp}^{2}$ and $\mathrm{sp}^{3}$ hybrid bonds leading to the covalent nature of the bonds. While borides combine $\mathrm{sp}^{3}$ configurations with $\mathrm{sp}^{2}, \mathrm{~s}^{2} \mathrm{p}$, and $\mathrm{sp}$ configurations, carbides only have $\mathrm{sp}^{3}$ bonding which leads to differential properties [30]. The $\mathrm{sp}^{3}$ configuration has the highest strength and thus carbides tend to have higher melting points, hardness and strength than borides. $\mathrm{HfB}_{2}$ and $\mathrm{ZrB}_{2}$ however have higher thermal conductivities than $\mathrm{HfC}$ and $\mathrm{ZrC}[2]$.

UHTC material properties also depend on the properties of the metal atom and its interaction with $\mathrm{B}, \mathrm{C}$, or $\mathrm{N}$ atoms. In the case of diborides, $\mathrm{ZrB}_{2}$ has the largest metal atoms radius and this causes the Boron-Boron bonds to stretch leading to higher crystal lattice constants $(a$ and $c$ ). Hafnium has a smaller atomic radius than Zirconium and this is reflected in the lattice constants of these two borides. $\mathrm{ZrB}_{2}$ has a lattice constants $(a=3.17, c=3.53)$ that are slightly larger than those of $\operatorname{HfB}_{2}(a=3.14, c=3.47)$ [30]. This trend of increasing lattice parameter with increasing metal atom size is consistent in other diborides such as $\mathrm{TaB}_{2}, \mathrm{NbB}_{2}, \mathrm{TiB}_{2}$, and $\mathrm{CrB}_{2}$. Lattice constants are dependent on bond strengths and these bond strengths are responsible for several bulk properties such as hardness, bulk modulus, melting temperature, coefficient of thermal expansion, thermal conductivity, and elastic modulus. Different bond types such as boron-boron, metalmetal, and boron-metal may be more dominant in some properties than others. In borides for examples, hardness is dominated by Boron-Boron bonds and melting temperature is 
dominated by Boron-Metal bonds [31]. Borides of the largest atomic radii metals were shown to have the highest melting points while having the largest Boron-Boron lattice parameters and the weakest bond strengths. Melting temperature is dependent on the cohesive energy of bonds and these large radii metal borides had the strongest BoronMetal bonds which showed that the Boron-Metal bonding was the dominant factor in determining the melting points of transition metal borides.

In addition to high temperature resistance, the most attractive attribute of UHTCs is their high mechanical strength. UHTC tend to have high Elastic Moduli, hardness, and tensile strength. However, they are not currently suitable for industrial use because of their low fracture toughness. UHTCs have varying mechanical properties due to both atomic level properties such as those discussed above and also due to varying microstructures. One UHTC material whose fracture toughness has been improved by modification of the microstructure is $\mathrm{Ta}_{2} \mathrm{C} . \mathrm{Ta}_{2} \mathrm{C}$ grain sizes and shapes are dependent on processing temperatures and methods which will be discussed in detail in the subsequent section. $\mathrm{Ta}_{2} \mathrm{C}$ processed at $1700{ }^{\circ} \mathrm{C}$ shows elongated grains which leads to some anisotropic behavior but more importantly it provides a self-toughening effect [32]. $\mathrm{Ta}_{2} \mathrm{C}$ with elongated grains had a flexure strength $(548 \mathrm{MPa})$ that was higher than a $\mathrm{Ta}_{2} \mathrm{C}$ sample (507 MPa) with comparable densification and porosity levels but equiaxed grains. The elongated $\mathrm{Ta}_{2} \mathrm{C}$ had comparable values of fracture toughness, elastic modulus, and hardness as the equiaxed $\mathrm{Ta}_{2} \mathrm{C}$. While this elongated $\mathrm{Ta}_{2} \mathrm{C}$ microstructure provides a good combination of strength and toughness, the instability of the microstructure makes it undesirable for high temperature applications. 
The conventional approach to improving the microstructure of UHTCs is to reduce grain size and increase densification. Grain size reduction is challenging because grain growth is very common during consolidation due to the high processing temperatures. One approach to decreasing grain sizes has been to consolidate UHTCs from very fine grained starting powders. Nano sized $\mathrm{HfB}_{2}$ powders with particles sizes of $100-200 \mathrm{~nm}$ have been synthesized [33] however the results of the consolidation of these powders has yet to be reported. $\mathrm{ZrB}_{2}$ powders with particle sizes ranging from 10 $\mathrm{nm}$ to $500 \mathrm{~nm}$ were prepared and then consolidated into bulk form by Zamora et al [34]. Early results indicate formation of fine microstructures but mechanical properties have yet to be reported.

Another approach to improving mechanical properties is the addition of secondary phases which can serve as sintering aids and/or as a reinforcing phase. The addition of $\mathrm{SiC}$ in various morphologies and concentrations has shown to improve UHTC properties [35]. Silicon carbide particles have been shown to improve hardness and strength of $\mathrm{ZrB}_{2}$ however toughness often remains unchanged. The difference in the coefficient of thermal expansion (CTE) between $\mathrm{SiC}$ and $\mathrm{ZrB}_{2}$ has been shown to generate residual stresses [36]. The size of the starting $\mathrm{ZrB}_{2}$ and $\mathrm{SiC}$ powders has also been shown to have an effect on properties. Finer starting powders of $\mathrm{ZrB}_{2}$ and $\mathrm{SiC}(2 \mu \mathrm{m}$ and $.5 \mu \mathrm{m}$ respectively) were shown to have lower flexure strengths at $1800{ }^{\circ} \mathrm{C}(112 \mathrm{MPa}$ vs $217 \mathrm{MPa})$ than composites where the starting powders were $5 \mu \mathrm{m} \mathrm{ZrB}_{2}$ and $2 \mu \mathrm{m} \mathrm{SiC} \mathrm{[37].} \mathrm{Elongated}$ reinforcements such as $\mathrm{SiC}$ whiskers [38, 39], SiC chopped fibers [38-41], and carbon fibers [42] has proven effective in increasing toughness in $\mathrm{ZrB}_{2}$ composites. Sciti et al 
[40] investigate various compositions of $\mathrm{SiC}$ chopped fibers reinforced $\mathrm{ZrB}_{2}$ composites and find that toughness increases with volume percent (vol.\%) SiC fibers until 20 vol. \% and begins to decrease at 30 vol. $\%$. The sample with 20 vol. $\% \mathrm{SiC}$ chopped fibers has a fracture toughness of 5.3 - 5.6 MPa m ${ }^{0.5}$ while the pure $\mathrm{ZrB}_{2}$ had a fracture toughness of 3.7 MPa $\mathrm{m}^{0.5}$. These samples however displayed a decrease in flexure strength with increasing $\mathrm{SiC}$ chopped fiber content. The pure $\mathrm{ZrB}_{2}$ has a flexural strength of $600 \mathrm{MPa}$, this decreased to $400 \mathrm{MPa}$ with $10-20$ vol. $\% \mathrm{SiC}$ chopped fibers and down to $200 \mathrm{MPa}$ with 30 vol. \% chopped fibers. $\mathrm{ZrB}_{2}$ reinforced with $\mathrm{SiC}$ whiskers showed less of an improvement in fracture toughness but also experienced a lower loss of flexural strength [40].

Another reinforcing phase for UHTCs that has been explored is tungsten carbide (WC). WC was added to a system of $\mathrm{HfB}_{2}-\mathrm{SiC}$ system (20 vol.\% $\left.\mathrm{SiC}\right)$ and it was found that an addition of 5 and 10 weight percent (wt. \%) WC improved the oxidation resistance, the elastic modulus, and the flexure strength $[43,44]$. The addition of $10 \mathrm{wt}$. $\% \mathrm{WC}$ led to a higher increase in densification, hardness, fracture toughness, and flexural strength as compared with the addition of 5 vol.\% WC. The grain size was also lower in the composite with 10 wt. \% WC and the Young's moduli were comparable. Like SiC, WC is a refractory ceramic and it has been shown to improve the mechanical properties and oxidation resistance in $\mathrm{ZrB}_{2}$ matrices as well. The addition of other ceramic sintering additives such as $\mathrm{B}_{4} \mathrm{C}$ has also proven effective in enhancing densification and properties of $\mathrm{HfB}_{2}[45]$. 
One of the reasons the $\mathrm{ZrB}_{2}$ and $\mathrm{HfB}_{2}$ are two of the most studies UHTCs is the fact that they have good solid solubility with other transition metal borides which allows for some optimization of properties. Solid solubility is largely determined by atomic radii and there is a less than $10 \%$ difference in the atomic radii of $\mathrm{Zr}, \mathrm{Hf}, \mathrm{Ti}$, Ta, and $\mathrm{Nb}$ [30]. Similar atomic radii lead to low energies of formation and thus higher solid solubility. The activation energies of transition metal borides is similar to the activation energy for metal atom diffusion in these borides indicating that diffusion of metal atoms is the mechanism for formation of transition metal boride solid solutions.

Tantalum Carbide $(\mathrm{TaC})$ is one of the UHTCs that has been less widely reported on. TaC has the highest melting point of all UHTC materials $\left(\sim 3880{ }^{\circ} \mathrm{C}\right)[23]$ and this makes consolidation into bulk structures even more challenging than for other UHTCs. While consolidation of $\mathrm{TaC}$ is more challenging, the benefits of doing so are worthwhile as $\mathrm{TaC}$ has been reported to have higher oxidation resistance and mechanical properties than other UHTC materials [46]. The stability of $\mathrm{TaC}$ at high temperatures $\left(>3700{ }^{\circ} \mathrm{C}\right)$ can be seen from its phase diagram shown below in Fig. 2.1. TaC can have nonstoichiometric compositions which are stable at high temperatures as can be seen from the phase diagram. The flexural strength of $\mathrm{TaC}$ has been reported at around $400-700$ $\mathrm{MPa}[22,47,48]$. This is higher than other transition metal carbides such as $\mathrm{ZrC}$ which has flexural strengths around $200-400 \mathrm{MPa}[49,50]$. As with other UHTCs fracture toughness is low (4-5 $\mathrm{MPa} \mathrm{m}^{0.5}$ ) [24], so secondary phases have been added in order to serve as reinforcement as well as a sintering aid. $\mathrm{SiC}$ has shown promising results as a secondary phase for $\mathrm{TaC}$ as with the more widely studied $\mathrm{ZrB}_{2}$ and $\mathrm{HfB}_{2}$. The addition of 


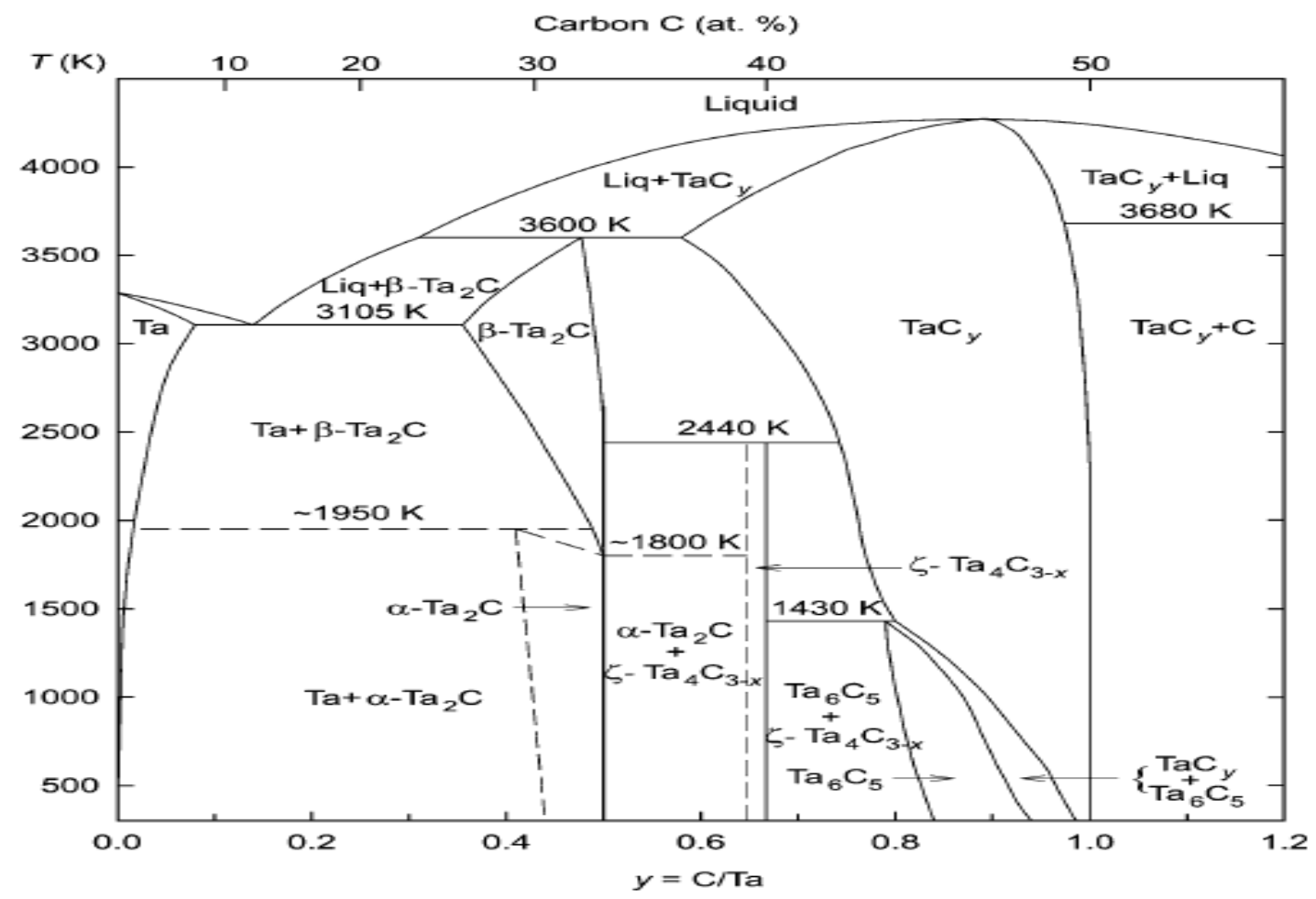

5 vol. $\% \mathrm{SiC}$ yielded $97.5 \%$ densification [51] while the addition of 20 vol.\% $\mathrm{SiC}$ led to densification above $98 \%$ [46]. The addition of $20-40$ vol. $\% \mathrm{SiC}$ has been shown to not only improve mechanical properties in UHTCs but also the oxidation resistance. The addition of 20 vol. \% SiC improved the flexural strength from $500 \mathrm{MPa}$ to $599-715$ MPa depending on the sintering temperature. The TaC-SiC composites had elastic moduli between $511 \mathrm{GPa}$ and $537 \mathrm{GPa}$ and hardness values ranging from19.2 GPa to $20.3 \mathrm{GPa}$. Reported values of elastic moduli and hardness values of pure $\mathrm{TaC}$ have had a relatively large scatter but most tend to be around $435 \mathrm{GPa}$ [21] and $16.8 \mathrm{GPa}$ respectively [22]. The fracture toughness of the 20 vol. $\% \mathrm{SiC}$ reinforced $\mathrm{TaC}$ improved to $6.7 \mathrm{MPa} \mathrm{m}^{0.5}$ as compared with the pure $\mathrm{TaC}$ which had a fracture toughness of $3.4 \mathrm{MPa} \mathrm{m}^{0.5}$. 


\subsection{Processing}

The processing of UHTC materials is perhaps the most important aspect of UHTC research as the processing determines the materials microstructure and thus its properties. UHTCs cannot be consolidated to high densification using conventional furnaces as the operating temperatures are usually no higher than $2000{ }^{\circ} \mathrm{C}$. UHTCs have melting points much higher than $2000{ }^{\circ} \mathrm{C}$ and thus higher temperatures would be required to melt the material and form it into a useable shape. UHTCs such as TaC which have melting points approaching $4000{ }^{\circ} \mathrm{C}$ are the most challenging to consolidate. UHTCs are consolidated by sintering techniques that combine a high pressure and high temperature cycle in order to overcome the strong covalent bonds and low diffusion constants. UHTCs have also been consolidated into bulk in the form of coatings by methods such as vacuum plasma spraying [52] and chemical vapor infiltration [53]. UHTC coating synthesis will not be elaborated on in this thesis as bulk structures are the focus. The variations in sintering techniques vary and each has its advantages and disadvantages. It is not desirable however for the sintering cycle to be excessively long as prolonged exposure to high temperatures will lead to grain growth and result in weaker mechanical properties. In an effort to minimize grain growth the processing of the starting powder has also received significant attention. Smaller starting particle sizes lead to smaller grains and both physical and chemical methods have been utilized to decrease starting powder particle size. The use of different sintering aids can also be used to enhance densification or other material properties. 
The first step in processing a UHTC is usually to start with powder and synthesizing UHTC powder has been accomplished with several techniques. The first class of techniques consists of reduction processes. For UHTC diborides, one usually begins with metal oxides and then reduces them through carbothermal reactions, borothermal reactions, or a combination of these reactions. Examples of carbothermal, borothermal, boron carbide, and combined reactions are shown below in Eqns. (2.1)-(2.4) [30] :

Carbothermal Rxn: $Z_{2} \mathrm{O}_{2(s)}+\mathrm{B}_{2} \mathrm{O}_{3_{(l)}}+5 \mathrm{C}_{(g)} \rightarrow \mathrm{ZrB}_{2(s)}+5 \mathrm{CO}_{(g)}$

Borothermal Rxn: $\quad \mathrm{ZrO}_{2(s)}+3 B_{(s)} \rightarrow \mathrm{ZrB}_{2(s)}+\mathrm{B}_{2} \mathrm{O}_{3(g)}$

Boron Carbide Rxn: $7 \mathrm{ZrO}_{2}(s)+5 B_{4} C_{(s)} \rightarrow 7 \mathrm{ZrB}_{2(s)}+3 \mathrm{~B}_{2} \mathrm{O}_{3(g)}+5 \mathrm{CO}_{(g)}$

Combined Rxn: $\quad 2 \mathrm{ZrO}_{2(s)}+B_{4} C_{(s)}+3 C_{(g)} \rightarrow 2 \mathrm{ZrB}_{2(s)}+4 \mathrm{CO}_{(g)}$

These same reactions could be used to synthesize $\mathrm{HfB}_{2}$ powders; one would just replace $\mathrm{Zr}$ in the equations above with $\mathrm{Hf}$. The completion of these reactions requires temperature above $2000{ }^{\circ} \mathrm{C}$ as well as an efficient mode of removing the large amount of gases produced. Common impurities in these powders include trace amounts of carbons and carbides.

Another class of techniques and perhaps the simplest is reactive processes where the metal is reacted with boron/carbon resulting in borides or carbides. Challenges here include having a high purity source of Boron as well as operating in an inert environment to prevent formation of oxides or other undesired products. These reactions are also highly exothermic and temperatures can exceed melting points of $\mathrm{Zr}$ and $\mathrm{Hf}$ leading to 
local melting which is the basis for self-propagating high-temperature synthesis (SHS). Powders produced by SHS have high defect concentrations which aid in the sinterability of the powders [54].

Another method of producing UHTC powders is through chemical routes. These methods are more complex but have produced powders with particle sizes as low as 10$20 \mathrm{~nm}$. The reaction of $\mathrm{HfCl}_{4}$ with $\mathrm{NaBH}_{4}$ is one such reaction, it is shown below in Eqn. (2.5) [55]:

$$
\mathrm{HfCl}_{4(g)}+\mathrm{NaBH}_{4(g)} \rightarrow \mathrm{HfB}_{2(s)}+2 \mathrm{NaCl}_{(s)}+2 \mathrm{HCl}_{(g)}+3 \mathrm{H}_{2(g)}
$$

Chemical routes have also been use to synthesize other UHTCs such as TaC. One such method reacts Tantalum Pentachloride $\left(\mathrm{TaCl}_{5}\right)$ magnesium powder which forms tantalum metal and magnesium chloride [56]:

$$
\mathrm{TaCl}_{5}+\mathrm{Mg} \rightarrow \mathrm{Ta}+\mathrm{MgCl}_{2}
$$

This reaction is highly exothermic and the high heats generated enable the following reaction to occur simultaneously:

$$
\mathrm{Na}_{2} \mathrm{CO}_{3}+\mathrm{Heat} \rightarrow \mathrm{CO}_{2}+\mathrm{Na}_{2} \mathrm{O}
$$

The carbon dioxide produced from the reaction above reacts with the magnesium powder in the LHS of Eqn. (2.6) and produces carbon as follows:

$$
\mathrm{CO}_{2}+\mathrm{Mg} \rightarrow \mathrm{C}+\mathrm{MgO}_{2}
$$


The carbon then reacts with the tantalum metal that was formed during the initial reaction (Eqn.2.6), and Tantalum Carbide is formed. The overall reaction of the above processes is given by Eqn. (2.9) below:

$$
2 \mathrm{TaCl}_{5}+2 \mathrm{Na}_{2} \mathrm{CO}_{3}+9 \mathrm{Mg} \rightarrow 2 \mathrm{TaC}+5 \mathrm{MgCl}_{2}+4 \mathrm{MgO}+2 \mathrm{Na}_{2} \mathrm{O}
$$

Synthesized UHTC powders are widely available commercially and most researchers obtain them commercially rather than synthesizing it themselves. This synthesized powder is usually processed further before sintering by high energy ball milling, also referred to as attrition milling. This process consists of powder being placed in a jar which may be made of a metal, ceramic, or polymer depending on the application and setup. Ceramic or metal balls are placed inside the jar as well, this is referred to as the milling media. The jar is shut and then rotated in an oscillating motion causing the motion of the balls and powder to not be purely centripetal. The balls are ideally harder than the powder as the main purpose is to crush the powder into smaller particles. For UHTC materials a hard ceramic such as silicon nitride, alumina, or tungsten carbide is used. Ceramics are brittle and some chipping of the balls occurs. Impurities from the balls lead to trace amounts of $\mathrm{WC}, \mathrm{Al}_{2} \mathrm{O}_{3}, \mathrm{Si}_{3} \mathrm{~N}_{4}$, etc being found in the ball milled powders [30]. The rapid cycling of the jar leads to high temperatures and pressures being developed in the jar. Phase analysis should be done, especially if material being ball milled can react with the jar. Sometimes a slurry is made inside the jar by adding acetone, ethanol, or other liquid media in order to provide some cooling and better dispersion of powders that are being mixed. 
Ball milled powders have been shown to sinter better mostly because of the decrease in particle size. Ball milling not only increases surface area but it may also increase surface defects which also enhances sintering. Zamora et al $[34,57]$ show that ball milling of powders to the nanoscale level leads to a substantial increase in densification at low sintering temperatures. It is shown that the temperature needed to sinter $\mathrm{ZrB}_{2}$ by SPS decreases as the powder particle size decreases. Small particle sizes combined with low sintering temperatures lead to small grain sizes while still attaining high densification. $10 \mathrm{~nm} \mathrm{ZrB}_{2}$ powder sintered at $1625{ }^{\circ} \mathrm{C}$ reached a densification of $98.5 \%$. Zamora et al [34] propose that the nanoscale refinement is necessary as only it provides significant increases in grain boundary diffusion at lower temperatures. Grain boundary diffusion is the primary mechanism in the sintering and densification of UHTC materials.

Hot pressing (HP) has been reported to be one of the most effective techniques for obtaining high density UHTCs $[22,44,47,58,59]$. $\mathrm{Ta}_{2} \mathrm{C}$ has been consolidated from $\mathrm{Ta}$ and $\mathrm{TaC}$ powders mixtures by $\mathrm{HP}$ at $1630^{\circ} \mathrm{C}$ and $195 \mathrm{MPa}$ for 2 hours [60]. Hot pressed $\mathrm{ZrB}_{2}$ reinforced with 20 vol.\% $\mathrm{SiC}$ has led to $100 \%$ densification and $\mathrm{ZrB}_{2}$ reinforced with 14 vol.\% $\mathrm{SiC}$ and 30 vol. \% carbon led to $99.9 \%$ densification [61]. HP parameters used to consolidate borides typically consist of a temperature above $2000{ }^{\circ} \mathrm{C}$ with a pressure of 20-30 MPa [30]. HP can also be done using lower temperatures of 1790 $1840{ }^{\circ} \mathrm{C}$ however very large pressures in the range of $800-1500 \mathrm{MPa}$ are needed [30]. Secondary phases which form silica $(\mathrm{SiO} 2)$ have been found to improve oxidation resistance of hot pressed UHTCs. As mentioned before, $\mathrm{SiC}$ has been widely studied 
however $\mathrm{MoSi}_{2}$ and $\mathrm{TaSi}_{2}$ reinforced UHTCs consolidated by hot pressing have also been studied and shown to improve oxidation resistance [62- 64]. The use of silica formers leads to a protective glassy phase that inhibits the infiltration of oxygen during oxidation.

Another sintering technique is pressure less sintering and this method has shown much promise in consolidating near net shape UHTCs [30, 65]. Pressure less sintering has been shown to consolidate $\mathrm{TaC}$ to $97.5 \%$ at $2300 \mathrm{C}$ [66]. This level of densification is comparable with that obtained with hot pressing. The absence of an applied pressure enables the consolidation to occur in an irregular vessel leading to more complex shapes. The application of pressure is usually done axially or isotatically which means the vessel should be axially symmetrical thus limiting the variability of vessel shape. Pressure sintering was used to obtain $99 \%$ densification of a $\mathrm{HfB}_{2}$ composite with $\mathrm{SiC}$ and $\mathrm{WC}$ as secondary phases [43]. SiC and $\mathrm{WC}$ are chosen as secondary phases over $\mathrm{MoSi}_{2}$ because of their stability at higher temperatures. $\mathrm{MoSi}_{2}$ softens at high temperatures making it undesirable for high temperature applications despite its performance as a sintering aid. $\mathrm{MoSi}_{2}$ fills grains in the matrix as it softens which fills voids and increases mass transport which results in higher densification. The $\mathrm{HfB}_{2}$-SiC-WC composites are sintered at 2000 ${ }^{\circ} \mathrm{C}, 2100{ }^{\circ} \mathrm{C}$, and $2200{ }^{\circ} \mathrm{C}$ with varying amounts of WC. Densification increases both as function of sintering temperature as well as increasing WC content. Densification substantially improved at $2100{ }^{\circ} \mathrm{C}$ as compared to $2000{ }^{\circ} \mathrm{C}$. The difference in densification at $2100{ }^{\circ} \mathrm{C}$ and $2200{ }^{\circ} \mathrm{C}$ is minimal. The effect of WC addition levels off at around $5 \mathrm{wt} . \% \mathrm{WC}$ indicating that this is the optimum WC concentration for this system. The addition of $\mathrm{WC}$ as well as other ceramic sintering aids is believed to improve 
sintering by removing oxide impurities that are present. $\mathrm{HfO}_{2}$ is present in $\mathrm{HfB}_{2}$ powders and the following reaction (Eqn. 2.10) between $\mathrm{WC}$ and $\mathrm{HfO}_{2}$ consumes $\mathrm{HfO}_{2}$ and produces $\mathrm{HfC}$ which is a high strength UHTC with a high melting point.

$$
3 \mathrm{WC}+\mathrm{HfO}_{2} \rightarrow \mathrm{HfC}+3 \mathrm{~W}+2 \mathrm{CO}
$$

WC also reacts with $\mathrm{HfB}_{2}$ which leads to additional formation of $\mathrm{HfC}$ as well as WB. Having a sintering aid that reacts with and consumes oxide impurities allows powders with higher impurity levels to be used to obtain high strength UHTCs which decreases costs associated with obtaining high purity powders.

High frequency induction heating is a sintering technique employing a high pressure concurrently with an induced electric current. High frequency induction heating has been used to consolidate $\mathrm{TaC}$ to high densities without high levels of grain growth [24]. This process is promising because of its rapid sintering times which limit grain growth. The $\mathrm{TaC}$ reached $96 \%$ densification by sintering under a $80 \mathrm{MPa}$ pressure and an induced current for 3 minutes. Densification and hardness increased with increasing ball milling time as expected. Hardness value obtained for strongest sample was $22 \mathrm{GPa}$, this is a very high value and is likely a product of both the small starting powder size due to increased milling time as well as the rapid sintering of this process.

One of the most promising sintering methods is spark plasma sintering (SPS). This method combines an axial pressure, high temperature, and a pulsing current. SPS has very high heating and cooling rates which minimizes grain growth while still leading to high density compacts. Several studies have shown SPS having great potential for 
efficient consolidation of UHTCs [32, 46, 67-69]. Mechanisms, advantages, and disadvantages of SPS will be discussed in detail in a later section of this thesis as its importance merits a more in depth discussion.

Some of the UHTCs that have been consolidated by SPS will be discussed here along with the effect of processing parameters on microstructures. $\mathrm{Ta}_{2} \mathrm{C}$ was consolidated by SPS at $1300{ }^{\circ} \mathrm{C}, 1700{ }^{\circ} \mathrm{C}$, and $1900{ }^{\circ} \mathrm{C}$. Densification occurred at $1300{ }^{\circ} \mathrm{C}$ which is much lower than the temperatures required during hot pressing. The microstructure was different at each processing temperature with the compact sintered at $1700{ }^{\circ} \mathrm{C}$ displaying an elongated grain structure [33]. The variation of the $\mathrm{Ta}_{2} \mathrm{C}$ microstructure with relatively low changes in processing condition makes $\mathrm{Ta}_{2} \mathrm{C}$ undesirable for high temperature applications where large temperature gradients are experienced [2?].

SPS is used to consolidate a TaC-SiC composite at sintering temperatures of 1600 ${ }^{\circ} \mathrm{C}, 1700{ }^{\circ} \mathrm{C}, 1800{ }^{\circ} \mathrm{C}$, and $1900{ }^{\circ} \mathrm{C}$ [46]. All samples were held for 5 min under a $40 \mathrm{MPa}$ pressure and the densification achieved was no lower than $98 \%$. As the temperature increased so did the densification, with the sample sintered at $1900{ }^{\circ} \mathrm{C}$ reaching $100 \%$ densification. While the grain size increased slightly with increasing temperature this did not have an adverse effect on properties. Elastic modulus, hardness, fracture toughness, and flexure strength all increased with increasing temperature and the sample sintered at $1900{ }^{\circ} \mathrm{C}$ had the highest properties. The SiC particles became elongated at $1900{ }^{\circ} \mathrm{C}$ which likely contributed to the improvement in properties especially the fracture toughness.

The effect of different pressures during SPS processing of UHTCs has also been investigated. TaC along with TaC-B4C and TaC-CNT composites are sintered at $1850{ }^{\circ} \mathrm{C}$ 
and pressures of $100 \mathrm{MPa}, 255 \mathrm{MPa}$, and $363 \mathrm{MPa}[68,69]$. Densification is shown to increase with pressure for all compositions. The increase in densification is most pronounced for the pure $\mathrm{TaC}$ sample while the samples with $\mathrm{B}_{4} \mathrm{C}$ and $\mathrm{CNT}$ as sintering aids exhibit smaller increases in densification with the increasing pressure. The increased pressure causes an increase in grain size in the pure $\mathrm{TaC}$ sample. Bakshi et al [68] attribute this to higher heat transfer and increased movement of interfaces as a result of the higher pressures. The samples reinforced with $\mathrm{B}_{4} \mathrm{C}$ and $\mathrm{CNT}$ do not exhibit increased grain growth at higher pressures as $\mathrm{B}_{4} \mathrm{C}$ and $\mathrm{CNT}$ act as grain growth inhibitors during sintering. Despite the larger grains in the pure $\mathrm{TaC}$ samples the hardness and elastic

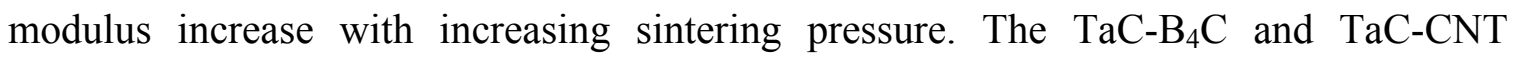
samples also exhibit higher hardness with increasing processing pressure.

While current processing technology only allows for relatively small bulk samples of UHTCs to be produced this is not a critical issue for most UHTC applications. UHTCs will be largely employed for leading edges of hypersonic vehicles and typically these components do not need to be very large. The stagnation point of the hypersonic aircraft is the leading edge and this is where the highest temperatures are experienced. However, the temperature quickly decreases as the distance from the leading edge increases. This is because heat is conducted away from the stagnation point and radiated back to the environment as shown in Fig. 2.2. This concept accentuates the need for UHTC materials to have a high thermal conductivity so heat may be efficiently transferred away from the leading edge and then rejected back to the environment through radiation. If the thermal conductivity is very low then heat transfer will be a very slow process and the leading 


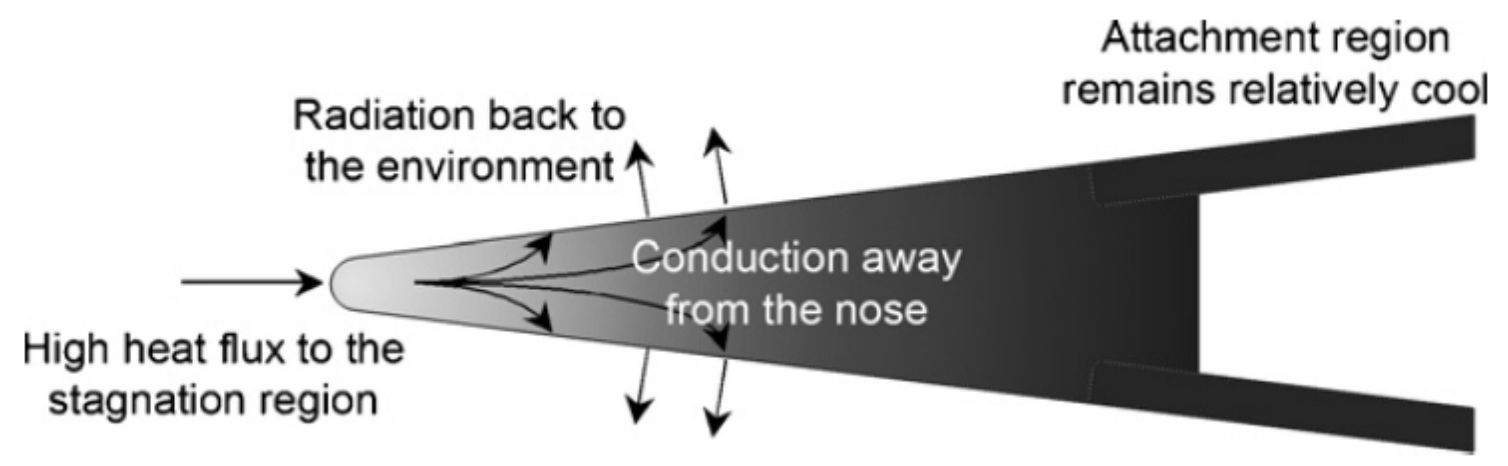

edge will heat up at a very high rate. The small size of the components needed for UHTC leading edges also means that the ensuing weight penalty of using dense materials such as $\mathrm{TaC}$ is not a concern. Instead, the use of $\mathrm{TaC}$ or other high density UHTCs can be favorable to maintaining aerodynamics stability by shifting the center of gravity toward the front of the vehicle and ahead of the aerodynamic center.

Another challenge is processing of UHTC materials is sufficient process control. In order for these materials to be considered an industrially viable option they must be able to exhibit consistent properties under given processing parameters. Currently large data scatter is common for mechanical properties within a given sample. For example, flexural strengths of a $\mathrm{ZrB}_{2}-\mathrm{SiC}$ composite had a range which was over $50 \%$ of the mean value. Such variability indicates that even in a given sample the variability in properties and structure can be large. The variability from batch to batch would likely have equal or greater variability. Processing techniques need to strive towards not only property enhancement but also reproducibility of microstructures and properties. 


\section{Graphene}

Graphene is a 2-D allotrope of carbon consisting of a sheet of $\mathrm{sp}^{2}$ bonded carbon atoms. The molecular model of graphene shown in Fig. 2.3a shows the honeycomblattice structure of graphene, while the TEM micrograph in Fig. 2.3b shows the thickness of this atom-thick structure. Single layer graphene can be difficult and expensive to isolate and thus multi-layer graphene such as Graphene Nano Platelets (GNP) are often used as they retain many of the desirable mechanical properties of single layer graphene. Graphene Nano Platelets consist of approximately 20 - 30 graphene sheets and have a thickness of $5-10 \mathrm{~nm}$. [27]. The sheets of graphene in GNPs are held together by van der waals forces and have been experimentally measured to have a spring constant of 1 $5 \mathrm{~N} / \mathrm{m}$ [26]. GNP, like graphene, have a very high surface area and interfacial energy
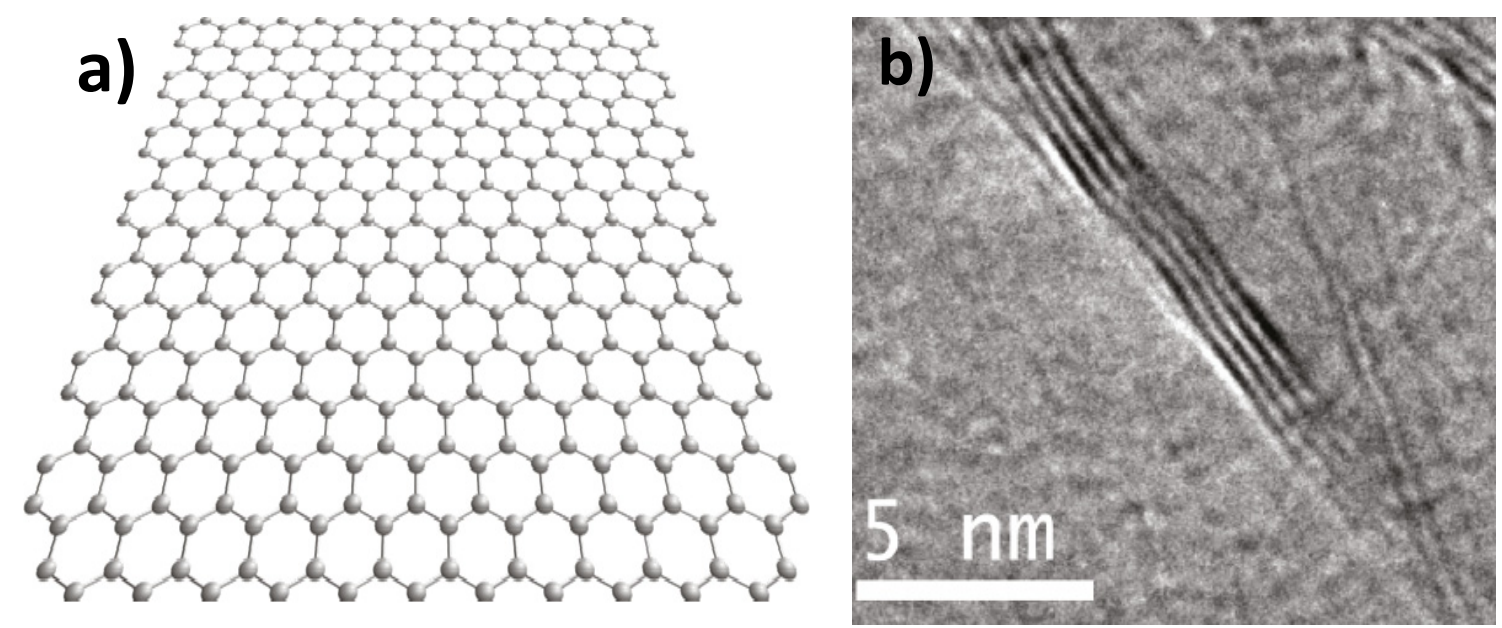

which results in strong interfacial bonding in composite materials. Graphene has been found to have very impressive mechanical properties that make it ideal for use as a 
reinforcing second phase in a composite. For example, graphene has a very high tensile strength (130 GPa) [25] and a very high Young's Modulus (0.5 - $1 \mathrm{TPa})$ [26].

Graphene was known to exist for several decades before its first isolation in 2004, however its synthesis was not thought to be feasible. Many scientists believed that graphene was an unstable allotrope and that even if a single graphene sheet could be isolated it would then roll up into a structure resembling a carbon nanotube (CNT). Graphene sheets as thin as $5 \mathrm{~nm}$ were actually isolated in 1960 [71] however little attention followed on the graphene front. Instead the methods used to thin graphite down to such a layers of graphene ( $\sim 15$ layers) became widely applied for preparing samples for electron microscopy. The means of isolation in 2004 was also a mechanical exfoliation method, however such methods are not typically suitable for large production. The primary methods of producing graphene are mechanical exfoliation, epitaxial growth, graphene oxide reduction, and molecular approaches.

The basis of mechanical exfoliation arises from the fact that graphene layers are held together by weak van der waals forces. The van der waals interaction energy between graphene layers is $2 \mathrm{eV} / \mathrm{nm}$ which translates to a force of $300 \mathrm{nN} / \mu \mathrm{m}^{2}$ being needed to pull or exfoliate a layer of graphene [72]. This force can easily be attained using adhesive tape and this is the method used to first isolate graphene in 2004. The tape was adhered and peeled of several times and when an atomically thick layer was obtained a heat treatment was applied to rid the surface of any glue residues from the tape. This method has also been modified by employing different substrates and controlling the bonding between the graphite and the substrate. Mechanical exfoliation techniques have 
been able to extract $\sim 10$ micron graphene flakes [73] indicating that the method has some promise for large scale production.

Epitaxial growth of graphene by chemical vapor deposition (CVD) on metal or metal carbide substrates holds great promise for electronics applications. Of particular interest is the growth of graphene on $\mathrm{SiC}$ wafers by decomposing $\mathrm{SiC}$ so that $\mathrm{Si}$ atoms sublimate and the remaining carbon atoms become activated by the high temperature $\left(1300{ }^{\circ} \mathrm{C}\right)$ employed and undergo graphitization $[74,75]$. Graphene has also been grown on metal substrates by decomposition of hydrocarbons, similar to the way carbon nanotubes are grown. Sheets of graphene $100 \mu \mathrm{m}$ in length were grown by decomposing ethylene at $1420 \mathrm{~K}$ onto an Ru substrate. Graphene islands nucleate and grow on Ru by lowering the temperature to $1100 \mathrm{~K}$ which causes supersaturation. The first graphene layer that appears has a strong interaction with the metal substrate and shields the following layers from any significant interaction with the substrate. The strong interaction between the metal and the first layer of graphene contributes to an ordered high quality graphene structure but this same interaction also hinders lateral extension of the graphene thus limiting the size of graphene sheets. A balance between lateral growth and graphene structure is needed and Iridium substrates have shown to provide a combination of high quality growth along with a weaker interaction with the substrate allowing for larger sheets to be made. Weak interactions between graphene and substrate also allow for the graphene sheets to be easily transferred onto other surfaces. Other variations of CVD epitaxial growth includes plasma enhanced CVD (PECVD) and microwave PECVD (MW-PECVD). PECVD has enabled graphene to be grown a wide 
variety of substrates including transition metals, stainless steels, and oxides. The electric field due to the plasma leads to graphene growth occurring vertically during PECVD. MW-PECVD also grow vertically and one study showed a growth rate $(1.6 \mu \mathrm{m} / \mathrm{min})$ [76] which was 10 times the rate of most other CVD processes.

Graphene oxide reduction is a technique used to produce graphene by chemically breaking the bonds holding graphene layers together. The van der waals forces are weakened by inserting chemical reactants between the graphene layers which causes the graphene layers to sit on top of each other without any bonding force. The reactants form products that produce a pressure in between the graphene layers and this provides the force to complete the chemical exfoliation of a graphene layer [72]. The challenge with this route is the oxidation of the graphene layers into graphite oxide. Graphene oxide consists of both $\mathrm{sp} 2$ and $\mathrm{sp} 3$ bonding and thus its properties are significantly different than those of graphene. The reduction of graphite oxide into graphene layers has still not been fully mastered as a full $\mathrm{sp}^{2}$ structure has not been obtained. The properties of graphene produced by graphite oxide reduction however are still similar to that of fully $\mathrm{sp}^{2}$ bonded graphene and the ease of this method gives it great potential for large scale production.

There are several different types of molecular approaches to producing graphene. Among these methods one type aims to form graphene by manipulating carbon allotropes, namely nano diamond (ND) and multi-walled carbon nanotubes (MWCNT), as graphene is the building block of all carbon allotropes. ND was electro chemically deposited onto highly oriented pyrolitic graphite (HPOG) followed by a $1600{ }^{\circ} \mathrm{C}$ heat 
treatment to induce graphitization [77]. This technique successfully leads to few layer graphene (FLG) as $\mathrm{sp}^{3}$ bonds in ND are converted to $\mathrm{sp}^{2}$ bonds. Graphene can also be extracted from MWCNT by insertion of reactants in the MWCNT $[78,79]$ or by plasma etching [80]. The most successful of these techniques manage to "unzip" the CNT, that is, the cylindrical structure is cleaved resulting in graphene layers.

As alluded to earlier, single layer graphene is often not the best choice for all applications. Multi-layer graphene retains a very high surface area and has been shown the ability to be non-covalently functionalized without affecting its properties [81]. Multilayer graphene may be more favorable in some applications and thus the controlled fabrication of multi-layer graphene is desired. Multi-layer graphene synthesis is challenging as each layer should line up with the adjacent layers in such a way that stability is promoted. If there is a mismatch between layers van der waals forces may have a distribution throughout the plane which would results in distortions and ripples across the graphene sheets. As these distortions accumulate some areas might adopt a graphitic structure instead of a layered graphene structure.

Several studies have identified some variables controlling the growth of single and multi-layer graphene. Kaplas et al [82] grow graphene on ruptured copper droplets that were deposited in a fused silica substrate. The fact that the copper is ruptured allows the graphene to grow both on the surface of the copper as well as the copper-silica interface. The graphene grown at the silica interface is commensurate with the quality of that grown on the copper droplet surfaces. It is shown that the number of graphene layers grown can be controlled by adjusting the thickness of the copper droplets and the 
temperature used during the CVD process. A maximum CVD temperature of above 900 $\mathrm{C}$ is need for graphene growth and growth tends to increase with increasing temperature. The growth of graphene was also more effective as the thickness of the copper film decreased.

Wassei et al [83] also investigate growth of graphene on copper foils while varying the types of hydrocarbons and the pressure during CVD. Heavier hydrocarbons provide more carbon for the graphitization process and thus are better for producing bilayer and multilayer graphene. Heavier hydrocarbons such as ethane and propane also have weaker carbon-hydrogen bonds causing them to break apart with less energy input during CVD. Increasing the pressure leads to higher carbon concentrations which induce the formation of multiple graphene layers. The CVD of ethane at $250 \mathrm{mTorr}$ consistently formed single layer graphene while the CVD of propane at the same pressure led to bilayer graphene. Increasing the pressure to $500 \mathrm{mTorr}$ and $1000 \mathrm{mTorr}$ increased the carbon substantially and multi-layer graphene was formed.

The production of large-scale graphene is desirable and one means of accomplishing this is by joining smaller sheets of graphene. One method that has shown success in joining graphene sheets involves the use of a transmission electron microscope-scanning tunneling microscope (TEM-STM) electron beam to heat up graphene sheet edges and promote diffusion [84]. This method was able to successfully extend graphene sheets while maintaining mechanical and electrical properties. The voltage applied by the TEM-STM lead to self-assembly and rearrangement of carbon atoms at the interface between the two graphene sheet edges. This method is effective in 
extending both single layer and multi-layer graphene sheets. The application of a voltage to graphene edges can also be used to repair sections of graphene sheets that have become damaged. This method could not however be translated into the joining of graphene layers. Electrical resistance measurements show that graphene layers were merely sitting on top of each physically and not chemically joined. The layers also were separated easily indicated no chemical joining. Increasing the applied voltage did not promote joining of graphene layers either; instead graphene layers began to shrank and then evaporate as the voltage increased. The joining of multiple layers does not involved the rearranging and self-assembly of carbon networks as in the case of graphene edge joining. 


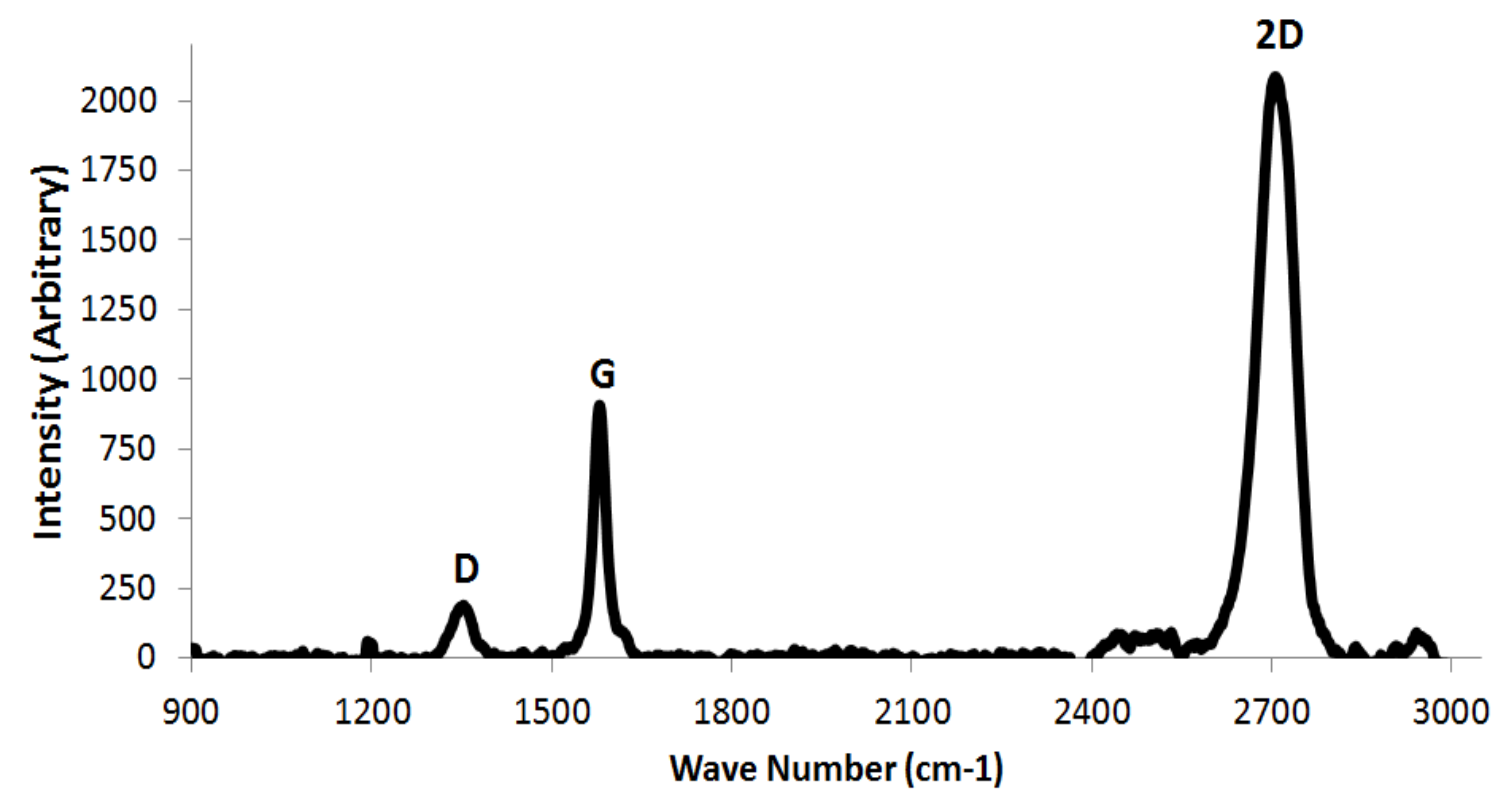

The characterization of graphene presents novel challenges because of the unique size and geometry. This thesis focuses on the use of graphene for structural composites and thus the characterization of graphene structure and mechanical properties are of utmost importance. Raman spectroscopy and atomic force microscopy (AFM) are two widely used techniques to characterize the structure and strength of graphene. Raman spectroscopy is an optical technique that gives fundamental physical information regarding the interactions of phonons and electrons in a material. The distinct raman spectra of graphene is shown in Fig. 2.4, and information of the graphene structure can be derived from the peaks in the raman spectra. The G peak $\left(\sim 1580 \mathrm{~cm}^{-1}[85-88]\right)$ is the crystallization peak of graphene and is present in all carbon materials with varying peak intensities and peak widths. The D peak $\left(\sim 1360 \mathrm{~cm}^{-1}[85-88]\right)$ becomes active in the presence of a defect and can therefore be used to assess damage in a graphene structure. The D peak is not present in high quality single layer graphene such as that produced by 
mechanical exfoliation. The 2D peak (also known as $\left.\mathrm{G}^{\prime}\right)\left(\sim 2700 \mathrm{~cm}^{-1}[85-88]\right)$ is the second order of the D peak and is sometimes referred to as the G' peak. The ratio of the intensity of the D peak divided by the intensity of the G peak gives a measure of the level of defects in a graphene structure. This important ratio can be used to evaluate the integrity of synthesized graphene or to access the damage that graphene has undergone due to exposure to some environment or processing treatment. The $2 \mathrm{D}$ peak can be used to access the number of graphene layers present in a graphene structure. The intensity ratio of $2 \mathrm{D} / \mathrm{G}$ peak is almost 4 for single layer graphene [85] and decreases with an increase in the number of layers [85-89]. The intensity of 2D band becomes lower than G for bulk graphite [85-89]. Moreover, the 2D peak in single layer graphene is a narrow and sharp one, which gets broadened and shows sign of splitting with increasing number of graphene layers [85-89]. The Raman spectra of graphene has also been shown to be affected by compression or tension of graphene enabling Raman to be used to access the stress state that the graphene has experienced or is undergoing. Tsoukleri et al. [90] show that the $2 \mathrm{D}$ peak is shifted to the left (lower frequency) when undergoing tension and to the right (higher frequency) when undergoing compression. 
AFM was first used to study and verify the thickness of graphene. AFM experiments revealed the thickness of a graphene sheet sitting on crystalline graphite to be $.4 \mathrm{~nm}$. This thickness should not change if the graphene is deposited on another substrate, however AFM results have showed that single layer graphene deposited in silica wafers have a thickness of .8-1.2 $\mathrm{nm}$. Only the first layer has this abnormally large thickness as subsequent layers have the expected $.35 \mathrm{~nm}$ thickness. While not confirmed, AFM experiments suggest that the extra thickness may be due to hydrocarbons that have become entrenched between the graphene and the silica during deposition. AFM has also
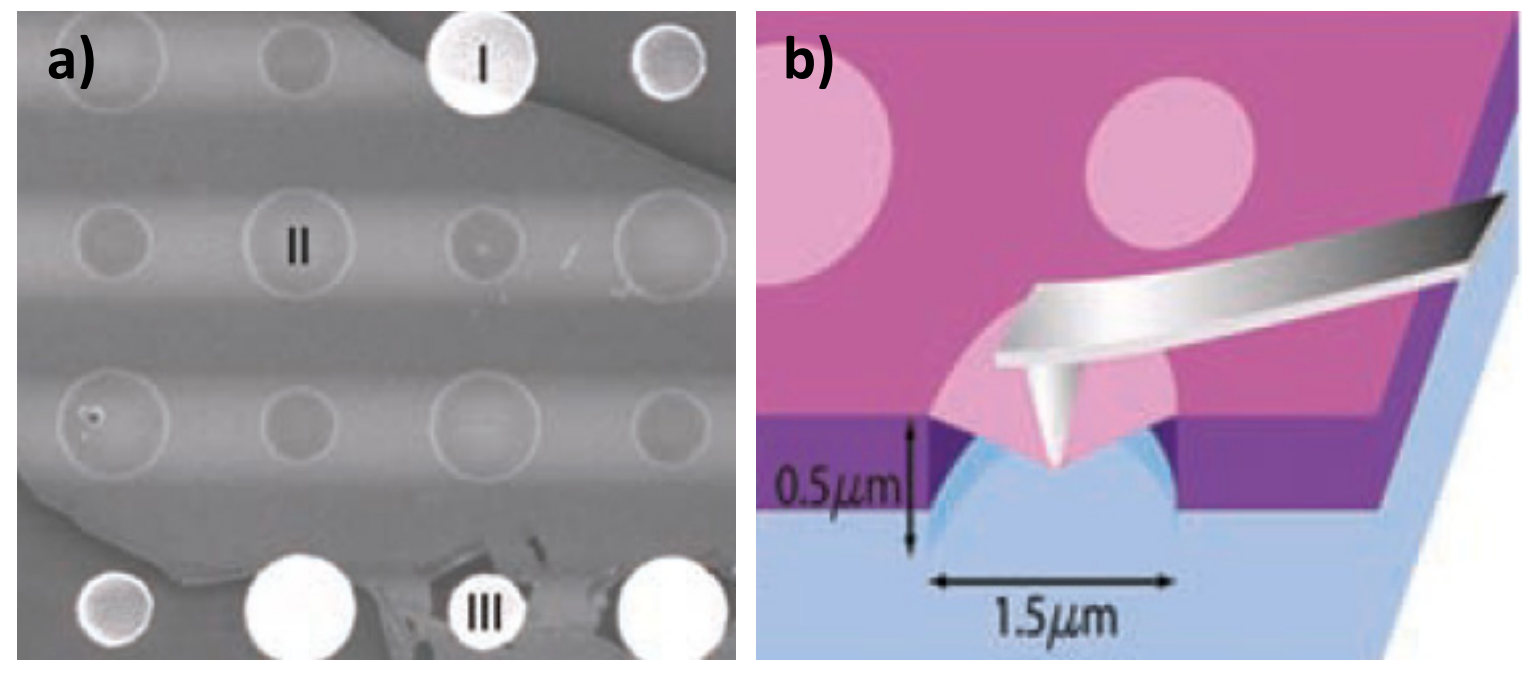

been used to indent a single sheet of graphene to evaluate the strength and elastic response of a graphene sheet. The experimental setup of the AFM nanoindentation is shown in Fig. 2.5. Single layer graphene is grown on a silica substrate with strategically placed cavities as shown in Fig. 2.5a. The cavities causes parts of the graphene sheet to be suspended and these suspended sections are indented by the AFM as shown in Fig. 2.5b. The graphene sheet is found to have an elastic modulus of $1 \mathrm{TPa}$ and a tensile 
strength of $130 \mathrm{GPa}$ [25]. This AFM indentation study established graphene as the material with the highest strength ever measured.

\section{Carbon Allotrope Reinforced Composites}

Carbon allotropes have been shown to reinforce and enhances the properties of polymer, metal, and ceramic matrices. The first allotrope that became widely popular as a reinforcement was carbon fibers and many methods were developed to obtain homogenous composites. Reinforcement of tantalum carbide by addition of carbon fibers was explored in the late 1970s by Gibson et al [91]. Integration of carbon fibers into TaC without damage of the fibers involved many steps such as multiple carbon infiltrations, sucrose decomposition, and treatment of preforms by pressure and heat [91]. Next generation carbon reinforced composites revolve around the use of nano-carbon

allotropes such as carbon nanotubes and graphene. The ease of dispersion and survival of the secondary phase are critical to producing uniform and reliable samples. In the following section, the properties and strengthening mechanisms of CNT and graphene composites are discussed. The section concludes by providing the motivation and reasoning behind using graphene nanoplatelets (GNP) over CNTs or single layer graphene to reinforce $\mathrm{TaC}$ in this thesis.

\subsection{CNT Reinforced Composites}

Carbon nanotubes have successfully reinforced a variety of metal and ceramic matrices such as copper, aluminum, hydroxyapatite, and tantalum carbide. CNTs have 
enhanced the tensile strength, elastic modulus, wear resistance, thermal conductivity, electrical conductivity, and corrosion resistance of metal matrix composites [92]. Corrosion is believed to improve due to the inertness of CNT and their ability to fill up high energy pores. CNT increase thermal and electrical conductivity as CNT have some of the highest thermal conductivities ( $\sim 3000 \mathrm{~W} / \mathrm{mK}$ [93]) and current carrying densities $\left(\sim 4 \times 10^{9} \mathrm{~A} / \mathrm{cm}^{2}\right.$ [94]) of any material. Wear resistance increases as CNT reduce contact between the matrix and the wear counter surface. CNT also provide a lubricating effect that lowers the coefficient of friction in CNT reinforced composites. Improvement of mechanical strength is discussed in more detail below.

CNT improve strength of composites as the load is transferred by the CNT-metal interface and by shearing stress between the two phases. As the length of CNTs increases, the surface area available for load transfer between the matrix and the CNT increases and thus longer CNTs are better reinforcers. It has also been observed that a carbide may form in the CNT-metal interface providing additional interfacial phenomena. Formation of limited amounts of carbides such as $\mathrm{Al}_{4} \mathrm{C}_{3}$ has been shown to improve load transfer in the matrix promoting an increase in strengthening [95]. The carbide will also experience a shearing force and when it yields the CNTs are pulled out. This process strengthens and dissipates energy in the metal matrix composite. Excess formation of carbides can lead to embrittlement of the composite. This phenomenon can only occur in Multi-walled carbon nanotubes (MWCNT) as a single walled CNT would be destroyed upon formation of the carbide. MWCNT have the additional energy dissipation mechanism that arises from shear stress between CNT tubes and this further enhance tensile strength in composites 
[96]. Tensile strength increases most dramatically with addition of $1-2$ volume percent of CNT, the strengthening effect increases more gradually between $2-5$ volume percent CNT [95].

The Young's modulus of metal matrix composites has also been enhanced by CNT reinforcement. CNTs increase the Young's modulus of composites as they have one of the highest elastic moduli of all materials (350-970 GPa [97]). CNTs usually have lengths of a few microns and thus the matrix becomes stiffer at localized micron-scale regions. This local stiffening translates into stiffening of the bulk composite material. The high elasticity of CNT is along the span of CNTs and therefore anisotropic behavior can be induced in the matrix if the CNTs become aligned during processing. If CNTs are aligned, then the direction perpendicular to the span of the CNTs will be weaker than that parallel to it. Dispersion techniques should seek to homogenously distribute the CNT while maintaining some randomness in the $\mathrm{CNT}$ orientation. Processing techniques such as extrusion orient CNTs in one direction and elastic moduli along the extrusion direction have been observed to be higher [98]. Increase in Young's modulus is most dramatic with an addition of .5 - 2 volume [95] percent CNT and the rate of increases continues to drop with increasing CNT content.

Ceramic matrices have also exhibited strengthening by addition of CNTS. In ceramic matrices however, the increases in toughness is of greater importance and CNTS have shown promise in this endeavor as well. CNTs have been used to reinforce a variety of ceramics including such as hydroxyapatite (HA) [99] and tantalum carbide [69]. Reinforcement of an UHTC matrix such as $\mathrm{TaC}$ offers the additional challenge of 
surviving the extreme processing conditions required to consolidate $\mathrm{TaC}$. $\mathrm{HA}$ and $\mathrm{TaC}$ matrices reinforces with CNT and consolidated by spark plasma sintering (SPS) display higher densification and fracture toughness which are attributed to a variety of CNT toughening mechanisms. Presence of CNT enhances densification because the high thermal conductivity enables efficient heat transfer throughout the starting powder during processing. $\mathrm{HA}$ and $\mathrm{TaC}$, like most ceramics, have low thermal and electrical conductivities and this can result in a thermal gradient along the sintering axis. The temperature will be highest at the periphery of the sample due to the proximity to the graphite die which provides the current that heats the sample. This results in a densification gradient in the sample with the center of the sample having the lowest densification. The high thermal and electrical conductivity of CNT reduces the thermal gradient during processing and thus more uniform densification is achieved. CNT reinforced $\mathrm{HA}$ and $\mathrm{TaC}$ also exhibit smaller grain sizes than their respective unreinforced matrices. CNT are believed to inhibit grain growth by pinning grains and reducing their mobility. The improved densification, lower grain size, and high strength of CNT leads to an increase in the Young's modulus of the HA and TaC composite reinforced with CNT. 
Fracture toughness of HA-CNT and TaC-CNT composites are evaluated by indenting polished cross sections and measuring the resulting cracks in order to utilize Anstis equation for Mode I fracture toughness $\left(\mathrm{K}_{\mathrm{IC}}\right)$. CNTs increase the fracture toughness of $\mathrm{TaC}$ and $\mathrm{HA}$ by $60 \%$ and $92 \%$ respectively $[69,99]$. CNT improve fracture toughness by deflecting cracks and thus inhibiting crack propagation. CNT also inhibit crack propagation by forming bridges such as that shown in Fig. 2.6 that provide a resistance to crack formation and propagation. Lahiri et al [99] quantify resistance to crack propagation induced by CNT by comparing the energy required to fracture HA and the energy required to shear a CNT out of the HA matrix. The energy required to fracture HA is $1 \mathrm{~J} / \mathrm{m}^{2}$ [100] while the energy required to shear a CNT out of the HA matrix is 1.5 $22 \mathrm{~J} / \mathrm{m}^{2}$ [99] where the large variation is a result of the varying degrees of CNT pull out observed. Since the energy for shearing a CNT from the HA matrix is greater than that

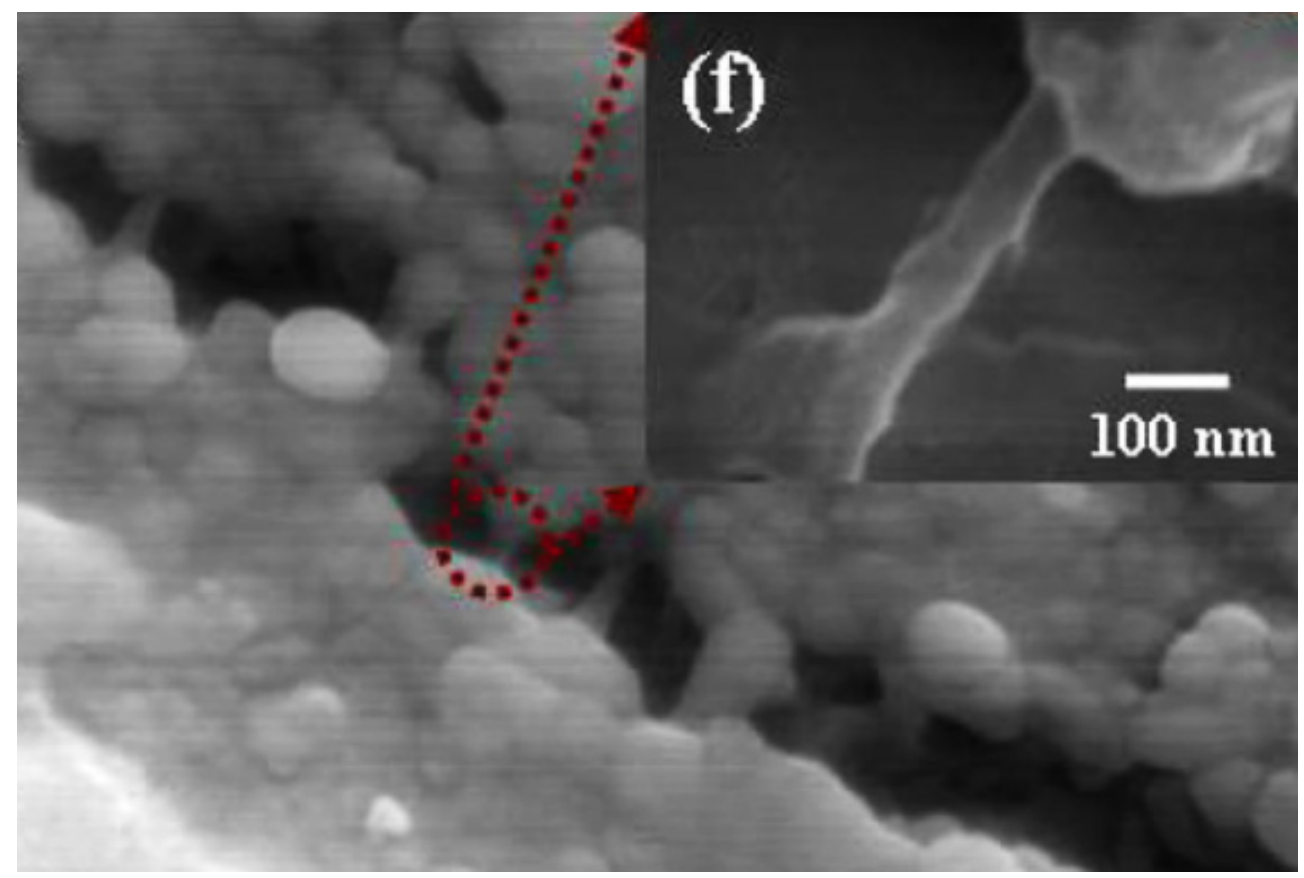


for fracturing HA, a crack propagating through the HA matrix will not necessarily have the energy to break the CNT-HA interface and thus the crack will either deflect in order to find a lower energy path or it will halt propagating. Some cracks will have enough energy to break the CNT-HA bond and pullout the CNT but this decreases the strain energy release rate and thus crack propagation is still inhibited.

The effectiveness of CNTs in both metal and ceramic matrix composites depends on the dispersion of CNTs, the survival of CNTs during processing, and the interface between the matrix and the CNTs. Dispersion of CNTs becomes increasingly challenging as the CNT content is increased as is evidenced by the emergence of CNT clusters at high CNT concentrations. Improper dispersion and CNT clusters are the main factors contributing to lower enhancement of properties at high CNT concentrations. Processing can improve dispersion to some extent, e.g., hot extrusion has demonstrated ability to

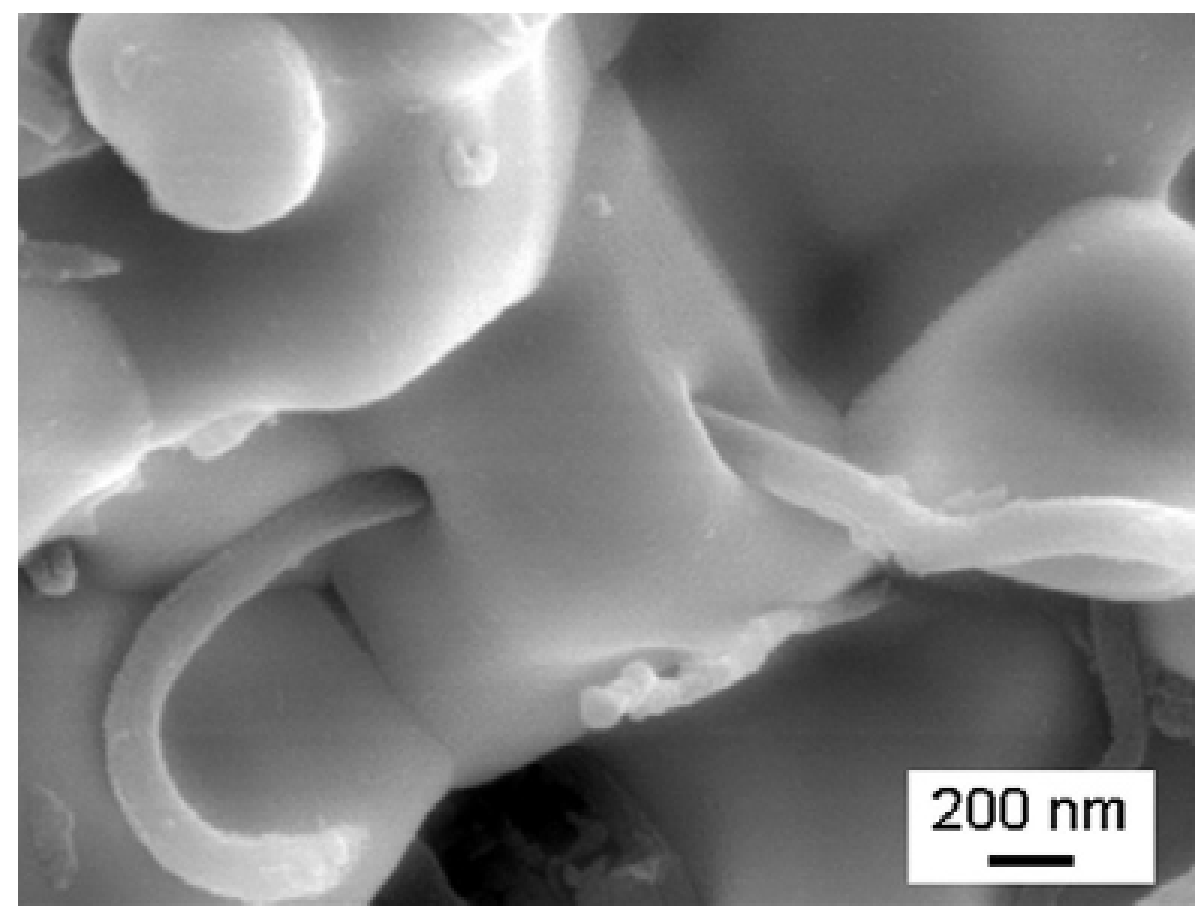


break apart CNT clusters [95]. The processing used must also not be so severe that it causes extensive damage to CNTs. Composites reinforced with longer CNTs have demonstrated superior performance in inhibited grain size, increasing strength, and increasing fracture toughness in TaC-CNT composites [69]. Processing techniques utilizing ball milling can cause long CNTS to become damaged and fracture into smaller CNT segments which are not as effective as longer CNTs. High pressure processing can lead to CNT fracturing and formation of graphitic carbon [69]. The interface between CNT and the matrix is critical as strong interfacial bonding enables better load transfer and hence higher strengthening. A stronger interface increases the energy required for CNT pullout thus increasing the effectiveness of the CNT. A CNT imbedded in a TaC grain, shown in Fig.2.7, provides an excellent CNT-TaC interface and promotes effective load bearing.

\subsection{GNP Reinforced Composites}

Recently with the emergence of graphene and its outstanding mechanical, electrical, and thermal properties, graphene nanoplatelets have been explored as reinforcement of composite materials. Most of the studies have focused on polymer and matrix composites, however recently some studies have been done metal matrix as well as alumina and silicon nitride based composites. In the this subsection graphene nanoplatelet reinforced metal matrix composites are briefly discussed, followed by a more in depth look at alumina and silicon nitride composites as the mechanisms in these ceramic matrices are more relevant to this thesis. 
Graphene reinforced metal matrix composites are perhaps the least explored of the graphene reinforced composites. As with CNTs, the uniform dispersion of graphene is a major challenge in developing graphene reinforced metal matrix composites. One approach to decreasing graphene agglomeration has been to utilize metal nanoparticles than can sit in between graphene sheets in order to prevent stacking of multiple sheets [101]. Metal matrices are likely to react with graphene and thus most composites so far have been consolidated at low temperature in order to reduce reaction products [102]. One recent approach that appears promising in effectively dispersing graphene nanoplatelets in an magnesium metal matrix involves a 2-step process involving liquid state ultrasonication and solid state mechanical stirring [3]. The process begins with an ultrasonicated liquid melt of $\mathrm{Mg}$ at $700{ }^{\circ} \mathrm{C}$. GNP are inserted into the melt and then ultrasonicated for 15 minutes. This melt is formed into a thin plate $(6 \mathrm{~mm})$ and then a friction stirring process is applied on the plate. Dispersion is seen to improve further by the stirring process as graphene clusters in the otherwise homogenous Mg matrix are eliminated. In addition, this process does not result in any reaction products between $\mathrm{Mg}$ and graphene. Hardness of magnesium composite increases by $78 \%$ with addition of 1.2 volume percent GNP [103].

Several studies have been done on alumina [104-106] and silicon nitride [107109] based composites reinforced with GNP in order to improve mechanical, thermal, and electrical properties of the ceramic. In the alumina matrix studies graphene sheets are obtained by processing graphite by ball milling or ultrasonication. Graphene sheets in graphite are held together by weak van der waals forces and thus the force needed to 
sheer of graphene sheets can be provided by these methods. The graphene sheets are exfoliated from the graphite and mixed with alumina in the same procedure in order to disperse the graphene sheets into the alumina matrix. These methods are effective and less costly and less time consuming than exfoliating graphene beforehand by other mechanical or chemical techniques. Ball milling of graphite however has the drawback of producing other potentially unwanted carbon structures such as nanoribbons [105].The resulting alumina-graphene sheet powders are sintered using an SPS machine with parameters of $60 \mathrm{MPa} / 1400{ }^{\circ} \mathrm{C}$ [105], $60 \mathrm{MPa} / 1300{ }^{\circ} \mathrm{C}$ [104], and $50 \mathrm{MPa} / 1300{ }^{\circ} \mathrm{C}$ [106].

In the silicon nitride studies the GNP are either prepared beforehand by reduction of graphite oxide or bought commercially. Obtaining GNP before dispersion into the matrix allows for a more thorough characterization of the quality and uniformity of the GNP to be used. Ramirez et al [108] ultrasonicate the GNP in isopropyl alcohol in order to promote exfoliation and subsequently add the ball milled $\mathrm{Si}_{3} \mathrm{~N}_{4}$ powder into the alcohol in order to disperse the GNP in the $\mathrm{Si}_{3} \mathrm{~N}_{4}$ matrix. Walker et al [107] ultrasonicate the $\mathrm{Si}_{3} \mathrm{~N}_{4}$ and GNP powders separately in water before mixing them and ultrasonicating them further. The ultrasonicated mixture is then ball milled using alumina media. The water is evaporated after the ball milling process. Dusza et al [109] do not ultrasonicate the GNP and directly proceed to ball mill the GNP and the Si3N4 together. Ball milled mixture includes water and the surfactant polyethyenglycol (PEG). The milling media is made of zirconia and milled powders are found to have zirconia contamination. These powders are oxidized in order to remove PEG and then consolidated using hot isostatic pressing at $1700{ }^{\circ} \mathrm{C}$. The $\mathrm{Si}_{3} \mathrm{~N}_{4}$-GNP powders prepared by ultrasonication and ball 
milling by Ramirez et al [108] and Walker et al [107] are consolidated by SPS using parameters of $50 \mathrm{MPa} / 1625{ }^{\circ} \mathrm{C}[108]$ and $35 \mathrm{MPa} / 1650{ }^{\circ} \mathrm{C}$ [107] respectively.

Graphene like CNTs have a very high thermal conductivity $\left(5.1 \times 10^{3} \mathrm{~W} / \mathrm{MK}\right.$ [110]) and are therefore expected to improve the thermal conductivity of the composite they reinforce. An improvement of thermal conductivity will decrease thermal gradients during processing. Uniform heating of the samples during sintering leads to an improvement in densification and microstructural uniformity of the bulk composite. Microstructural uniformity translates to uniform properties which is essential for reliability in high performance materials. Graphene also have excellent electrical properties with single layer graphene having an electron mobility of $2.0 \times 10^{5} \mathrm{~cm}^{2} / \mathrm{V} \cdot \mathrm{s}$ [111]. The electrical conductivities of $\mathrm{Al}_{2} \mathrm{O}_{3}-\mathrm{GNP}$ composites has been measured to be $172-5709 \mathrm{~S} / \mathrm{m}[6,8]$ depending on the GNP content. The $5709 \mathrm{~S} / \mathrm{m}$ value was achieved by the addition of 15 volume percent GNP, this improvement is $170 \%$ higher the that achieved by reinforcing $\mathrm{Al}_{2} \mathrm{O}_{3}$ with MWCNTs. Ramirez et al [108] enhance to electrical conductivity of $\mathrm{Si}_{3} \mathrm{~N}_{4}$ composites by adding 25 volume percent of GNP. The electrical conductivity of these samples was $4000 \mathrm{~S} / \mathrm{m}$ which also outperforms the improvements achieved by MWCNT. It is also observed by Ramirez et al [108] that GNP becoming oriented during the SPS processing and electrical conductivity is much higher in the direction perpendicular to the SPS pressing axis. Orientation of platelet-like particles is not uncommon during compression and has previously been observed in SiC platelet reinforced $\mathrm{Al}_{2} \mathrm{O}_{3}$ composites [112] and graphite platelet reinforced $\mathrm{Al}_{2} \mathrm{O}_{3}$ composites 

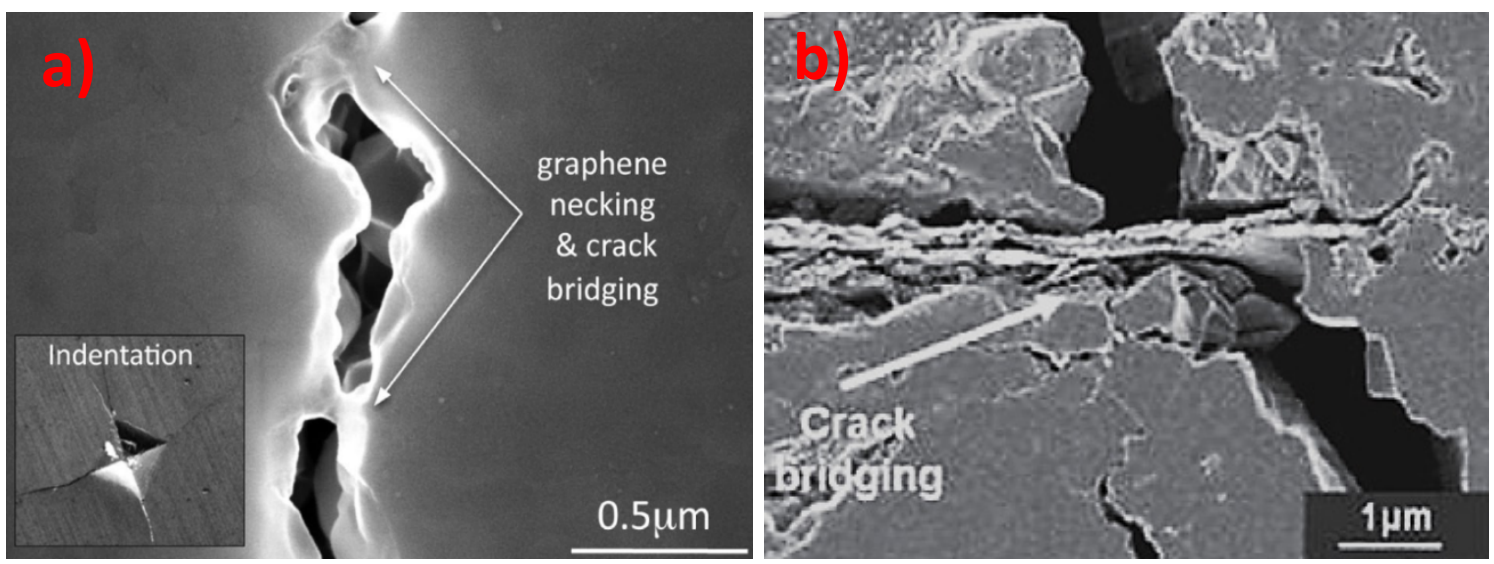

[113]. So far no anisotropic behavior of mechanical properties has been observed in GNP reinforced composites.

As mentioned in the CNT section, improvement of fracture toughness in ceramic materials is of critical importance as it will enable ceramics such as $\mathrm{HA}$ and $\mathrm{TaC}$ to be implemented in emerging orthopedic and aerospace technologies. GNP reinforced $\mathrm{Al}_{2} \mathrm{O}_{3}$ and Si3N4 matrices have shown improvement in fracture toughness of 53\% [106] and $235 \%$ [107] respectively as a result of GNP toughening mechanisms. Walker et al [107] perform high load indentations $(90 \mathrm{~N})$ in order to look inside the resulting cracks and observed the GNP toughening mechanisms. GNP of varying sizes are observed to bridge cracks and forms necks between cracks as observed in Fig. 2.8a and b.

These mechanisms dissipate energy and resist crack propagation which strengthens the material. When cracks attempt to propagate through a graphene sheet they seek a lower energy path and smaller cracks branch away from the main crack as shown in Fig. 2.9. These smaller cracks don't degrade the structural integrity of the material as 


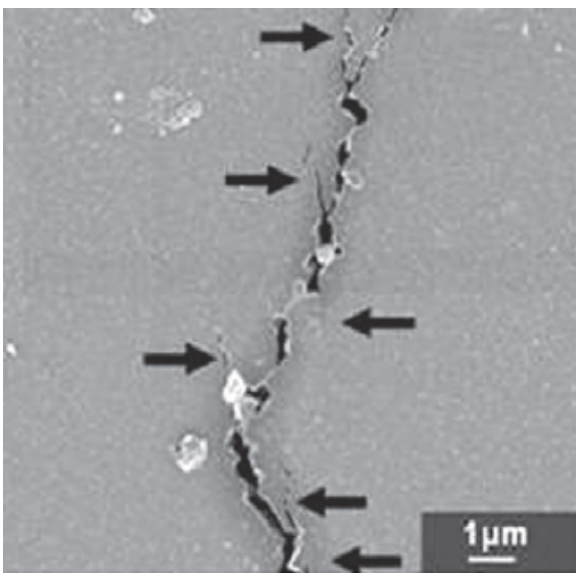

much as the larger cracks as they are further away from the critical flaw size needed for fast fracture. High energy cracks will not be deflected by graphene, however the energy used in pulling put the graphene sheet results in lower energy available for crack propagation.

The addition of graphene also strengthens the ceramic matrix by hindering grain growth $[106,107,109]$. GNPs are observed to wrap around grains as shown in the TEM

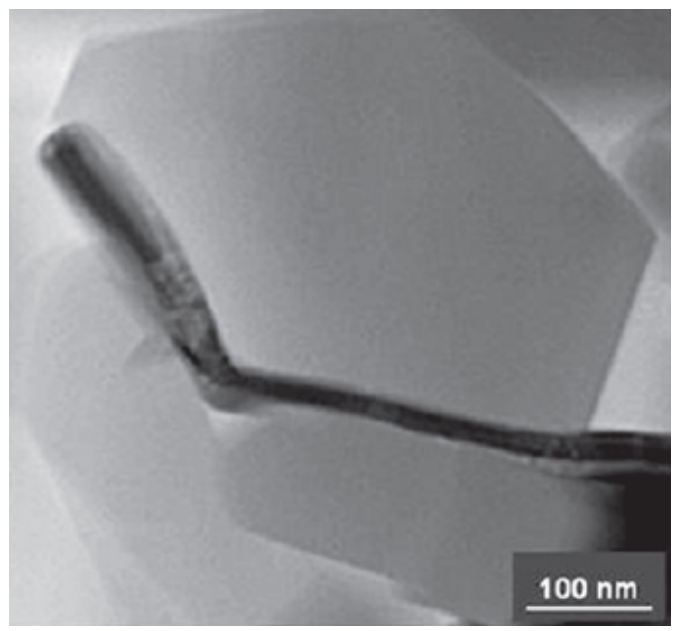


micrograph in Fig. 2.10. Graphene pins the grains physically and acts as a diffusion barrier thus inhibiting grain growth. The hardness of GNP reinforced composites has been measured and mixed results have been obtained. GNP is a soft phase and thus a decrease in hardness would be expected in the absence of grain size reduction. It has been observed that GNP that have been ball milled for longer periods and hence reduced in size lead to finer grains in the consolidated structures [105]. Finer grain sizes are expected to strengthen the material however the consolidated structures were not mechanically tested in that study. More work is needed to see what types and contents of GNP increase hardness and which are detrimental to mechanical strength.

\subsection{GNP vs. CNT}

Graphene nanoplatelets have the potential to be superior reinforcers than carbon nanotubes in several aspects. To date, GNP and CNT performance in composites has not been directly compared in many studies. One of the few studies to compare GNP and CNT directly involved the comparison of mechanical properties of epoxy composites that were reinforced with GNP, SWCNT, or MWCNT [114]. The volume percent was kept constant at .1 weight percent and it was demonstrated that GNP reinforced composites exhibited superior mechanical properties. GNP improves the Young's modulus by $31 \%$ while SWCNT only led to a 3\% increase. Tensile strength of GNP reinforced epoxy composite improved by $40 \%$ while MWCNT reinforced composites improved by $14 \%$. Fracture toughness was also superior for GNP reinforced composites, 53\% improvement by GNP as compared to $20 \%$ improvement by MWCNT. More studies are needed to 
verify whether these results hold true other polymer, metal, and ceramic matrices, however, GNP have specific attributes that make them superior to CNTs as will be discussed in detail below.

Some might question how can graphene be a superior reinforcement as compared to CNT, given the large similarities between the two materials. The critical factor that makes GNP superior is in the one property that is very different in these two materials their geometry. The thin flat nature of graphene and GNP gives it a 2D nature while CNTs have a 1D nature. This 2D nature gives GNP the potential to have more efficient strengthening and toughening mechanism than $\mathrm{CNT}$, particularly in ceramic matrix composites where there is little chemical interactive between matrix and carbon reinforcement. CNT have been shown to inhibit grain growth by pinning grains which slows their movements. GNPs have this same pinning mechanism however in their case the GNP have been shown to wrap around the entire grain. Wrapping around the grain not only pins the grains more effectively but the graphene also acts as a diffusion behavior so smaller grains cannot ripen into larger grains. The wrapping of grains by GNP that inhibits grain growth is also a toughening mechanism not present in CNT. Walker et al [107] have observed that these wrapped sheets of graphene can provide resistance to propagating cracks in three dimensions.

In addition graphene has the same toughening mechanism observed in CNT however the higher surface area of graphene and GNP makes toughening mechanisms more effective. In CNT, cracks are bridged and obstructed by a single CNT or possibly a bundle of a few CNTs. It is inherently easier for a crack to propagate around a 1D 
structure such as CNT than it for a 2D sheet of graphene. The increased area of GNP also provides more material and thus higher strength bridges and necks. The increased area of GNP is so effective because the area in contact with the matrix is higher than in CNTs. Higher contact area enables the GNP to more effectively bear the applied load on the composite. Higher contact area also means the shear force needed to pull a GNP or graphene sheet out will be higher than that needed for CNT. Higher resistance to sheet pullout translates to a higher energy needed for a crack to propagate in the matrix and thus GNP toughening mechanisms are more effective.

The 2D nature of GNP also enables allows for easier dispersion and processing. GNPs are easier to homogenously disperse in a matrix even with simple methods such as wet chemistry. GNP are superior over CNTs as CNT tend to become entangled and bundled up leading to agglomerates which makes higher concentration reinforcements inefficient. GNP are also superior to single layer graphene when it comes to GNP as they are thick enough to avoid becoming curled up or rolled up into CNT like tubes as occurs with single layer graphene. The 2D nature of graphene also lends itself to sintering processes that employ high pressures. As mentioned before, platelet-like materials may become oriented in one direction during axial compression however the damage to them is minimized as they will reorient themselves before the fracture. CNTs on the other hand are more sensitive to high pressure processing because of their hollow tubular structure and they usually fracture and buckle during processing. CNT length is critical to efficient reinforcement and therefore the formation of shorter CNTs as a result of processing is highly unfavorable. 
GNPs are advantageous over CNT from an economical and industrial point of view. GNP can be produced by much simpler methods than CNT and thus more readily available. Simple fabrication methods allow for a higher degree of quality and dimensional control as compared to CNTs. CNTs are mostly grown by CVD processes that have not yet established consistent parameter-property relationships. GNPs also have high potential for quick industrial implementation as they are considerably cheaper than CNT. GNP can be bought in bulk form for less than $\$ 200$ per kilogram which is several times cheaper than industrial grade CNTs. Further investigation of GNP reinforced composites is needed to confirm that GNP are superior to CNTs from both an economical and performance standpoint.

\section{Spark Plasma Sintering}

The use of spark plasma sintering (SPS) in this Thesis is employed due to its demonstrated capability of consolidating nanoceramics to full or near full density with limited grain growth. The achievement of full densification without inducing large grain growth of the nano-powders is the most critical aspect of sintering and consolidating nanoceramics. Spark plasma sintering is a sintering process that combines an applied pressure with a pulsed electric current that flows through the powder being sintered. The sintering mechanisms induced by spark plasma sintering allow for rapid consolidation along with lower sintering temperatures than conventional sintering techniques [115]. Spark plasma sintering techniques have attracted much attention because of its unique 
sintering mechanisms and its ability to form bulk materials that are challenging to consolidate.

Ceramic materials, including ultra high temperature ceramics, can be consolidated to full density by conventional methods such as hot pressing. Hot pressing consists of pressing ceramic powders together at high temperatures for a sustained period of time, over an hour at times. The high temperature is needed in order to initiate densification mechanisms such as grain boundary diffusion, surface diffusion, and volume diffusion [116]. The high pressure leads to plastic deformation and creep which are additional sintering mechanisms [116]. The driving force of sintering is the lowering of surface energy as described in Eqn. (2.11) below:

$$
\Delta\left(\sum \gamma_{i} A_{i}\right)=\gamma_{s v} \Delta A_{s v}+\gamma_{s s} \Delta A_{s s}
$$

The LHS of Eqn. (2.11) represents the reduction in surface energy and it can be seen that lowering the interfacial surface area decreases the surface energy. Sintering decreases surface area and specifically interfacial area between pores and grains or particles by forming necks and joining particles which eliminates the pores. The higher the initial interfacial area present, the higher the potential or driving force of sintering will be. The use of nanoparticles is thus favorable as they inherently have higher specific areas and thus a larger driving force. As, the driving force for sintering decreases, the time needed to complete densification grows large. During hot pressing of nanoceramics grain growth occurs due to the extended exposure to high temperatures and the nanoparticle size is lost. The task of maintaining small grain size and achieving high densification is 
evidently and inherently challenging because densification and grain growth occur at the same time. Large grain sizes are undesirable as they lead to lower strength materials. If hot pressing is done at lower temperatures or for lower periods of time then porosity is inevitable and this also leads to a loss of strength.

Spark plasma sintering attempts to balance the competing sintering and grain growth mechanisms by applying high heating rates, that are achieved due to the pulsing current, alongside an axial compression. The applied pressure enhances densification by inducing plastic deformation as in other conventional pressure assisted sintering methods. The pulsing current heats up the die holding the powder to be sintered and in conductive samples the electric current will pulse through the powder as well. There are two primary mechanisms that are due solely to the use of a pulsed electric current and they are schematically illustrated in Fig. 2.11. The electrical resistance of the material leads to Joule heating when a current is applied and this is the source of heating during SPS [28].

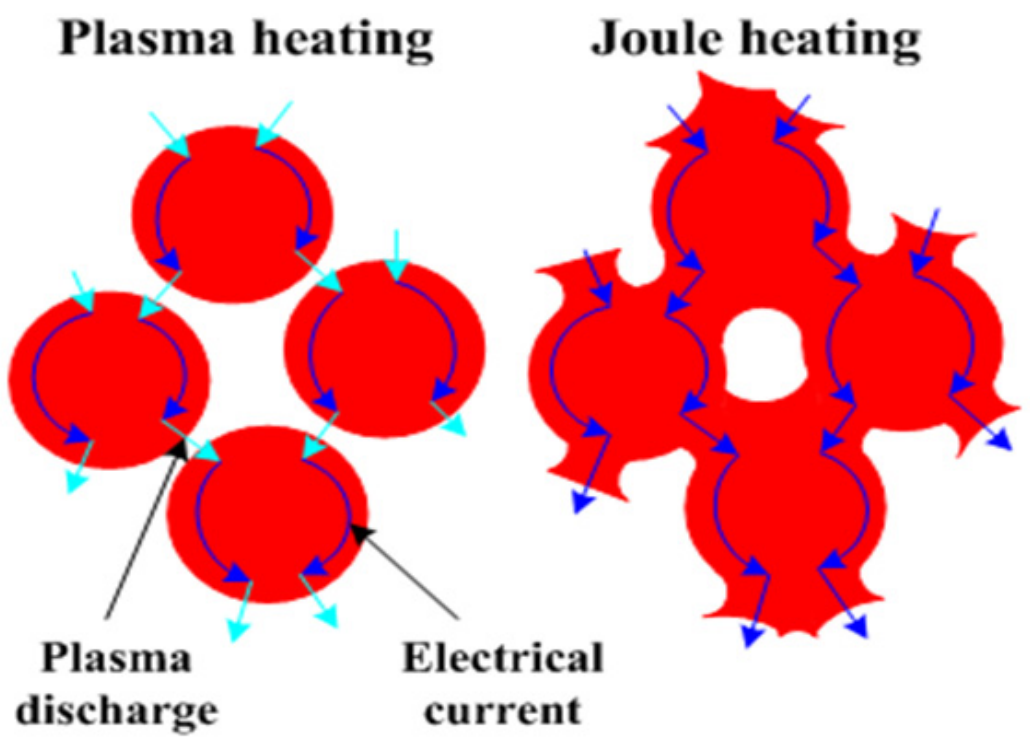


This is advantageous over conventional sintering methods where the heat is supplied by some external source which means heat must be transferred to the samples. In the SPS, the heat transfer effects are minimized because the heat is generated internally due to the Joule heating caused by the electric current. Samples with higher electrical conductivity do have the advantage of more uniform electrical current distribution as the electrical current will be highest at the periphery of the powder since it is in contact with the graphite die which is highly conductive. Conductive samples will conduct the current efficiently and thus heating of the sample will also be uniform. Nonconductive samples will have a large thermal gradient than conductive samples and thus will be more susceptible to variations in microstructure and properties.

The other unique mechanism due to the pulsed electrical system is behind the name "Spark Plasma" in the SPS name. The pulsing electric current is believed to generate plasma in in the form of micro-discharges originated from impurities in the particles surfaces. This plasma has the dual effect of cleansing the particles of impurities and of forming "necks" between particles. This necking phenomenon aids in the efficient transfer of electric current and heat throughout the samples. This is especially helpful in non-conductive samples where Joule heating will be highest at the peripheries. The cleansing of particle aids in sintering as it leads to better bonding between particles and decreases presence of impurities. Low melting point impurities will not resolidify and instead will leave the sample leading to outgassing in the sample. If the impurities present are of high melting temperature then the likelihood of a plasma forming decrease and the more likely mechanism is the formation of electrical sparks between particles. The 
pulsing nature of the current, often as frequent as 1 millisecond [117], would make the formation of sparks possible especially in highly conductive samples. These sparks would have the same effects removing impurities and enhancing neck formations as with the plasma. These electrical current mechanisms however are disputed and have led some researchers to refer to SPS as pulsed electric current sintering (PECS) or field assisted sintering (FAST), thus omitting any references to sparks or plasma.

A basic schematic of an SPS machine is shown in Fig. 2.12. The powder is placed inside a graphite die which is then compressed using a graphite punch which serves the dual purpose of applying pressure and conducting electricity. These dual roles mean that

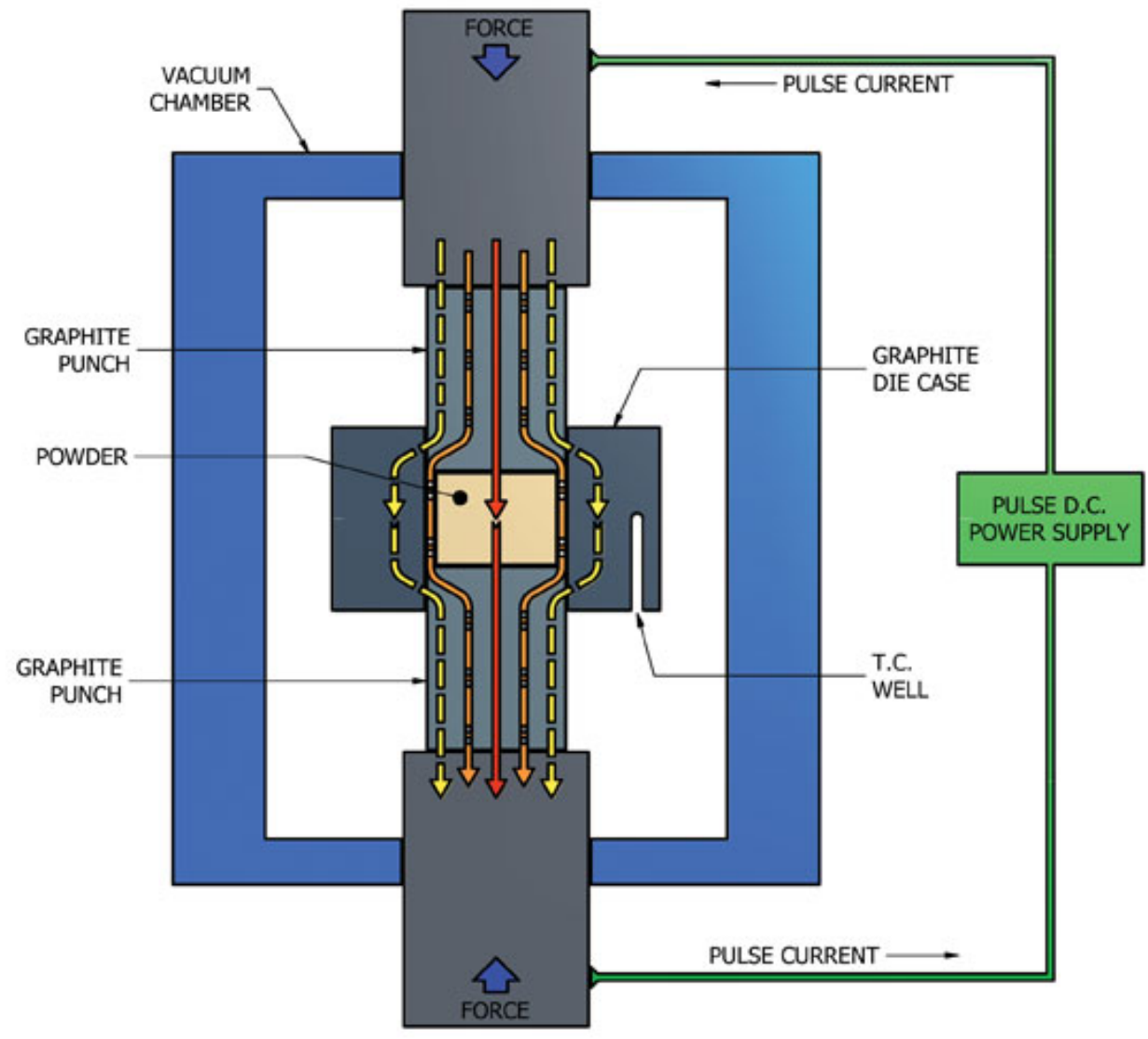


there are limitations to the amount of heat and pressure that can be generated during sintering. Graphite is the most widely used die because of its high conductivity and high thermal resistance. This allows for high currents and high temperatures to be generated. However, the pressure applied cannot exceed much more than $100 \mathrm{MPa}$ otherwise the graphite die will fracture. Also, the strength of graphite decreases at higher temperatures so the feasible applied pressures using a graphite die at high temperatures will be lower than those at lower temperatures. Different dies can be used but there will always be limitations. Dies which can sustain very high pressures and temperatures are available, such as $\mathrm{SiC}$ dies, however in addition to being much more expensive they are not nearly as conductive as graphite.

It can been sheen from Fig. 15 that temperature is measured by inserting a thermocouple into one of the graphite dies. This illustrates one of the short comings with generating high temperatures by Joule heating, which is that the control of the applied temperature is not as simple as in a furnace environment. A thermocouple or an optical pyrometer can only measure the temperature in the die and not the sintered powder directly. The high temperature is generated internally and thus the measured temperature will be lower by as much as $250{ }^{\circ} \mathrm{C}$ at high temperatures [118]. Another challenge in SPS processing is the scalability of the process without having adverse effects on the sintering environment. Experimental and computational studies have indicated that while properties such as density and microstructure can be consistently reproduced in a given SPS machine, significant deviations can be present using different machines $[119,120]$. The implementation of larger sample sizes has also shown an increased variation in 
temperature profiles throughout the sample [120]. The larger sample leads to a higher temperature gradient and this could result in significant property variations between the center of the sample and the periphery. Temperature variation can be suppressed by using lower heating rates, however higher heating rates are crucial to SPS performance as they allow for quick sintering times which reduces grain growth as previously discussed.

As mentioned before, SPS has been used to consolidate nanoceramics to high density with limited grain growth. Nanoceramic composites have also been sintered using SPS and one the secondary phases that has been most widely studied recently are carbon nanotubes $(\mathrm{CNT})$. CNTs are an excellent candidate for reinforcing ceramic composites that are consolidated by SPS as they have excellent thermal and electrical conductivity. CNTs increase the conductivity of the composite powder and thus sintering will improve in ceramics as ceramics are typically insulators. Improved electrical conductivity will decrease thermal gradients in the samples and lead to more uniform properties. As alluded to in Section 2.3, CNT become damaged during processing however their survival in extreme SPS processing conditions is of great significance. Several studies have been conducted on the feasibility of CNT survival and structure retention during high pressure and high temperature SPS processing. Bulk CNT and MWCNT samples have been consolidated by SPS using pressures ranging from $7 \mathrm{MPa}$ to $100 \mathrm{MPa}$, and temperatures ranging from $1100{ }^{\circ} \mathrm{C}$ to $1600{ }^{\circ} \mathrm{C}$ [121-124]. Laurent et al [123] sintered double-walled CNTs by SPS at $1100{ }^{\circ} \mathrm{C}$ and $100 \mathrm{MPa}$ and based on Raman spectroscopy and electron microscopy they assessed that no significant damage had been incurred by the CNT. Studies on MWCNT have shown the formation of other carbon phases such as 
amorphous carbon, diamond, and graphene [121, 122]. MWCNT samples sintered at 80 MPa had very few CNT remaining after the processing as most had been converted to diamond [121]. MWCNT sintered at $7 \mathrm{MPa}$ and at the same temperature $\left(1500{ }^{\circ} \mathrm{C}\right)$, experienced damage in the form of kinks, collapse, and intertubular bonding, however they retained their orientation, geometry, and crystallinity [122]. The lower pressure used in this study are likely the reason the CNT survive as the tubular structure of CNTs is vulnerable to buckling and fracturing when a high axial stress is applied.

\section{UHTC Oxidation Studies and Methods}

Ultra high temperature ceramics (UHTC) materials would be used in high temperature aerospace applications and it is therefore important to characterize the oxidation behavior of these materials. While the extreme upper temperature limit of a material is its melting point, the actual service condition shouldn't reach this level. Material properties will change as the temperature changes and it thus important to

evaluate how thermal and mechanical properties change at increasing temperatures. A material will have a critical temperature limit where properties deteriorate and it can no longer meet performance requirements for certain applications. Both conventional furnace testing and aerothermal oxidation studies are important as they provide different pieces of information which complement each other. 


\subsection{Conventional Furnace Testing}

In addition to studying oxidation behavior, furnace testing can be used to perform in situ mechanical testing of properties such as flexure strength. Oxidation behavior is typically characterized by observing the microstructural evolution, the structural integrity, and the change in weight. If the sample is still structurally stable then mechanical properties can be evaluated in situ or after the high temperature exposure is completed.

Furnace oxidation studies have been conducted mostly on $\mathrm{ZrB}_{2}-\mathrm{SiC}[37,40,125$ 128] based UHTC materials as well a few studies on $\mathrm{HfB}_{2}$ [30], and $\mathrm{TaC}$ [48]. ZrB $2-\mathrm{SiC}$ composites were oxidized at temperatures ranging $700{ }^{\circ} \mathrm{C}$ to $2000{ }^{\circ} \mathrm{C}$ by two separate teams $[125,37]$ and the behavior observed was consistent. The addition of $\mathrm{SiC}$ leads to the formation of a glassy layer that together with the $\mathrm{B}_{2} \mathrm{O}_{3}$ protects the porous $\mathrm{ZrO}_{2}$ oxide from further oxygen infiltration. The liquid boria and silicate seal the pores and grain boundaries of the oxide layer. Oxidation occurs most rapidly along grain boundaries as the high energy boundary essentially provides a route for oxygen to diffuse downwards. $\mathrm{ZrB}_{2}-\mathrm{SiC}$ composites experience negligible oxidation below $700{ }^{\circ} \mathrm{C}$ and the $\mathrm{SiC}$ particles do no become actively oxidized until a temperature of about $1600{ }^{\circ} \mathrm{C}$. The oxidation of $\mathrm{SiC}$ results in a porous structure which becomes oxidized rapidly and in turn generates more porous regions. Above $1800{ }^{\circ} \mathrm{C} \mathrm{ZrO}_{2}$ growth increases substantially and dendritic structures are observed post oxidation [125]. Characterization of oxidation behavior above $2000{ }^{\circ} \mathrm{C}$ requires use of arc-jet or other high temperature aerothermal tests. The flexural strengths of $\mathrm{ZrB}_{2}-\mathrm{SiC}$ composite are evaluated in situ at $23{ }^{\circ} \mathrm{C}, 1000{ }^{\circ} \mathrm{C}, 1400{ }^{\circ} \mathrm{C}$, 
and $1600{ }^{\circ} \mathrm{C}$ with soaking times of $20 \mathrm{~min}$. The strength at $1000{ }^{\circ} \mathrm{C}$ is found to be higher than that at room temperature likely because the high temperature allows for healing of cracks and defects generated during machining of the samples. The flexure strength at $1400{ }^{\circ} \mathrm{C}$ is found to be the same as the room temperature strength while the strength of the sample tested at $1600{ }^{\circ} \mathrm{C}$ decreases by $16 \%$ [37].

$\mathrm{ZrB}_{2}$ composites reinforced with $\mathrm{SiC}$ fibers tested at $1200{ }^{\circ} \mathrm{C}$ for $30 \mathrm{~min}$ and their performance was compared to $\mathrm{ZrB}_{2}$ composites reinforced with $\mathrm{SiC}$ particles. The flexural strength of $\mathrm{SiC}$ fiber reinforced $\mathrm{ZrB}_{2}$ composites had higher strength at $1200{ }^{\circ} \mathrm{C}$ than the unreinforced $\mathrm{ZrB}_{2}$ [40]. The lack of improvement in the flexural strength may be due to the $\mathrm{SiC}$ fibers exceeding the critical flaw size and thus acting as crack initiation sites. At $1200{ }^{\circ} \mathrm{C}$ the fibers may begin to oxidize and form silicates which lead to better interfacial bonding between $\mathrm{SiC}$ fibers and matrix. As with the particle reinforced $\mathrm{ZrB}_{2}$ composites, the strength does not change significantly at temperatures in the $1200{ }^{\circ} \mathrm{C}-$ $1400{ }^{\circ} \mathrm{C}$ range.

$\mathrm{ZrB}_{2}-\mathrm{SiC}$ composites are reinforced with $\mathrm{ZrC}$ and oxidized at $1000{ }^{\circ} \mathrm{C}, 1200{ }^{\circ} \mathrm{C}$, and $1400{ }^{\circ} \mathrm{C}$ for up to $60 \mathrm{~min}[126,127]$. The flexural strength of the samples oxidized at $1000{ }^{\circ} \mathrm{C}$ increased with increasing exposure time and levels out when exposed for 30 min. As in the previous $\mathrm{ZrB}_{2}-\mathrm{SiC}$ studies discussed the strengthening is believed to be the result of glassy boria and silicate phases filling cracks and defects in a crack healing process. The increase in volume during oxidation may also have the effect of reducing crack volumes and sizes. At a temperature of $1000{ }^{\circ} \mathrm{C}$ the glassy phase consists mostly of boria. The flexure strength of samples oxidized at $1200{ }^{\circ} \mathrm{C}$ display a higher flexure 
strength than those at $1000{ }^{\circ} \mathrm{C}$ and the peak value is reach after $20 \mathrm{~min}$ instead of $30 \mathrm{~min}$. At this temperature the formation of silica is more prevalent and the fact that the peak strength is reached sooner than at $1000{ }^{\circ} \mathrm{C}$ indicates silica is more effective in healing cracks than boria. At $1400{ }^{\circ} \mathrm{C}$ the maximum flexural strength is the same as that obtained at $1200{ }^{\circ} \mathrm{C}(\sim 660 \mathrm{MPa})$. The peak flexural strength value however is reached after only $10 \mathrm{~min}$ as the higher temperature allows the silica to evolve sooner.

Hafnium diboride based composites consolidated by various methods have been oxidized in conventional furnaces up to temperatures of $2000{ }^{\circ} \mathrm{C}$. $\mathrm{HfB}_{2}-\mathrm{SiC}-\mathrm{TaSi}_{2}$ composites were consolidated by hot pressing and oxidized at $1500{ }^{\circ} \mathrm{C}$ [129]. The flexure strength of this material decreases from $\sim 725 \mathrm{MPa}$ at room temperature to $\sim 460 \mathrm{MPa}$ after being oxidized at $1500{ }^{\circ} \mathrm{C}$. $\mathrm{HfB}_{2}-\mathrm{SiC}$-HfC composites were sintered by reactive hot pressing and oxidized at $1500{ }^{\circ} \mathrm{C}$ [130]. The flexure strength of these materials also decreases after oxidation, from $\sim 775 \mathrm{MPa}$ to $300 \mathrm{MPa}$. $\mathrm{HfB}_{2}-\mathrm{SiC}$ and $\mathrm{HfB}_{2}-\mathrm{MoSi}_{2}$ composites that have been consolidated by spark plasma sintering have been able to retain their flexure strength after similar $1500{ }^{\circ} \mathrm{C}$ heat exposures [131-133]. Bellosi et al [133] produce $\mathrm{HfB}_{2}-\mathrm{SiC}$ composites with flexure strengths of $\sim 600 \mathrm{MPa}$ at both room temperature and $1500{ }^{\circ} \mathrm{C}$. The improvement in oxidation strength is believed to be due to the sintering mechanisms unique to SPS. As discussed in Section 2.4, SPS is believed to remove impurities during the formation of plasma. The $\mathrm{HfB}_{2}$ based composites consolidated by SPS having shown fewer impurities $[131,133]$. The removal of oxide impurities and impurities prone to oxidize is believed to improve the oxidation behavior of $\mathrm{HfB}_{2}$ based composites. 
The oxidation behavior of $\mathrm{TaC}$ based UHTCs is characterized by tracking the mass changes throughout the oxidation cycle using thermal gravimetric analysis (TGA) [48]. The addition of TaB2 enhances mechanical properties as discussed in Section 2.1, however as emphasized before the assessment of the materials performance for aerospace applications is not complete until the oxidation behavior is evaluated. $\mathrm{TaC}$ and $\mathrm{TaC}-\mathrm{TaB} 2$ samples are oxidized at $1500{ }^{\circ} \mathrm{C}$ with mass change information being continuously measured by TGA. The TaC samples form a porous $\mathrm{Ta} 2 \mathrm{O} 5$ oxide which results in a weight gain of $85 \mathrm{mg} / \mathrm{cm}^{2}(14.5 \%)$ [48]. This weight gain is the same as the the weight gain expected for full conversion of $\mathrm{TaC}$ to $\mathrm{Ta} 2 \mathrm{O} 5$ which signals that all of the carbon is lost as carbon monoxide (CO) gases escape from the surface. The TaC-TaB2 sample experiences significant weight gain at $900{ }^{\circ} \mathrm{C}$ while the $\mathrm{TaC}$ do so at $800{ }^{\circ} \mathrm{C}$. The mass gain of the $\mathrm{TaC}-\mathrm{TaB} 2$ samples exhibit lower mass gains than $\mathrm{TaC}$ at temperature below $1350{ }^{\circ} \mathrm{C}$. This improvement in oxidation is attributed to the formation of a protective boria layer from the oxidized TaB2. Such protective layers are analogous to the protective boria and silica layers formed in $\mathrm{ZrB}_{2}-\mathrm{SiC}$ composites discussed extensively above. The $\mathrm{TaC}-\mathrm{TaB} 2$ samples however do not show any improvement over $\mathrm{TaC}$ samples in oxidation behavior above $1500{ }^{\circ} \mathrm{C}$. Oxidation resistance of UHTC materials is needed at temperatures substantially greater than $1500{ }^{\circ} \mathrm{C}$. Oxidation resistance at temperatures above $2000{ }^{\circ} \mathrm{C}$ is needed and in the following section the behavior of UHTCs in such environments is elaborated on. 


\subsection{Aerothermal Testing}

Aerothermal testing is crucial to evaluating the performance of UHTC materials as these materials will be exposed to aerothermal phenomena during actual service. Failure of these materials will be due to a combination of melting, vaporization, sublimation, oxidation, ablation, and spalling caused by the high temperatures and chemical attacks experienced [134]. Aerothermal testing has been conducted mostly on $\mathrm{ZrB}_{2}$-SiC composites [135-139] however some studies have also been conducted on $\mathrm{HfB}_{2}$ [140], HfC [140, 141], and TaC [142] based composites. Aerothermal testing are usually conducted at temperatures over $2000{ }^{\circ} \mathrm{C}$ and usually at high flow speeds, which introduce high shear forces, although no necessarily at the supersonic or hypersonic speeds experienced in actual service conditions.

The materials tested are challenging to consolidate to full density as previously discussed, and therefore machining an ideal shape for aerothermal testing is challenging. UHTC materials have been tested in a variety of shapes as shown in Fig.2.13, some more complex than others. The most promising configurations, as far as simulating actual aerothermal effects experienced are those that mimic shapes used in aerospace designs such as the blunt body and nosecone configurations in Fig. 2.13c and d respectively. As introduced in Section 1.1, future hypersonic vehicle concepts employ very thin leading edges and Fig. 2.14 demonstrates the similarity of the X-51 leading edge and the sharp shaped UHTC material geometry used in several studies [135, 138, 139]. Employing standard aerodynamic shapes for UHTC testing enables the evaluation of the microstructure at different points along the surface profile. The microstructure at the 

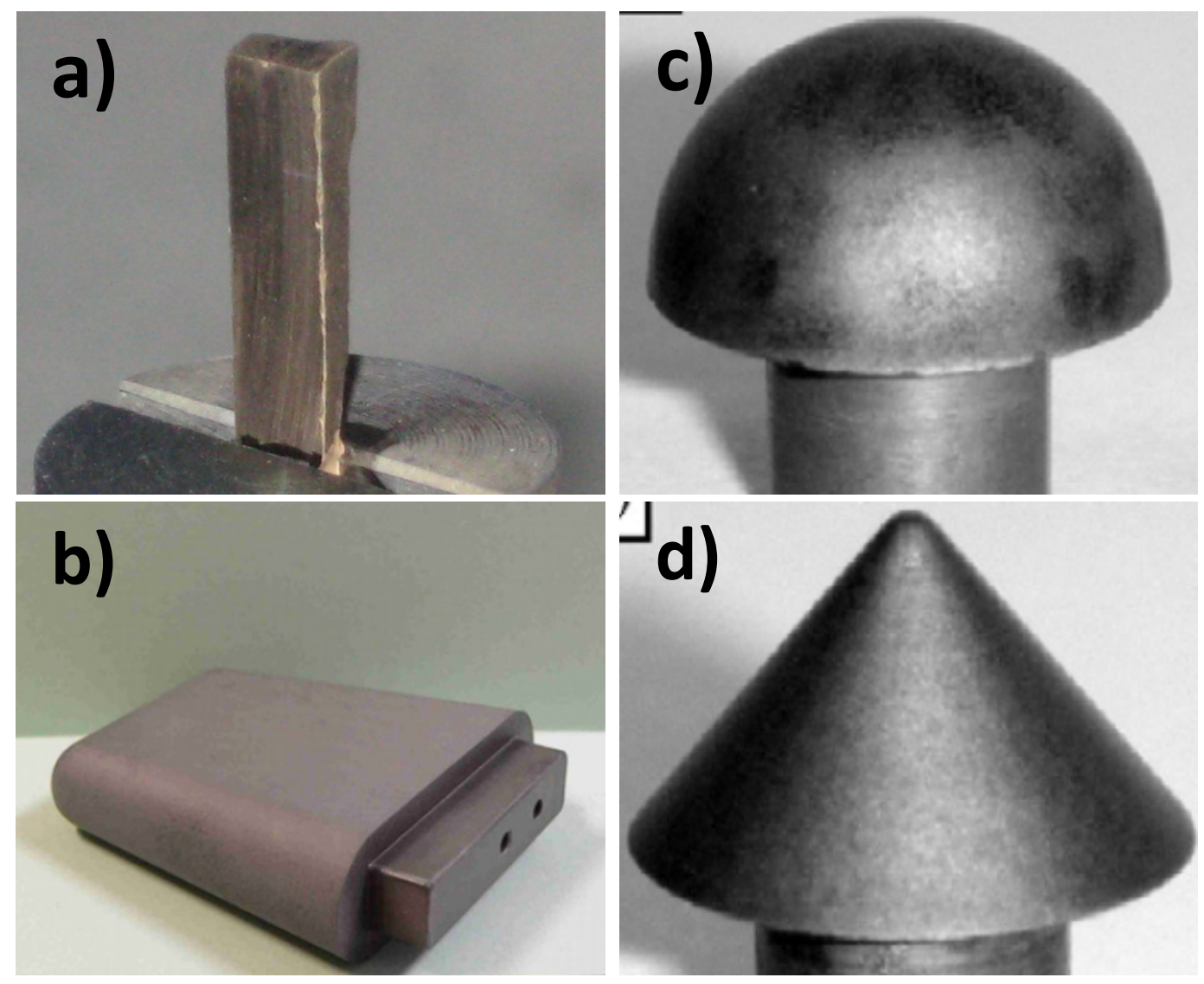

leading edge can be significantly different from that downstream because of differential flow and heat transfer phenomena. Characterization of test environment and oxidation behavior is very challenging in these studies because of the extreme environment and the complicated phenomena involved.

Several aerothermal oxidation studies have been conducted on $\mathrm{ZrB}_{2}-\mathrm{SiC}$ composites under various testing environments and using different model geometries $[135-139,144]$. One of the most important heat transfer mechanisms during aerothermal testing is conduction and therefore a high thermal conductivity is desirable. Monteverde 


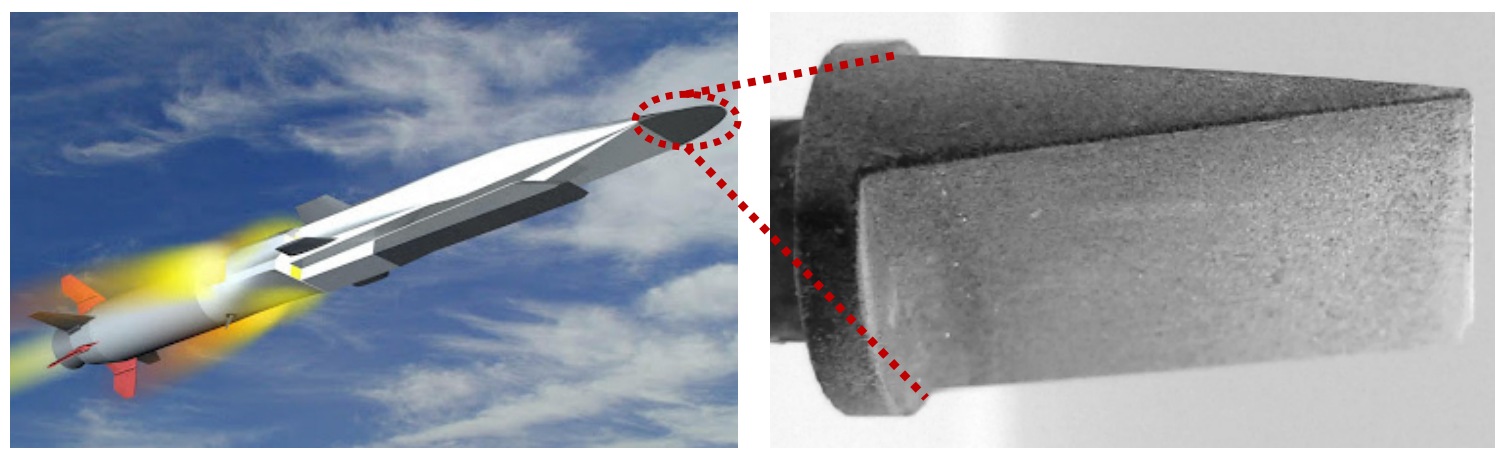

et al [139] elucidate the advantages of UHTCs over lower thermal conductivity ceramics such as $\mathrm{Si} 3 \mathrm{~N} 4-\mathrm{MoSi}_{2}$ by comparing their behavior in a plasma wind tunnel. The samples geometries used for both $\mathrm{Si} 3 \mathrm{~N} 4-\mathrm{MoSi}_{2}$ and $\mathrm{ZrB}_{2}-\mathrm{SiC}$ composites are the same as that shown in Fig 2.14b. The heat fluxes experienced by the models are $7 \mathrm{MW} / \mathrm{m}^{2}$ and this translates to a surface temperature of about $2450{ }^{\circ} \mathrm{C}$ as estimated by computational fluid dynamics (CFD) simulations [139]. The $\mathrm{ZrB}_{2}-\mathrm{SiC}$ material was exposed for 475 seconds at specific enthalpies ranging from $8.1 \mathrm{MJ} / \mathrm{kg}$ to $16.4 \mathrm{MJ} / \mathrm{kg}$, while the $\mathrm{Si} 3 \mathrm{~N} 4-\mathrm{MoSi}_{2}$ material was exposed for 205 seconds at similar enthalpies. Despite the longer exposure time, the $\mathrm{ZrB}_{2}-\mathrm{SiC}$ material significantly outperforms the $\mathrm{Si} 3 \mathrm{~N} 4-\mathrm{MoSi}_{2}$. Fig. 2.15 shows how the $\mathrm{Si} 3 \mathrm{~N} 4-\mathrm{MoSi}_{2}$ material has experienced significant ablation and severe structural deterioration while the UHTC material remains structurally intact. The low thermal conductivity of the $\mathrm{Si} 3 \mathrm{~N} 4-\mathrm{MoSi}_{2}$ material causes heat to accumulate at the point highest exposure and temperatures exceed past the melting point leading to the melting and evaporating of the material. 
The superior oxidation behavior of $\mathrm{ZrB}_{2}-\mathrm{SiC}$ is attributed to its high thermal conductivity as well as the phase transformations that occur during oxidation. The nature and effects of these phase transformations are investigated by characterizing the resulting microstructure after different test conditions. It was found that $\mathrm{ZrB}_{2}-\mathrm{SiC}$ composites undergo minor or no structural deterioration when exposed for 10 min at a heat flux of 1.7 MW $/ \mathrm{m}^{2}$ which generated surface temperatures of $1600{ }^{\circ} \mathrm{C}-1700{ }^{\circ} \mathrm{C}[135]$. When the heat flux was increased to $5.4 \mathrm{MW} / \mathrm{m}^{2}$ the surface temperatures increased to $\sim 2300 \mathrm{C}$ and the leading edge of the sharp UHTC body became ablated. The survival of the UHTC at the lower temperature is attributed to the formation of a multiphase oxide scale. The oxide scale consists of a glassy silica based layer which prevents diffusion of oxygen into the underlying material. Beneath the silica glass there is a 25 um thick layer of zirconia

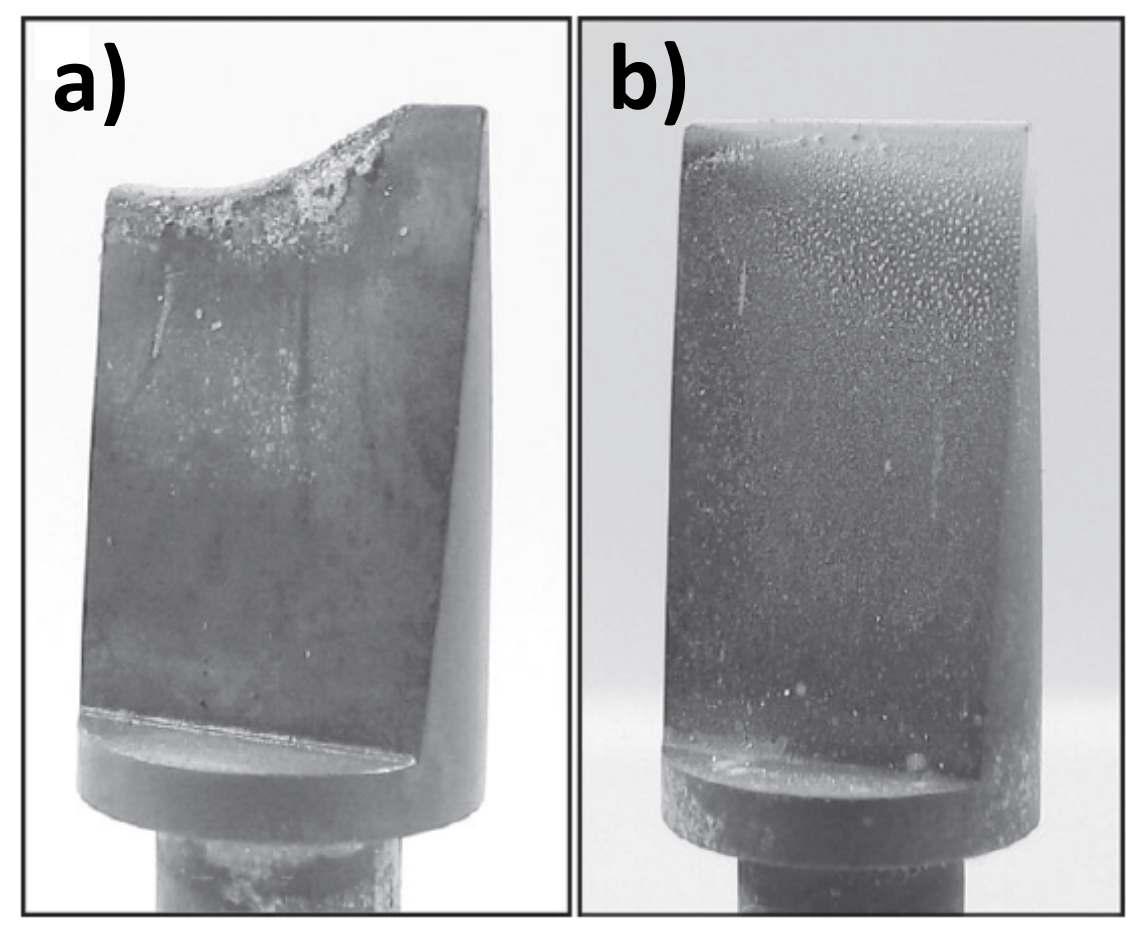


which is believed to strengthen the silicate and keep it from being sheared away by the Mach 3 flow, allowing it to form a continuous silica layer [135]. At higher temperatures, the formed oxide scale softens and is thus more vulnerable to the high shear forces present in the Mach 3 flow which results in the leading edge becoming ablated. The shearing off of silica glass is evident by the large amounts of discontinuous silica glass present on the surface of the samples exposed to $2300{ }^{\circ} \mathrm{C}$.

The microstructural evolution of $\mathrm{ZrB}_{2}-\mathrm{SiC}$ composites has also been observed for experiments done at subsonic speeds [136], atmospheric conditions [137], and longer duration multiple cycle exposures [138]. Alfano et al [136] test $\mathrm{ZrB}_{2}-\mathrm{SiC}$ in a subsonic plasma flow where the temperature is ramping up from $600{ }^{\circ} \mathrm{C}$ to $1550{ }^{\circ} \mathrm{C}$ or from $600{ }^{\circ} \mathrm{C}$ to $1750{ }^{\circ} \mathrm{C}$ in $200{ }^{\circ} \mathrm{C}$ intervals over a period of $60 \mathrm{~min}$. Each hold time lasts $3 \mathrm{~min}$ and the ramp time for each temperature is $4 \mathrm{~min}$. The surface of samples exposed to the $1550{ }^{\circ} \mathrm{C}$ flow consisted of a silica layer while the example exposed to $1750{ }^{\circ} \mathrm{C}$ had a surface of mostly zirconia as the silica layer likely evaporated at this high temperature and sustained exposure time. Monteverde et al [137] expose $\mathrm{ZrB}_{2}-\mathrm{SiC}$ samples to a $1827{ }^{\circ} \mathrm{C}$ plasma flow for 9 minutes. The plasma flow yields specific enthalpies as high as $10 \mathrm{MJ} / \mathrm{kg}$ and is generated using 80 weight percent Nitrogen and 20 weight percent Oxygen in order to simulate atmospheric conditions. Geometries tested included blunt body type and nosecone type, such as those shown in Fig17a and b respectively. Similar behavior was observed for both models and the post oxidation microstructure was similar to that exhibited during other $\mathrm{ZrB}_{2}$-SiC studies. The oxide scale cross section for the nosecone configuration is shown in Fig. 2.16. The outer layer consists of a protective glassy silica 


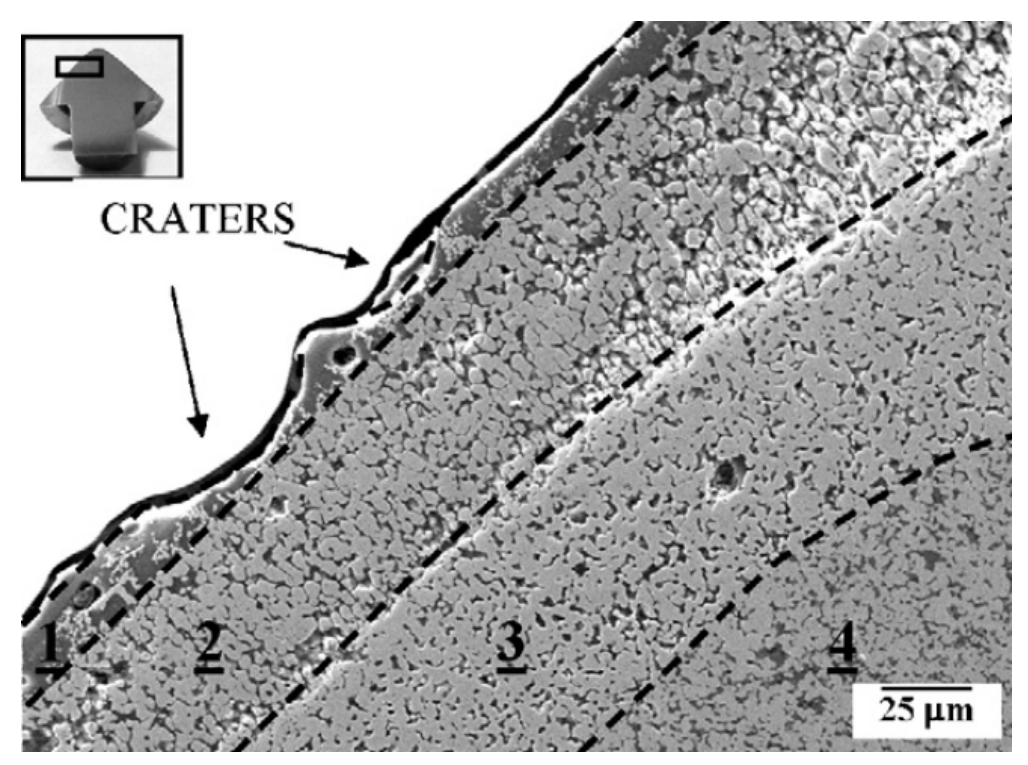

layer, followed by a layer of zirconia embedded in the silica glass. The following layer is a region where $\mathrm{SiC}$ has been depleted due to oxidation and below this layer the unoxidized portion of the cone is found. The craters at the surface of the glassy layer are a signature of gaseous bubble bursting that occurs during oxidation. Sciti et al [138] test $\mathrm{SiC}$ fiber reinforced $\mathrm{ZrB}_{2}$ using four thermal cycles ranging in temperature from $1000{ }^{\circ} \mathrm{C}$ to $1700^{\circ} \mathrm{C}$ for a total of 17 minutes. A sharp edge geometry such as that shown in Fig. 18 is used in this study and the formation of a silica glass become more prominent as the distance from the leading edge increases. Microstructural evolution is consistent with $\mathrm{SiC}$ particle reinforced $\mathrm{ZrB}_{2}$ studies discussed above except that these samples contained residual carbon in the zirconia rich layer.

Aerothermal oxidation tests have been conducted on HfC based UHTCs in plasma flow [140] and oxyacetylene flame [141] environments. Savino et al [140] investigate the 
oxidation behavior of $\mathrm{HfC}-\mathrm{MoSi}_{2}$ in a plasma flow with temperatures up to $2400{ }^{\circ} \mathrm{C}$. The blunt body models are exposed for 100 seconds in one run and for 430 seconds in another run. The addition of $5 \mathrm{MoSi}_{2}$ is designed to induce formation a glassy silica layer as occurs in $\mathrm{ZrB}_{2}-\mathrm{SiC}$ composites. The post-oxidation microstructure consists of a porous outer layer of $\mathrm{HfO} 2$ and an inner layer of $\mathrm{HfO} 2$ and glassy silica. The $\mathrm{HfC}$ oxyacetylene flame study is conducted on a HfC coating on a carbon/carbon composite substrate. The HfC coating is exposed for $60 \mathrm{~s}$ to the oxyacetylene flame whose temperature is determined to be $3000{ }^{\circ} \mathrm{C}$ using an optical pyrometer. $\mathrm{HfC}$ coatings protect the substrate and demonstrate a strong adhesion to the $\mathrm{C} / \mathrm{C}$ substrate. The densest of the $\mathrm{HfC}$ samples tested exhibited the most cracking after oxidation testing and this is attributed to higher thermal stresses [141]. The post oxidation microstructure consists of a porous protective $\mathrm{HfO} 2$ layer as in the previous study. In addition a molten $\mathrm{HfO} 2$ layer forms on top of the porous $\mathrm{HfO} 2$ layer which inhibits oxygen diffusion through the porous $\mathrm{HfO} 2$ layer.

High temperature oxidation tests are conducted on $\mathrm{TaC}$ based composites by $\mathrm{Bao}$ et al [142] in a plasma flame environment for 30 seconds. Flat rectangular shaped samples of $\mathrm{TaC}$, boron nitride particle reinforced $\mathrm{TaC}$ ( $\mathrm{TaC}-\mathrm{BC})$, and carbon nanotubes reinforced $\mathrm{TaC}(\mathrm{TaC}-\mathrm{CNT})$ are investigated. The samples are placed $50 \mathrm{~mm}$ from the plasma gun where the temperature is measured to be $\sim 2575{ }^{\circ} \mathrm{C}$ by two color optical pyrometry. The addition of $\mathrm{BC}$ and $\mathrm{CNT}$ has an adverse effect on the oxidation behavior of $\mathrm{TaC}$. The oxide layer of $\mathrm{TaC}-\mathrm{BC}$ is $1558 \%$ thicker than that of $\mathrm{TaC}$ while the $\mathrm{TaC}$ CNT oxide layer is $42 \%$ thicker than that of TaC. The latter increase in thickness is not very significant especially given the small variations on heat fluxes that may be present 
due to slightly different sample sizes. However, it is also observed that the thermal conductivity of the $\mathrm{TaC}-\mathrm{BC}$ and $\mathrm{TaC}-\mathrm{CNT}$ samples are lower than that of $\mathrm{TaC}$. CNT have a high thermal conductivity and therefore an increase in thermal conductivity would be expected in a TaC-CNT composite. No explanation is given by the authors on why the thermal conductivity would have decreased. The paper shows some promise in that excess carbon provided by $\mathrm{CNT}$ is believed to react with tantalum oxide to form $\mathrm{TaC}$ as explained by Eqn (2.12) and Eqn.(2.13) [142].

$$
\begin{gathered}
2 \mathrm{TaC}+\frac{9}{2} \mathrm{O}_{2} \rightarrow \mathrm{Ta}_{2} \mathrm{O}_{5}+2 \mathrm{CO}_{2} \\
2 \mathrm{Ta}_{2} \mathrm{O}_{5}+7 \mathrm{C} \rightarrow 2 \mathrm{TaC}+5 \mathrm{CO}(\Delta G=1142.3-.823 \mathrm{~T}),\left(\mathrm{T}>1135^{\circ} \mathrm{C}\right)
\end{gathered}
$$

The presence of this reaction is believed to be the reason the oxide layer is similar in thickness to the $\mathrm{TaC}$ oxide despite the lower thermal conductivity.

High temperature aerothermal testing is very important to accessing the performance of UHTC materials in environments similar to those which are they are intended to be used in. Characterizing such extreme environments remains challenging and makes comparison between different experiments difficult. Sample geometry resembling actually aerodynamic components is important to fully accessing the aerothermal phenomena however they introduce other variables such as 3D flow profiles, leading edge curvature, and downstream heat transfer. These additional variables complicate the direct comparison of UHTC microstructures exposed under different testing environments and geometries. 


\section{CHAPTER III: EXPERIMENTAL METHODS}

In this section the experimental methods used to synthesize and characterize the TaC-GNP composites is discussed. The powder processing used to make the composite powders is discussed along with the reasoning behind the method used. The SPS consolidation parameters are presented and discussed. The various microstructural and mechanical characterization techniques are presented along with motives behind employing each technique. Lastly, the experimental setup and characterization methods for conducting oxidation tests in a high temperature plasma flow are discussed.

\section{TaC-GNP Composite Powder Processing}

The starting Tantalum Carbide (TaC) powder is obtained from Inframat Advanced Materials LLC, Connecticut, USA. The Tantalum Carbide has an average particles size of $0.36+/-0.13 \mu \mathrm{m}$. The purity of the Tantalum Carbide powder by weight is $99.7 \%$. The impurities present include carbon $(<0.15 \%)$, oxygen $(0.15-0.30 \%)$, and niobium $(<0.3 \%)$.

Fig. 3.1 below shows SEM images of the as received Tantalum Carbide powder.
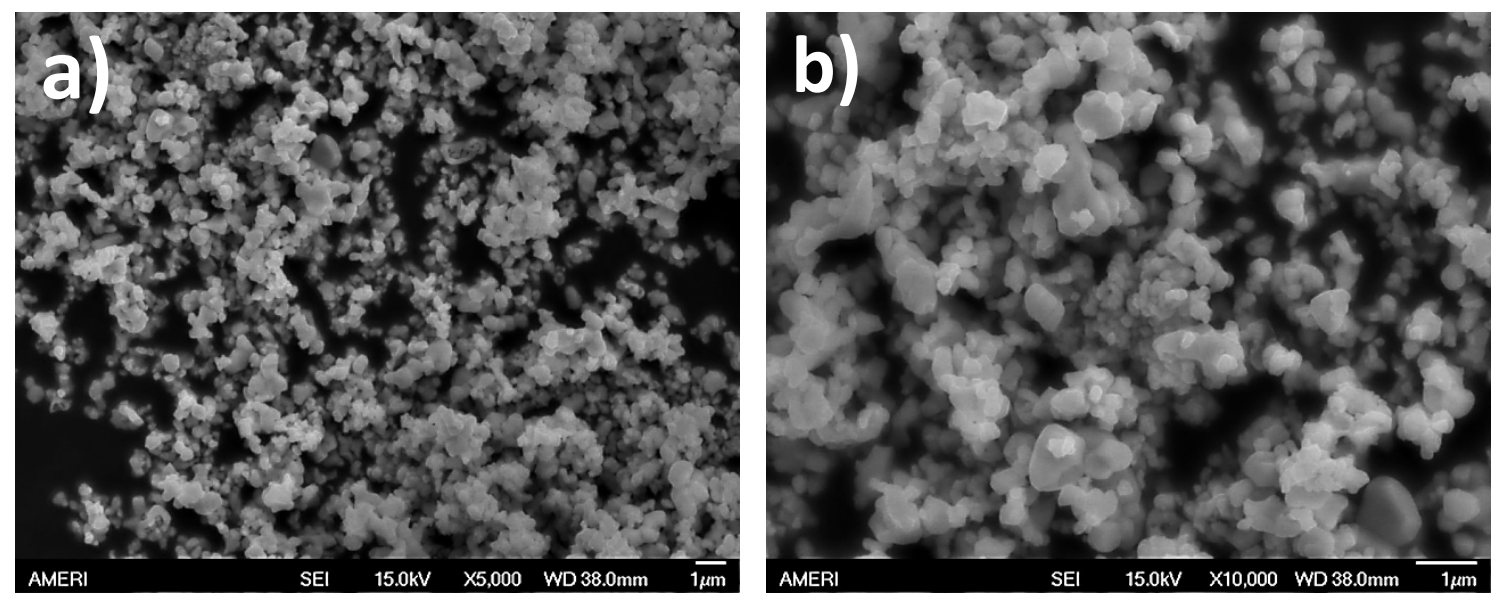
Graphene Nanoplatelets (xGNP-M-5) were obtained from XG Sciences, Lansing, MI, USA. The GNP has a thickness of 6-8 nanometers [27]. This indicates that the average GNP particle has about 20 sheets of graphene as each pair of graphene layers has a thickness of $\sim 0.35 \mathrm{~nm}[81]$. GNP particles have an average diameter of 15 microns, giving the platelets a relative surface area of $120-150 \mathrm{~m} 2 / \mathrm{g}$. [27]. GNPs have some functional groups at the edges, consisting of approximately $0.075 \%$ ether, $0.035 \%$ carboxyl, and $0.03 \%$ hydroxyl groups [27]. Fig. 3.2band c show the SEM and TEM micrographs of the GNPs with wrinkles and folds in the individual GNP. Fig.3.2d shows a folded GNP with the cross-section exposed.. As-received GNPs were used for the synthesis of the bulk GNP structure by SPS to evaluate their survivability in harsh
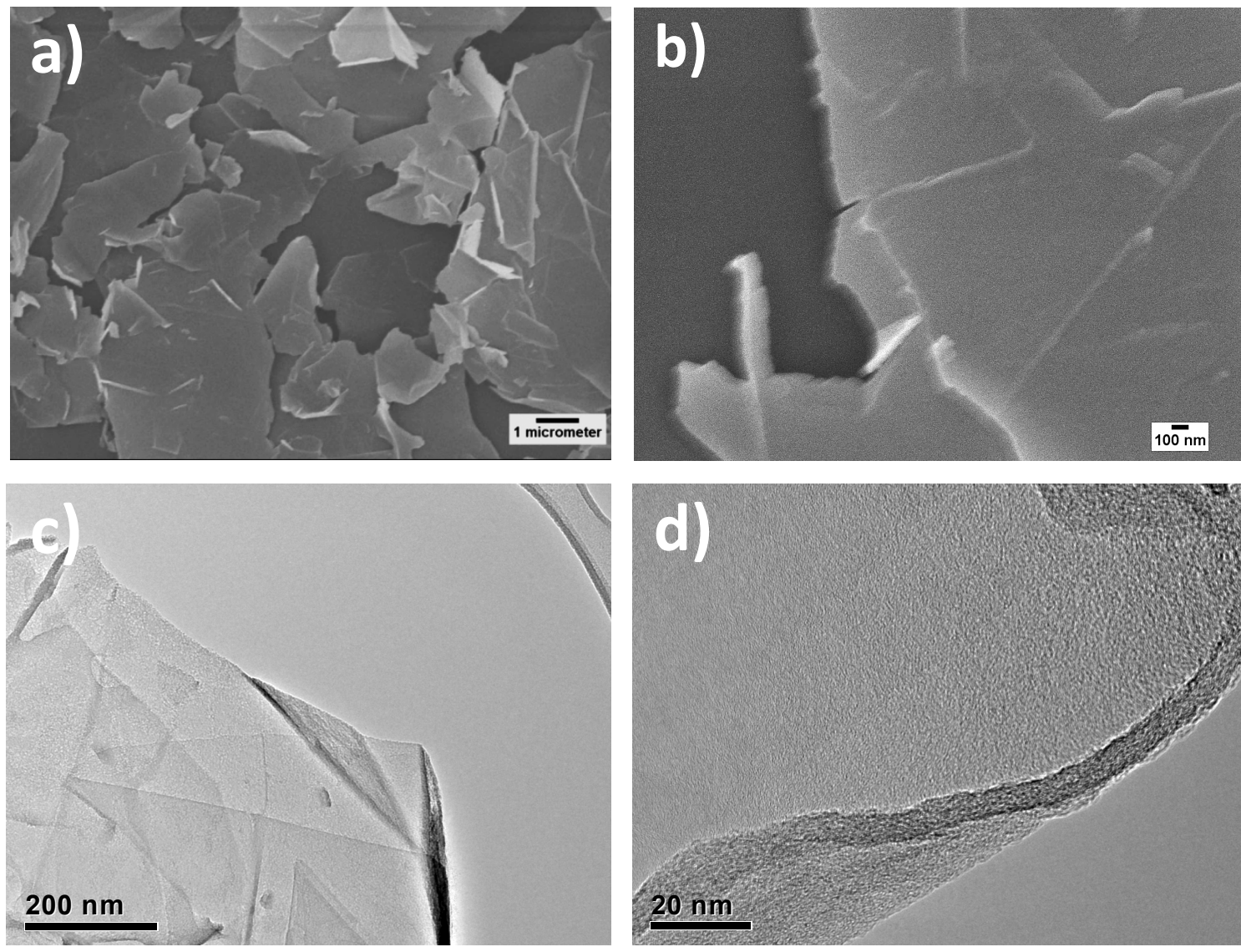
processing conditions. The composite powders are processed from the as received $\mathrm{TaC}$ and GNP starting powders using the powder processing techniques discussed below.

\subsection{Wet Chemistry}

The wet chemistry method was chosen to mix the TaC and GNP powders because of its simplicity and effectiveness. The sonication medium was acetone as no known reactions are believed to occur between acetone and either $\mathrm{TaC}$ or GNP. The compositions to be made ranged from 1 vol. $\%$ to 5 vol. $\%$ of GNP in intervals of 1 vol\%. which yield 5 composite compositions. A pure $\mathrm{TaC}$ composition was also made to serve as a control and so in total 4 powder compositions were produced. The desired amount of powder to be made of each composition was 8 grams; the exact amounts of powders mixed along with the corresponding GNP volume and weight percent is shown in Table

\begin{tabular}{|c|c|c|c|c|c|}
\hline Samples & $\frac{\text { Weight }}{\text { TaC (g) }}$ & $\frac{\text { Weight }}{\text { GNP (g) }}$ & $\begin{array}{l}\text { Weight } \\
\text { Total (g) }\end{array}$ & Weight \% GNP & Volume \% GNP \\
\hline $\mathrm{TaC}$ & 8.00 & 0.00 & 8.00 & 0.00 & 0.00 \\
\hline TaC-GNP-1\% & 7.99 & 0.01 & 8.00 & 0.13 & 1.00 \\
\hline TaC-GNP-2\% & 7.98 & 0.02 & 8.00 & 0.26 & 2.00 \\
\hline TaC-GNP-3\% & 7.97 & 0.03 & 8.00 & 0.39 & 3.00 \\
\hline TaC-GNP-4\% & 7.96 & 0.04 & 8.00 & 0.52 & 4.00 \\
\hline TaC-GNP-5\% & 7.95 & 0.05 & 8.00 & 0.66 & 5.00 \\
\hline GNP & 0.00 & 8.00 & 8.00 & 100.00 & 100.00 \\
\hline
\end{tabular}


3.1. A weight of 8 grams was desired in order to produce $\sim 2 \mathrm{~mm}$ thick samples in a 20 $\mathrm{mm}$ SPS die. The GNP was ultrasonicated first in $400 \mathrm{~mL}$ of acetone in order to remove the agglomerates present in the as-received GNP. The amount of GNP ultrasonicated was determined by the concentration of GNP desired in the composite. After the GNPs were ultrasonicated for $90 \mathrm{~min}, \mathrm{TaC}$ powder was added and mechanically stirred. This TaC-GNP mixture was ultra-sonicated for an additional 90 minutes. The TaC powder used for the control pure $\mathrm{TaC}$ sample was also ultrasonicated for 90 minutes in order to remove agglomerates in as-received $\mathrm{TaC}$ powder.

After the $\mathrm{TaC}$ and GNP powders have been ultrasonicated in acetone, the $\mathrm{TaC}$ GNP-acetone solutions are placed inside an oven for $24 \mathrm{hrs}$. The use of an oven is required as the evaporation rate of the acetone in a vacuum proved to be too slow to be practical. The oven temperature fluctuates between $75-85^{\circ} \mathrm{C}$. This temperature range is chosen as it accelerates the evaporation of the acetone without having any impact on the TaC-GNP powders. After $24 \mathrm{hrs,} \mathrm{the} \mathrm{acetone} \mathrm{has} \mathrm{evaporated} \mathrm{and} \mathrm{the} \mathrm{resulting} \mathrm{powder}$ appears as a continuous shiny layer at the bottom of the beaker as shown in Fig. 3.3. This

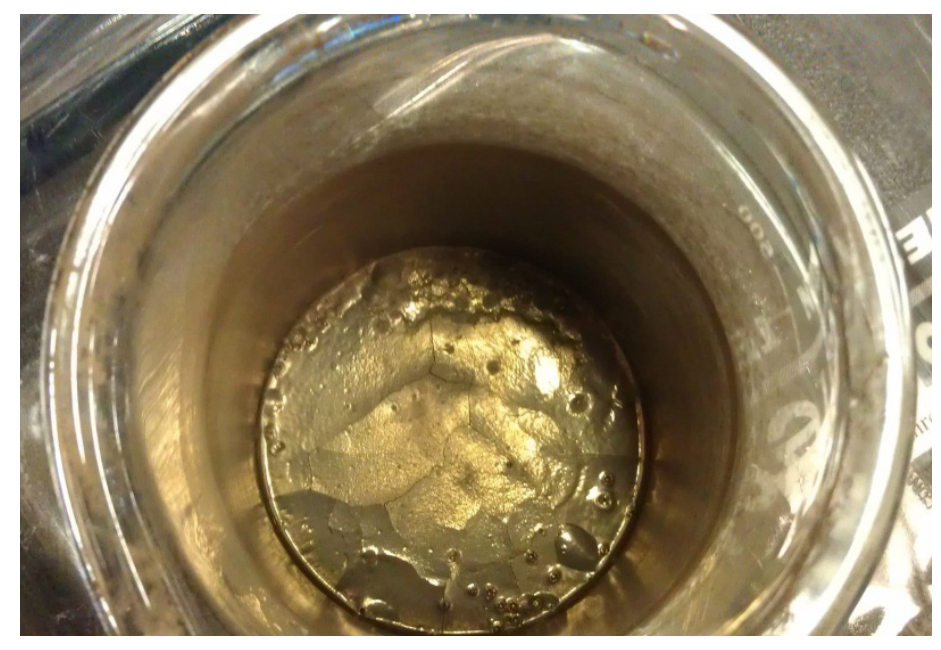


continuous layer must be broken apart in order to allow for complete drying of the powders. The first powder made TaC, was transferred into a large ceramic mortar and crushed with a ceramic pestle. It was found that large amounts of powder remained on the pestle and the mortar walls. This resulted in a significant loss of powder for the $\mathrm{TaC}$ composition. The next powder, TaC-1G, was crushed in a smaller mortar in hopes of reducing powder loss. Powder loss was reduced but still significant. The remaining powders were not transferred to any mortar and instead were crushed inside the beaker with the ceramic pestle. Once the powders were crushed, they were placed in the oven for an additional $12 \mathrm{hrs}$. Crushing and heating process was repeated for an additional $12 \mathrm{hrs}$. Upon completion of this heat treatment the powders were transferred from the beakers to plastic bags.

\subsection{High Energy Ball Milling}

High energy ball milling was utilized to mix another batch of TaC-GNP composite powders. As mentioned in Section 2.2, attrition milling is a widely used method of mixing and refining UHTC powder mixtures. The outcome of the milling is largely affected by parameters such as milling time, ball to powder ratio, milling medium, and ball and jar material to name a few. Compositions of pure $\mathrm{TaC}, \mathrm{TaC}-1 \mathrm{vol} . \% \mathrm{GNP}$, and TaC-5vol\%GNP were prepared by combining the wet chemistry method above followed by high energy ball milling processing. Only these three compositions were made in order to evaluate the potential of the ball milling method before proceeding with the other compositions. 

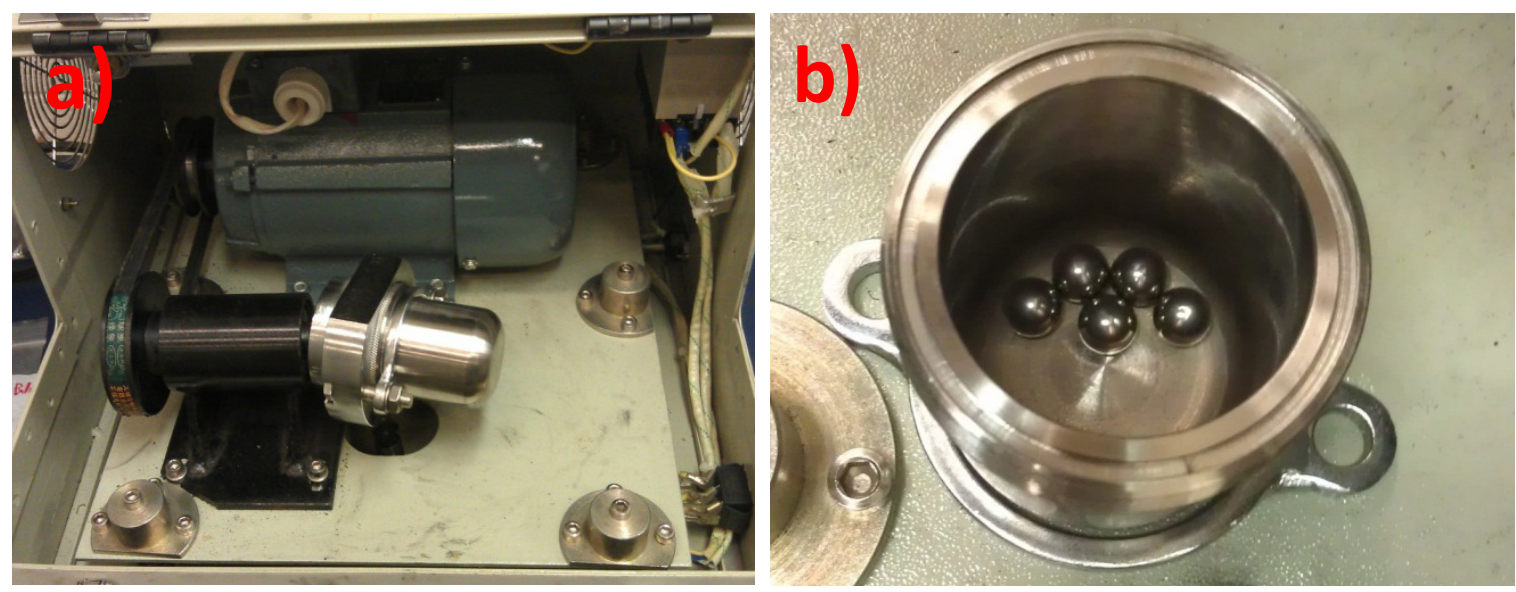

The ball mill used was a WQ-N High Speed High Energy Ball Mill (110V) (Across International, New Jersey,USA) and is shown in Fig. 3.4. The ball mill rotating mechanism oscillates in order to provide a motion in all three dimensions which provides for better mixing. The rotation velocity of this machine is fixed at 1200 RPM. The powders were first mixed by the wet chemistry method explained above and subsequently ball milled for 60 minutes. This is a relatively low time for ball milling and it was chosen in order to minimize damage to the GNPs. The environment inside the ball mill becomes increasingly pressurized and hot as milling time is increased. The kinetic energy of the balls leads to this increase in temperature and pressure in addition to the mechanical crushing and cleaving of the powder. The balls used were made of steel and had a diameter of $10 \mathrm{~mm}$. The ball to powder ratio was .76; the amount of powder milled was 24 grams. More powder was made this time as larger samples were desired and increased powder loss during ball milling was anticipated. The milling jar was also made of steel and only the powders and the steel balls were placed inside the jar during milling (i.e. no vacuum or milling media was used). The powders made by high energy ball 
milling were ultimately not sintered as the SEM and Micro Raman analysis indicated that GNP had been badly damaged during the processing.

\section{Spark Plasma Sintering Processing}

The GNP powder was consolidated at Thermal Technologies LLC (Santa Rosa, California, USA) using a 10-4 spark plasma sintering machine. The consolidation was to be carried out in a $20 \mathrm{~mm}$ diameter graphite die using a holding pressure of $100 \mathrm{MPa}$, a holding temperature of $1850{ }^{\circ} \mathrm{C}$, and a hold time of 10 minutes. The environment in the die consisted of Argon gas at a pressure of $3-6.5 \mathrm{~Pa}$. GNP powder was packed and wrapped in a graphite foil for improved current flow and to prevent reactions between the

powder and the die. The current was held at approximately $2500 \mathrm{~A}$ during the $10 \mathrm{~min}$ hold at $1850{ }^{\circ} \mathrm{C}$. The current at the 3 minute hold at $1100{ }^{\circ} \mathrm{C}$ was approximately $1110 \mathrm{~A}$. The heating rate used was $200{ }^{\circ} \mathrm{C} / \mathrm{min}$.

These temperature and pressure parameters during the 10 minute hold push the limits of the SPS machine and as a result not all samples were sintered at the desired parameters. As explained before, at high temperatures, the strength of graphite decreases making it vulnerable to the high force being applied to reach $100 \mathrm{MPa}$. The use of a silicon carbide die was not used because of the high cost and the fact that in previous studies [69] employing the same parameters a graphite die had been used. The first three powders consolidated were the TaC-1GNP, TaC-2GNP, and TaC-3GNP powders and as can be seen from Fig. 3.5a-c this powders were consolidated at the desired pressure 

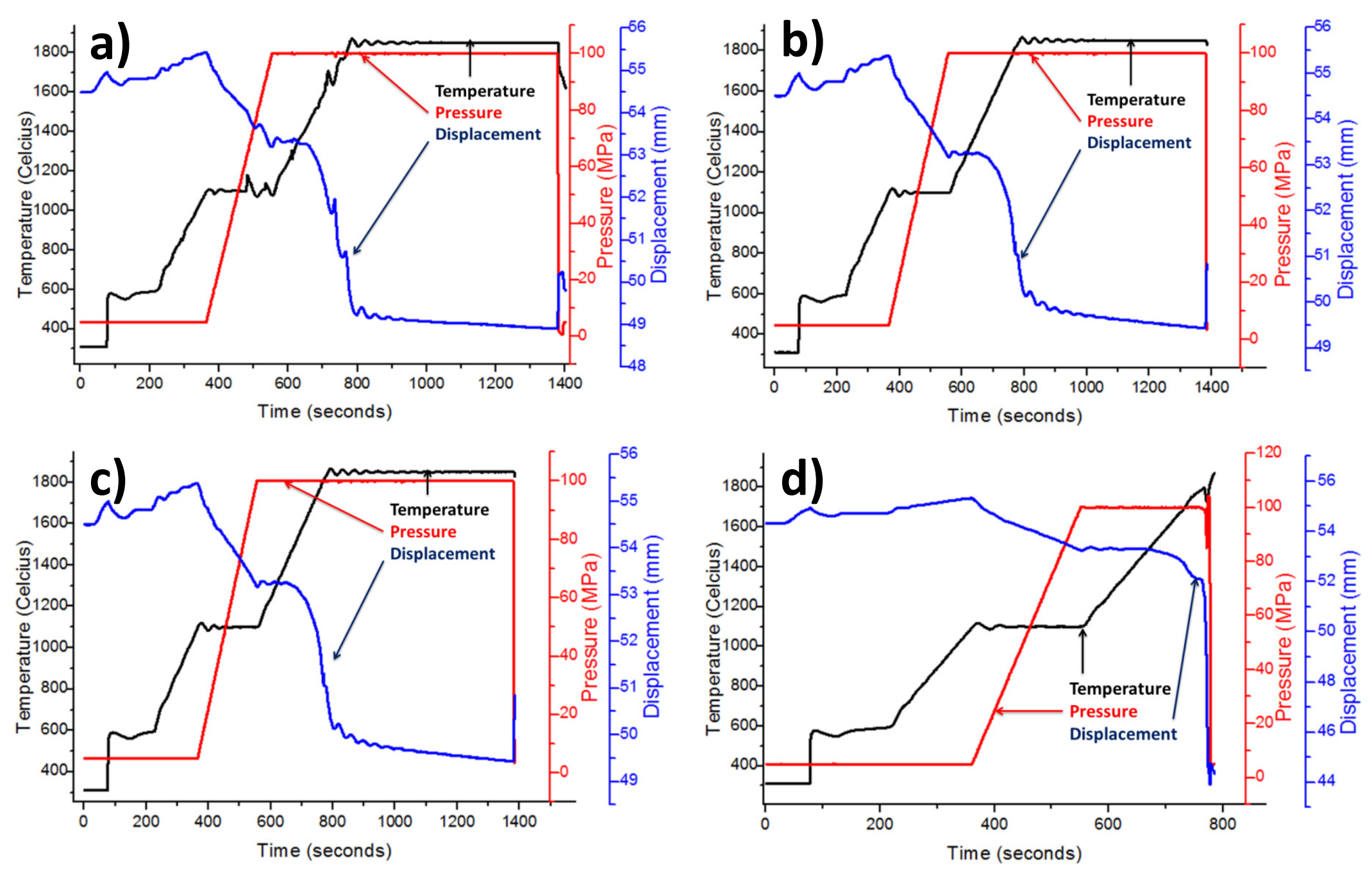

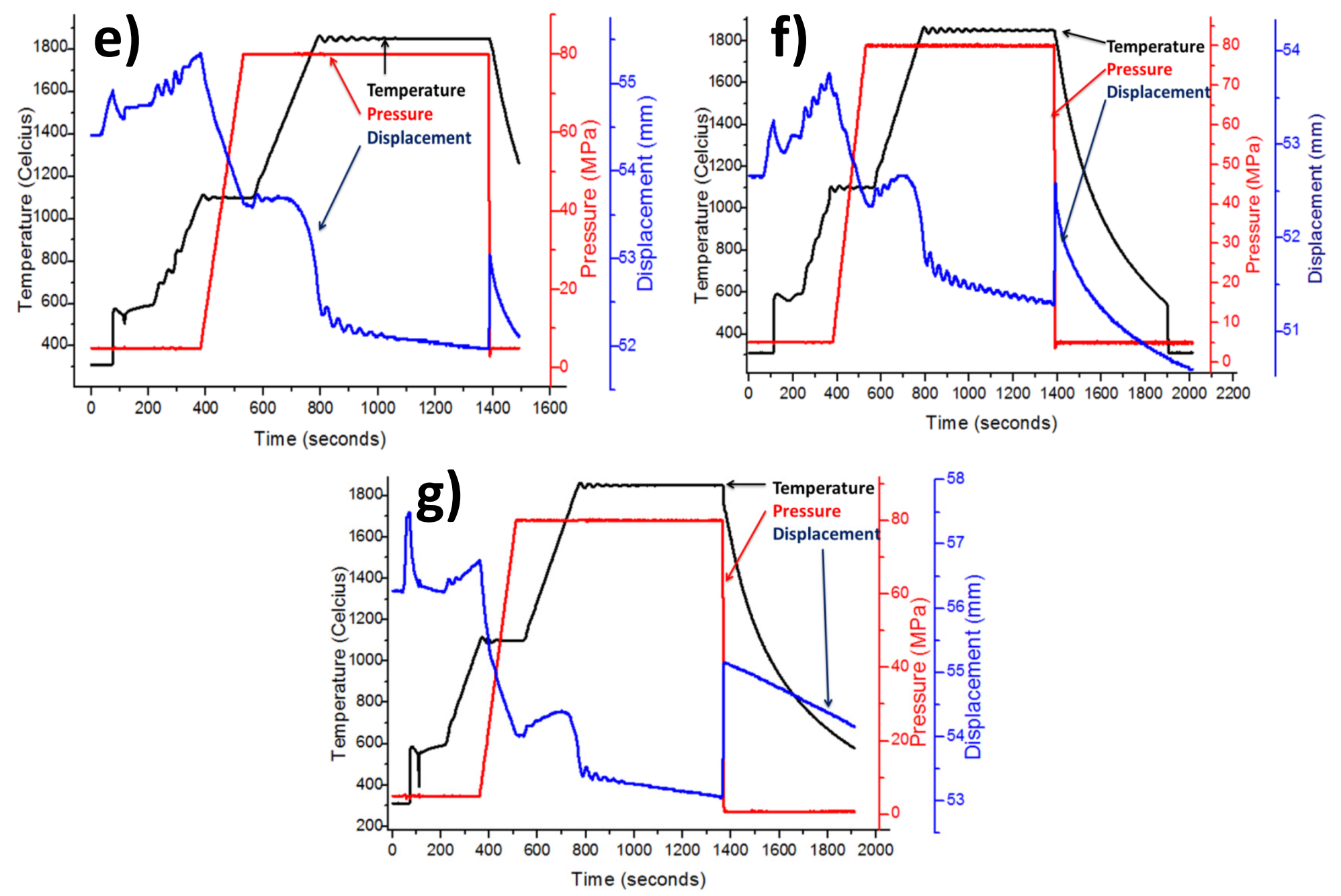
(100MPa), temperature $\left(1850^{\circ} \mathrm{C}\right)$, and hold time $(10 \mathrm{~min})$. The fourth powder to be made was the $\mathrm{TaC}-4 \mathrm{G}$ sample and as can be seen from Fig. $3.5 \mathrm{~d}$, the pressure drops sharply shortly after reaching $1850{ }^{\circ} \mathrm{C}$ and so the powder is held under the desired pressure and temperature for $\sim 1$ minute. This drop in pressure is due to the failure of the graphite die and the subsequent shut down of the SPS. To avoid the risk of destroying the available graphite dies which would prevent the remaining samples from being made; the holding pressure was lowered to $80 \mathrm{MPa}$ for the remaining samples, which were the TaC-5GNP, pure TaC, and pure GNP samples. These three samples are successfully sintered at 80 $\mathrm{MPa}, 1850^{\circ} \mathrm{C}$, and a holding time of 10 minutes as can be seen from Fig.3.5e-g.

\section{Structural Characterization}

The bulk GNP, TaC, and TaC-GNP pellets obtained through SPS processing were polished in order to remove the graphite foil from the surface. This thin layer of graphite originates from the graphite foil used during the SPS to keep the powder from contacting the die. A Helium gas pycnometer (Accupyc 1340, Micrometrics Instrument Corporation, Norcross, GA, USA) was used to measure the true density of the bulk pellets as well as the starting powders. A Buehler Versamet 3 Optical Microscope (Illinois, USA) is used to observe the polished cross sections of the sintered compacts. Optical microscopy is used to obtain a visual assessment of the pellet's densification. In order to determine whether any phase transformations have been induced by the SPS processing, X-ray diffraction experiments are carried out. X-ray diffraction (XRD) experiments were carried out on the starting powders and the bulk pellets using a Siemens D-500 X-ray 
diffractometer. The operating voltage and current are $40 \mathrm{kV}$ and $40 \mathrm{~mA}$ respectively. The radiation used is $\mathrm{Cu} \mathrm{K} \alpha$ which has a wavelength of $1.542 \AA$. The starting powders and the bulk pellets were also characterized using Micro-Raman spectroscopy in order to study the effects of the SPS processing on the GNP structure. The equipment used was a Spectra Physics (Model 3900S, California, USA) with Ti-sapphire crystal as the target $(514 \mathrm{~nm})$, a laser power of $18 \mathrm{~mW}$ and a detector with $4 \mathrm{~cm}-1$ spectral resolution from Kaiser Optical Systems, Inc. (Michigan, USA). Scanning electron microscopy of the powders and the bulk pellets were done using a JEOL JSM-633OF field emission scanning electron microscope (FE-SEM) with an operating voltage of $15 \mathrm{kV}$. Image $\mathrm{J}$ software is used analyze the SEM micrographs of the $\mathrm{TaC}$ and $\mathrm{TaC}$-composite samples in order to measure the grain sizes.

\section{Mechanical Properties Characterization}

The sintered compacts were fractured with a hammer and mounted in an epoxy resin in order to study the microstructures and evaluate mechanical properties. For the Bulk GNP sample both the top and transverse surfaces were mounted and polished. The bulk GNP transverse and top surfaces were grinded using 600 grit silicon carbide paper followed by polishing using an alumina slurry of 0.3 micrometer particles. The $\mathrm{TaC}$ and TaC-GNP composite compacts were grinded using progressively finer silicon carbide papers (120, 240, 400, 600 grit size), and then polished using diamond slurries with 1.0 micrometer particles and .10 micrometer particles. In order to evaluate mechanical 
properties at multiple length scales, hardness testing was conducted using microindentation and nanoindentation.

\subsection{Microhardness}

\subsubsection{Low Load Testing}

The microhardness is measured using a microhardness tester with a Vickers tip (Shanghai Taiming Optical Instrument Co. Ltd., model HXD-1000 TMC, Shanghai). The microhardness of both the transverse and top surfaces of the bulk GNP sample is measured; a load of 25 grams $(\sim .25 \mathrm{~N})$ was utilized, with the dwell time being 15 seconds. The TaC and TaC-GNP samples were tested using a load of 500 grams $(\sim 5 \mathrm{~N})$, with the dwell time being 15 seconds. Ten indentations were done at this load for each of the TaC and TaC-GNP samples.

\subsubsection{High Load Testing}

In order to evaluate the materials performance under loads more representative of the structural applications for which they are intended for, TaC and TaC-GNP composites were subject to high load microindentations. TaC, TaC-1G, and TaC-5G samples were sent to Bruker Tribology and Mechanical Testing (Campbell, CA, USA) for higher load microhardness testing and the desired loads exceeded the capabilities of the machine used for $5 \mathrm{~N}$ load tests. A Vickers tip was used as before and the indentation time was 15 seconds as before. Microindentations were performed using loads of $20 \mathrm{~N}$ and $30 \mathrm{~N}$, five tests at each load were performed on each of the samples. 
The samples were then sent to Bruker Stylus and Optical Metrology (Tucson, AZ, USA) for observation of the higher load indents by optical profilometry. The optical profilometry provided data on the amount of volume deformed during the indentations as well as presence of large cracking induced by indentations. The indentations were subsequently observed using SEM and the lengths of the generated cracks was measured using Image J software. The crack length (c) was measured from the center of the indent and used to calculate the fracture toughness $\left(\mathrm{K}_{\mathrm{IC}}\right)$ through use of the Anstis equation [145], Eqn. (3.1).

$$
K_{I C}=.016\left(\frac{E}{H}\right)^{\frac{1}{2}}\left(\frac{P}{c^{\frac{3}{2}}}\right)
$$

The other parameters needed to use the Anstis equation are the load (P) which is either 20 $\mathrm{N}$ or $30 \mathrm{~N}$ depending on which indent generated the crack being measured. The hardness value $(\mathrm{H})$ is known from the hardness tests used to generate the cracks. The elastic modulus (E) values are obtained from the nanoindentation experiments conducted, which are explained in detail in the next section.

\subsection{Nano-Indentation}

Nano-indentation experiments were carried out using a Hysitron Triboindenter (Hysitron Inc., Minneapolis, MN, USA) with a $100 \mathrm{~nm}$ Berkovich tip. The tip-area calibration was done using a standard fused quartz substrate of known modulus (69.6 GPa). The triboindenter was used in quasi-static indentation mode in order to measure the 
elastic modulus and hardness of the sintered compacts. Nanoindentations were performed on both the transverse and top surfaces of the GNP pellet. The indentation process consisted of a 10 second period to reach a load of $125 \mu \mathrm{N}$, with a holding time of 3 seconds, followed by a 10 second unloading to $0 \mathrm{~N}$. For the $\mathrm{TaC}$ and TaC-GNP composite samples the indentations were done on a polished cross section of the material. The indentation process for these very hard samples consisted of a 10 second period to reach $4500 \mu \mathrm{N}$, with a holding time of 3 seconds, followed by a 10 second unloading to 0 N. The elastic modulus was calculated from the load-displacement unloading curves using Oliver-Pharr method [146].

\section{TAC-GNP Oxidation Behavior}

TaC-GNP oxidation studies were conducted in a high temperature plasma flow in order to more closely simulate the conditions experienced during hypersonic flight which is the intended application of these materials. As discussed in Section 2.5, the extreme conditions present in aerothermal testing makes characterization of the oxidation environment as well as the oxidized samples very challenging. The experimental setup, plasma flow parameter controls and measurement techniques, and oxide characterization methods are discussed in this section.

\subsection{Plasma Jet Testing}

The TaC-GNP oxidation test samples are cut out from the consolidated SPS compacts as shown in Fig. 3.6 in order to be accommodated and secured onto the fixture 


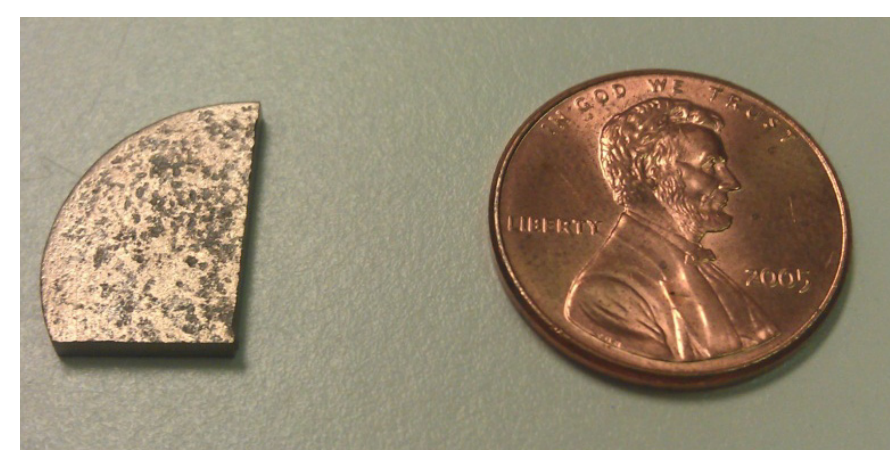

which was originally designed for small rectangular samples. A specimen holder which could withstand the high temperature plasma flow was needed and so the tungsten fixture shown in Fig. 3.7 is used to hold the TaC-GNP samples. The fixture has a $1 \mathrm{~mm}$ diameter hole machined in the backside in order to accommodate a thermocouple to measure the backside temperature of the samples. The fixture is secured in an axial vise in order to prevent excessive vibrations due to the high speed plasma flow. The backside

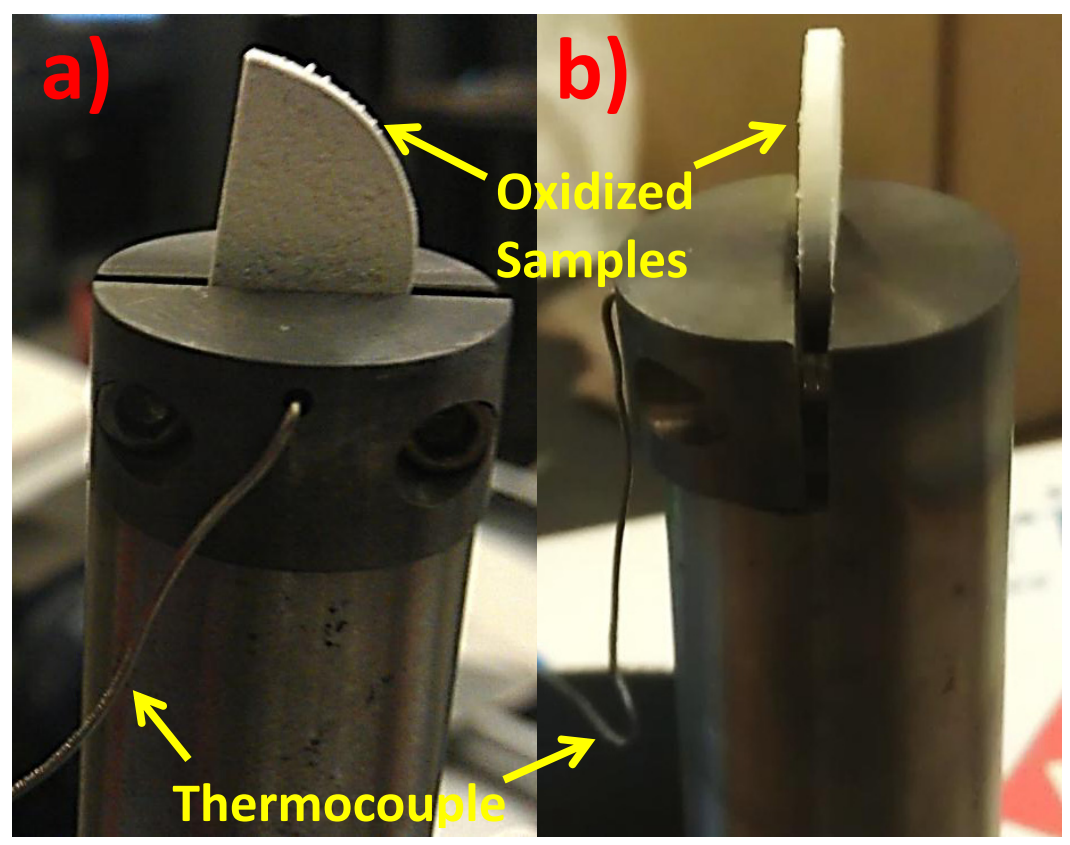




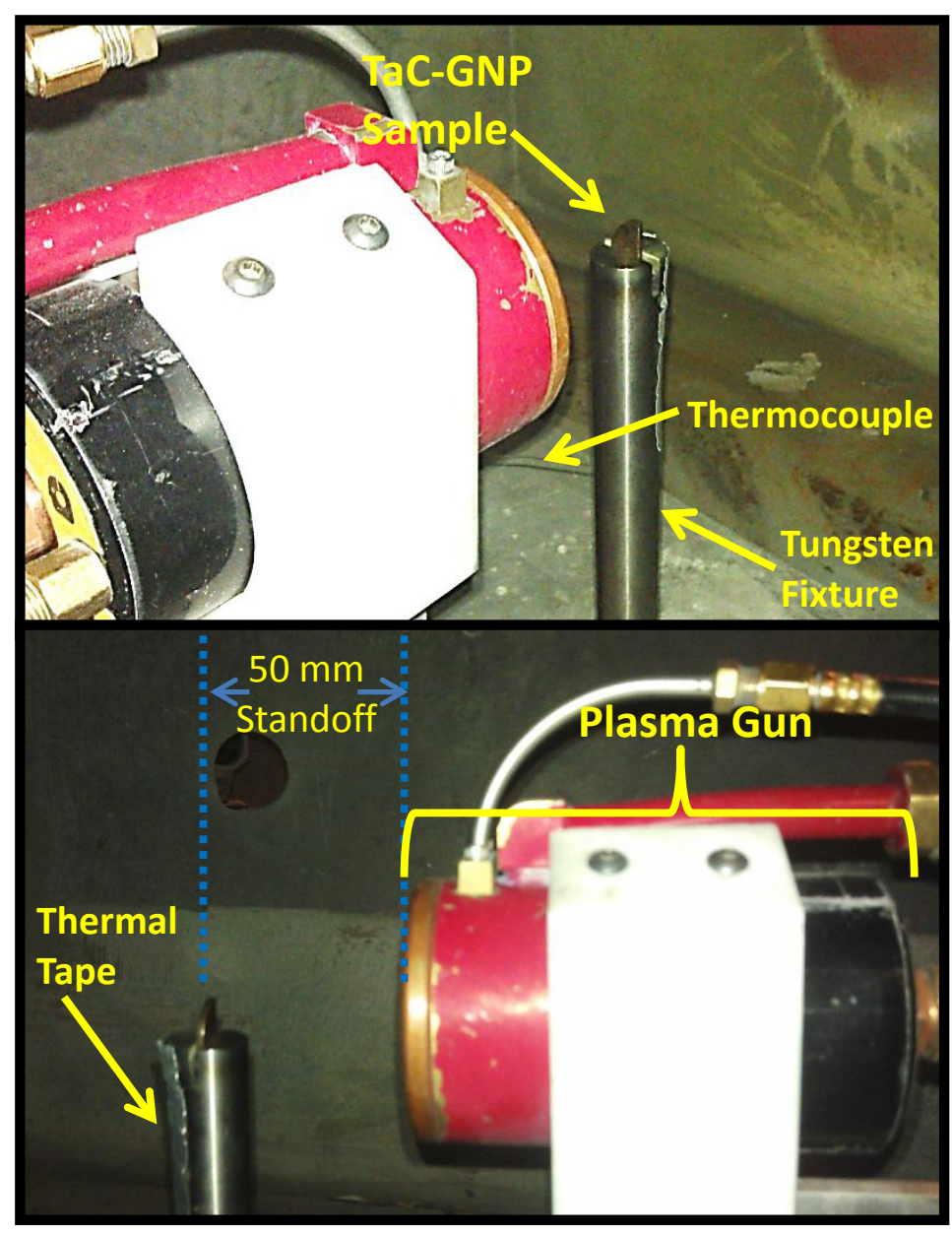

temperature is needed in order to estimate the thermal gradient experienced by the sample during exposure to the plasma flow. Front side temperature cannot be measured by a thermocouple as the high temperature plasma flow will damage and/or destroy the thermocouple. A K-type thermocouple (KMQSS-020U, Omega Engineering Inc.) is inserted into the hole and then bent 90 degrees up in order to ensure adequate contact with the sample. The front side temperature cannot be accurately measured directly so instead an Accuraspray in-flight diagnostic sensor (Tecnar Automation Ltee, QC, Canada) is used to measure the plasma temperature at the point where the front of the sample would be located. This sensor is typically used to measure the temperature of 
powder particles during thermal plasma spraying. However, it serves the purpose of providing a rough estimate of the temperature of the flow at the point of contact with the TaC-GNP sample. The sensor utilizes two color pyrometry (147) to make temperature measurements and a high speed camera to make velocity measurements. The flow is found to have a temperature of $2575+/-50{ }^{\circ} \mathrm{C}$ at the operating plasma parameters described below.

The plasma flow is generated using a Praxair SG 100 Direct Current plasma spray gun (Danbury, CT, USA). The plasma is formed with argon as the primary gas and helium as the secondary gas, the plasma power is set at $32 \mathrm{~kW}$. The argon primary gas flows at a rate of 56 standard liters per minute (slpm) and the helium secondary gas flows at a rate of $60 \mathrm{slpm}$. The configuration of the plasma gun equipment and the sample holder is shown in Fig. 3.8. The distance from the front of the plasma gun to the front surface of the TaC-GNP composite samples is $50 \mathrm{~mm}$. The TaC-GNP samples are exposed to the plasma flow for 60 seconds.

\subsection{Characterization of the Oxide}

The surface of the sample to be oxidized is characterized prior to oxidation using a JEOL JSM-633OF field emission scanning electron microscope (FE-SEM) with an operating voltage of $15 \mathrm{kV}$ and a Nanovea PS50 Optical Profilometer (Irvine, CA, USA). This analysis is done in order to characterize the initial surface morphology and roughness $(\mathrm{Ra})$ of the samples. After the 60 seconds high temperature plasma exposure 
the top surface is again evaluated using FE-SEM and optical metrology. This allows for the effects of oxidation on the sample morphology to be examined. The top surface of the oxidized sample also undergoes X-Ray diffraction (XRD) analysis using a Siemens D500 X-ray diffractometer. This XRD analysis is compared with that done on the SPS compacts in order to identify and quantify phase changes after the 60 second exposure to the plasma flow.

The oxidized samples are then cut and the cross section is polished in the same manner as the SPS pellet cross sections. The cross sections are examined with a Buehler Versamet 3 Optical Microscope (Illinois, USA) in order to measure the oxide thickness and surface recession of the samples. FE-SEM is done on the oxide cross sections and oxidized top surfaces to look into the microstructural evolution of the oxide scales. The change in composition of the oxide scales is quantified by performing an energy dispersive X-Ray spectroscopy (EDS) analysis on the cross section of the oxides. A JEOL JSM 5900LV SEM equipped with an EDAX EDS system is used to conduct the EDS analysis. Microhardness tests using a microhardness tester with a Vickers tip (Shanghai Taiming Optical Instrument Co. Ltd., model HXD-1000 TMC, Shanghai) are conducted in order to quantify the deterioration in strength of the oxidized portion of the TaC-GNP composites. A load of 100 grams $(\sim 1 \mathrm{~N})$ is used, with the holding time remaining 15 seconds as before. 


\section{CHAPTER IV: RESULTS AND ANALYSIS}

This chapter presents experimental results along with an analysis and discussion of the underlying mechanisms at play. The results begin with the characterization of the starting powders in terms of dispersion. Structure retention and behavior of bulk GNP sample is investigated in order to determine feasibility of spark plasma sintering GNP powders at high pressure and temperature, and also to gain insight on potential toughening mechanisms. Processing and microstructure of TaC-GNP samples is presented with a focus on the effects and structure of GNP. Mechanical properties are then discussed along with the relevant GNP induced toughening mechanisms. Lastly, results of high temperature plasma jet oxidation tests are discussed.

\section{TaC-GNP Powder Characterization}

As was previously discussed in Chapter 3, the results of the $\mathrm{TaC}-2 \mathrm{G}$ and $\mathrm{TaC}-4 \mathrm{G}$ samples are not a focus of this study and hence the results are omitted except where relevant. The TaC-2G sample was omitted for its similarity in composition and structure to the $\mathrm{TaC}-1 \mathrm{G}$ and $\mathrm{TaC}-4 \mathrm{G}$ sample was omitted for the variation in processing parameters.

\subsection{Homogeneity and Dispersion of GNP}

Uniform dispersion and homogeneity of starting powders is crucial to ensuring an effective composite material. The reinforcement phase, GNP, must be dispersed uniformly into the $\mathrm{TaC}$ matrix in order to provide adequate interactions between the 


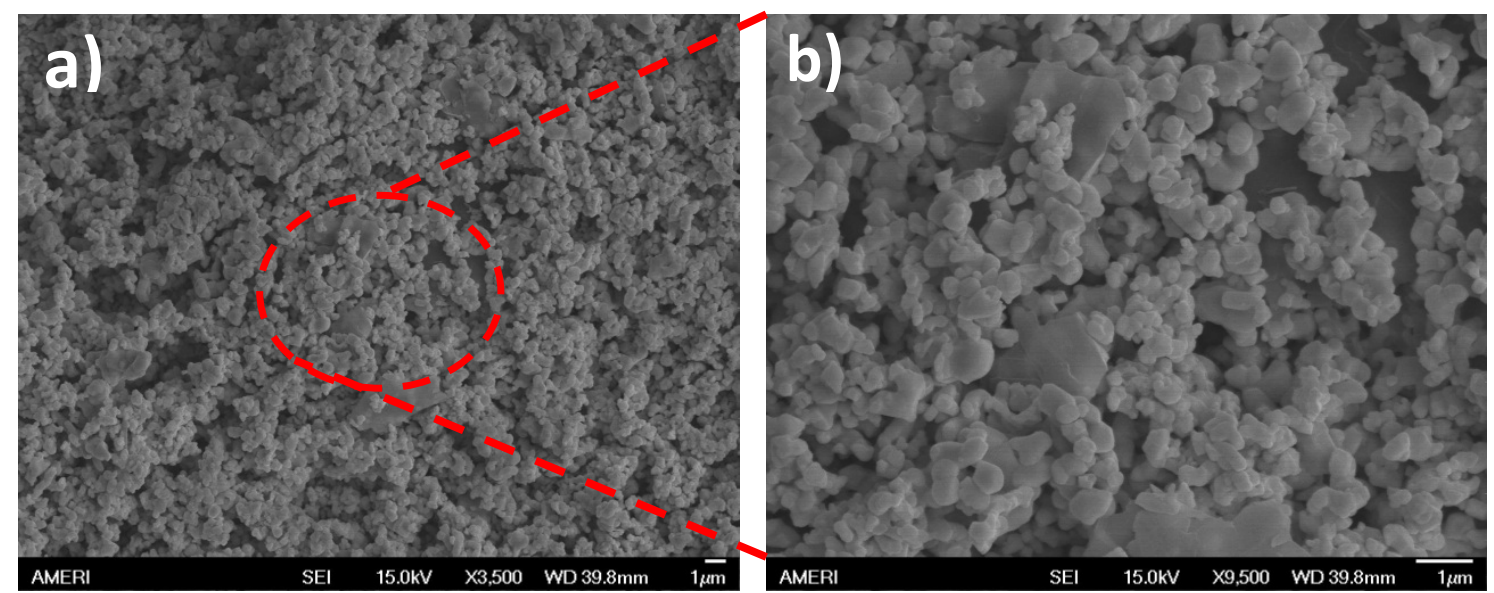

Fig. 4.1: TaC-1G Powder processed by Wet Chemistry a) Low Mag, b) High Mag

matrix and reinforcement phases during sintering. Homogeneous distribution of secondary phases is vital to ensuring uniform properties.

\subsubsection{Powder Processed by Wet Chemistry}

The TaC-GNP powders processed by wet chemistry were examined using scanning electron microscopy (SEM) in order to determine the degree of GNP dispersion and the homogeneity of the powder. Fig. 4.1 and Fig. 4.2 show SEM micrographs of the TaC-1G powders after being processed by wet chemistry. The TaC-1G powder in Fig. 4.1

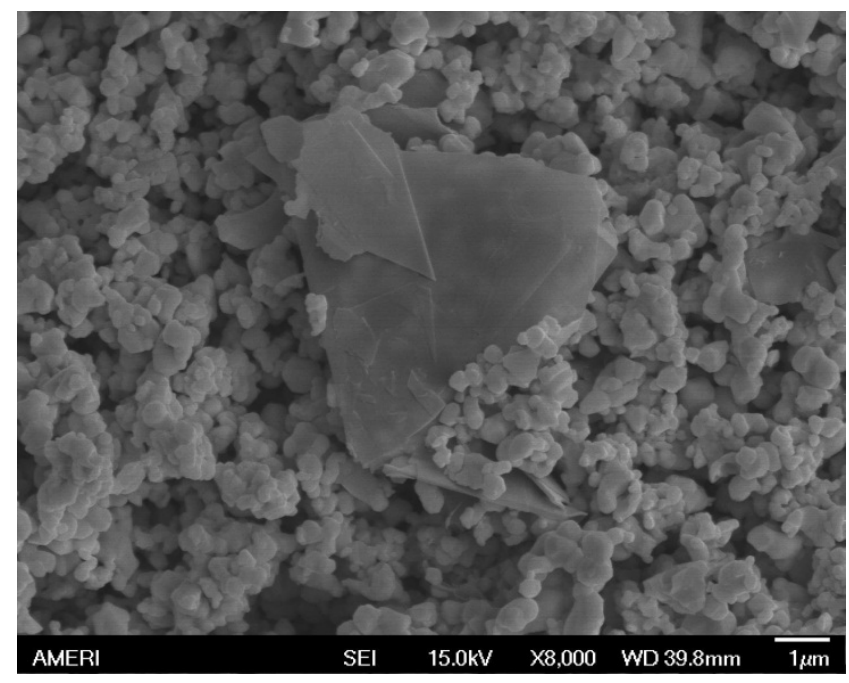




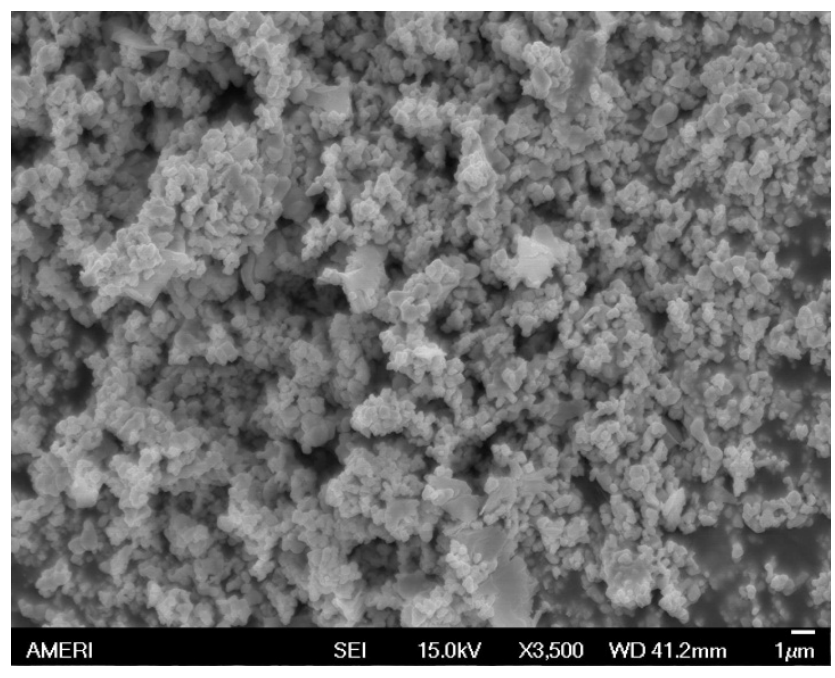

is homogenous in nature with uniform GNP dispersion. As shown in Fig. 4.2, the GNP exhibits a degree of transparency as one can clearly see the $\mathrm{TaC}$ particles lying beneath the platelet. This degree of transparency indicates that GNP platelet is thin and contains fewer sheets of graphene than the other more opaque platelets.

The increased GNP content in the TaC-3G powder can be seen clearly from the SEM micrographs of the TaC-3G powder shown in Fig. 4.3 and Fig. 4.4. As with the TaC-1G powder, the TaC-3G powder shows a large degree of homogeneity. The GNP
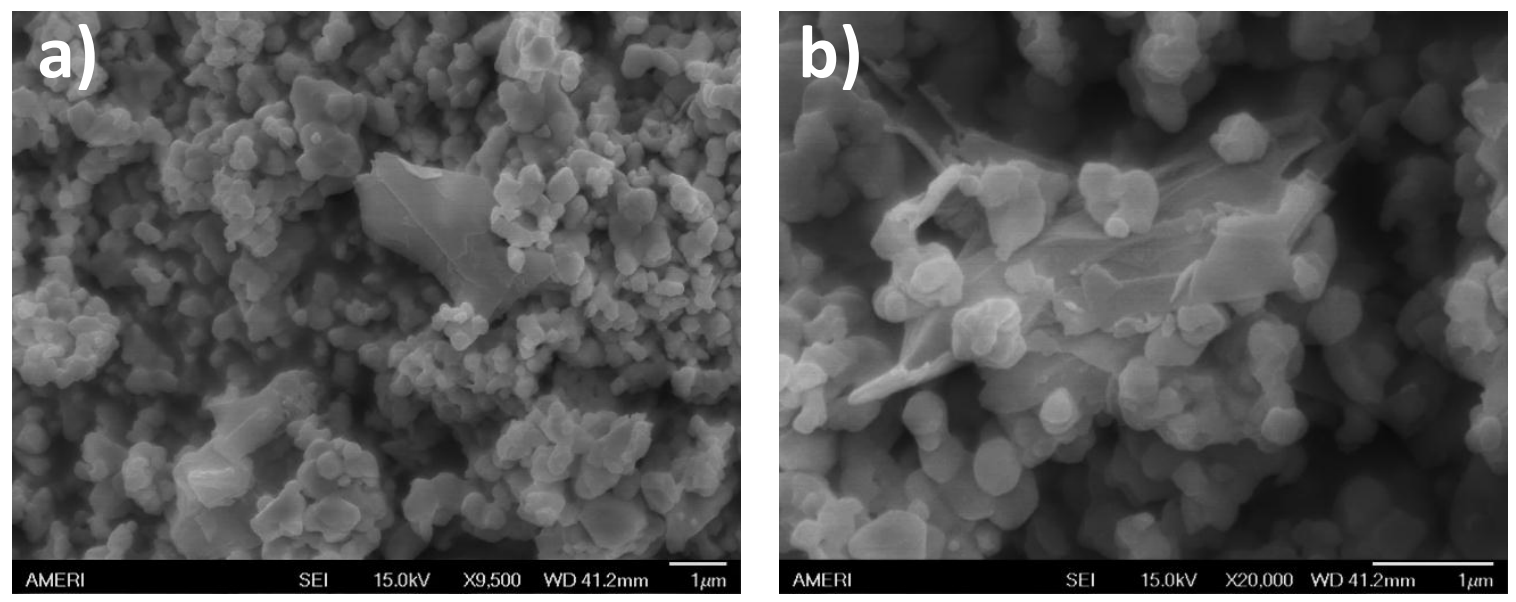


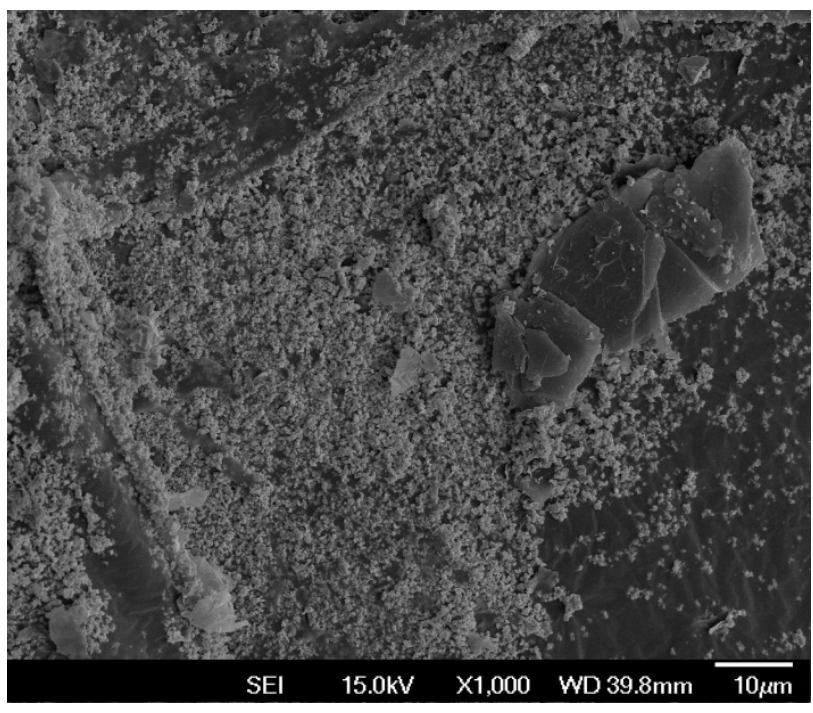

particles appear well distributed in Fig. 4.3. Submicron TaC particles tend to decorate and become clumped around the platelets. Fig. 4.4 shows the diversity of GNP particles present; a transparent GNP is seen in Fig. 4.4a, and in Fig. 4.4b a heavily wrinkled and folded GNP is shown. The wrinkles and folds in the GNP may allow for TaC particles to become entrapped or sandwiched leading to enhanced mixing. The mixing of the GNP and $\mathrm{TaC}$ particles appears to be superior in the $\mathrm{TaC}-3 \mathrm{G}$ powder as compared to the $\mathrm{TaC}$ $1 \mathrm{G}$ powder.

The powder with the highest GNP concentration was the TaC-5G sample. The increased GNP volume appears to have an adverse effect on the dispersion of GNP in the TaC matrix. Fig. 4.5 shows several very large GNPs (30-40 $\mu \mathrm{m}$ wide) segregated from the TaC powder particles. These large GNP have obviously not been adequately mixed with the TaC powder and may have undesired effects on the microstructure of the sintered compacts. Smaller GNP particles are shown in Fig. 4.6; they are not segregated from the TaC powders as with the larger GNPs, however the mixing is still poor as there 


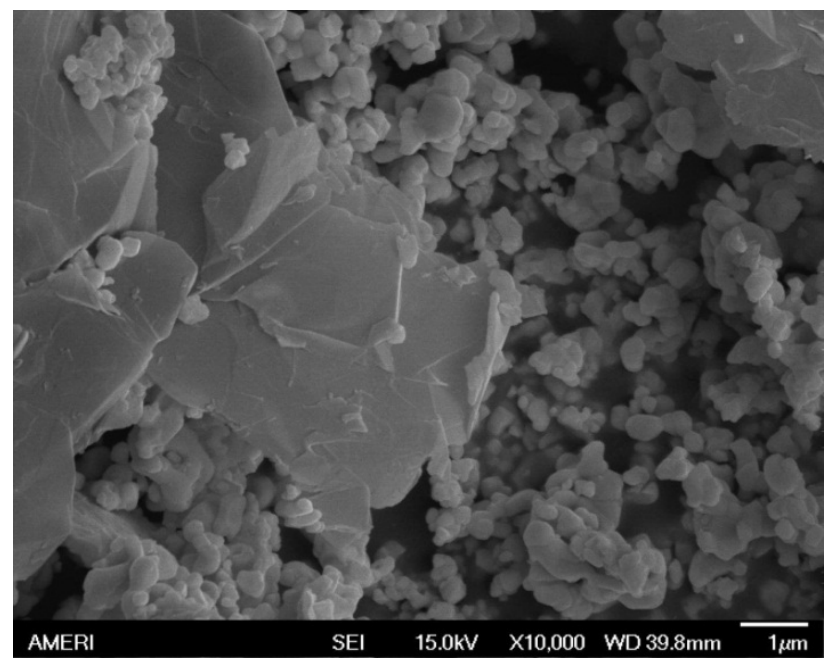

are few TaC particles lying on the surface of the GNPs. The varying types of GNP can be seen in Fig. 4.7; an agglomerated ball of GNP can be seen alongside smooth transparent GNP. A few regions in the TaC-5G powder, such as that shown in Fig. 4.8, exhibit better GNP dispersion and powder homogeneity. These small transparent GNPs shown in Fig. 4.8 also lack the heavy $\mathrm{TaC}$ particle decoration observed in $\mathrm{TaC}-3 \mathrm{G}$ or to a lesser degree in $\mathrm{TaC}-1 \mathrm{G}$.

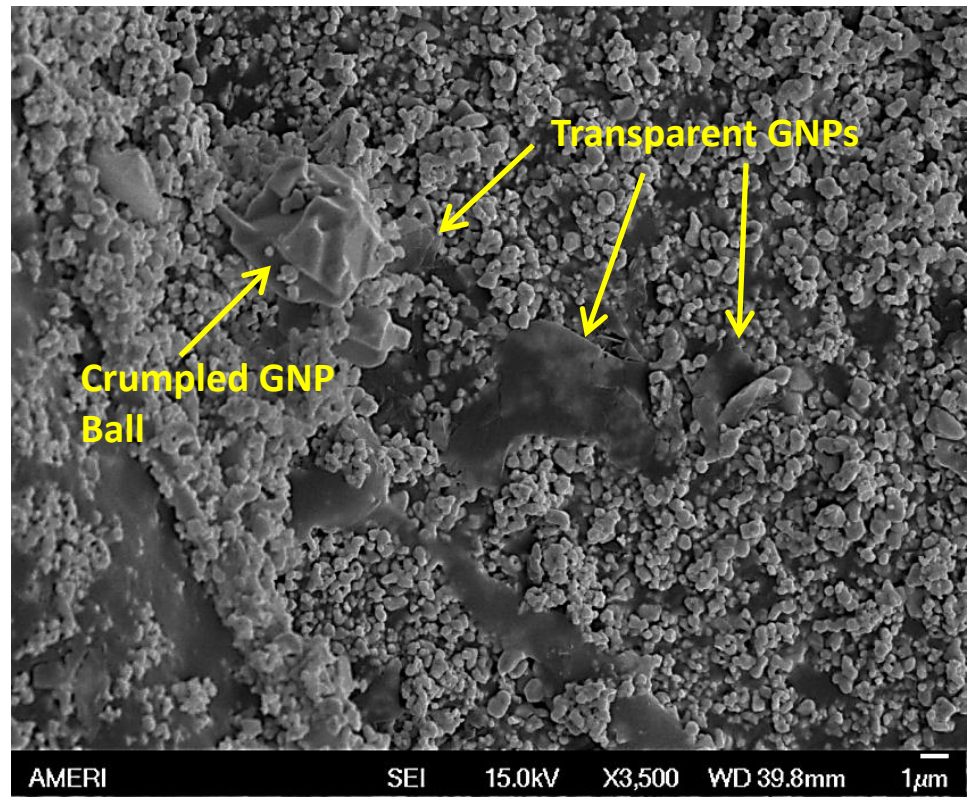




\subsubsection{High Energy Ball Milled Powder}

High energy ball milling was explored to see if GNP dispersion in the $\mathrm{TaC}$ powder could be improved without sacrificing the structural integrity of the GNP. The high energy ball milled powders display similar characteristics regardless of the amount of GNP content. The SEM micrograph in Fig. 4.9 shows that while the TaC-1G powder is homogenous, there are little signs of surviving GNPs. Fig. $4.9 \mathrm{~b}$ and $4.9 \mathrm{c}$ show that small flakes of GNP, on the order of $\sim 100 \mathrm{~nm}$, have survived and they are scattered throughout the surface of TaC particles. Similarly Fig. 4.10 shows traces of small GNP flakes in most of the TaC-5GNP powder. TaC-5G has a few areas where larger GNPs have survived as shown in Fig. 4.11, however similar to wet chemistry processing, the mixing between $\mathrm{TaC}$ and GNP is poor. The GNP particle in Fig. $4.11 \mathrm{~b}$ appears to be heavily agglomerated as the flat plate-like GNP structure has given way to a thick round particle.

The $\mathrm{TaC}$ particles in ball milled compositions appear to have experienced negligible levels of refining as the size and amount of submicron particles appears

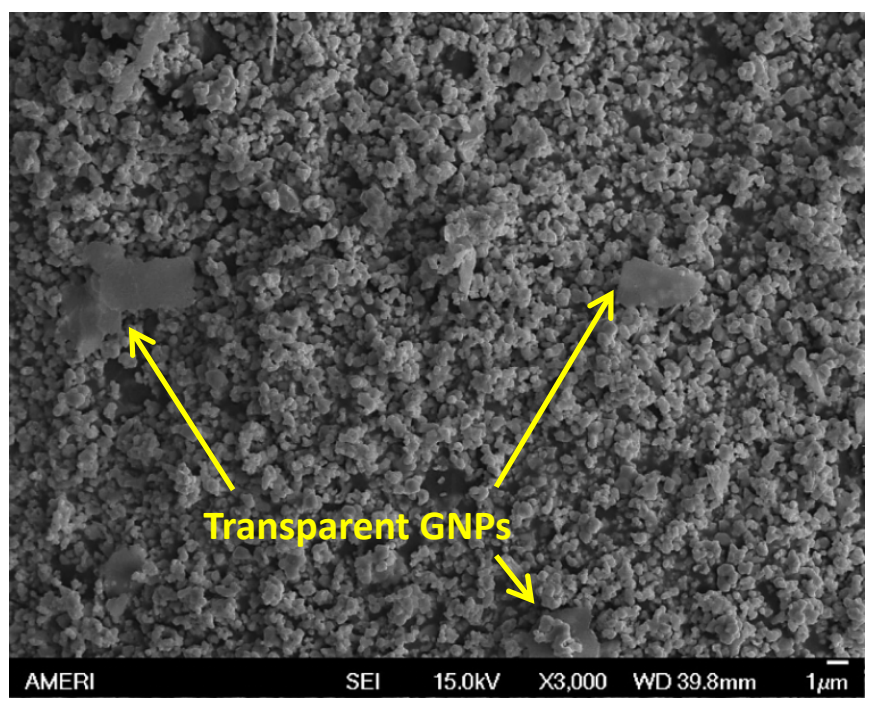




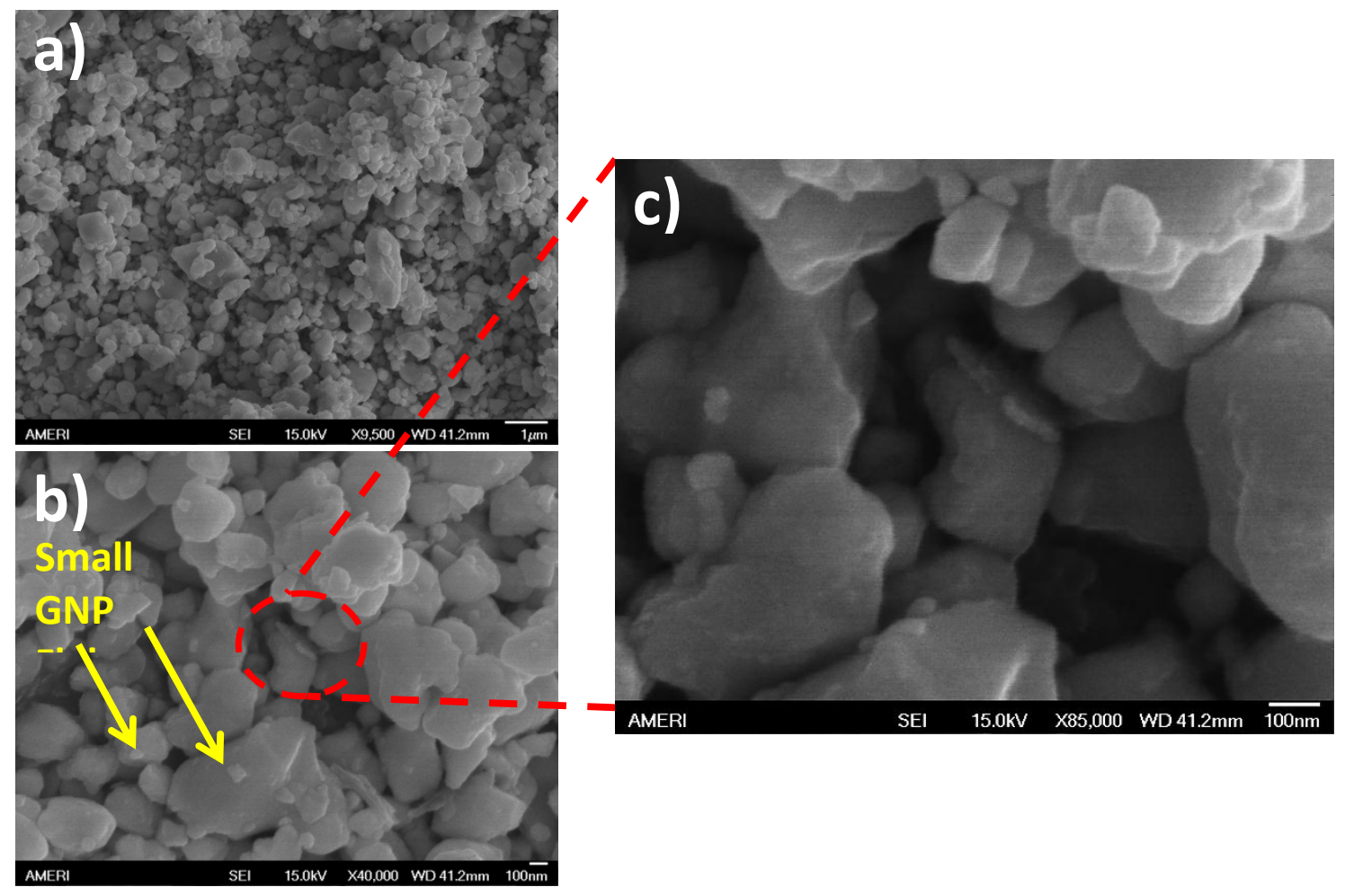

unchanged from the as received $\mathrm{TaC}$ powder. The $\mathrm{TaC}$ particles likely require longer milling times in order to achieve finer particles sizes which would improve the powder sinterability. Increasing milling times however, will only further reduce the size of the GNP particles which are a central pillar of the research. Hence, the ball milling route to GNP dispersion was not further pursued and the ball milled powders were not sintered.

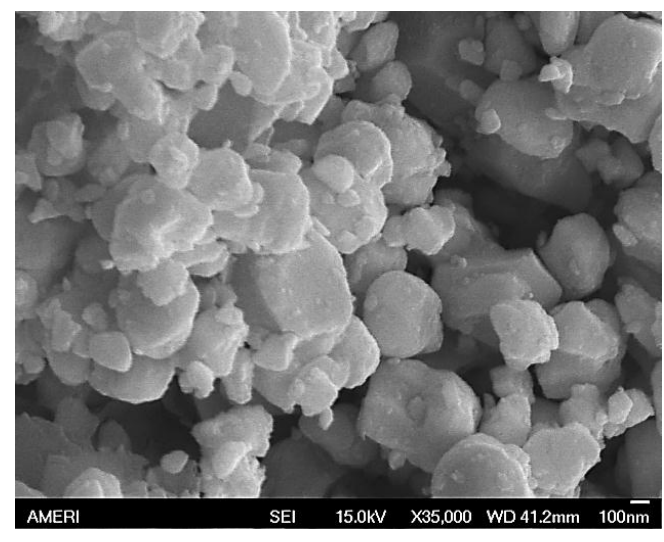



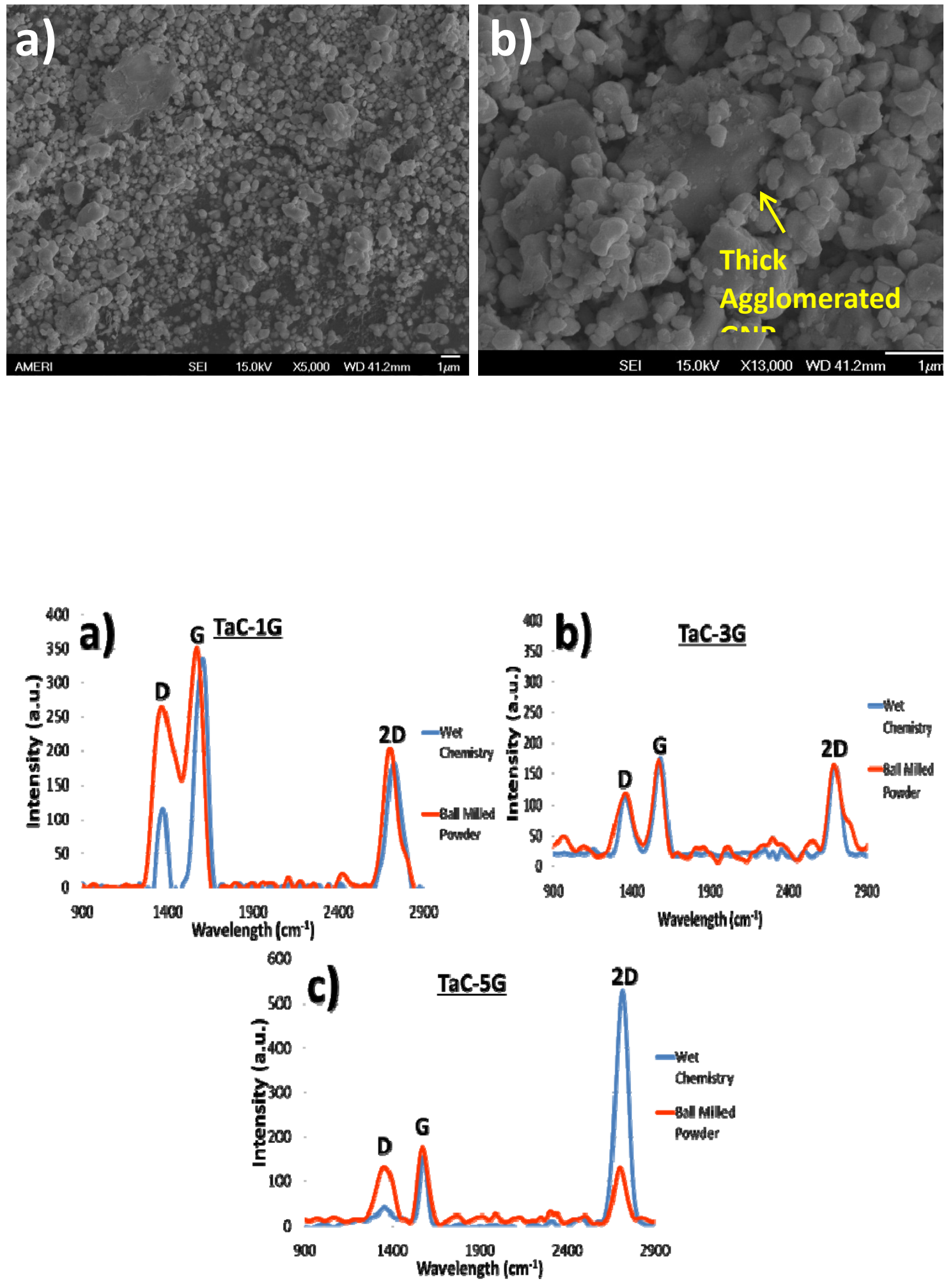


\subsection{Structure of GNP}

\subsubsection{Structure after ultrasonication}

Raman spectroscopy was employed to characterize the structure of GNP in the TaC-GNP powders. Raman spectra of TaC-1G, TaC-3G, and TaC-5G are provided in Fig. 4.12. A summary of the Raman peak values and the relevant peak intensity ratios is presented in Table 4.1. The ultrasonication of TaC-GNP powders leads to high interactions between GNP and TaC particles and these interactions appear to have varying effects on GNP. The Raman Spectra indicate that the presence of a graphene-like structure is presently mostly in the TaC-5G powder as indicated by the high $\mathrm{I}_{2 \mathrm{D}} / \mathrm{I}_{\mathrm{G}}$ ratio (3.4). A $\mathrm{I}_{2 \mathrm{D}} / \mathrm{I}_{\mathrm{G}}$ ratio below one indicates a graphitic structure and the $\mathrm{TaC}-3 \mathrm{G}$ and $\mathrm{TaC}-1 \mathrm{G}$ powders have $\mathrm{I}_{2 \mathrm{D}} / \mathrm{I}_{\mathrm{G}}$ ratios of .912 and .543 respectively. This increase in $\mathrm{I}_{2 \mathrm{D}} / \mathrm{I}_{\mathrm{G}}$ ratio with increasing GNP content indicates that the increased GNP content enables more GNP to elude major structural damage and thus retain their graphene-like structure. The presence of such large undamaged GNPs is widely seen in TaC-5G powder as shown in Fig. 4.5.

\begin{tabular}{|cccccc}
\hline Sample & D Peak & G Peak & 2D Peak & $\mathbf{I}_{\mathbf{D}} / \mathbf{I}_{\mathbf{G}}$ & $\mathbf{I}_{\mathbf{2 D}} / \mathbf{I}_{\mathbf{G}}$ \\
\hline $\begin{array}{c}\text { 1G } \\
\text { Powder }\end{array}$ & 1370.4 & 1604.3 & 2720.6 & 0.345 & 0.543 \\
$\begin{array}{c}\text { 3G } \\
\text { Powder }\end{array}$ & 1352.02 & 1581.9 & 2697.14 & 0.603 & 0.912 \\
$\begin{array}{c}\text { 5G } \\
\text { Powder }\end{array}$ & 1357.02 & 1579.56 & 2712.22 & 0.263 & 3.401 \\
\hline $\begin{array}{c}\text { 1G Ball } \\
\text { Milled }\end{array}$ & 1364.51 & 1569.01 & 2699.9 & 0.751 & 0.577 \\
$\begin{array}{c}\text { 3G Ball } \\
\text { Milled }\end{array}$ & 1357.02 & 1572.53 & 2691.6 & 0.656 & 0.955 \\
\hline $\begin{array}{c}5 G \text { Ball } \\
\text { Milled }\end{array}$ & 1353.27 & 1572.53 & 2698.52 & 0.725 & 0.715 \\
\hline
\end{tabular}


The Raman spectra indicate that ultrasonication causes some loss of graphene-like structure along with minor damage, however these are not the only effects. The presence of highly transparent GNP particles suggest that some GNP particles have experienced shearing forces during ultrasonication that result in reduced thickness and number of graphene layers in the GNP particles. Shearing of graphene layers leads to higher available surface area which may lead to stronger bonds between GNP and TaC. The transparent GNP are usually quite flat, indicating that they are thick enough to avoid becoming curled up as is known to occur with single layer graphene. GNP may also become highly wrinkled as shown in Fig. $4.4 \mathrm{~b}$ and this may have both adverse and favorable effects. The wrinkled structure indicates GNP have experienced heavy damage before even being sintering. The wrinkled particle in Fig. $4.4 \mathrm{~b}$ however appears to maintain favorable mixing with $\mathrm{TaC}$ particles and it is plausible that $\mathrm{TaC}$ particles would become entrapped within the fold and wrinkles leading to stronger interfaces and load transfer in the sintered compact.

\subsubsection{Structure after attrition milling}

Raman spectra of the ball milled TaC-GNP powders indicate that the GNP have experienced severe damage as a result of the attrition milling. The $I_{D} / I_{G}$ ratios are a measure of defects and damage in carbonaceous materials such as graphene and this ratio increases in all powders after ball milling. The GNP shown in Fig. 4.9 originated from the $\mathrm{TaC}-1 \mathrm{G}$ powder and it can be seen that the platelets are fractured into small pieces with significant wrinkling, while other have survived by becoming agglomerated low aspect ratio particles. 
The Raman spectra also indicate that the ball milling process does not result in additional loss of graphene-like structure as the $\mathrm{I}_{2 \mathrm{D}} / \mathrm{I}_{\mathrm{G}}$ ratios of $\mathrm{TaC}-1 \mathrm{G}$ and $\mathrm{TaC}-3 \mathrm{G}$ are similar before and after ball milling. Similar $\mathrm{I}_{2 \mathrm{D}} / \mathrm{I}_{\mathrm{G}}$ ratios suggests that ball milling processing damages GNP mostly by crushing and fracturing rather than sliding and shearing of graphene sheets. The lack of GNP shearing can be seen from the $\sim 150 \mathrm{~nm}$ wide GNP in Fig. 4.9c, as despite the reduction in width the platelet is still 20-30 nm thick. This thickness may indicate that this particular GNP particle may have actually consisted of several platelets before becoming fractured into a small thick platelet. The TaC-5G powder had many large undamaged GNP after the wet chemistry processing which resulted in the large $\mathrm{I}_{2 \mathrm{D}} / \mathrm{I}_{\mathrm{G}}$ ratio; these large particles likely experienced varying degrees of crushing, shearing, and agglomeration during the ball milling processing resulting in the drastic loss of graphene-like structure. The thick round GNP seen in Fig. $4.11 \mathrm{~b}$ corroborates that GNP became agglomerated in the TaC-5G samples

Overall, SEM and Raman spectroscopy indicate that GNP particles have experienced damage during ball milling that results in greatly diminished GNP size. The GNP consists of mostly small flakes and the interaction and mixing with the $\mathrm{TaC}$ particles appears to be minimal. One of the prime points of interest of this study is the effect of graphene nanoplatelets on the toughening of TaC-GNP composites and thus the survival of GNP is vital. The high energy ball milling process further damages GNP and provides no tangible advantage over the wet chemistry approach and therefore the study proceeded without the ball milled powders and hence only the wet chemistry powders were consolidated into bulk samples. 


\section{Synthesis of Bulk GNP}

\subsection{Bulk Structure Consolidation}

A bulk GNP structure was consolidated in order to examine the feasibility and structure retention of GNP processed under the extreme conditions needed to consolidate a UHTC such as TaC. Fig. 4.13 shows the variation of temperature, applied pressure, and the punch displacement during SPS processing. The temperature was gradually increased up to $1100{ }^{\circ} \mathrm{C}$ before increasing the pressure. The pressure is then ramped up to $80 \mathrm{MPa}$, the temperature is held steady during the increase in pressure. It can be seen from the punch displacement, that the powder in the die is compressed significantly (over $2 \mathrm{~mm}$ ), when the pressure was ramped up. Once the desired pressure was reached, the temperature was further increased to the maximum required temperature of $1850{ }^{\circ} \mathrm{C}$.

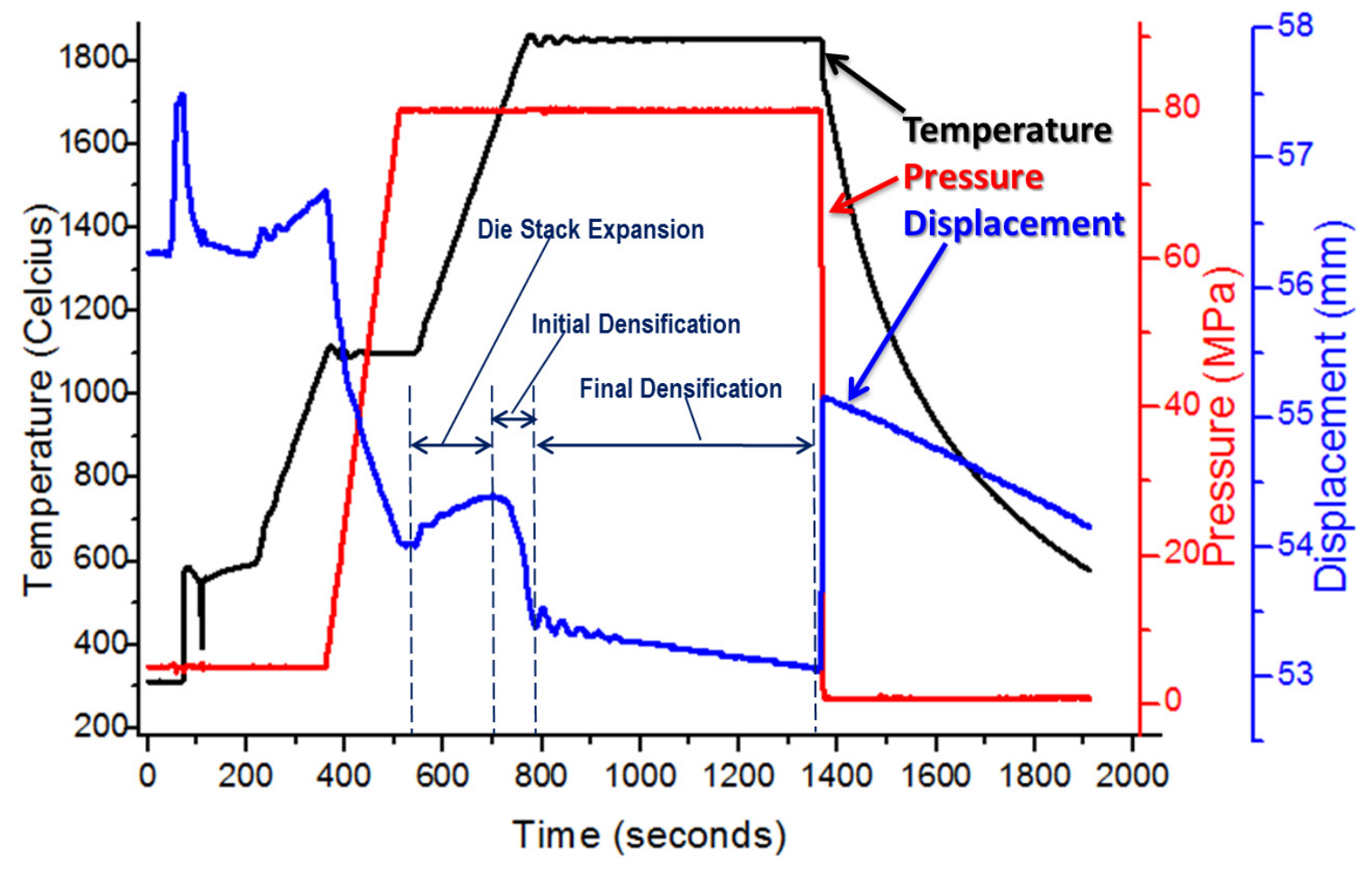




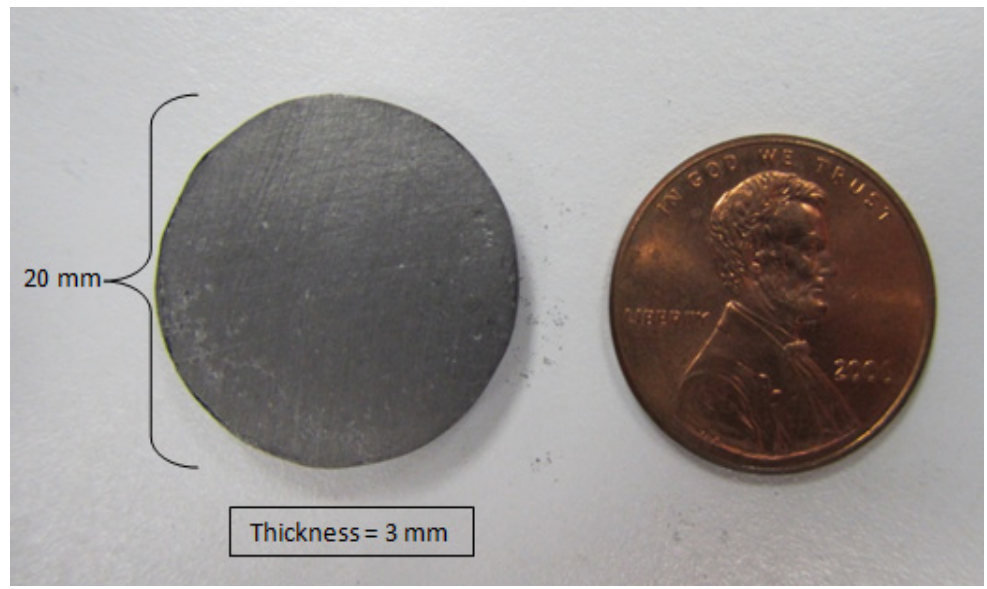

During this increase in temperature, there is a positive change in the punch displacement, due to expansion of the graphite die stack assembly. However, at the tail end of the heating period $\left(1650^{\circ} \mathrm{C}\right.$ to $\left.1850^{\circ} \mathrm{C}\right)$, there is a substantial negative change in the punch displacement, indicating that the majority of the powder compression occurs at this time. The powder is then sintered at the desired holding pressure and temperature for approximately 10 minutes. During this sintering period, the powder steadily continues to compress until the sintering process is complete; at which point the pressure is released and the temperature gradually ramped down. The resulting bulk GNP pellet is shown in Fig. 4.14, along with the actual dimensions. The density of the starting GNP powder was measured using a Helium Pycnometer, and found to be $1.82 \mathrm{~g} / \mathrm{cm}^{3}$. The true density of the bulk GNP pellet was found to be $2.11 \mathrm{~g} / \mathrm{cm}^{3}$. The increase in the density is largely attributed to the consolidation of powder. The formation of high density phases of carbon was not observed as discussed in the next section. 


\subsection{Bulk GNP Microstructure}

Figures $4.15 \mathrm{a}$ and $4.15 \mathrm{~b}$ show low magnification SEM images of the fracture surface of SPS consolidated GNPs. The pellet shows dense structure at the top and bottom surfaces with stacks of GNPs in the intermediate. GNPs are uniformly oriented as layered structure throughout the thickness of the pellet. Fig. 4.16a shows the top surface of the consolidated GNP structure. GNPs retain their platelet shape on the top surface with some signs of damage at the edges. A high magnification SEM image of the crosssection of the layered GNP stack shows undamaged and oriented GNPs in Fig. 4.16b. It can be concluded from Fig. 4.16 that GNP experience very little damage during the SPS processing.

The structure of the GNPs before and after SPS consolidation is also examined by micro Raman Spectroscopy. Raman spectra from both GNP powder and SPS-pellet show
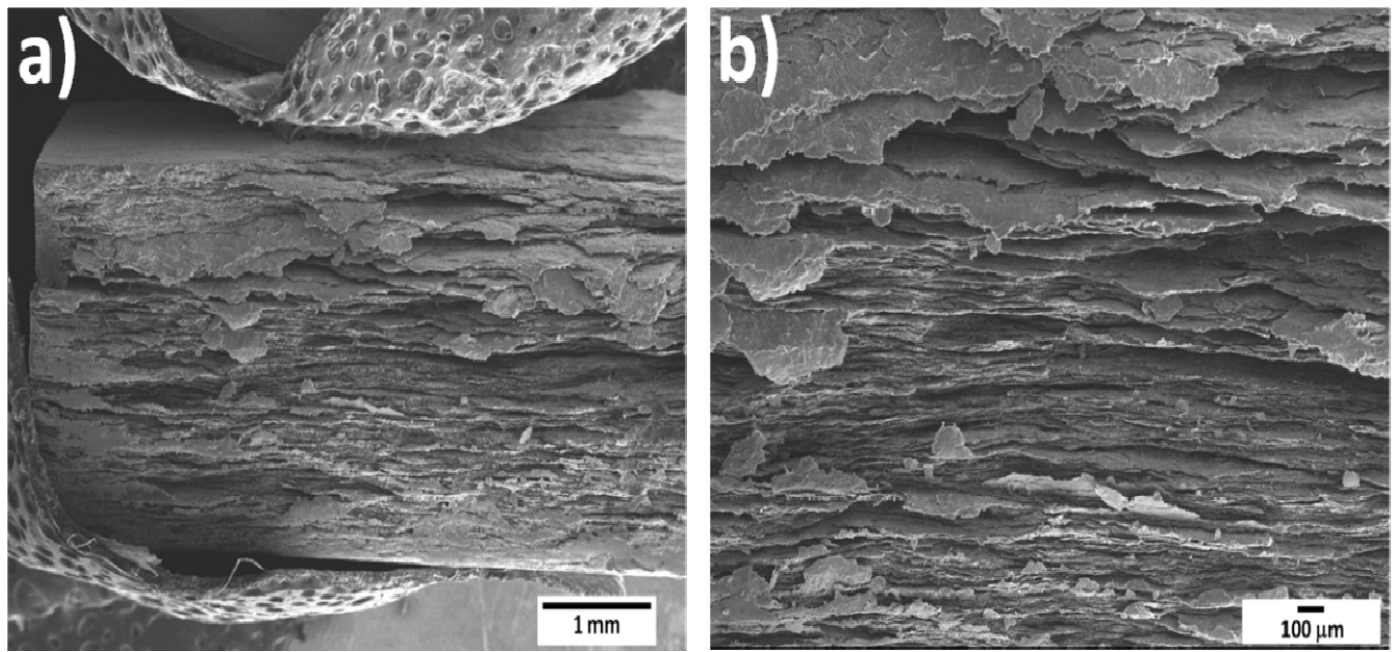

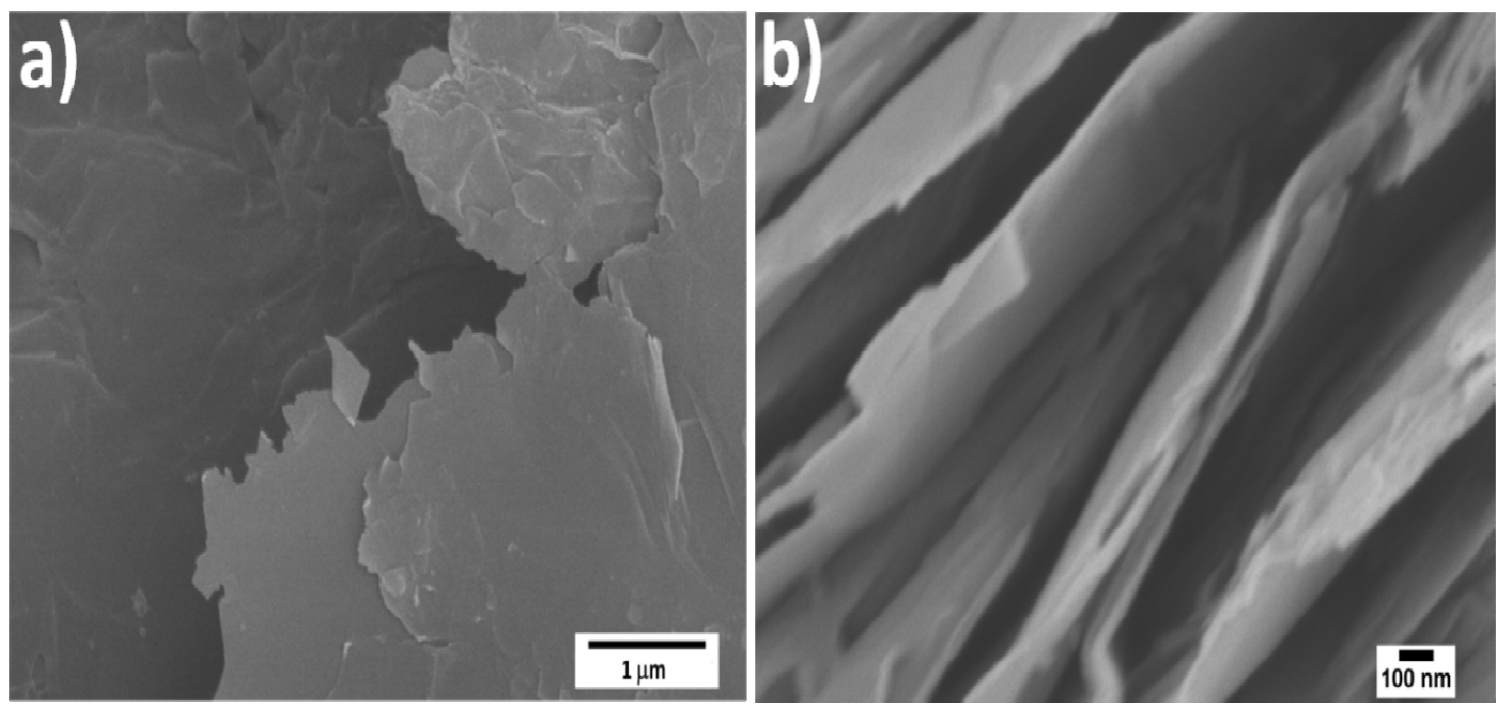

presence of D, G and 2D peaks (2D peak also known as G') (Fig. (4.17)) at the positions which are the signatures of graphite like structures [85-87]. The G and 2D peaks appear from the in-plane $\mathrm{C}-\mathrm{C}$ bond stretching in graphene, whereas the D peak corresponds to the defects introduced in the structure. A very small intensity D peak as compared to $G$ peak in GNP-powder indicates very small amount of defects present at the powder stage. Slightly higher D peak in the case of pellet might indicate some amount of defect introduced during SPS process. But the thermal annealing during cooling cycle of SPS might help in releasing pressure induced stress on GNP structure. In fact, the $\mathrm{I}_{\mathrm{D}} / \mathrm{I}_{\mathrm{G}}$ ratio for both powder and sintered structure remains similar $(0.125)$ indicating no significant change to the GNP structure during SPS. This observation along with similar intensity of G peaks (the crystallization peak for graphene) for powder and pellet indicates retention of graphene in the SPS consolidated structure. 


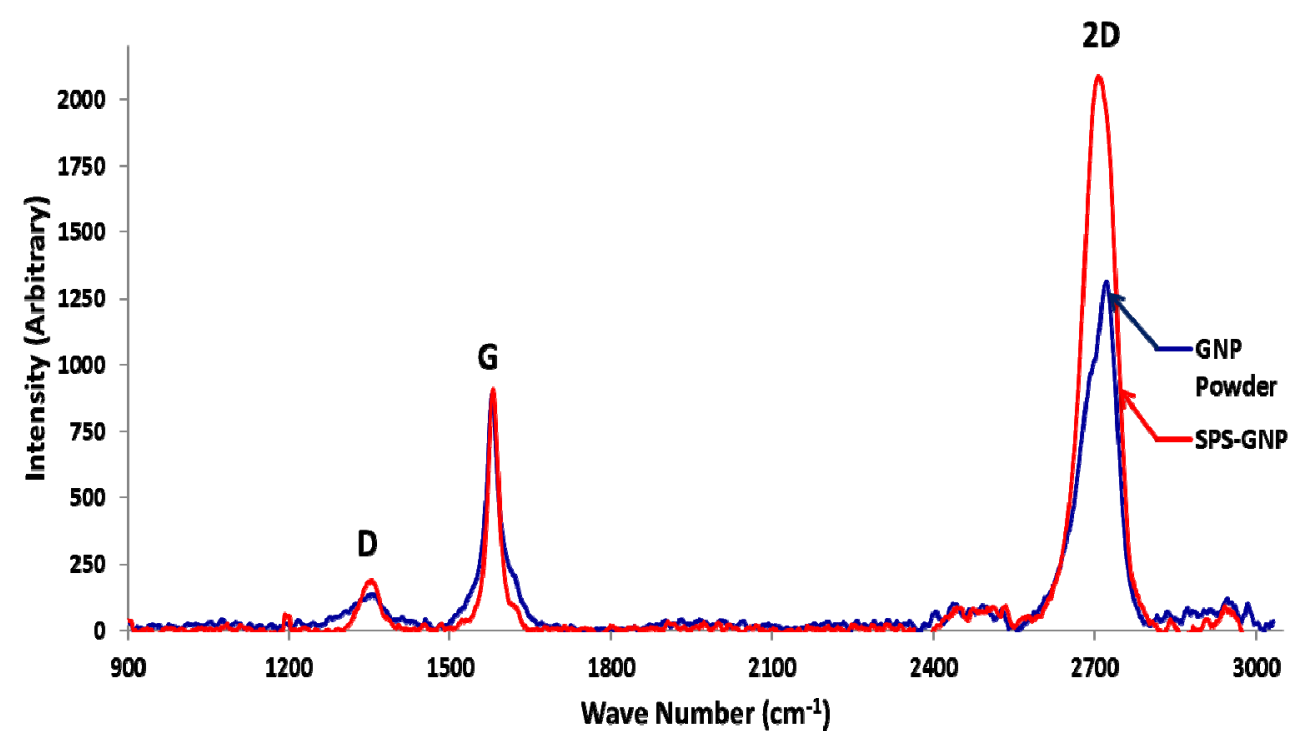

Retention of graphene structure is further confirmed by the presence and intensity of 2D peak. Both samples show presence of 2D peak at $\sim 2700 \mathrm{~cm} \neg-1$, as reported for graphene Raman spectra at $514 \mathrm{~nm}$ [24]. The intensity ratio of 2D/G peak is almost 4 for single layer graphene [24] and decreases with an increase in the number of layers [2427]. The intensity of $2 \mathrm{D}$ band becomes lower than $\mathrm{G}$ for bulk graphite [24-27]. Moreover, the 2D peak in single layer graphene is a narrow and sharp one, which gets broadened and shows sign of splitting with increasing number of graphene layers [24-27]. In the present study, both the powder and pellet shows broadened 2D peak with sign of splitting, indicating presence of multiple graphene layers. But, the intensity ratio of $2 \mathrm{D} / \mathrm{G}$ is still greater than 1 , indicating the retention of graphene structure and not fully transforming to bulk graphite. Hence, it can be concluded from Raman Spectroscopy that GNP structure is largely retained after SPS consolidation. 


\section{Consolidation of TaC-GNP Composites}

The bulk TaC-GNP samples consolidated by Spark Plasma Sintering (SPS) are shown in Fig. 4.18. The graphite foil used to prevent sintered powder from contacting the graphite die leads a thin layer of graphite appearing on the surface of the sintered compacts. Even after lightly grinding the bulk pellets, there is some graphite remaining due to the uneven surface contours of the sample. Complete removal of the graphite deposits would require the sample surface to be grinded until flat which would result in unnecessary loss of material as well as potential damage to the brittle samples.

Table 4.2 shows the fine density of each sample along with the corresponding degree of densification. The sample thickness is also included, each sample had a diameter of $\sim 20 \mathrm{~mm}$. The density of the TaC-1G, TaC-2G, and TaC-3G samples reflect densification of $\sim 97 \%$; this is considered a high level of densification for UHTCs and is on par with results from methods employing longer times and higher temperatures (i.e.
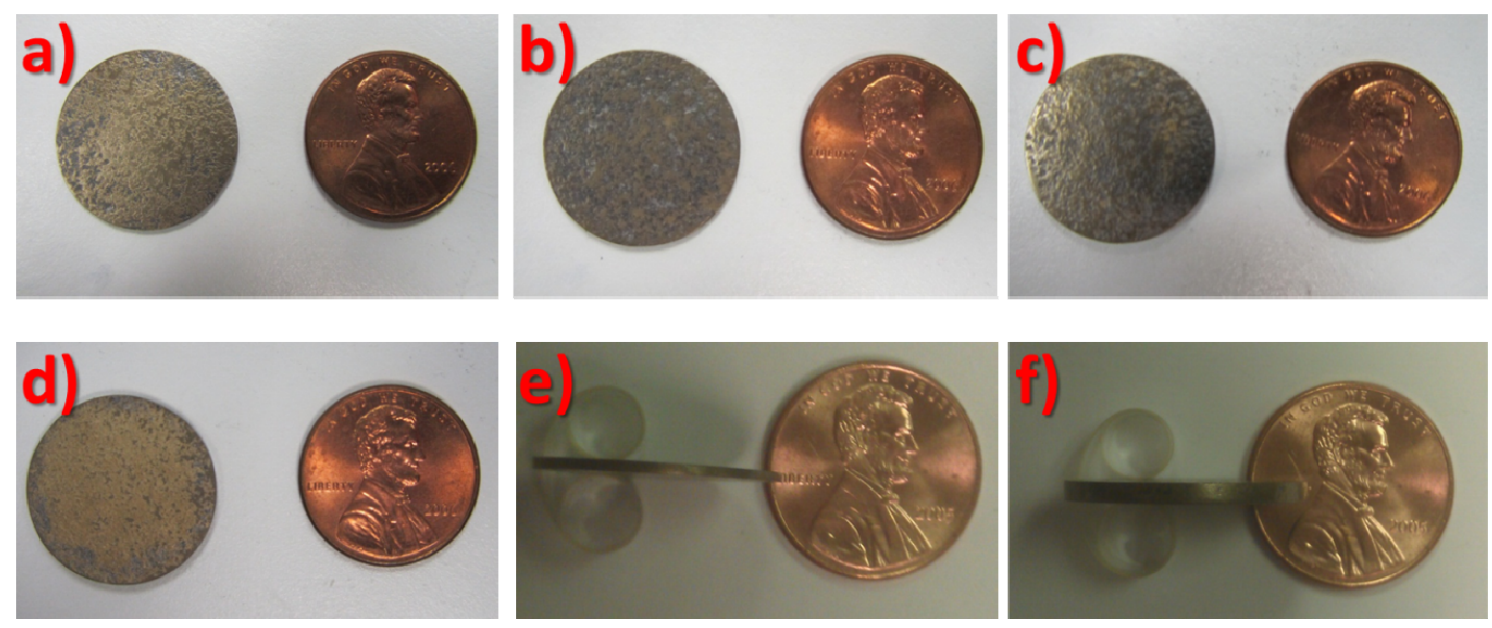


\begin{tabular}{|c|c|c|c|}
\hline Sample & Thickness (mm) & Density (g/cc) & $\begin{array}{c}\text { Percent } \\
\text { Densification }\end{array}$ \\
\hline $\mathrm{TaC}-\mathbf{1 G}$ & 1.12 & $14.085+/-.064$ & $96.86+/-.44$ \\
\hline $\mathrm{TaC}-\mathbf{2 G}$ & 1.56 & $14.085+/-.033$ & $97.72+/-.23$ \\
\hline $\mathrm{TaC}-3 \mathrm{G}$ & 1.62 & $13.921+/-.059$ & $97.45+/-.41$ \\
\hline $\mathrm{TaC}-4 \mathrm{G}$ & 1.75 & $13.150+/-.065$ & $92.90+/-.44$ \\
\hline $\mathrm{TaC}-5 \mathrm{G}$ & 1.57 & $13.860+/-.035$ & $98.80+/-.24$ \\
\hline $\mathrm{TaC}$ & 0.82 & $13.842+/-.099$ & $94.35+/-.68$ \\
\hline TaC Powder & $N / A$ & 14.67 & $N / A$ \\
\hline GNP Powder & $N / A$ & 1.82 & $N / A$ \\
\hline
\end{tabular}

hot pressing) as discussed in Chapter 2. These three samples were sintered at the desired temperature of $1850{ }^{\circ} \mathrm{C}$ and pressure of $100 \mathrm{MPa}$ as shown in Fig. 3.6 in Chapter 3. The processing charts in Chapter 3 reveal that the majority of the densification has occurred once the pressure of $100 \mathrm{MPa}$ is reached and the temperature during this period is ramping up from $1100^{\circ} \mathrm{C}$ to $1850{ }^{\circ} \mathrm{C}$. The samples continue to steadily densify during the 10 minute hold as indicated by the increased displacement of the graphite punches. The high level of densification is confirmed by the optical micrographs shown in Fig. 4.19a-f. The images reveal high densification has occurred as little to no porosities are observed in these images; the spherical dark regions seen are a result of ceramic chipping during grinding and polishing of the samples.

The TaC-5G sample (98.8\%) had the highest densification of all the samples and this is surprising considering that this sample was sintered at a pressure of $80 \mathrm{MPa}$ and not $100 \mathrm{MPa}$ as discussed in Chapter 3. It is possible that the higher GNP content significantly increases the conductivity of the sample and the distribution of current throughout the sample is more uniform relative to the samples with lower GNP concentrations. As discussed in Chapter 2, samples with higher electrical conductivity 

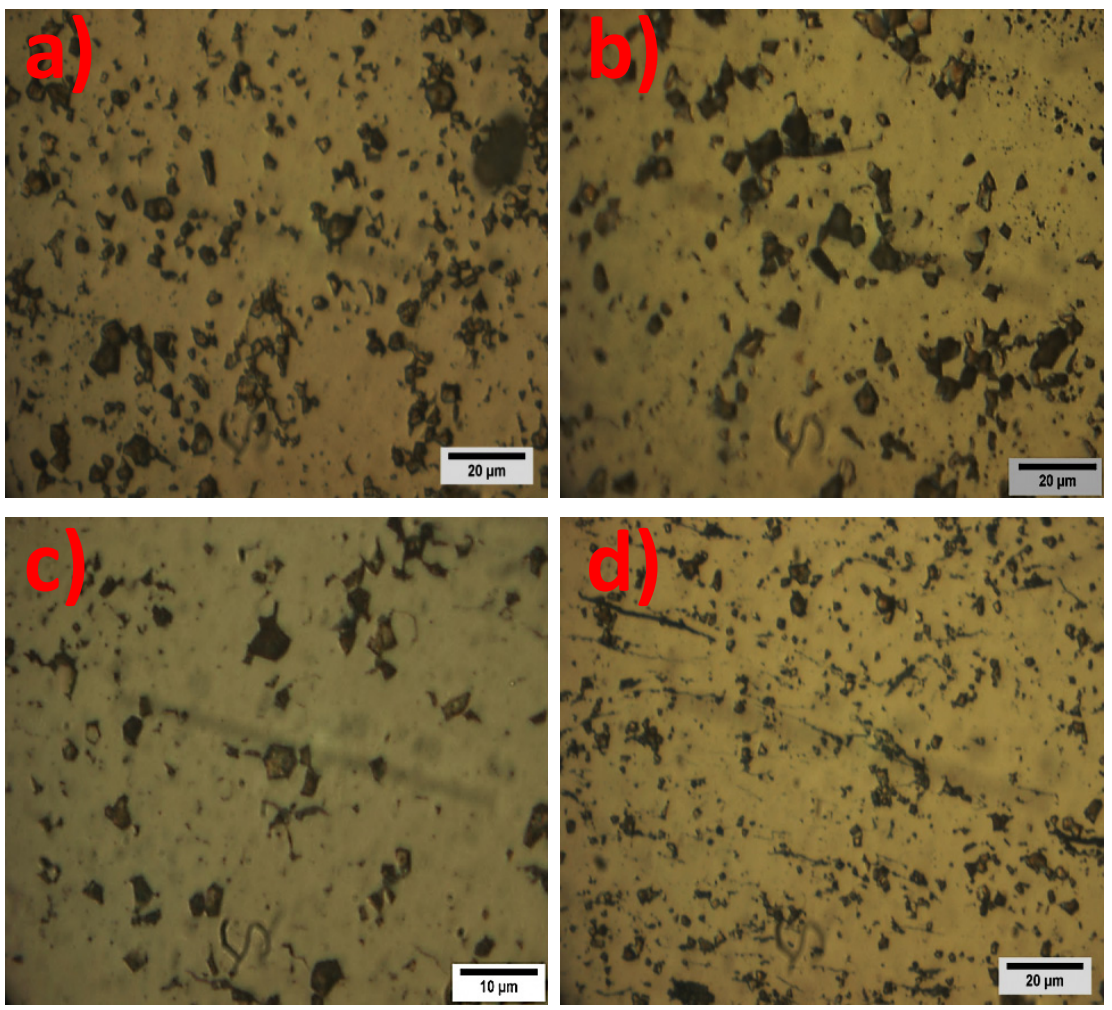

experience higher and more uniform heating during SPS processing and this results in higher densification. The optical micrographs of the cross section shown in Fig. 4.19d reveal a highly densified structure. Aside from the absence of porosity, and the chipped features, the TaC-5G sample displays several "streaking" and aligned features which are thick, long GNPs. These features are hardly seen in any of the other samples and this is consistent with the observations of the starting powders as thick GNPs were seen only in the TaC-5G powder.

The densification of the TaC sample allows the effect of GNP on sintering and consolidation to be gaged. The $\mathrm{TaC}$ sample was processed under the same conditions as the TaC-5G sample yet as seen in Fig. 4.20 the densification is significantly lower (94. 35\%). The processing charts clearly indicate that the TaC-5G sample undergoes a 
substantially higher initial densification, while the final densification stages are similar. The samples were sintered using the same SPS machine so variations due to SPS machine used are unlikely. The addition of GNP is the only difference between the two samples which gives credence to the enhancing effect of GNP on sintering and consolidation.

The $\mathrm{TaC}$ sample in this study achieves a higher densification then a $\mathrm{TaC}$ sample processed under similar conditions by Bakshi et al [69]. In that study the $\mathrm{TaC}$ was processed at a temperature of $1850{ }^{\circ} \mathrm{C}$ and a pressure of $100 \mathrm{MPa}$, yet the densification achieved was only $89 \%$. The consistency of SPS processing is known to vary, however this level of variation is unlikely to be predominantly due to SPS or machine variation. The primary difference may be in the processing of the powder, as in the present study the $\mathrm{TaC}$ powder was ultrasonicated for 90 minutes. Bakshi et al [69] do not ultrasonicate

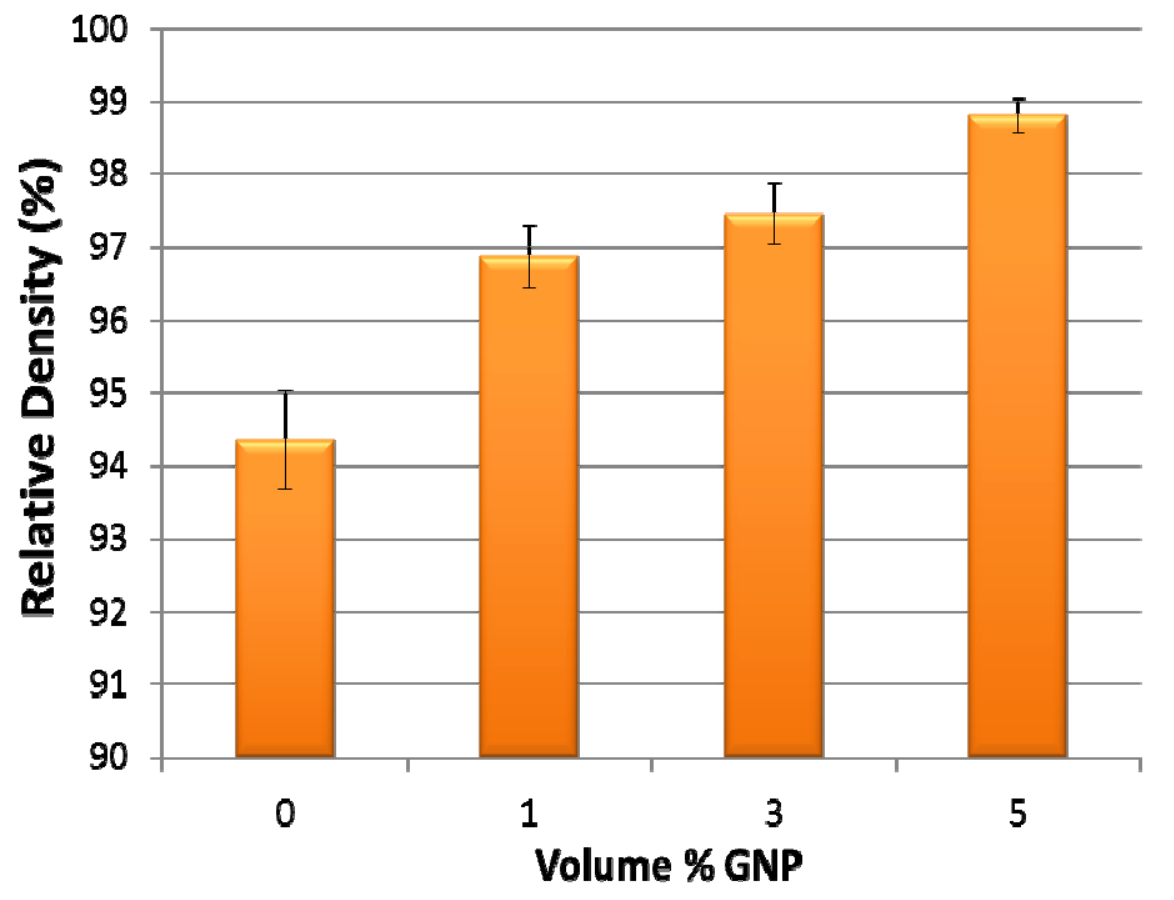


the $\mathrm{TaC}$ powder and instead sinter the powder as received. The ultrasonication dissembles the $\mathrm{TaC}$ agglomerates present in the as received powder, hence increasing available surface area and enhancing the powder's sinterability.

The TaC-4G sample experiences the lowest level of densification (92. 9\%) and this is due to the abrupt end to the SPS processing which was discussed in Chapter 3. The sample still achieves relatively high densification and this is due to the fact the most of the densification occurs in the initial stage prior to the 10 minute hold. The first stage of processing is the same as the $\mathrm{TaC}-1 \mathrm{G}, \mathrm{TaC}-2 \mathrm{G}$, and $\mathrm{TaC}-3 \mathrm{G}$ samples and thus the effect of the initial densification stage can be quantified. The $\sim 92 \%$ densification achieved by the TaC-4G sample is representative of the densification achieved in the initial stage, and therefore the remaining final densification accounts for an additional 4-5\% densification. This sample's processing and densification is substantially different from the rest of the samples and it was omitted from any further experiments or analysis. In addition, the TaC-2G sample was also omitted from further analysis because of its similarity to TaC$1 \mathrm{G}$ and $\mathrm{TaC}-3 \mathrm{G}$ in the early stages of analysis.

\section{TaC-GNP Composites}

\subsection{Microstructure}

The focus of this section is on the effect of varying concentrations of GNP on the microstructure of the TaC-GNP composites. The microstructures of the formed TaC-GNP composites are characterized by observing the fracture surfaces in a scanning electron 
microscope (SEM). The microstructure of the pure TaC sample is discussed first in order to provide a baseline of the type of microstructure obtained from SPS processing using the parameters previously discussed $\left(80 \mathrm{MPa}, 1850{ }^{\circ} \mathrm{C}\right)$. The slight difference in applied pressure during the SPS processing for $\mathrm{TaC} / \mathrm{TaC}-5 \mathrm{G}(80 \mathrm{MPa})$ and $\mathrm{TaC}-1 \mathrm{G} / \mathrm{TaC}-3 \mathrm{G}(100$ $\mathrm{MPa}$ ) is not expected to have a substantial effect on the overall microstructures of the sintered compacts.

Fig. 4.21 shows the typical microstructure of the sintered $\mathrm{TaC}-$ consolidated structure with highly defined grains is observed. It can be seen that most of the structure was sintered into a consolidated structure with highly defined grains. There are however,

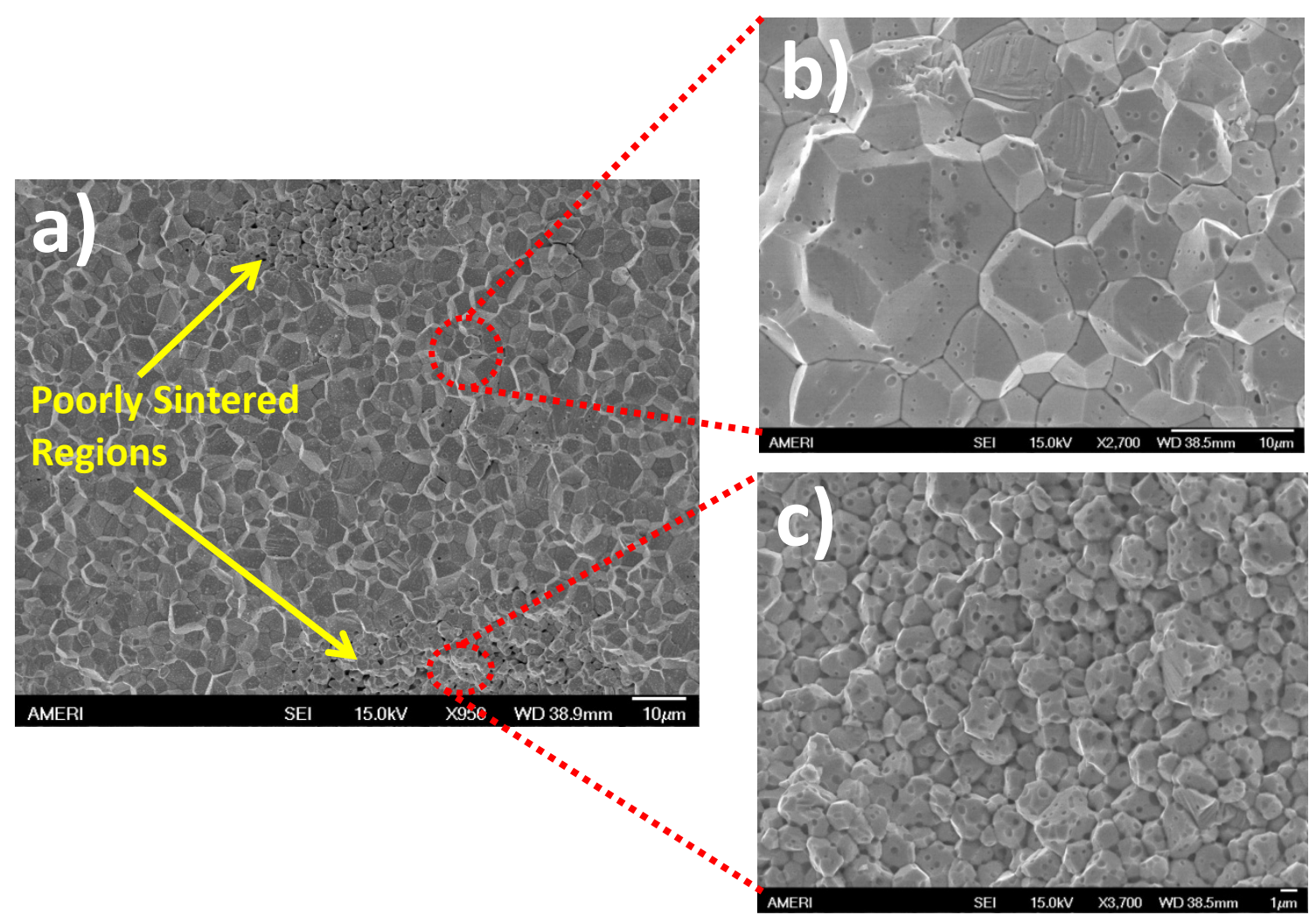




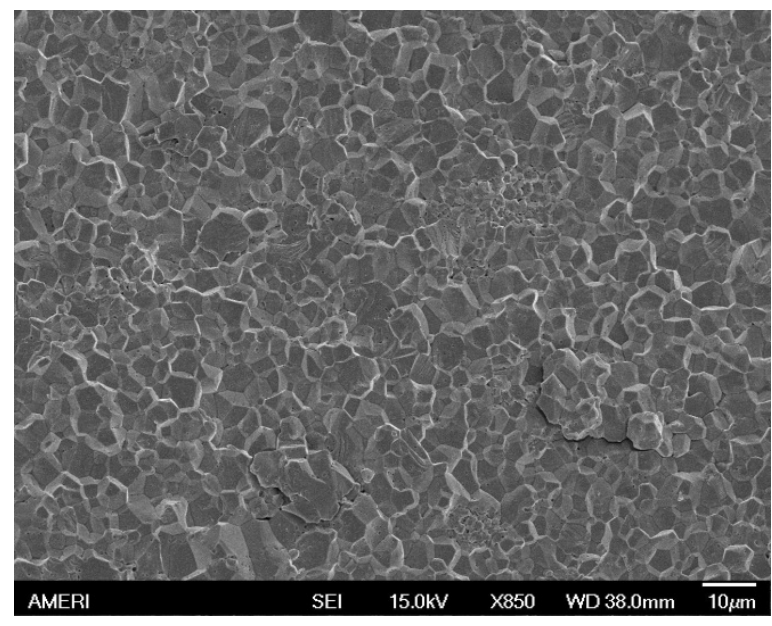

some regions where sintering did not occur effectively resulting in a porous granulated structure. Fig. 4.21b shows a high magnification micrograph of a dense TaC region; a clean intergranular fracture with no porosities is observed. Fig. 4.21c shows a poorly consolidated region; the bonding between granules is poor and the grain size is substantially lower than in the highly consolidated regions. This variation leads to a bimodal distribution of grain sizes; with the dense regions having grain size of $4.54 \pm$ $0.91 \mu \mathrm{m}$ and the poorly sintered regions having grain sizes of $1.43 \pm 0.38 \mu \mathrm{m}$. The structure observed here displays a much higher level of consolidation ( $94 \%)$ than the TaC structure processed under the same conditions by Bakshi et al [69] which has a yield of $\sim 89 \%$ densification.

The microstructure of TaC-1G, shown in Fig. 4.22, corresponds with the high densification (96.86\%) achieved by this sample. Table 4.3 and Fig. 4.23 shows that this sample had similar grain size as the $\mathrm{TaC}$. The grain size here however is more uniform as there were minimal areas with poor consolidation. The TaC-1G grain size is lower than 


\begin{tabular}{lcc}
\hline Sample & Average $(\mu \mathrm{m})$ & Densification(\%) \\
\cline { 2 - 3 } TaC & $3.33+/-1.65$ & 94.35 \\
$\begin{array}{l}\text { Poorly sintered } \\
\text { Regions }\end{array}$ & $1.43+/-0.38$ & - \\
Well Sintered Regions & $4.54+/-0.91$ & - \\
TaC-1G & $3.38+/-1.57$ & 96.86 \\
TaC-3G & $1.44+/-0.62$ & 97.45 \\
TaC-5G & $1.41+/-0.67$ & 98.80 \\
\hline
\end{tabular}

that of the well sintered grains in TaC.The presence of GNP is likely the reason for decreased grain size in spite of the higher densification - this will be further elucidated in terms of microstructure and mechanisms upon observing the $\mathrm{TaC}-3 \mathrm{G}$ and $\mathrm{TaC}-5 \mathrm{G}$ microstructures.

The presence of GNP is not immediately seen in the low magnification image in Fig. 4.22; high magnification images in Fig. 4.24 however reveal there are significant amounts of GNP distributed throughout the structure. The largest GNP structure in Fig. $4.24 \mathrm{c}$ is approximately $400 \mathrm{~nm}$ wide with a thickness of about $50 \mathrm{~nm}$, likely indicating

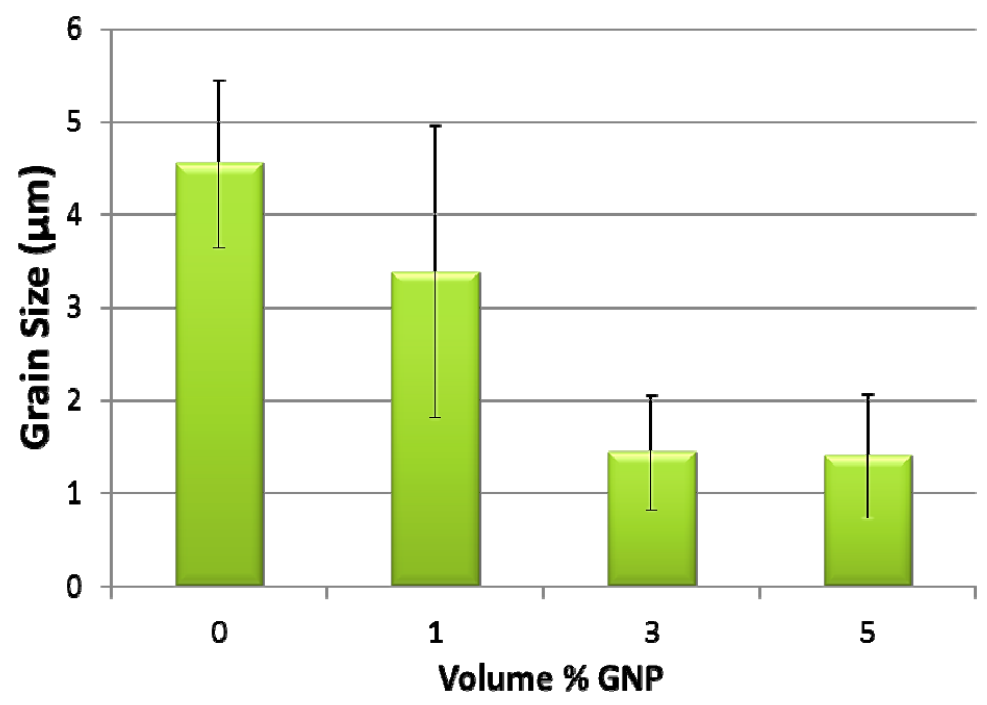




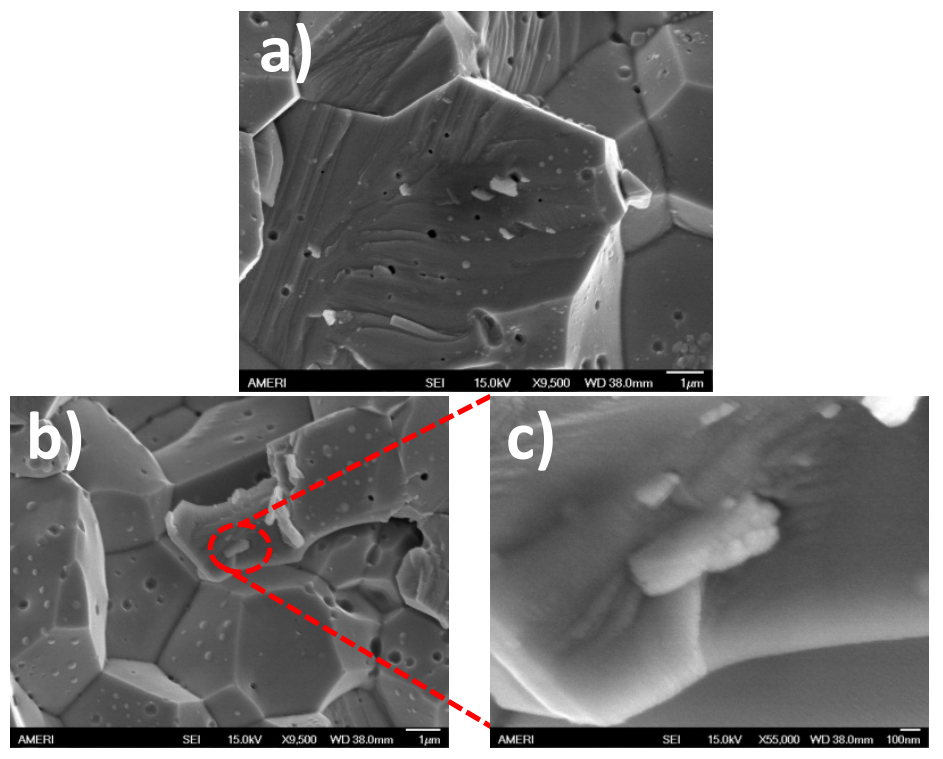

that a few platelets have stacked together. Smaller GNP, possibly single platelets can be seen surrounding the larger GNP in Fig. 4.24c. The GNP inte ract with the TaC grains in two distinct manners; some become embedded in the grains as in Fig. 4.24 and Fig. 4.25a, whiles others lie in between TaC grains as in Fig. 4.25b. GNP embedded in grains may pin two or more grains together and strengthen the structure. Embedded GNPs tend to be found in transgranular or fractured grains as in Fig. 4.25a. The GNP may enhance $\mathrm{TaC}$ grain adhesion and cause the fracture mode to switch to transgranular; a strong GNP/TaC interface will effectively transfer the load to the GNP 'in-plane' direction where the elastic modulus is highest.

The GNP in between grains may wrap around $\mathrm{TaC}$ grains and provide similar enhancements to TaC grain adhesion. The GNP in between grains however will not have as strong a GNP/TaC interface as the embedded GNPs, and thus the load transfer to the GNP will be reduced in this configuration. It is also possible that if insufficient GNP 

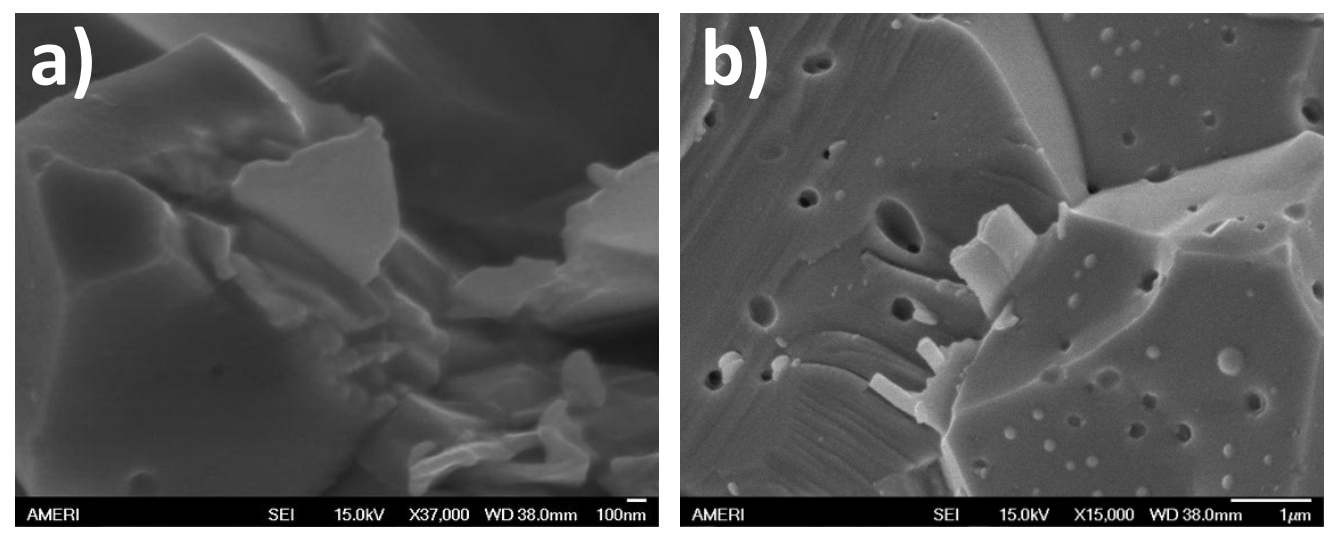

wrapping has occurred, the shearing off of platelets or graphene sheets may induce grain slipping hence weakening the structure.

The impact of GNP on the microstructure is most clearly seen in the TaC-3G sample. The micrographs in Fig. 4.26 reveal that the GNPs are not only well distributed throughout the $\mathrm{TaC}$ matrix, but they are also connected over long length scales. The GNPs in TaC-3G appear to form a coherent GNP network throughout the structure unlike the isolated platelets seen in TaC-1G. The high magnification micrograph in Fig. 4.26b shows several 4-6 $\mu \mathrm{m}$ long GNPs weaving in between several TaC grains. The uniformity of the GNPs throughout the samples is a product of the uniform GNP dispersion observed in the starting TaC-3G powder. The micrograph in Fig. 4.26d reveals how two separate GNP have come into contact and formed a coherent interface. Both these GNP are embedded in grains and thus the pinning effect is enhanced as each GNP pins two grains with the grain in the middle being shared by both GNP. Small isolated embedded GNPs, like those seen in TaC-1G, can also be seen in a $\mathrm{TaC}$ grain in Fig. $4.26 \mathrm{~d}$. 

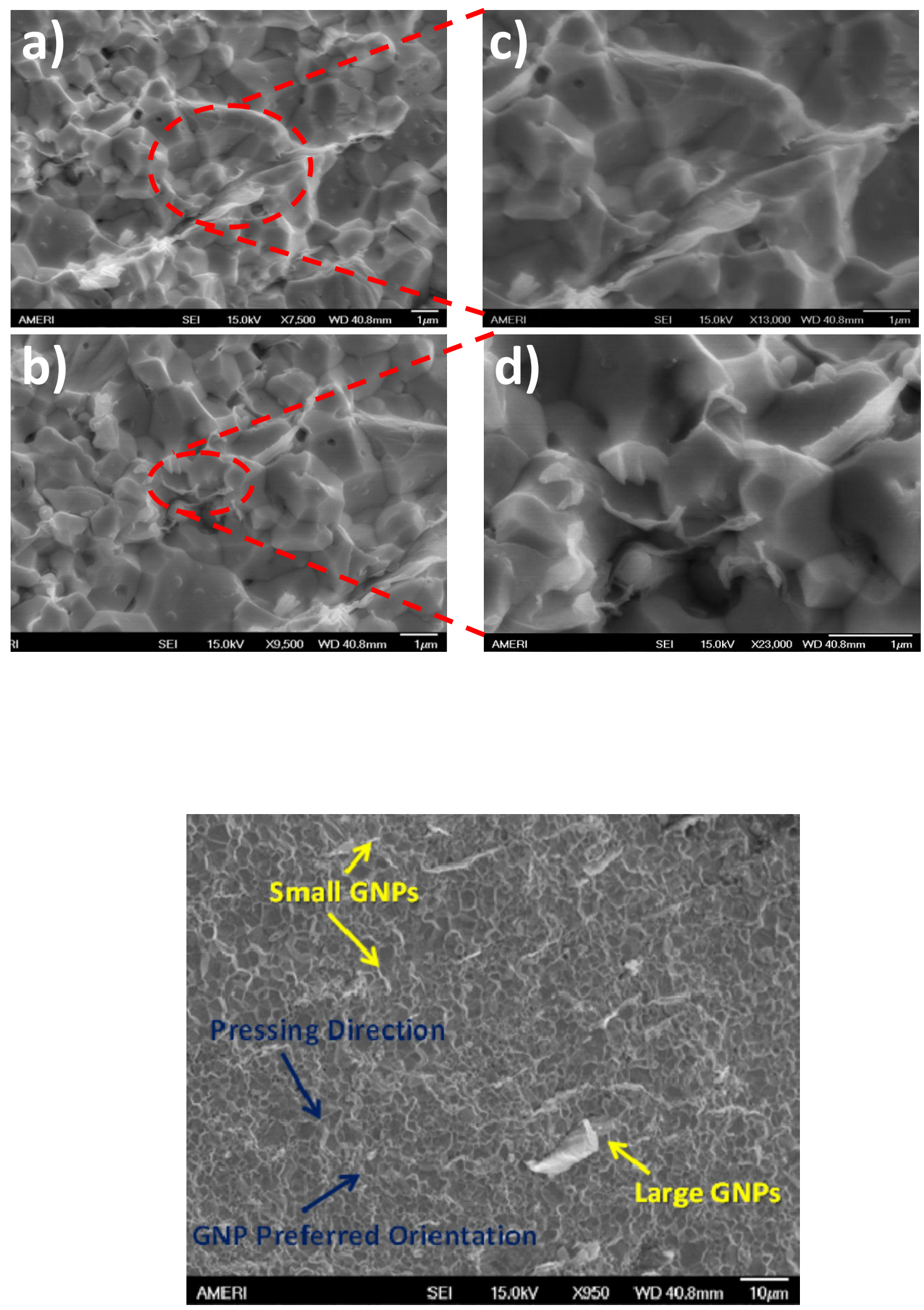
The weaving GNPs in the TaC-3G structure reveal the GNP wrapping mechanism widely reported in the literature $[106,107,109]$. The GNP wrapping is likely the reason why the $\mathrm{TaC}$ grain size $(1.44+/-0.62 \mu \mathrm{m}$ for $\mathrm{TaC}-3 \mathrm{G})$ continues to decrease with increasing GNP content. When GNPs wrap around grains, they act as diffusion barriers and hinder further grain growth of the materials. The ability to hinder grain growth while ensuring high densification is ideal and significant; high grain growth is normally a byproduct of effective sintering as the driving mechanisms of sintering and grain growth are coupled. The high surface area and high thermal conductivity of GNP allow for strong interfacial bonding between $\mathrm{TaC}$ grains and GNP which minimizes porosity formation.

Other interesting features of the TaC-3G microstructure can be seen in Fig. 4.27, including the apparent orientation of GNPs during sintering. The orientation of plateletlike composite reinforcements has previously been observed with SiC platelets [112] and graphite platelets [113]. Chou et al [112] found that higher platelet aspect ratios lead to a higher degree of orientation. Higher orientation correlates with higher toughness as the $\mathrm{SiC}$ platelets are believed to more effectively deflect and bridge cracks when they are aligned. It was also found that the orientation of the $\mathrm{SiC}$ platelets reached a saturation point around a uniaxial pressure of $70 \mathrm{MPa}$. The GNPs in our study have a very high aspect ratio $(\sim 1500)$ and were processed using uniaxial pressures of $80-100 \mathrm{MPa}$. These factors induce the high level of GNP orientation seen in the TaC-GNP microstructure. The varying sizes of GNP enable multi-scale interactions with the TaC grains. Smaller sized GNP may not be large enough to wrap around grains and instead of hindering grain 

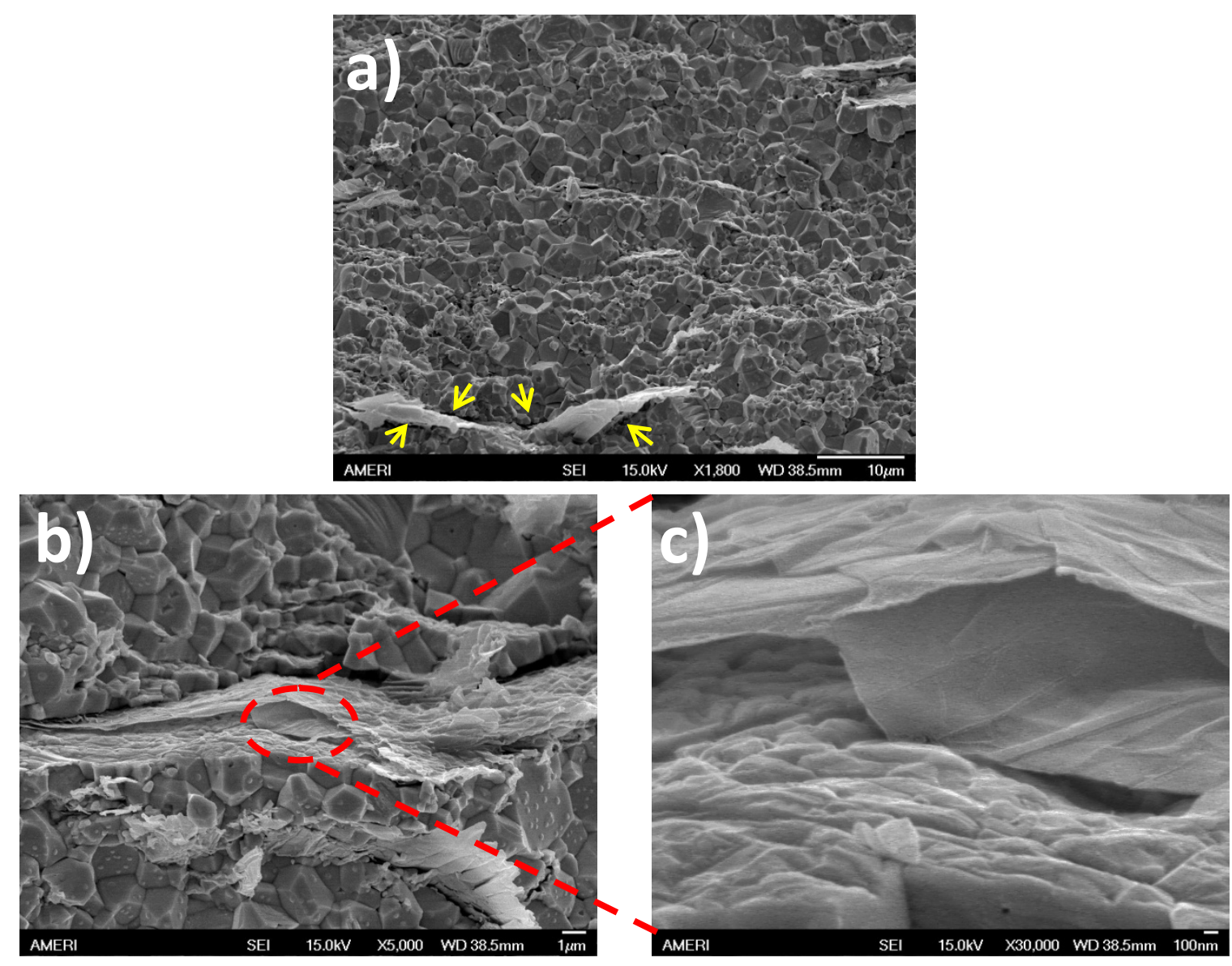

growth, they become embedded in the grains. The larger sized GNPs wrap around several grains and enable formation of continuous GNP networks throughout the microstructure.

As with the TaC-3G sample, the presence of GNP is ubiquitous in the TaC-5G structure, however the type and distribution of the GNPs varies substantially. The TaC5G microstructure displays GNPs of varying sizes however the distribution leans more heavily toward larger sized GNP. Large stacks of GNP over $20 \mu \mathrm{m}$ in lengths which were not seen in the previous samples, are present as can be seen in Fig. 4.28. It can be seen in Fig. 4.28a and $\mathrm{b}$ that the GNP/TaC interface near the larger GNPs is poor as substantial 
gaps are clearly visible. The poor interface may be due to the large amounts of wrinkles and folds in the large GNPs as shown in Fig. 4.28c. The thickness of these GNP along with the wrinkles may inhibit the GNPs ability to contour to the grain shape thus leading to larger gaps.

The presence of smaller GNPs that weave and wrap around the grains is also seen to some degree as observed in Fig. 4.29. The grain size $(1.41 \pm 0.67 \mu \mathrm{m})$ is similar to that of $\mathrm{TaC}-3 \mathrm{G}$ and smaller than that of pure $\mathrm{TaC}$. There appears to be some level of GNP networking that allows for adequate wrapping of grains and subsequent grain size inhibition. The large isolated GNPs seen in the powder likely lead to the larger GNPs seen in the consolidated structure; however, this does not appear to have an impact on the rest of the microstructure as both the densification and grain size are improved over the TaC sample. There also appears to be some limited amount of embedded GNPs such as the one shown in Fig. 4.29b. A summary of the GNP dispersion and effects on the TaCGNP composites microstructures is provided in Table 4.4.
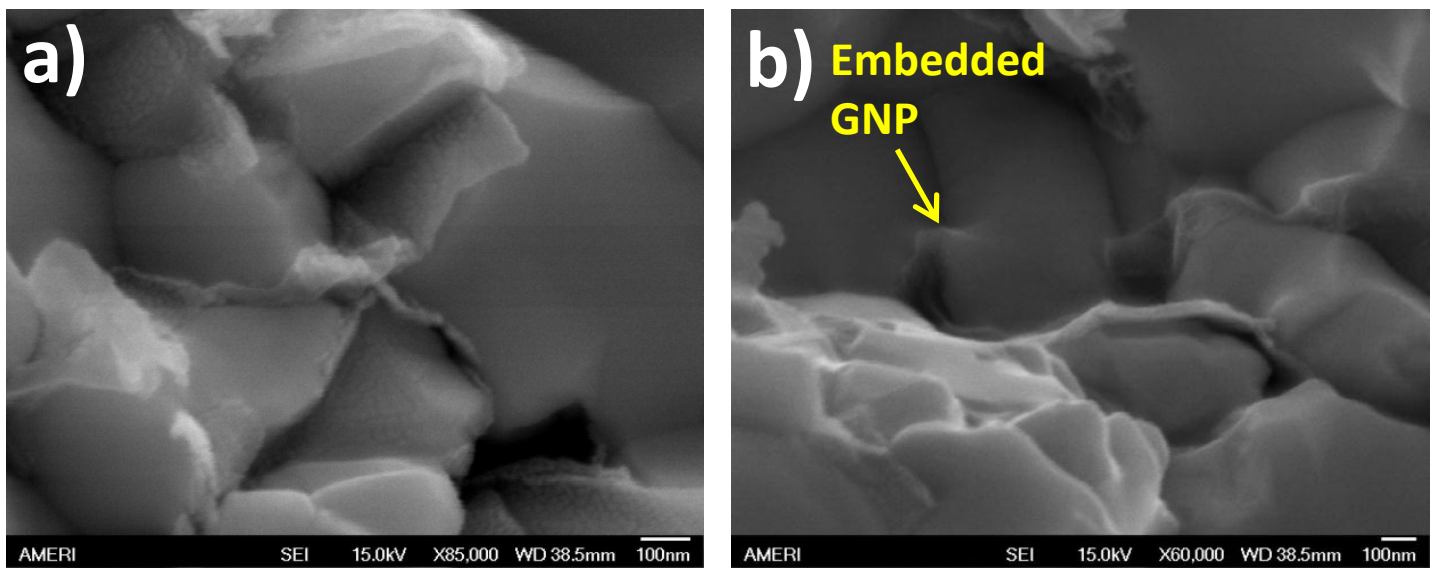


\begin{tabular}{|c|c|c|c|c|}
\hline Sample & $\begin{array}{l}\text { Grain } \\
\text { Size }\end{array}$ & GNP Dispersion & Types of GNP & $\begin{array}{l}\text { GNP Interactions } \\
\text { with } \mathrm{TaC} \text { grains }\end{array}$ \\
\hline $\mathrm{TaC}-1 \mathrm{G}$ & $3.37 \mu \mathrm{m}$ & $\begin{array}{l}\text { - GNP appear sporadically } \\
\text { throughout microstructure } \\
\text { - Consistent GNP distribution, } \\
\text { homogenous microstructure }\end{array}$ & 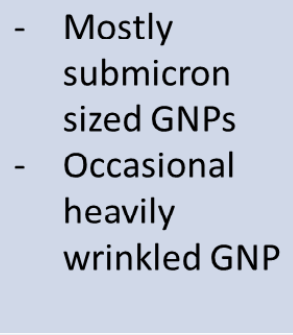 & $\begin{array}{ll}\text { - } & \text { Most GNPs } \\
\text { appear } \\
\text { embedded in } \\
\text { TaC grains } \\
\text { - } \quad \text { Heavily wrinkled } \\
\text { GNP found } \\
\text { between grains }\end{array}$ \\
\hline TaC-3G & $1.44 \mu \mathrm{m}$ & $\begin{array}{l}\text { - GNPs ubiquitous throughout } \\
\text { microstructure } \\
\text { - GNP form continuous network } \\
\text { throughout microstructure }\end{array}$ & $\begin{array}{ll}\text { - } & \text { Varying Sizes } \\
\text { - } & \text { Heavily } \\
& \text { Kinked and } \\
& \text { wrinkled GNP }\end{array}$ & $\begin{array}{ll}\text { - } & \text { Embedded GNP } \\
\text { - } & \text { Substantial } \\
\text { wrapping of TaC } \\
\text { grains }\end{array}$ \\
\hline $\mathrm{TaC}-5 \mathrm{G}$ & $1.41 \mu \mathrm{m}$ & - Uneven Distribution of GNPs & 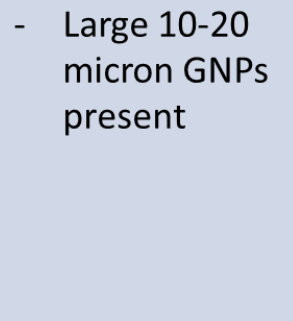 & $\begin{array}{ll}\text { - } & \text { Large GNPs have } \\
\text { weak interface } \\
\text { with TaC } \\
\text { - } & \text { Some grain } \\
& \text { wrapping } \\
\text { - } & \text { Occasional } \\
& \text { embedded GNP }\end{array}$ \\
\hline
\end{tabular}

\subsection{Phases in SPS TaC-GNP Composites}

The phases present and the structure of the graphene nanoplatelets (GNP) in sintered TaC-GNP composites are evaluated in order to gain an understanding of the effect of the extreme temperature and pressures during spark plasma sintering. The phases present in the TaC-GNP composites are evaluated by X-ray diffraction and the peak patterns are shown in Fig. 4.31. The resemblance between the peaks in the powders and the SPS compacts is clear and this confirms that no significance phase transformations have occurred as a result of the SPS processing. The formation of the undesired phases such $\mathrm{Ta}_{2} \mathrm{C}$ phase is avoided. Both the powders and the SPS samples 


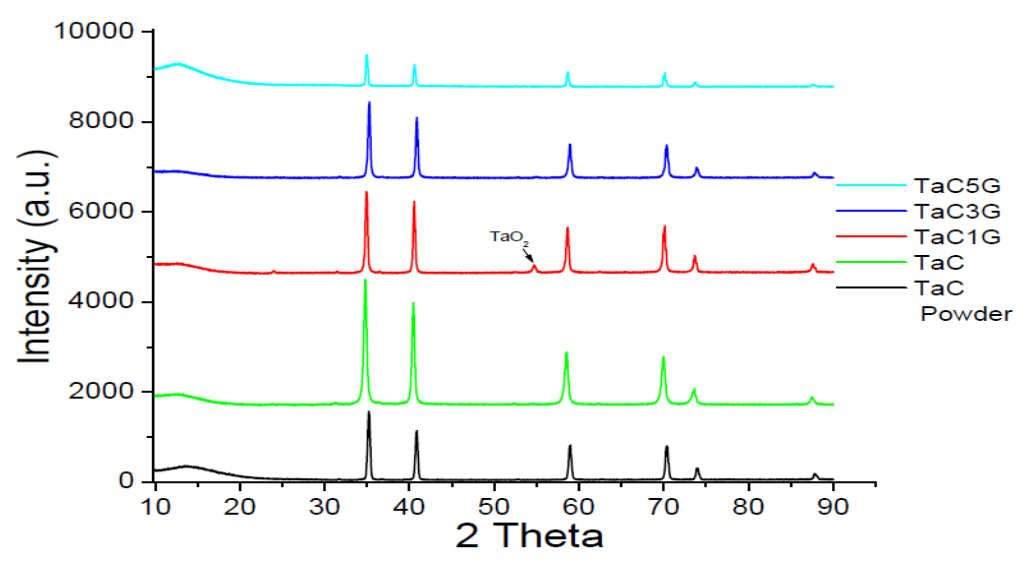

contain trace amounts of oxides such as $\mathrm{Ta}_{2} \mathrm{O}_{5}, \mathrm{TaO}_{3}$, and $\mathrm{TaO}_{2}$. There are only marginal differences in the presence of these oxides when comparing powders and SPS compacts. Only the TaC-1G contains an oxide peak $\left(\mathrm{TaO}_{2}\right)$ which is visibly larger in the SPS compact than in the powder. The relative compositions of the phases in the TaC-GNP powders and SPS compacts as determined by measuring the peak areas are shown in Table 4.5.

\begin{tabular}{|c|c|c|c|c|c|c|}
\hline \multirow[t]{2}{*}{ Powder Samples } & \multicolumn{5}{|c|}{ Composition (\%) } & \multirow[b]{2}{*}{$\underline{\text { Total }}$} \\
\hline & $\mathrm{TaC}$ & $\mathrm{Ta}_{4} \mathrm{C}_{3}$ & $\mathrm{TaO}_{2}$ & $\mathrm{TaO}_{3}$ & $\mathrm{Ta}_{2} \mathrm{O}_{5}$ & \\
\hline $\mathrm{TaC}$ & 94.06 & $6 \quad 1.25$ & 2.77 & 1.9 & 0 & 99.98 \\
\hline TaC-1G & 91.92 & $2 \quad 1.26$ & 3.29 & 3.54 & 0 & 100.01 \\
\hline TaC-2G & 92.94 & $\begin{array}{ll}4 & 1.37\end{array}$ & 2.61 & 3.09 & 0 & 100.01 \\
\hline TaC-3G & 96.76 & $\begin{array}{ll}6 & 0.58\end{array}$ & 0.35 & 2.31 & 0 & 100.00 \\
\hline TaC-4G & 95.53 & $\begin{array}{ll}3 & 0.83\end{array}$ & 1.11 & 2.53 & 0 & 100.00 \\
\hline TaC-5G & 91.8 & 1.27 & 2.2 & 4.73 & 0 & 100.00 \\
\hline \multirow[t]{2}{*}{ SPS Samples } & \multicolumn{5}{|c|}{ Composition (\%) } & \\
\hline & $\mathrm{TaC}$ & $\mathrm{Ta}_{4} \mathrm{C}_{3}$ & $\mathrm{TaO}_{2}$ & $\mathrm{TaO}_{3}$ & $\mathrm{Ta}_{2} \mathrm{O}_{5}$ & Total \\
\hline $\mathrm{TaC}$ & 95.18 & 0.92 & 0.98 & 2.92 & 0 & 100.00 \\
\hline TaC-1G & 87.46 & 1.05 & 0 & 5.81 & 5.67 & 99.99 \\
\hline TaC-2G & 91.59 & 0.93 & 2.62 & 2.89 & 1.96 & 99.99 \\
\hline TaC-3G & 89.89 & 1 & 1.86 & 5.6 & 1.65 & 100.00 \\
\hline TaC-4G & 90.48 & 1.66 & 0 & 3.26 & 4.6 & 100.00 \\
\hline TaC-5G & 90.91 & 2.86 & 1.91 & 4.33 & 0 & 100.01 \\
\hline
\end{tabular}




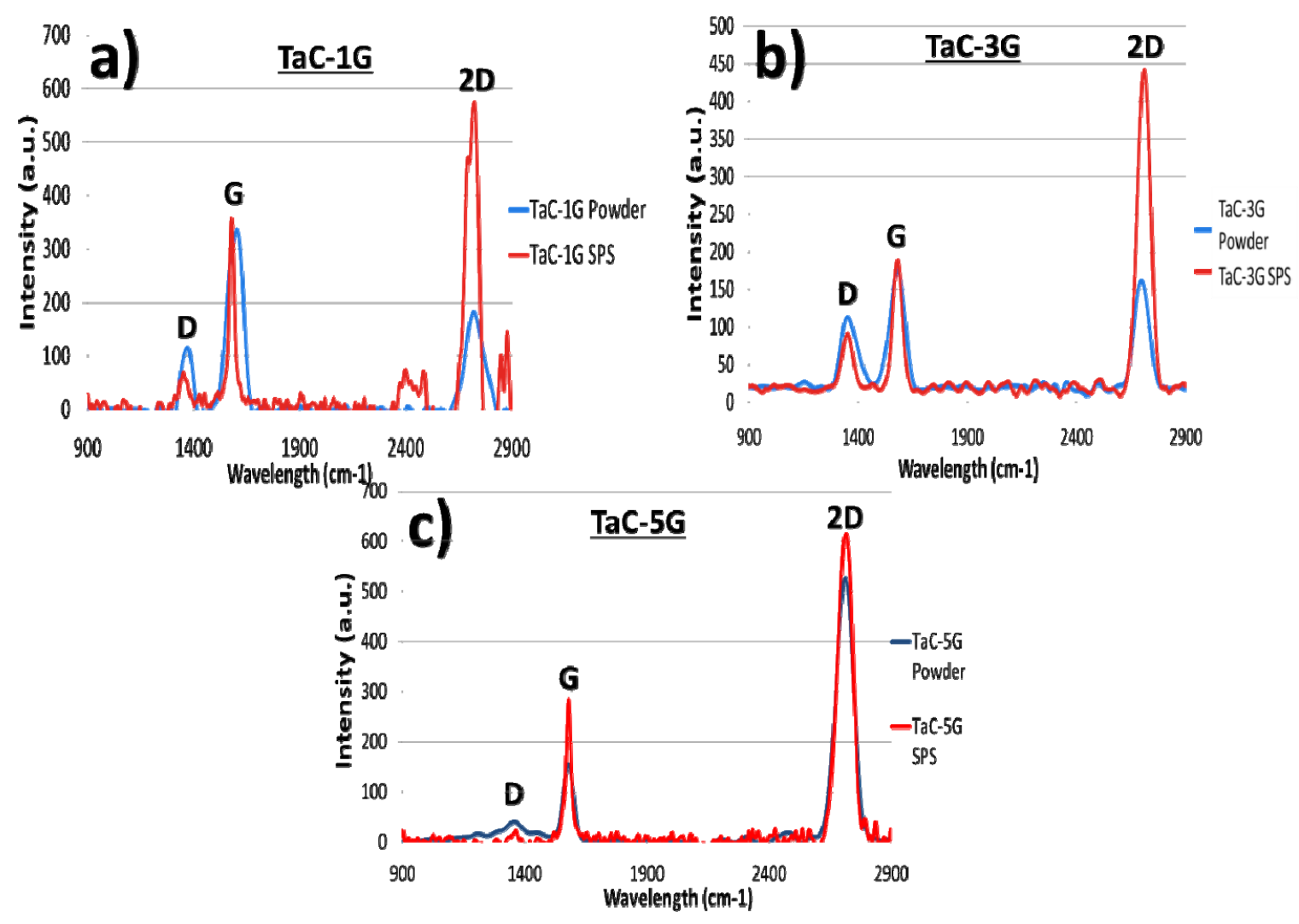

\begin{tabular}{cccccc} 
Sample & D Peak & G Peak & 2D Peak & $\mathbf{I}_{\mathbf{D}} / \mathbf{I}_{\mathbf{G}}$ & $\mathbf{I}_{\mathbf{2 D}} / \mathbf{I}_{\mathbf{G}}$ \\
\hline $\begin{array}{c}\text { 1G } \\
\text { Powder }\end{array}$ & 1370.4 & 1604.3 & 2720.6 & 0.345 & 0.543 \\
$\begin{array}{c}\text { 3G } \\
\text { Powder }\end{array}$ & 1352.02 & 1581.9 & 2697.14 & 0.603 & 0.912 \\
$\begin{array}{c}\text { 5G } \\
\text { Powder }\end{array}$ & 1357.02 & 1579.56 & 2712.22 & 0.263 & 3.401 \\
\hline 1G SPS & 1352.02 & 1579.56 & 2723.35 & 0.193 & 1.613 \\
\hline 3G SPS & 1352.02 & 1580.73 & 2709.57 & 0.416 & 2.505 \\
\hline 5G SPS & 1364.51 & 1579.56 & 2716.47 & 0.080 & 2.169 \\
\hline
\end{tabular}


The Raman spectra of the TaC-1G, TaC-3G, and TaC-5G samples are shown in Fig. 4.31 and a summary of the relevant peak ratios is provided in Table 4.6. It can be seen that the general trend is that the SPS compacts have lower $\mathrm{I}_{\mathrm{D}} / \mathrm{I}_{\mathrm{G}}$ ratios and higher $\mathrm{I}_{2 \mathrm{D}} / \mathrm{IG}$ ratios. As explained before, the $\mathrm{I}_{\mathrm{D}} / \mathrm{I}_{\mathrm{G}}$ ratio increases as the level of defects increases and the $\mathrm{I}_{2 \mathrm{D}} / \mathrm{I}_{\mathrm{G}}$ ratio decreases as the structure becomes more like graphite and less like graphene. The results obtained therefore appear peculiar at first as the SPS compacts appear to be less damaged and display a stronger graphene structure than the starting powders. The answer to these peculiar findings lie in the powder processing method and the behavior of GNP under SPS conditions.

The starting powder is dispersed through ultrasonication and this kinetic process leads to significant interactions with the harder $\mathrm{TaC}$ powders. Interactions with the $\mathrm{TaC}$ particles may generate sheet folding, wrinkling, along with defects at the GNP edges such defects were observed in the SEM micrographs. Some degree of agglomeration also occurred during powder processing. This explains the reason why the powders may have high $\mathrm{I}_{\mathrm{D}} / \mathrm{I}_{\mathrm{G}}$ ratios and low $\mathrm{I}_{2 \mathrm{D}} / \mathrm{I}_{\mathrm{G}}$ ratios, however it is not immediately clear how SPS processing reduces these defects until one takes a closer look at results of the SPS Bulk GNP samples presented earlier [148].

During SPS processing GNP undergo phenomena such as sliding and shearing off of individual GNP platelets and to a lesser degree individual graphene sheets. This shearing and sliding off may increase the amount of few layer GNP thus increasing the signature of a graphene-like structure. It can also be observed from the bulk GNP structure that large sheets of oriented GNP are observed (Fig. 4.16b). The length of these 
GNP sheets indicates that some bonding between GNP platelets may occur during SPS processing. Such bonding may also occur in the TaC-GNP composites. The bonding of platelets in the bulk GNP sample occurred edge to edge which reduces the presence of edge defects. The reduction of edge defects along with an increase in few layer GNP likely induces the reduction in the $\mathrm{I}_{\mathrm{D}} / \mathrm{I}_{\mathrm{G}}$ ratio. The increased $\mathrm{I}_{2 \mathrm{D}} / \mathrm{I}_{\mathrm{G}}$ ratios of the TaC-GNP powders and compacts are consistent with the $\mathrm{I}_{2 \mathrm{D}} / \mathrm{I}_{\mathrm{G}}$ ratios of the GNP powder and bulk GNP structure. The $\mathrm{I}_{\mathrm{D}} / \mathrm{I}_{\mathrm{G}}$ ratio of the pure GNP powder is not higher than the bulk GNP structure because it is not ultrasonicated with the hard $\mathrm{TaC}$ particles (as in the case of TaC-GNP samples) which is likely the source of most of the damage incurred by GNP during powder processing.

It should be noted that the TaC-5G sample actually experiences a higher $\mathrm{I}_{2 \mathrm{D}} / \mathrm{I}_{\mathrm{G}}$ ratio in the powder than in the SPS compact. This is likely due to the increased presence of GNP which results in agglomeration and the presence of large swaths of powder where GNP and $\mathrm{TaC}$ particles are segregated. The isolated GNP likely undergo minimal interaction with the $\mathrm{TaC}$ and retain a graphene-like structure. 


\section{Mechanical Properties of TaC-GNP Composites}

\subsection{Elastic Modulus}

The elastic modulus of the TaC-GNP samples is evaluated through nanoindentation. The reduced modulus $\left(\mathrm{E}_{\mathrm{r}}\right)$ is obtained from nanoindentation and it is related to the indented sample's elastic modulus $\left(\mathrm{E}_{\mathrm{S}}\right)$ by Eqn. (4.1).

$$
\frac{1}{E_{r}}=\frac{1-v_{i}^{2}}{E_{i}}+\frac{1-v_{S}^{2}}{E_{S}}
$$

The reduced modulus differs from the sample modulus most when the deformation of the indenter is large and this occurs when the sample being indented has a high elastic modulus. The elastic modulus and Poisson's ratio of the indenter are known to be 1140 GPa and .07 respectively, the Poisson ratio of the sample is taken to be .24 [149]. The elastic moduli measured for each sample is plotted in Fig. 4.32. The characteristic indentation curves used to generate the reduced modulus are shown in Fig. 4.33 for each

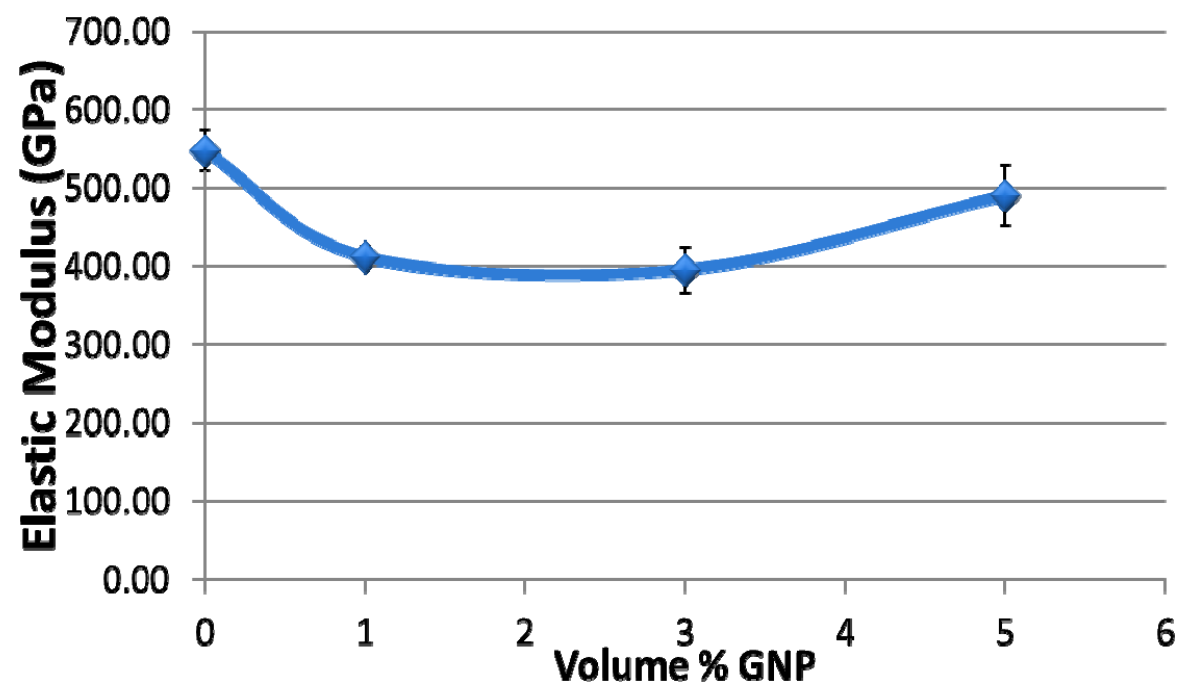




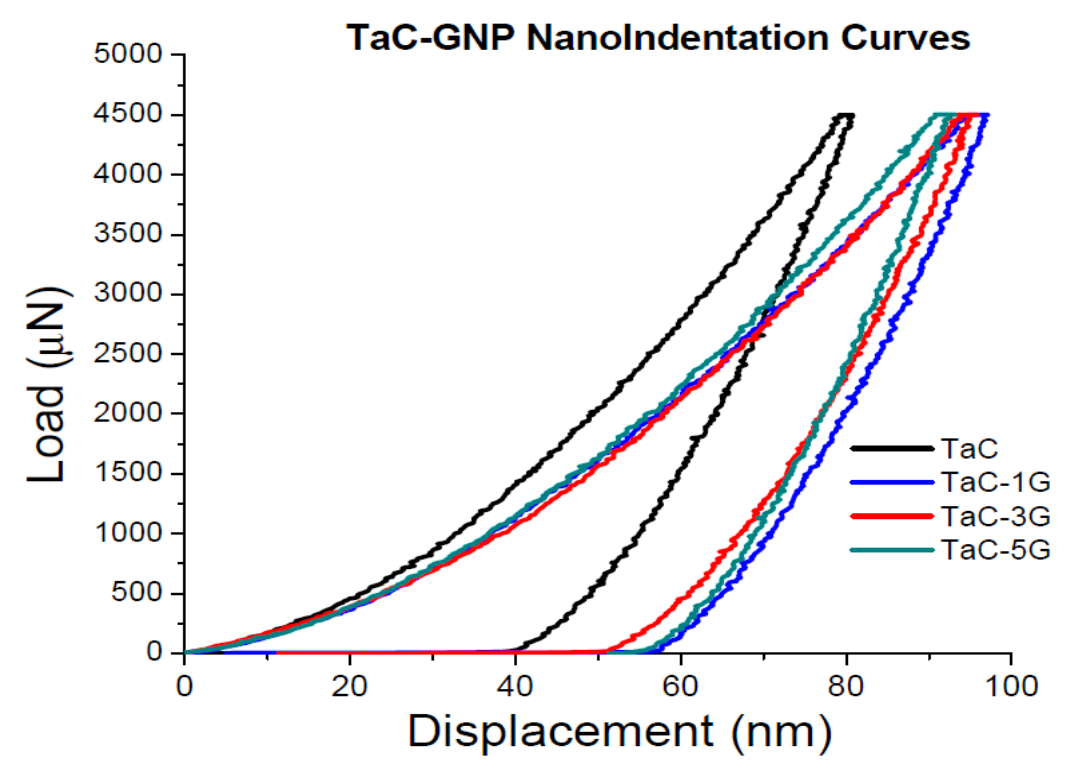

sample. A summary of nanoindentation results is provided in Table. 4.7.

In order to understand the results and trends seen in Table 4.7, it is important to emphasize the scale at which these properties were measured. Using SPM imaging, the indents made were visualized in order to quantify the size and depth of these indents. A 3-D visualization of a typical indent on the TaC-GNP samples is shown in Fig. 4.34. The width of a typical indent is approximately 1-1.5 micrometers, while the typical depths of

\begin{tabular}{|c|c|c|c|c|}
\hline Sample & Elastic Modulus & $\underline{h_{\text {MAX }}}$ & $\underline{h_{f}}$ & $\underline{\text { \% Plastic }}$ \\
\hline TaC & $\mathbf{5 4 7 . 6 6} \pm \mathbf{2 5 . 6 1}$ & 81.50 & 28.31 & $\mathbf{3 4 . 7 4}$ \\
\hline TaC-1G & $\mathbf{4 1 1 . 8 9} \pm \mathbf{1 3 . 2 2}$ & 97.44 & 50.74 & $\mathbf{5 2 . 0 8}$ \\
\hline TaC-3G & $\mathbf{3 9 5 . 3 4} \pm \mathbf{2 9 . 6 7}$ & 94.04 & 47.73 & $\mathbf{5 0 . 7 6}$ \\
\hline TaC-5G & $\mathbf{4 9 0 . 4 3} \pm \mathbf{3 8 . 8 0}$ & 92.99 & 54.02 & $\mathbf{5 8 . 0 9}$ \\
\hline
\end{tabular}


the indents are about $40 \mathrm{~nm}$ for $\mathrm{TaC}$, and about $60 \mathrm{~nm}$ for the TaC-GNP composites. The depth obtained during SPM is the depth after recovery. Fig. 4.33 shows the TaC sample typically had post-recovery depths of $\sim 40 \mathrm{~nm}$ while the TaC-GNP composites typically had depths of $\sim 50-60 \mathrm{~nm}$. The width of the indents is especially critical as it reveals that the indent size is of the order of magnitude of the grain sizes. The nanoindentation experiments thus give information about properties obtained from a single grain. The values can also be heavily influenced by location, as indents done in between grains will have substantially different (lower) properties than those done on a single grain.

Elastic modulus values obtained for the TaC sample $(547.7 \pm 25.6)$ approach the upper spectrum of values in the literature [149]. Such literature values are typically obtained through experiments and simulations from single crystals of TaC. Our values are largely obtained from single grains and thus they are representative of the intrinsic properties of $\mathrm{TaC}-$ the effects of microstructure are minimal here. The TaC-GNP

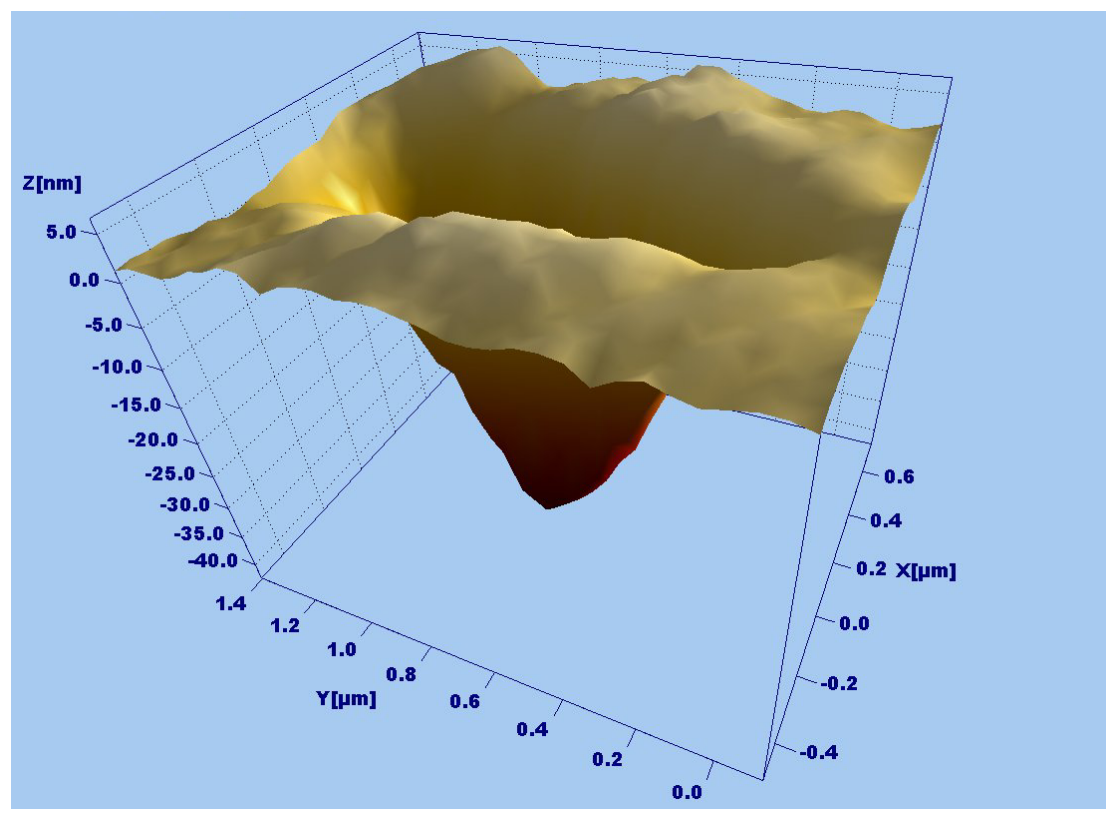


samples have different values because the GNP play a role when the TaC grain being indented is surrounded by platelets. An individual grain is held in position by the grains under and adjacent to it. In the case of $\mathrm{TaC}$, the grain being indented will be well supported by the surrounding, tightly bound grains. In the TaC-GNP samples however, there can be GNP adjacent and underneath the grain being indented. These GNP will cause increased displacement of the grain in two modes.

One is that GNP in between $\mathrm{TaC}$ grains will act as a lubricating phase between grains hence allowing slippage of the grain. The bonds between individual graphene sheets in GNP are held by weak Van der Waals forces; when a shearing force is applied by the grains, sheets of graphene will shear off from the GNP causing the $\mathrm{TaC}$ grain to slip and be further displaced. The other mode in which GNP may increase displacement of a $\mathrm{TaC}$ grain is if a grain is sitting on top a GNP. The GNP will not support the TaC grain as would another TaC grain, instead the GNP will deform and shear off as shown in Fig. $4.35 \mathrm{a}$. Fig. $4.35 \mathrm{~b}$ clearly shows that some areas where GNPs are present have
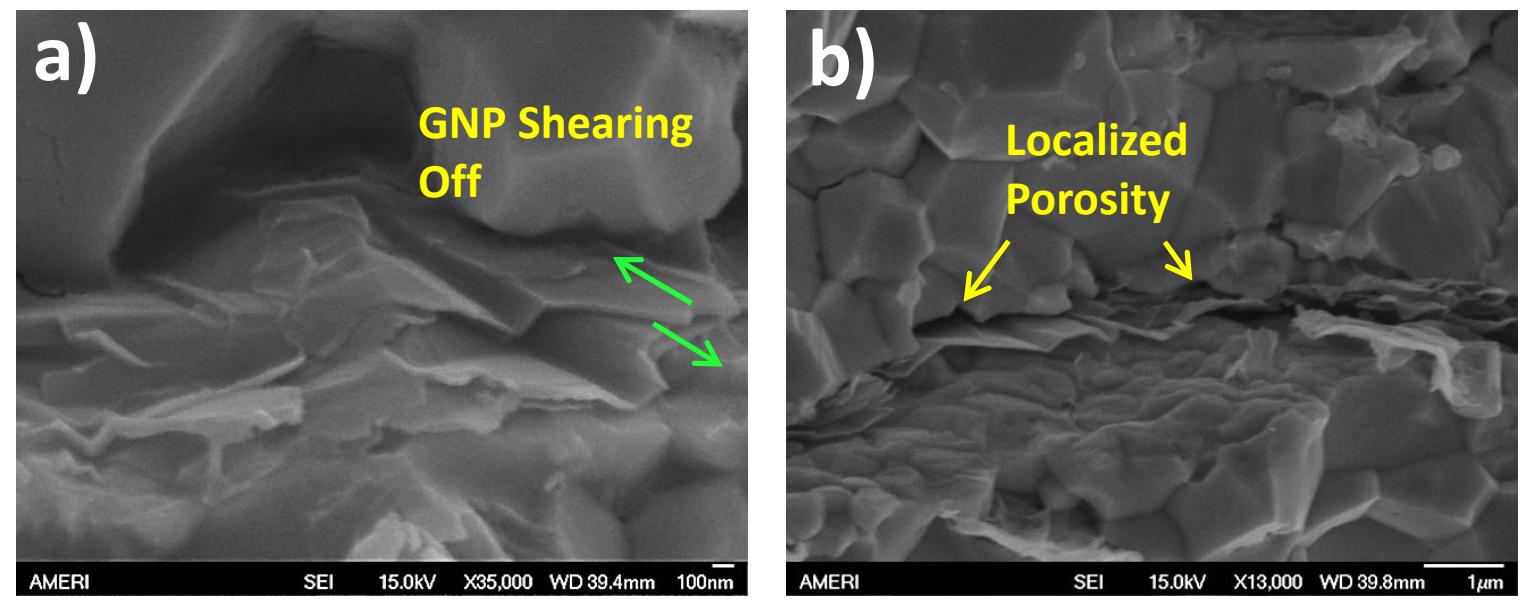
localized porosity between grains which provides space for the GNP to deform and the TaC grain to be displaced.

Elastic modulus measurements of TaC-GNP samples are therefore influenced by GNP. For a composite material it is desirable to take into account the effect of the secondary phase which is being accomplished here. The elastic moduli of the TaC-GNP samples are very similar in spite of the varying amounts of GNP. The TaC-1G and TaC3G samples have elastic moduli values that differ slightly, however it is within the margin of error. The TaC-5G sample has elastic modulus values which are higher than the other TaC-GNP samples for two reasons. The TaC-5GNP sample exhibited a distribution of GNP that was poorer than in the other two samples. Many regions in the TaC-5GNP sample were observed to have no GNP and hence some of the indents may have occurred on areas without GNP. This variation in microstructure leads to a larger variation in properties; low values reflect high GNP areas, while high values reflect GNP depleted areas - this resulted in the TaC-5G having the highest error bar of any sample. The TaC-5G sample also had the highest densification, so local porosity effects induced by the GNP would be suppressed.

\subsection{Hardness}

The hardness of the TaC-GNP samples was measured at different scales and different loads. The nanoindentation results are discussed first, these values were obtained from the same indentations used to calculate the elastic modulus. The nano- 


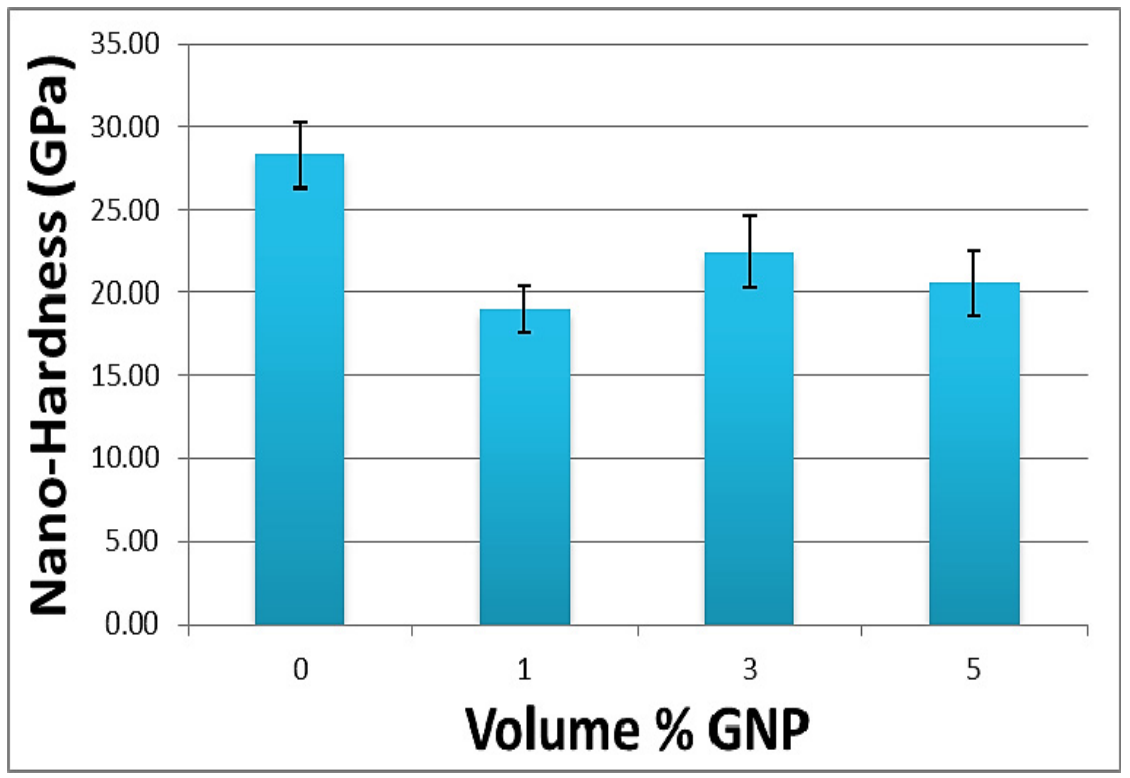

hardness results are shown in Fig. 4.36. The general trend is that the TaC sample exhibits a much higher hardness than the TaC-GNP composites. The reasoning here is much the same as for the elastic modulus - when indenting on a single TaC grain, GNP will not support the load as efficiently as another hard $\mathrm{TaC}$ grain would. Fig. 4.37 schematically illustrates how GNP can have a drastic effect when indenting on a single grain as compared to an indentation spanning many ( $>30)$ grains. A GNP underneath a TaC grain being indented will plastically deform much more than the TaC grain as GNPs are a soft phase in the out of plane direction. A TaC grain that slips as a result of GNP shearing off will also be deflected permanently. These are the same mechanisms which result in a lower elastic modulus being obtained for the TaC-GNP samples.

The slight variations in nano-hardness in the TaC-GNP samples can be attributed to the amount of GNP along with the grains sizes. The TaC-3G sample has a slightly higher hardness than the TaC-1G sample and this is due to the lower grain size in the 
TaC-3G sample. A finer grain structure gives less room for GNP to accumulate between boundaries, and therefore there will be less GNP between the grain being indented and the grain supporting it. This effect is offset in part, by the increased GNP content which gives a greater likelihood of finding a $\mathrm{TaC}$ grain surrounded and supported by GNP instead of $\mathrm{TaC}$ grains. The decreased grain size effect is canceled out by the increasing amount of GNP in the TaC-5G sample as this sample has nearly the same hardness as the TaC-1G sample. It should be noted that the densification of the TaC-GNP samples was higher than that of the $\mathrm{TaC}$ sample, it is conceivable that the difference in hardness between the TaC samples and the TaC-GNP samples would be greater if the densification was the same. If the TaC-GNP samples had the same densification as $\mathrm{TaC}$, then it is likely the indented grains would have more empty space along with GNP around them, thus lowering both the hardness and elastic modulus values.

Microindentation testing was done on the samples using loads of $5 \mathrm{~N}, 20 \mathrm{~N}$, and

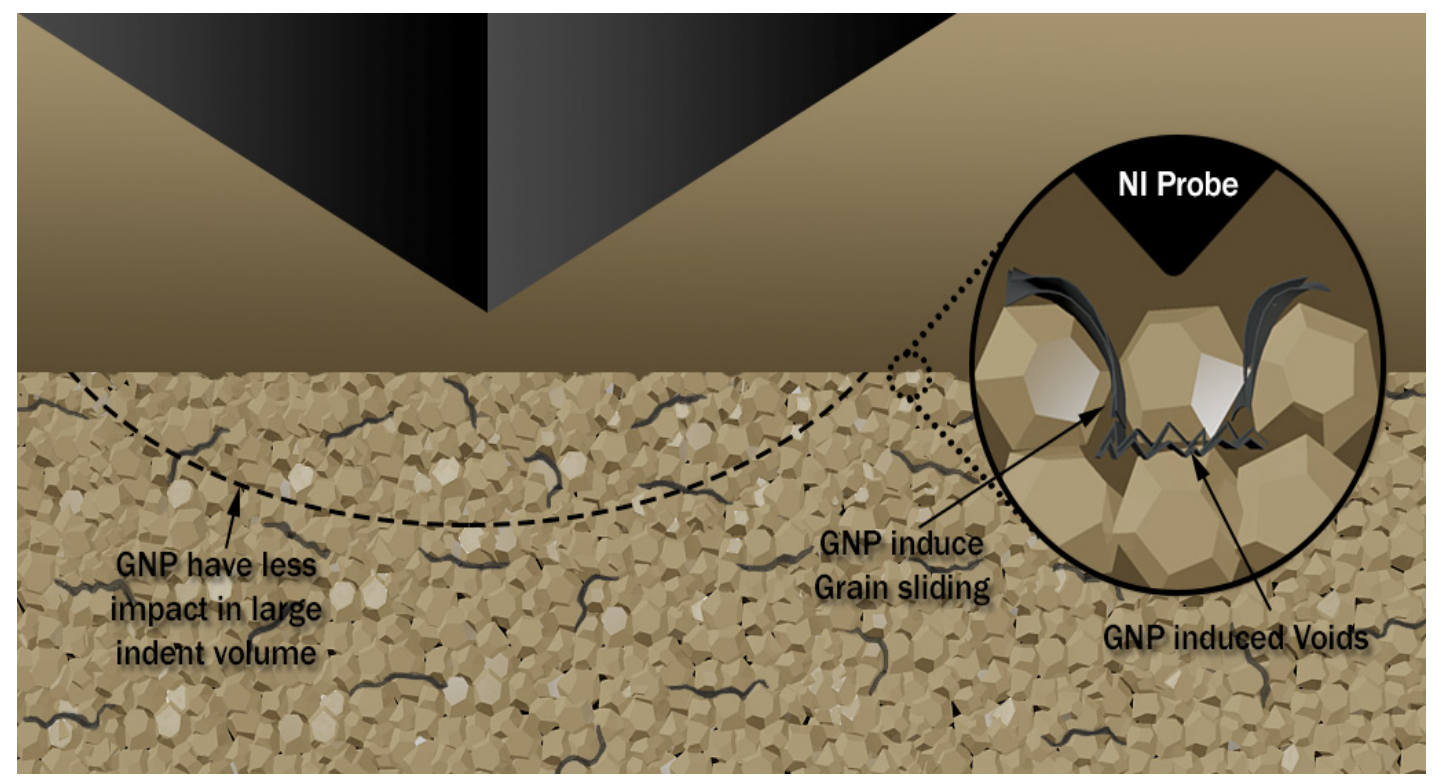


$30 \mathrm{~N}$. The results at multiple loads are shown in Fig. 4.38. A larger volume than during nanoindentation is indented so the $\mathrm{TaC}$ matrix should have greater influence, especially at low GNP concentrations. Throughout all loads, it is seen that the hardness decreases with increasing GNP content - which is expected as GNP have lower hardness than TaC. The effect of GNP is reduced both at lower GNP concentrations and at lower loads.

When indented using a $5 \mathrm{~N}$ load, the $\mathrm{TaC}$ sample and the $\mathrm{TaC}-1 \mathrm{G}$ sample have virtually the same values. This indicates that at low loads a minimal presence of GNP is not detrimental to the hardness of the TaC-GNP composite. At higher loads, $20 \mathrm{~N}$ and 30 $\mathrm{N}$, the TaC-1G samples has a hardness that is lower than the $\mathrm{TaC}$ sample by $\sim 6.8 \%$ and $\sim 16.5 \%$ respectively. This increased rate of weakening with higher load is seen in the TaC-3G and TaC-5G samples as well. For loads of 5N, $20 \mathrm{~N}$, and $30 \mathrm{~N}$, the hardness of

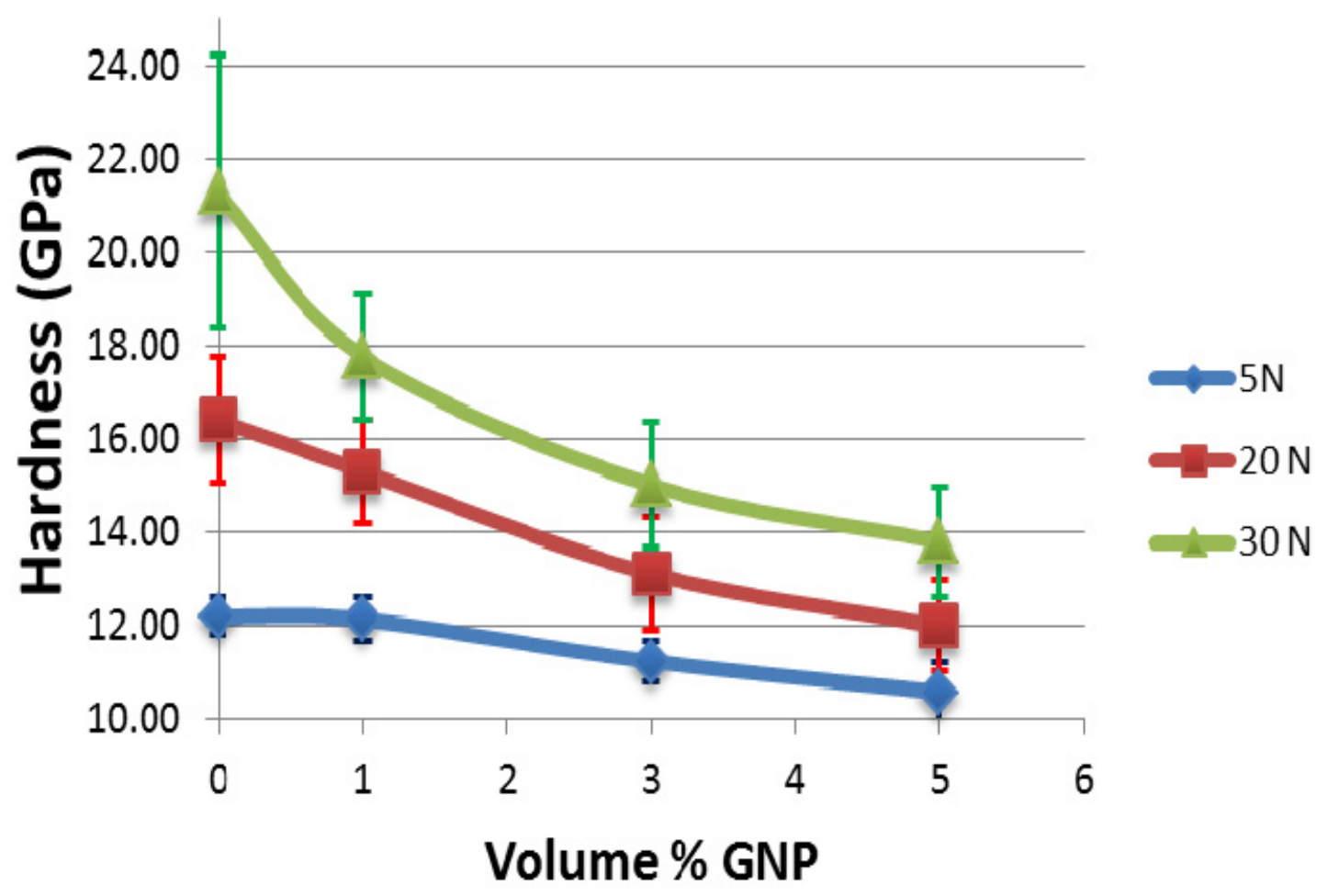


TaC-3G decreases by $\sim 7.9 \%, \sim 20.1 \%$, and $\sim 29.5 \%$ respectively. Similarly, for the TaC5 G sample the decrease is $\sim 13.3 \%, \sim 27 \%$, and $\sim 35.3 \%$ respectively. The GNP appear to have a greater influence at higher loads, possibly because as a greater volume is indented, the accumulation of deformed GNP lead to an instability in the $\mathrm{TaC}$ structure which causes a larger amount of plastic deformation.

A trend in the data that is not focused on here, but is surprising, is the overall increase in hardness at higher loads. This trend is not expected and it is likely related to the $\mathrm{TaC}$ structure. It may be possible that at higher loads, the plastic deformation induces some localized consolidation of the $\mathrm{TaC}$ structure which results in higher resistance to further plastic deformation. As seen in Fig. 4.38, the TaC sample has the greatest increase in measured hardness as the indentation is increased from $5 \mathrm{~N}$ to $30 \mathrm{~N}$. The TaC sample has the lowest densification of all the samples, and thus it may have benefitted the most from any localized consolidation effect. In contrast, the TaC-5G sample had the highest densification of any sample, and it experienced the lowest increase in measured hardness as the indentation load was increased from $5 \mathrm{~N}$ to $30 \mathrm{~N}$.

\subsection{Fracture Toughness}

The fracture toughness of the TaC-GNP composites is evaluated by measuring the cracks generated by the $20 \mathrm{~N}$ and $30 \mathrm{~N}$ indentations, and then employing the Anstis Equation (Eqn. 3.1). The fracture toughness results are presented in Fig. 4.39 and it can be seen that the GNP enhance the fracture toughness of the TaC-GNP composites. The 
increase in fracture toughness is largest in the TaC-5G sample ( $\sim 99 \%)$, followed by the TaC-1G sample ( 48\%), and TaC-3G sample ( $\sim 39 \%)$. Fig. 4.40 presents optical images of indents on the $\mathrm{TaC}$ and $\mathrm{TaC}-5 \mathrm{G}$ samples in order to qualitatively show the increase in toughness in the TaC-5G sample as evident by the suppression of cracks. As emphasized in Chapter 1 and 2, one of the main objectives of adding GNP was to increase the fracture toughness, as $\mathrm{TaC}$ and ceramics in general are very brittle.

The fracture toughness of ceramics has been increased before by up to $235 \%$ when adding GNP by Walker et al [107], so our results here are in line with previous results in the ceramic community. In this study, fundamental toughening mechanisms of GNP (Section 5.4.1) are examined by synthesizing a bulk GNP sample. This sample had no potential extrinsic matrix effects and thus the behavior observed is inherent to GNP. The actual mechanisms observed in the fracture surface and cracks of the TaC-GNP samples (Section 5.4.2) are also examined in the forthcoming sections

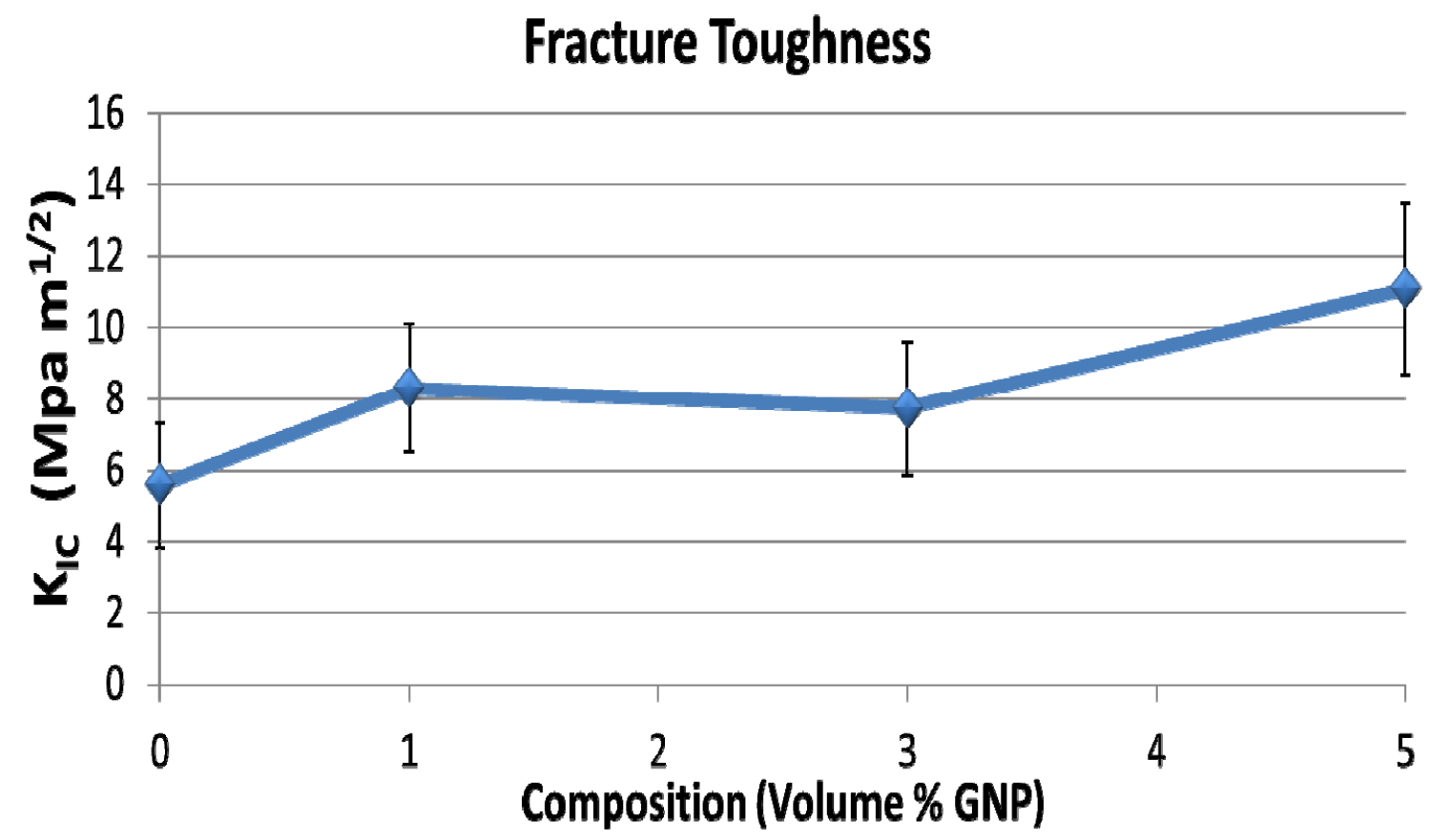



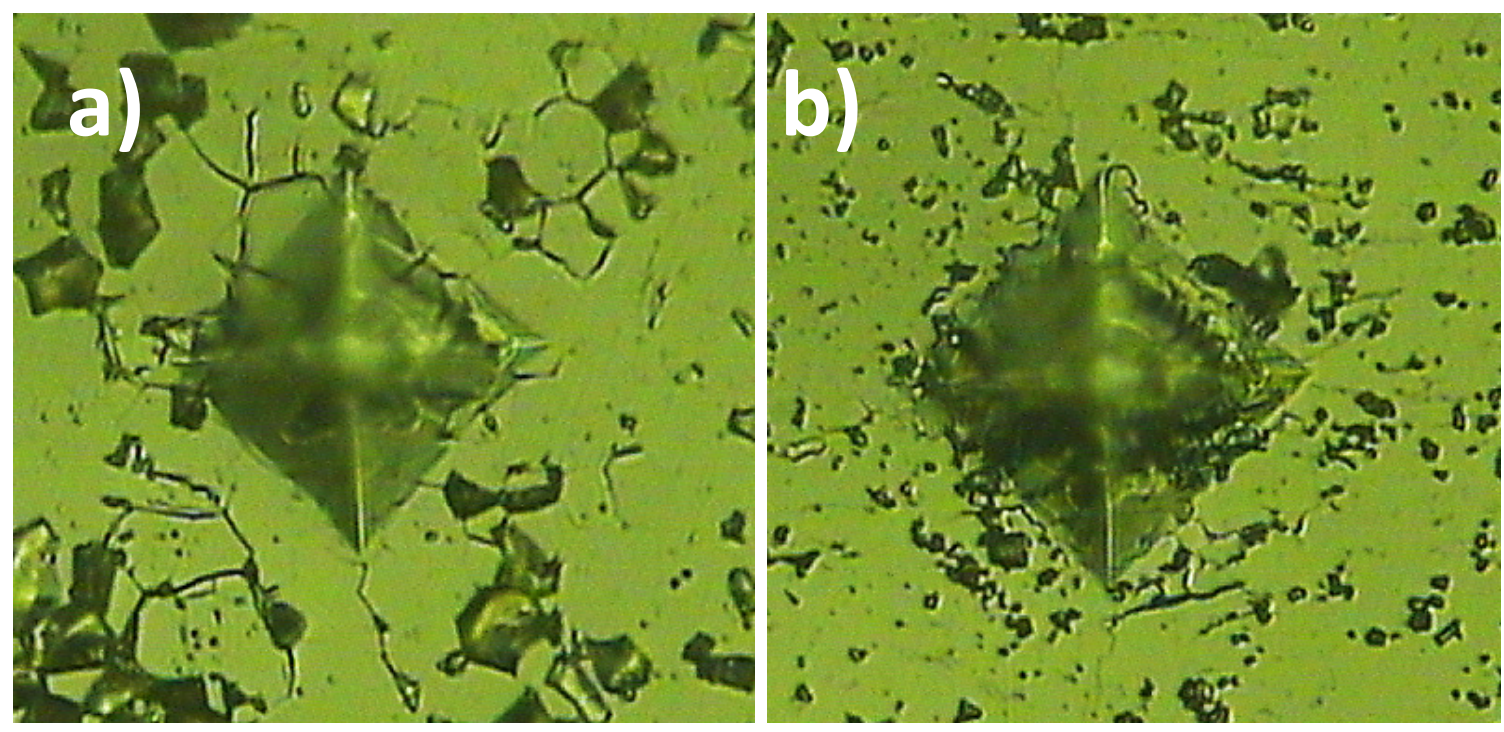

\subsection{Toughening Mechanisms}

\subsubsection{Toughening Mechanisms in Bulk GNP}

Fig. 4.41 shows SEM images of the fracture surface of GNPs revealing unique bending, folding and curling structure leading to energy dissipation. The bending of the GNPs is observed to occur for a single stack( Fig. 4.41 b) and multiple stacks as observed in Fig 4.41a and d. These bends are the result of the high pressure experienced during the SPS processing. Fig. 4.40c shows wrapping phenomena of GNPs with minimal damage on the surface. It can be concluded from Fig. $4.41 \mathrm{~d}$ that despite bending angles exceeding 90 degrees and in some cases approaching 180 degrees, the GNP does not fracture, giving way to the U-shape structure. GNP layers are concurrently undergoing sliding during the bending, especially between stacks of GNP as in Fig. 4.41d. Some of the stacks become offset from the other stacks during the bending. 
This form of deformation is similar to the pseudo-plastic behavior observed in $\mathrm{Ti}_{3} \mathrm{SiC}_{2}[151,152]$. Mechanisms in the $\mathrm{Ti}_{3} \mathrm{SiC}_{2}$ microstructure include interlayer sliding, grain buckling, and kink band formation. The kink boundaries are believed to enhance mechanical properties by containing damage. The bending observed in the GNP occurs at much larger angles than those in the $\mathrm{Ti}_{3} \mathrm{SiC}_{2}$ kink band formation and would be expected to have the same toughening effects as the $\mathrm{Ti}_{3} \mathrm{SiC}_{2}$. Since these sliding and bending mechanisms are observed in a pure bulk GNP structure and not a composite, it can be concluded that this is a fundamental energy dissipation mechanism of GNP.
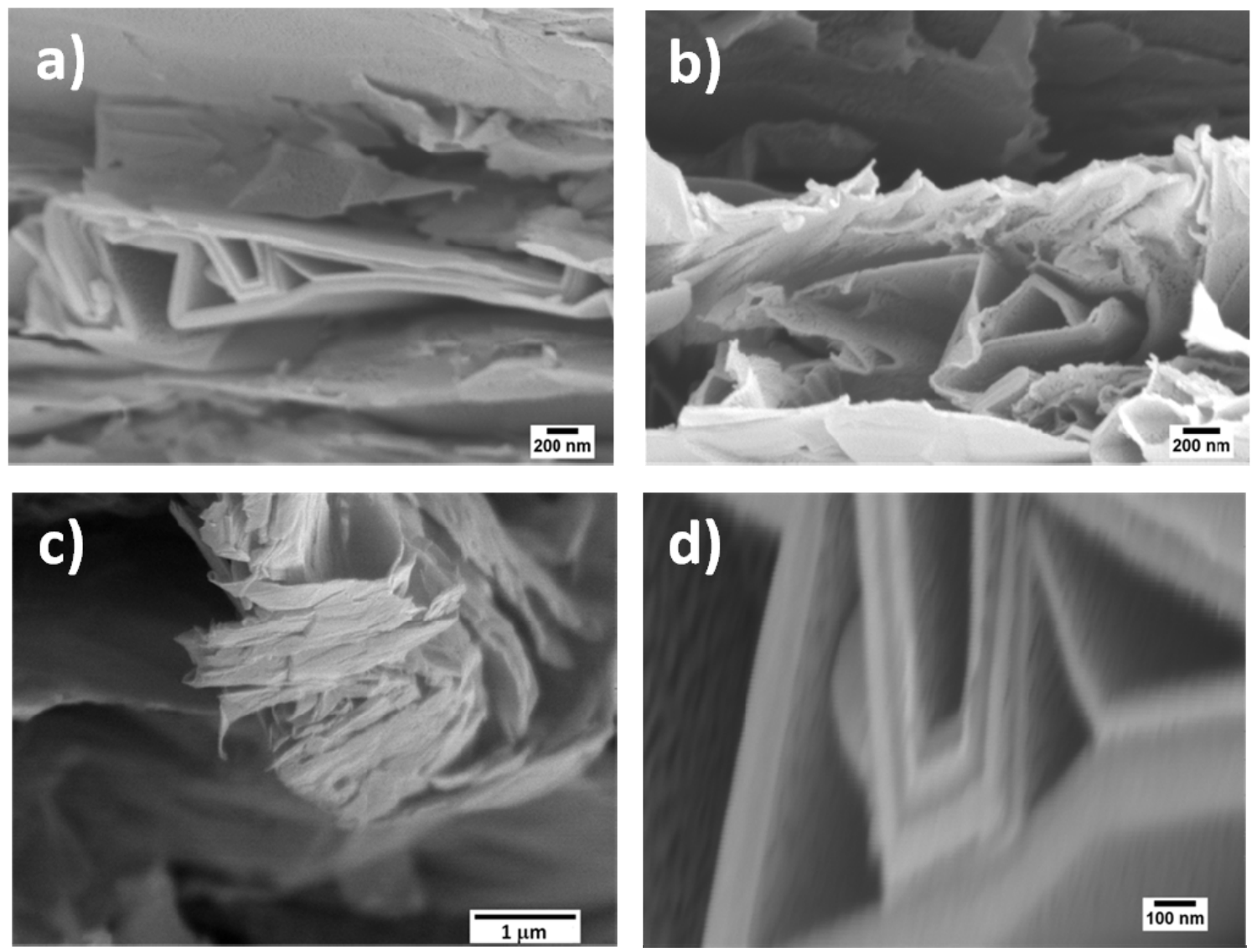
Hence, GNPs can offer unique energy dissipating mechanisms when used as reinforcement in ceramic matrix composites, as energy would be absorbed during the bending and sliding of the GNPs. This energy dissipation mechanism would presumably not only occur during processing but also during load bearing. The toughening mechanisms and the ability of the GNP to absorb energy during load bearing make it an excellent candidate for use in brittle ceramic matrices. As is the case with $\mathrm{Ti}_{3} \mathrm{SiC}_{2}$, the GNP bends would be expected to contain the spread of damage in a composite. This bending mechanism is likely responsible for the wrapping phenomena observed by Walker [107] where the GNPs bend and wrap themselves around grains thus inhibiting crack propagation and increasing toughness.

\subsubsection{Toughening in TaC-GNP Composites}

The TaC-GNP samples display the type of toughening mechanisms observed in the bulk GNP samples, as well those observed in the GNP reinforced composites literature [104-109]. Fig. 4.42 presents some of the GNP mechanisms observed in the TaC-GNP samples that were proven to be inherent GNP mechanisms in the previous section. Fig. 4.42a and $\mathrm{b}$ shows highly kinked and corrugated GNP embedded in multiple $\mathrm{TaC}$ grains. It can be seen that there are two distinct stacks of GNP laying on top each other. As discussed before, the kinking and sliding of GNP toughens the matrix by dissipating energy that would otherwise contribute to crack propagation. The GNP in Fig. $4.42 \mathrm{c}$ is undergoing sheet sliding thus dissipating energy from shear stresses. As in the bulk GNP, all the kinked GNPs show no signs of fracturing despite the high angles of bending. 
Another known GNP mechanism occurs when GNP interacts with the matrix wrapping of GNP around grains. This provides a toughening effect as well and a grain inhibiting mechanism as discussed earlier. A grain that may have been wrapped around two grains is shown in Fig. 4.43b; the top surface of the GNP is seen near the surface of two grains indicting that the GNP wrapped and not embedded. The wrapped GNP is spanning across a propagating crack indicating that the GNP is attempting to resist the tensile force separating the two grains apart. The inset shows how GNP wrapping can
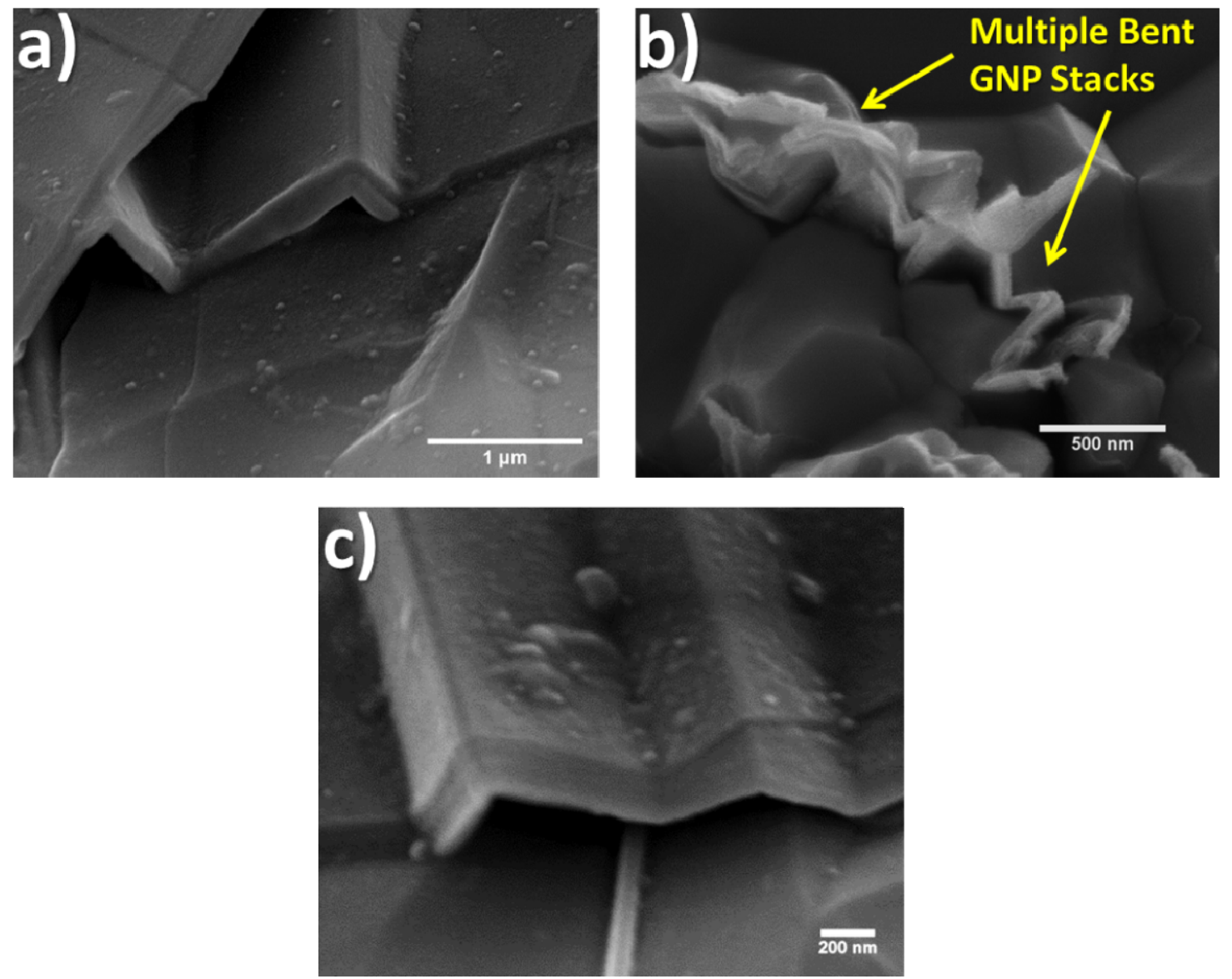
occur across multiple grains. Oftentimes the GNP will become embedded in grains thus holding them together. Energy is dissipated during loading when the GNP is pulled out prior to the grains yielding. Fig. 4.43a shows an instance of GNP pullout, the GNP is only embedded in one grain demonstrating that the crack caused it to be pulled out from the grain. This pulling out of GNP also dissipates energy otherwise used for crack propagation hence toughening the ceramic matrix.

The GNP mechanisms discussed above can lead to the inhibition of crack propagation. GNP can be seen to deflect a propagating crack in Fig. 4.44a. Both single and multiple-platelet GNP bridge cracks by attempting to bind grains together. Fig. 4.44 displays varying levels of GNP crack bridging observed in cracks generated by $90 \mathrm{~N}$ ind entations. The amount of GNP bridging is extensive as seen in Fig. 4.44b-d, however the structure of the GNP is diverse. The GNP in Fig. 4.44b appears to consist of a single GNP in contrast to those in Fig. 4.44d which consist of several overlapping GNP. GNP in Fig. 4.44b and c appear to be twisted; this indicates that the GNP has been opposing not
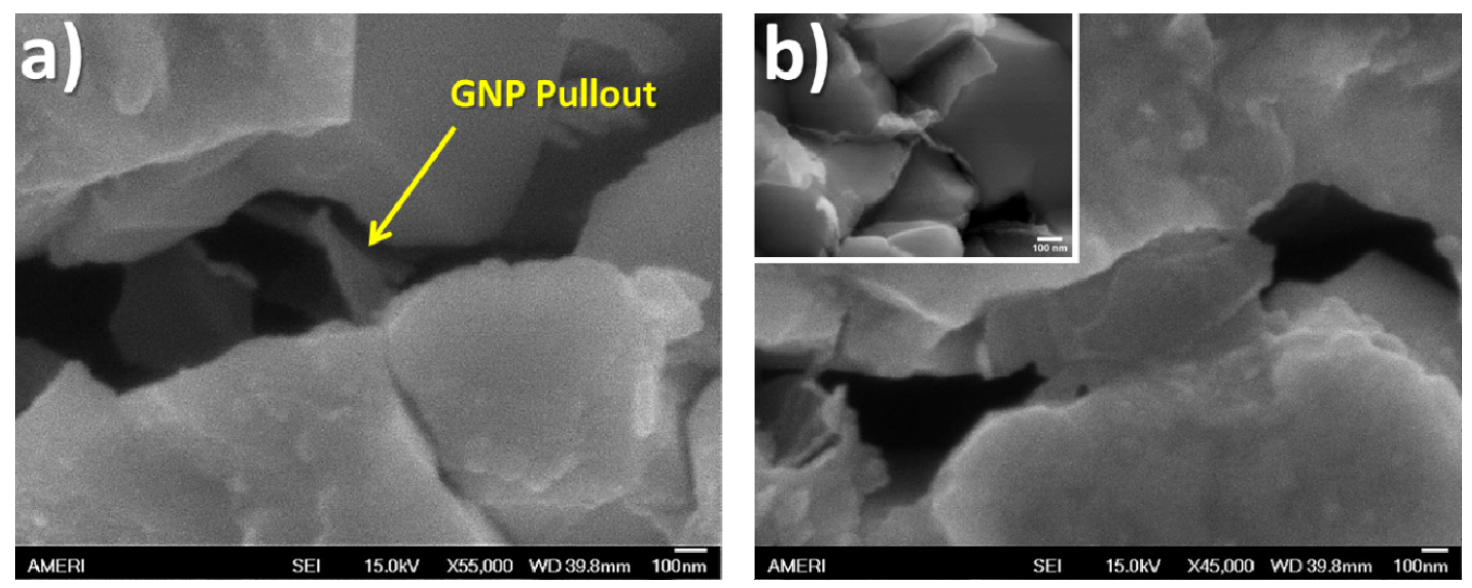
only tensile stresses, but also some degree of torsion. The GNP can also be seen to act deep inside the crack as shown in Fig. 4.44c. The improvement in fracture toughness of the TaC-GNP samples vindicates that the microstructural mechanisms observed and discussed above, do indeed have a toughening effect on the matrix.

From the above discussion, it is evident that the GNP toughening mechanisms have multi-scale effects. The fundamental intrinsic mechanisms of GNP are sheet sliding and GNP bending and kinking as they are observed in the bulk GNP. In a composite, the GNP interacts with the matrix leading to grain wrapping and sheet pullout. Together the intrinsic GNP mechanisms and their interactions with grains leads to crack inhibiting mechanisms - crack bridging and crack deflection.
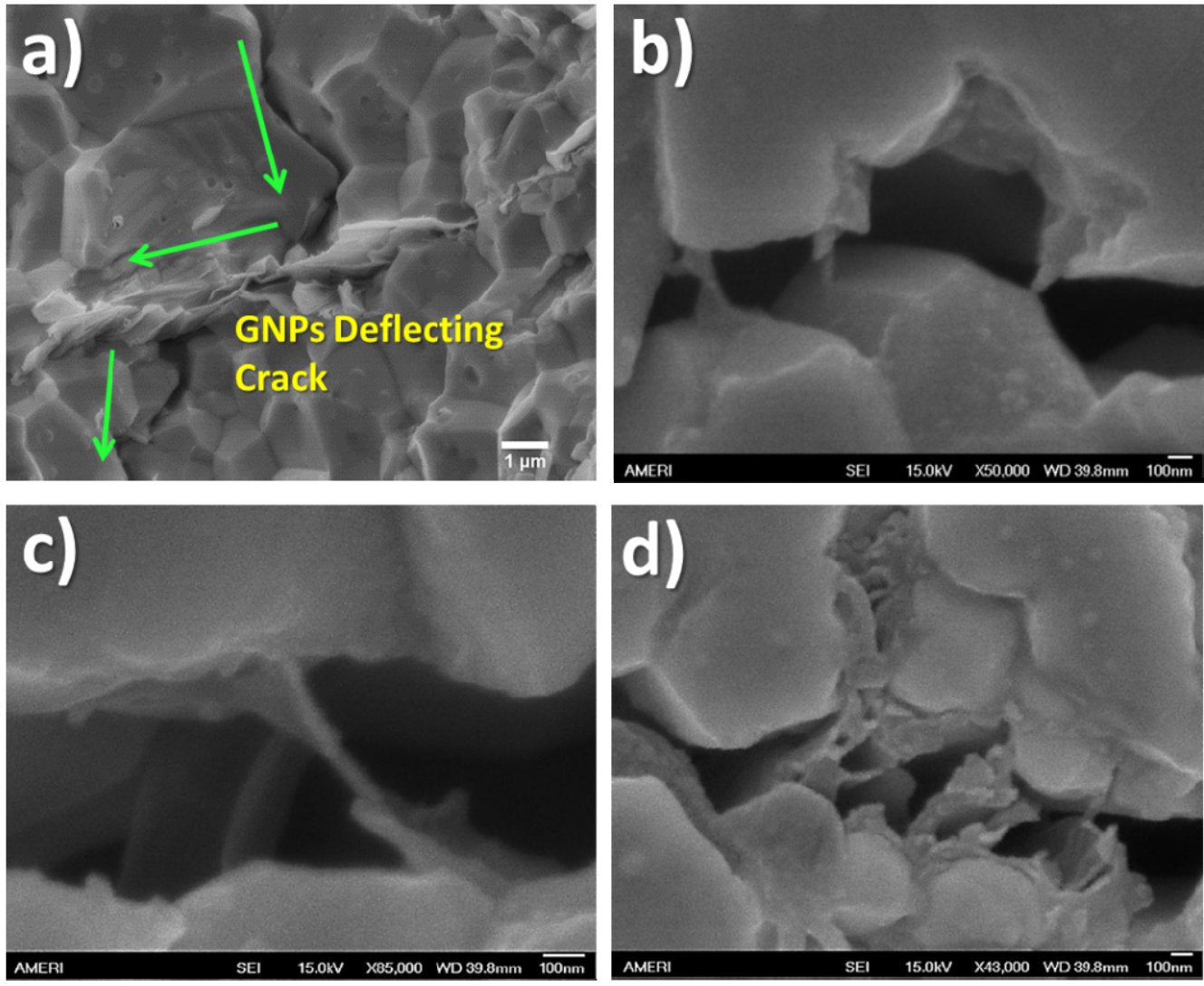


\section{TaC-GNP Oxidation Behavior}

UHTCs are intended for high temperature aerospace applications and therefore the last crucial piece in evaluating the effectiveness of GNP reinforcement for $\mathrm{TaC}$ composites is to investigate the performance of TaC-GNP composites in a high temperature plasma flow. The properties of the plasma flow were discussed in Chapter 3 . The importance of this type of oxidation test is emphasized here again - UHTC materials are expected to perform in ionized high temperature environments and thus the oxidation behavior under these types of conditions is of greater value than conventional furnace oxidation tests.

\subsection{Oxidation of TaC-GNP Composites}

Images of the oxidized TaC-GNP composites are shown in Fig. 4.45 and it can be seen that samples survived the extreme environment. The composites have been heavily oxidized and are thoroughly covered with a white oxide layer. The high temperature plasma flow results in an oxide layer with a variety of oxide phases. The phases were identified using XRD, patterns are shown in Fig. 4.46 and a breakdown of the composition as compared to the starting SPS samples is provided in Table 4.8 . Compositions were determined by measuring the area under the peaks for each phase. The major phase in the oxide structure is Tantalum Pentoxide $\left(\mathrm{Ta}_{2} \mathrm{O}_{5}\right)$. The reaction governing this phase change is given below:

$$
2 \mathrm{TaC}+\frac{9}{2} \mathrm{O}_{2} \rightarrow \mathrm{Ta}_{2} \mathrm{O}_{5}+2 \mathrm{CO}_{2}
$$



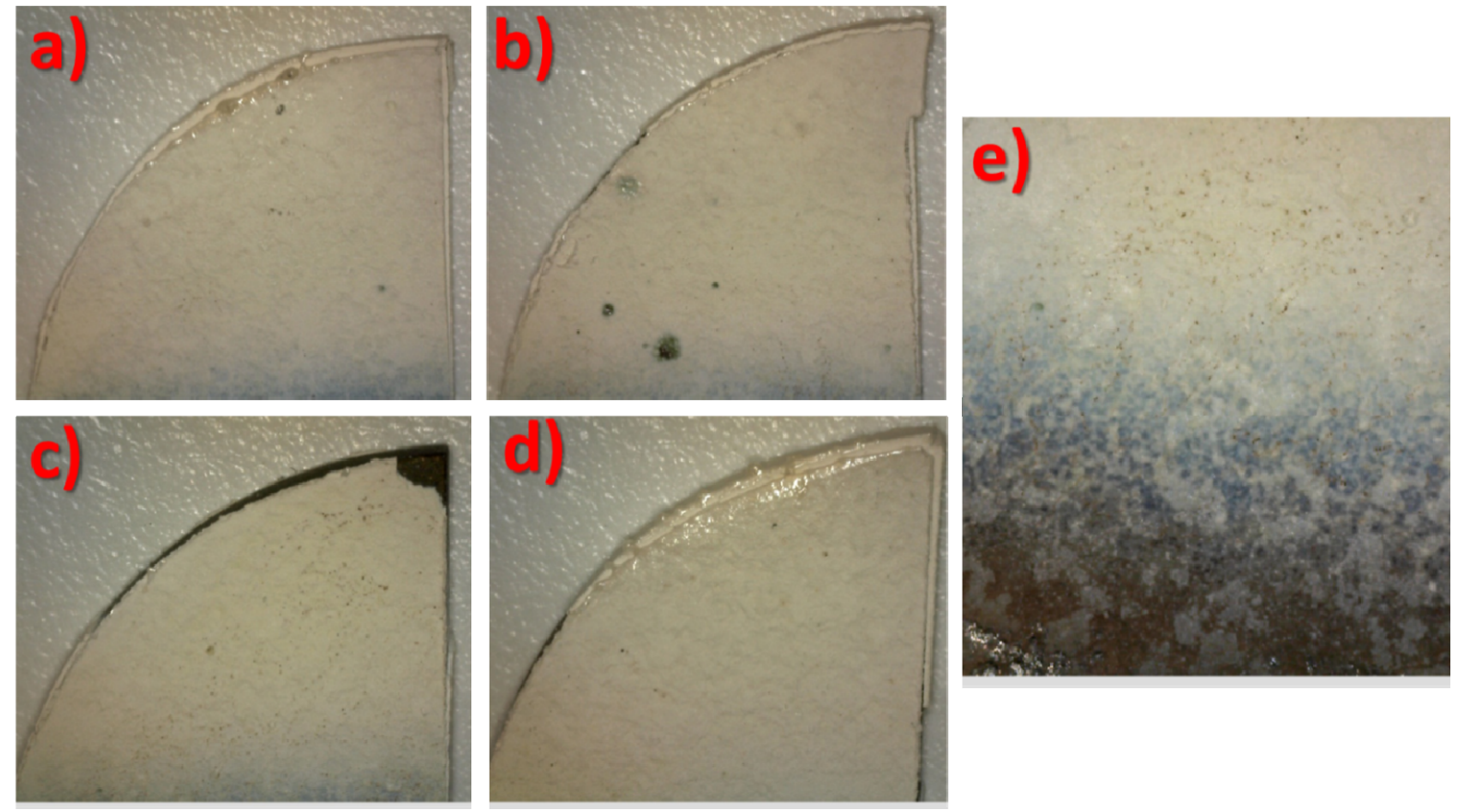

$\mathrm{TaC}$ is the primary reactant in this reaction and this is why the amount of $\mathrm{TaC}$ in the oxidized samples is significantly lower than in the bulk SPS compacts. Other tantalum oxides include $\mathrm{TaO}_{2}$ and $\mathrm{TaO}_{3}$, however these are minor phases and it can be seen that their presence in the oxidized structures is not significantly different than it was in bulk SPS compacts. The presence of the $\mathrm{Ta}_{4} \mathrm{C}_{3}$ phase of tantalum carbide is also not significantly different from the SPS compacts.

The oxidized samples have several phases that do not appear in the SPS compacts, of which most could not be identified. Tantalum metal is found in the TaC sample indicating that some $\mathrm{TaC}$ becomes dissociated in to $\mathrm{Ta}$ and $\mathrm{C}$ when exposed to the high temperature. Interestingly, Ta metal is seen only in the pure $\mathrm{TaC}$ sample and not in the TaC-GNP composites. It is possible that carbon from GNPs react with any Ta metal which forms a tantalum carbide phase - perhaps lower carbon phases like $\mathrm{Ta}_{4} \mathrm{C}_{3}$. 


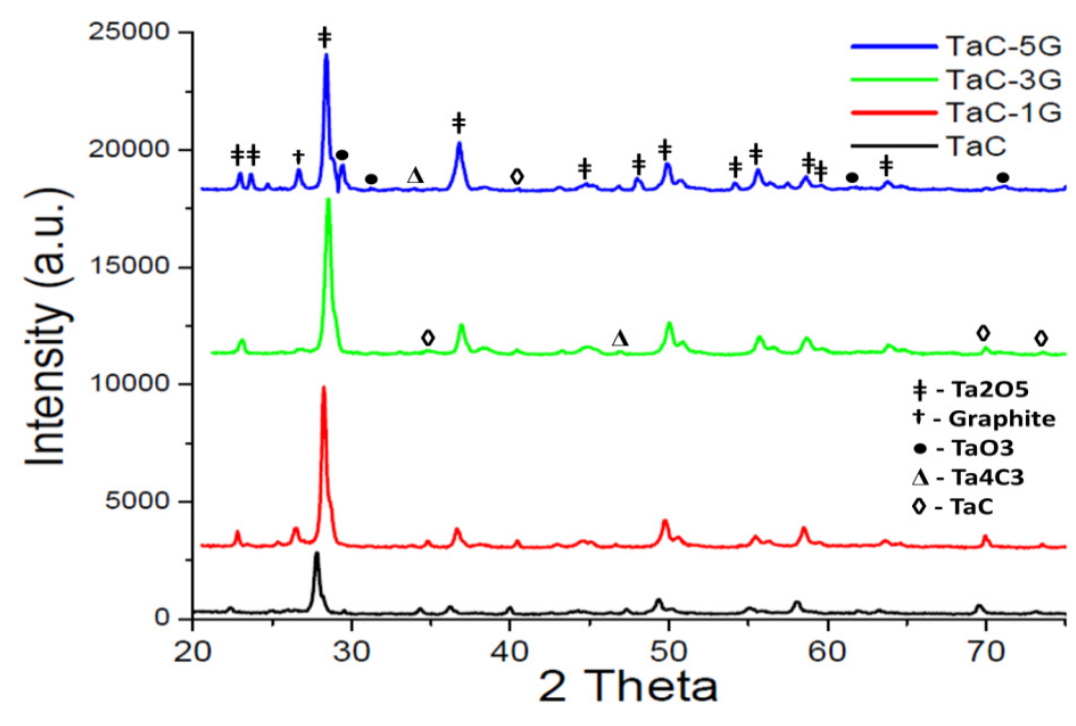

It can be seen in Fig. 4.45e that a blue oxide is observed in some of the oxides. This oxide is seen mostly at the interface between oxidized and unoxidized regions indicating that the tungsten fixture may be the source. Many unknown peaks were observed and this is a consequence of the extreme and rapid conditions experienced along

\begin{tabular}{|c|c|c|c|c|c|c|c|c|}
\hline \multirow[t]{2}{*}{ Oxidation Samples } & \multicolumn{7}{|c|}{ Composition (\%) } & \multirow[b]{2}{*}{ Total } \\
\hline & $\mathrm{TaC}$ & $\mathrm{Ta}_{4} \mathrm{C}_{3} \quad \mathrm{TaO}_{2}$ & $\mathrm{TaO}_{3}$ & $\mathrm{Ta}_{2} \mathrm{O}_{5}$ & $\mathrm{Ta}$ & Graphite & Unknown & \\
\hline $\mathrm{TaC}$ & 6.80 & $2.18 \quad 0.00$ & 1.53 & 77.70 & 5.59 & 3.03 & 3.17 & 100.00 \\
\hline TaC-1G & 5.93 & $0.39 \quad 0.00$ & 1.25 & 81.18 & 0.00 & 6.43 & 4.82 & 100.00 \\
\hline TaC-3G & 4.88 & $0.83 \quad 0.00$ & 0.91 & 85.56 & 0.00 & 1.75 & 6.07 & 100.00 \\
\hline TaC-5G & 0.65 & $0.36 \quad 0.00$ & 9.04 & 76.76 & 0.00 & 4.28 & 8.91 & 100.00 \\
\hline \multirow[t]{2}{*}{ SPS Samples } & \multicolumn{6}{|c|}{ Composition (\%) } & & \\
\hline & $\mathrm{TaC}$ & $\mathrm{Ta}_{4} \mathrm{C}_{3}$ & $\mathrm{TaO}_{2}$ & $\mathrm{TaO}_{3}$ & & $\mathrm{Ta}_{2} \mathrm{O}_{5}$ & & Total \\
\hline TaC & 95.18 & 0.92 & 0.98 & 2.9 & & 0 & & 100.00 \\
\hline TaC-1G & 87.46 & 1.05 & 0 & 5.8 & & 5.67 & & 99.99 \\
\hline TaC-3G & 89.89 & 1 & 1.86 & & 6 & 1.65 & & 100.00 \\
\hline TaC-5G & 90.91 & 2.86 & 1.91 & 4.3 & & 0 & & 100.01 \\
\hline
\end{tabular}


with the complexity of the experimental setup. It is possible that elements from both the fixture as well as the plasma gun may have become embedded in the oxidized samples. Small amounts of the copper anode in the plasma gun may have shot out of the plasma gun as ca $\mathrm{n}$ occur when the plasma gun interior becomes excessively heated. Reactions involving GNP may lead to unexpected and rare phases and may be the reason why the samples with highest GNP concentrations have the highest amount of unknown phases.

\subsection{Oxidation Mechanisms and Microstructural evolution}

Various analyses are done on the oxidized samples in order to quantify their oxidation behavior and specifically the effect of GNP. Our primary mode of quantifying which materials performed the best when exposed to the high temperature plasma flow is to examine the thickness of the resulting formed oxide layers. A thicker oxide layer indicates that the sample has been oxidized to a higher extent than a sample with a thinner oxide layer. Fig. 4.47 provides the thickness of the oxide layers for the $\mathrm{TaC}$ and TaC-GNP composites samples. The thickness of the backside oxide is provided for comparison; however, the rest of the analysis focuses on the front oxide as this is where the most extreme conditions are experienced. The slight differences in the backside oxide behavior will be briefly revisited towards the end of the section.

The oxide thickness of the $\mathrm{TaC}$ sample is by far the highest of any sample. The TaC-GNP samples show decreases in oxide thickness ranging from $50 \%$ to $60 \%$. The TaC-GNP samples show improved oxidation behavior regardless of composition; this 


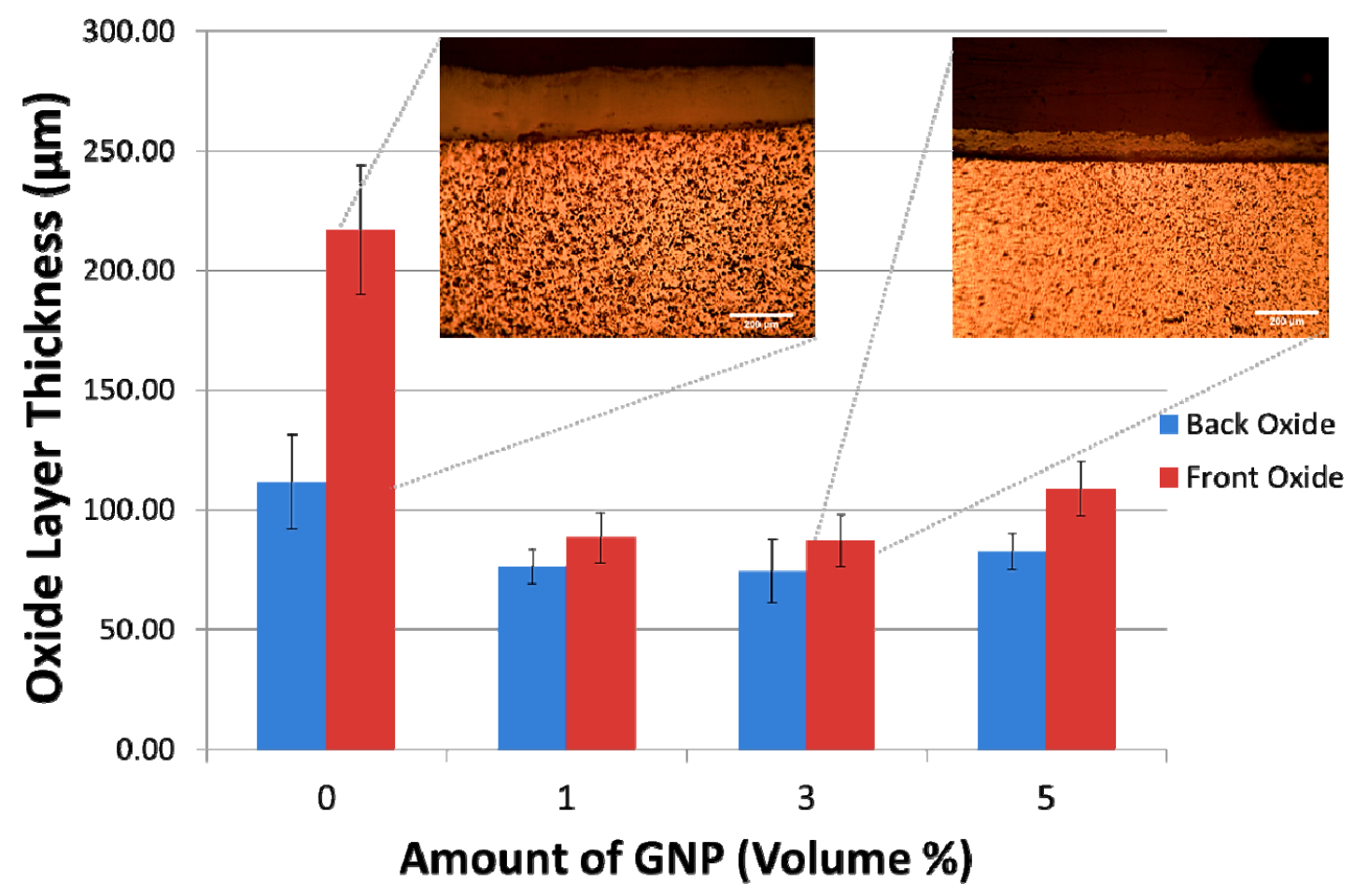

result is significant as it demonstrates that the addition of GNP is not detrimental to the oxidation resistance of $\mathrm{TaC}-$ instead it enhances the oxidation resistance. The improvement in oxidation behavior will be discussed in terms of the following GNP mechanisms and effects: enhanced thermal conductivity, TaC-GNP microstructure, and GNP induced chemical reactions.

\subsubsection{TaC-GNP Thermal Conductivity}

It is well established that GNP is a high thermally conductive phases; graphene sheets have been measured to have a thermal conductivity of $5.1 \times 10^{3} \mathrm{~W} / \mathrm{Mk}$ [110]. The thermal gradient of the samples throughout the experiment were calculated by measuring the backside temperature and assuming that the time required for the front surface of the sample to approach the plasma flow temperature is insignificant. A high thermal gradient 


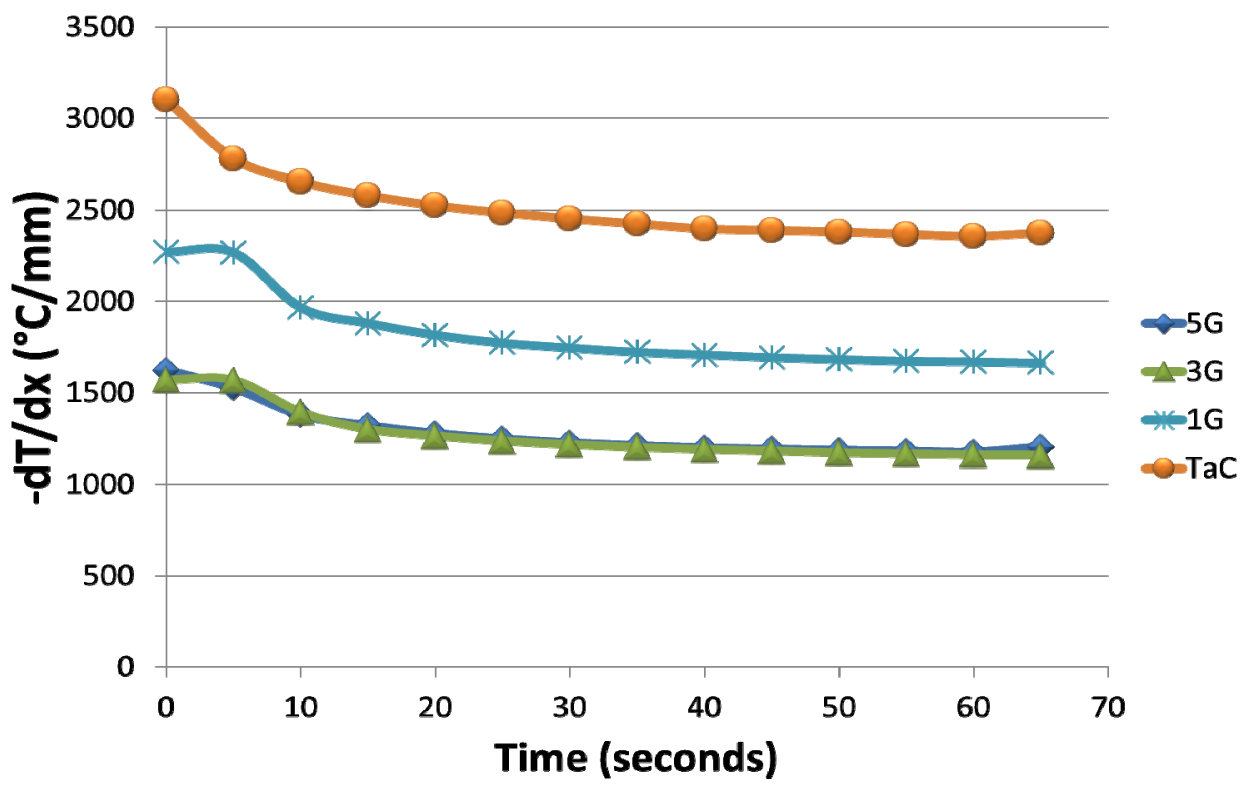

signifies a low thermal conductivity and hence the plots of thermal gradient in Fig. 4.48 give us qualitative information regarding the thermal conductivities of the materials. The TaC-GNP samples have higher thermal conductivity as the GNP enhances the transport of heat throughout the sample as illustrated in Fig. 4.49. The high thermal conductivity of graphene enables the GNP to dissipate heat rapidly during plasma flow exposure.

High thermal conductivity is a desirable property in thermal barrier materials as a highly conductive material will carry heat away from the high flux region and allow heat to be dissipated throughout the sample and radiated in the surroundings. Transporting heat away from the high heat flux region lowers the thermal energy in that region thus decreasing the available energy for formation of oxide phases. Lower surface temperatures at the front surface of the TaC-GNP samples are therefore believed to contribute to the decreased oxide thickness and improved oxidation behavior. 


\section{$\underline{T a C}$}

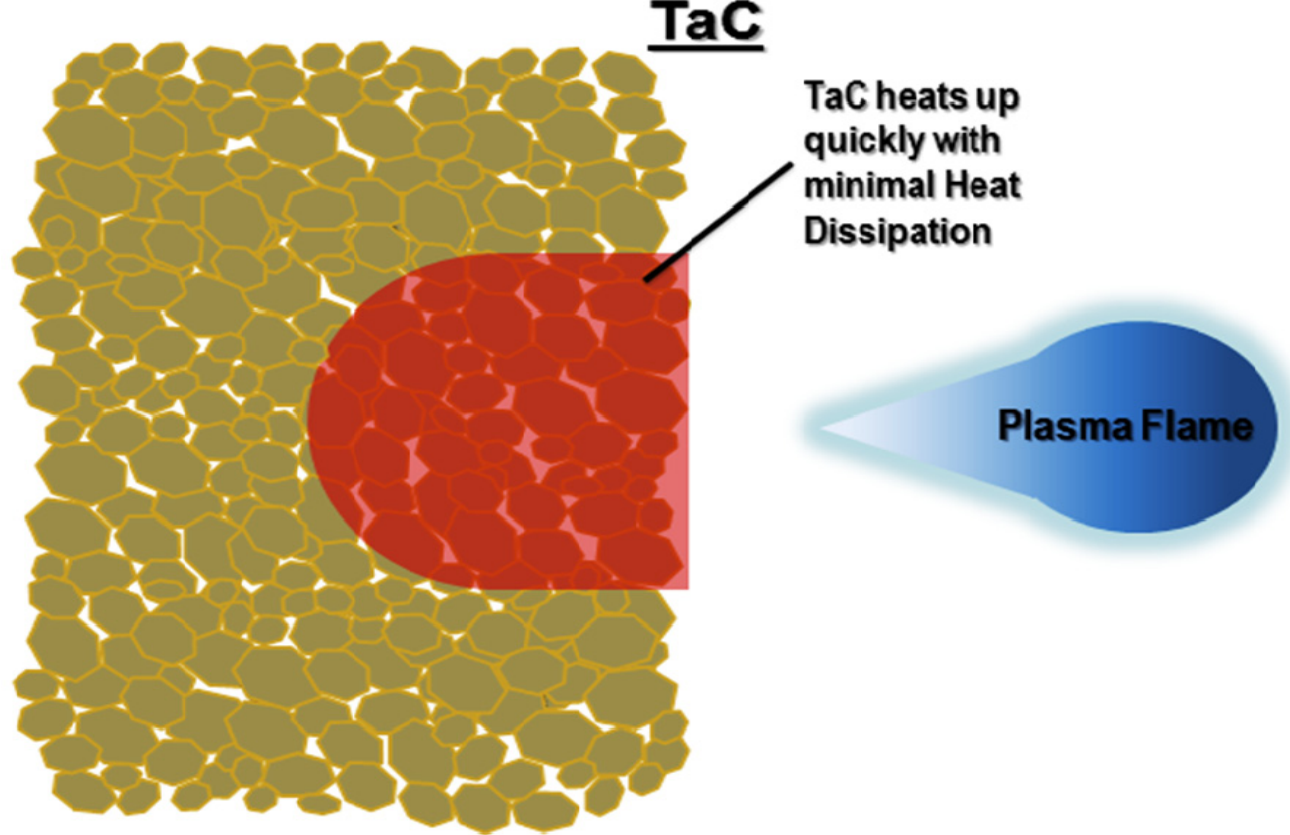

\section{TaC-GNP}

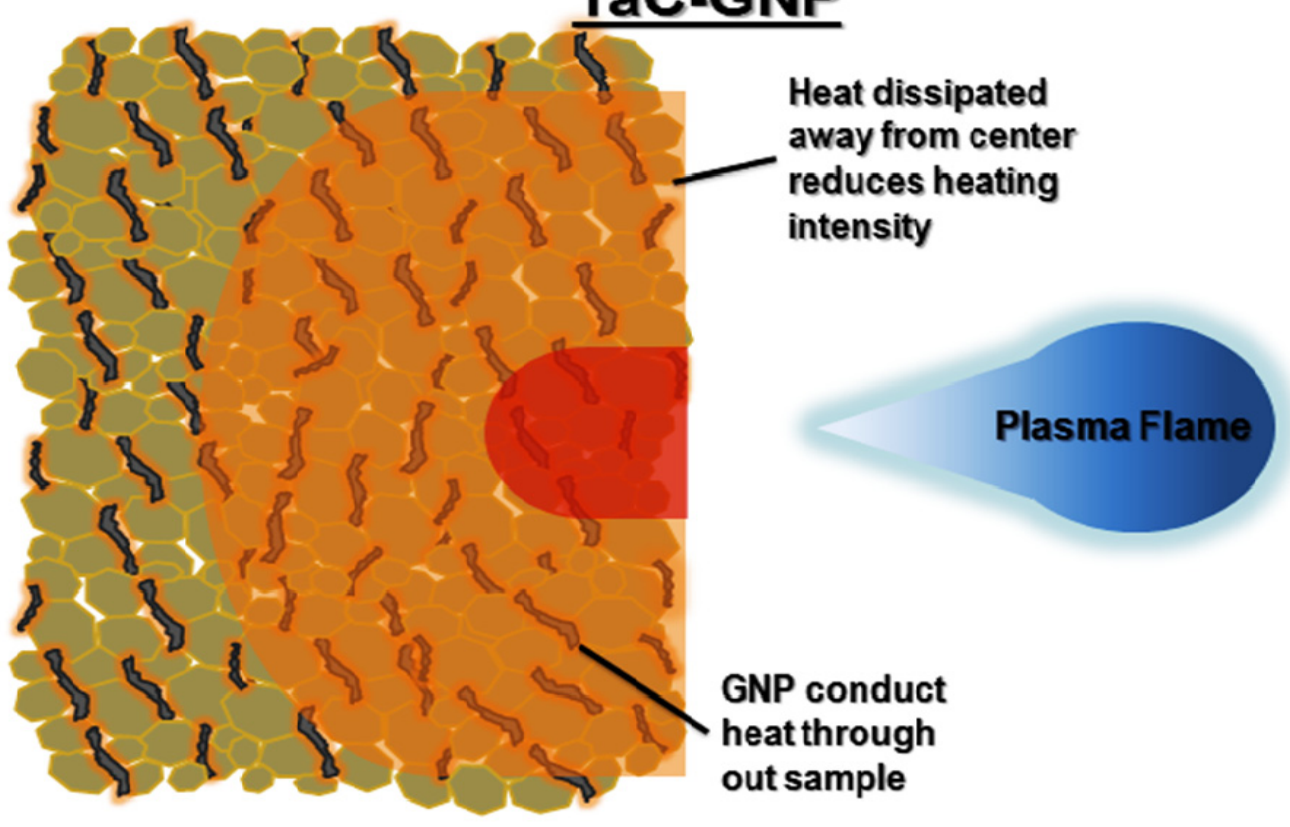




\subsubsection{TaC-GNP Microstructural Mechanisms}

The TaC-GNP composites have microstructural features that significantly impacted the mechanical properties as discussed earlier; some of these same properties have an impact on the oxidation behavior as well. To gain this perspective, we first draw our attention to the oxidized $\mathrm{TaC}$ structure. Fig. $4.50 \mathrm{c}$ and $\mathrm{d}$ show the top surface of the TaC sample after exposure to the plasma flow, the structure is vastly different from the unoxidized top surfaces which are shown in Fig. $4.50 \mathrm{a}$ and b. The surface is obviously heavy oxidized - some oxides have a glassy morphology indicating clear signs of
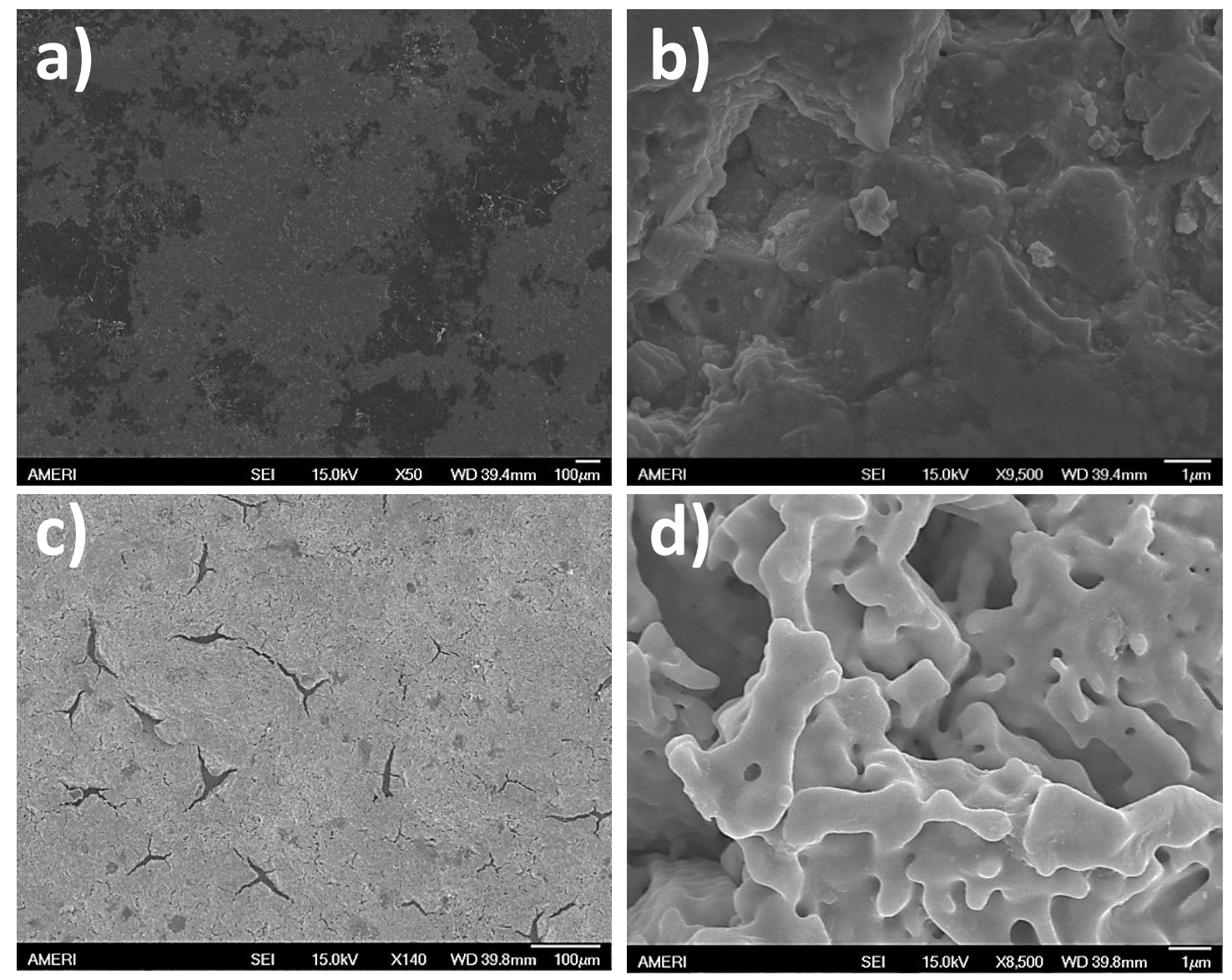
melting. One of the most striking features of the $\mathrm{TaC}$ oxidized structure are the numerous chasms spread out throughout the surface. A closer look at these chasms, Fig. 4.51, reveals that the chasms appear to have formed as a result of some bursting phenomena this bursting is caused by an accumulation of $\mathrm{CO}_{2}$ gases during the primary oxidation reaction (Eqn. 4.2). The high magnification image inside one of these bursts, Fig. 4.51b, reveals porosities scattered uniformly throughout the surface. These porosities are caused by $\mathrm{CO}_{2}$ gases escaping from deep inside the structure. These $\mathrm{CO}_{2}$ gases then accumulate in localized voids in the $\mathrm{TaC}$ structure leading to high pressurization which ultimately causes the gases to burst out of the structure and onto the surroundings. The burst regions then provide large pathways for oxygen to infiltrate and oxidize the sample.

Upon examining the structure of the top surfaces of the TaC-GNP composites, Fig. 4.52, it is seen that the nature of the bursts is vastly different from those in the $\mathrm{TaC}$ structure. Large intense bursts are not seen, instead cracking is observed throughout the sample with signs of bursting on the large cracks. High magnification images of the
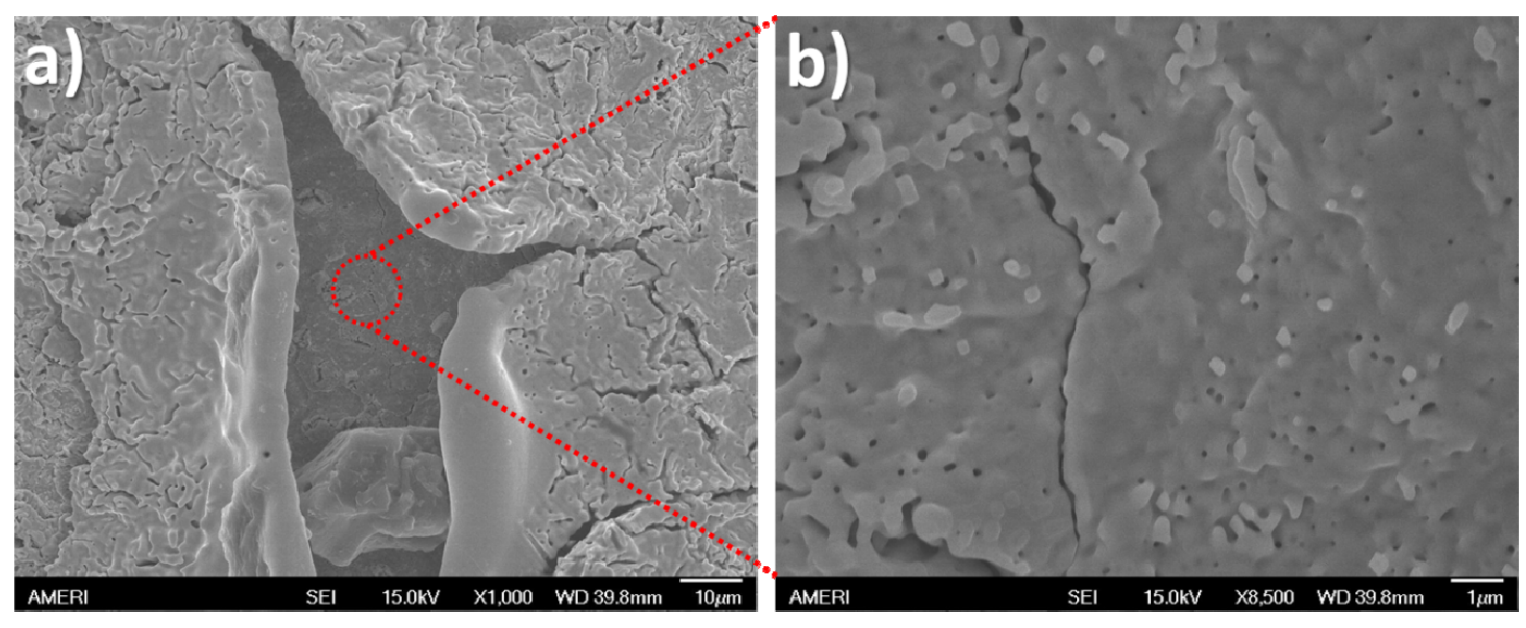
"burst" regions, Fig. 4.52c and d, show that the top surface is peeling away outwards, however it has not burst and left the underlying area exposed as in the $\mathrm{TaC}$ sample. The intensity of the bursts depends on several factors, one of which is the structural integrity of the oxide resisting the high pressure.

The TaC-GNP samples have a higher toughness than the TaC sample as discussed before thus decreasing bursting. The improved structural integrity of the TaC-GNP oxides was explored by measuring the hardness of polished oxide cross sections. The results are shown in Fig. 4.53 and while the precision is low, it can be seen that the trend
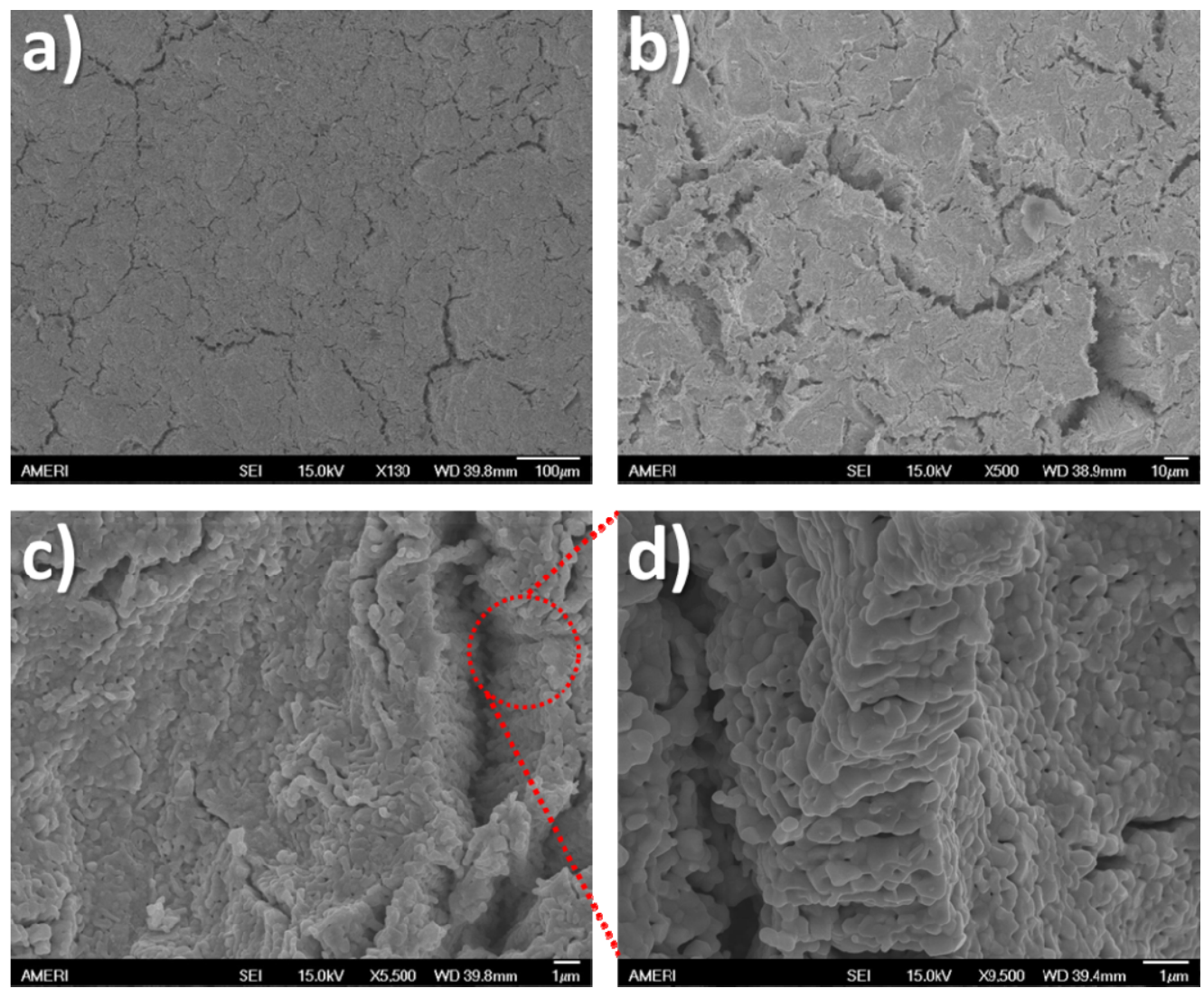
lof the results mirrors the trend seen for fracture toughness. Lower fracture toughness results in more cracking; this compromises the structural integrity of the $\mathrm{TaC}$ oxides which leads to a lower resistance to plastic deformation, i.e. hardness.

Another evident feature in the "burst" regions of the TaC-GNP that is different from the $\mathrm{TaC}$ sample is the structure of the edge of the bursts. Fig. $4.52 \mathrm{~d}$ shows an elongated grain structure indicating TaC-GNP samples are at an early stage of oxidation. In contrast the edges of the burst in the $\mathrm{TaC}$ sample were smooth and glassy; these glassy phases in the $\mathrm{TaC}$ sample imply higher temperatures have been experienced as it indicate $\mathrm{Ta}_{2} \mathrm{O}_{5}$ has not only formed, but it has reached its melting point $\left(1872{ }^{\circ} \mathrm{C}[150]\right)$ and resolidified. The TaC-GNP composite's higher toughness and higher thermal conductivity contributes to the decreased and less intense bursting - less burst regions mean there are less pathways for oxygen to diffuse into the structure.

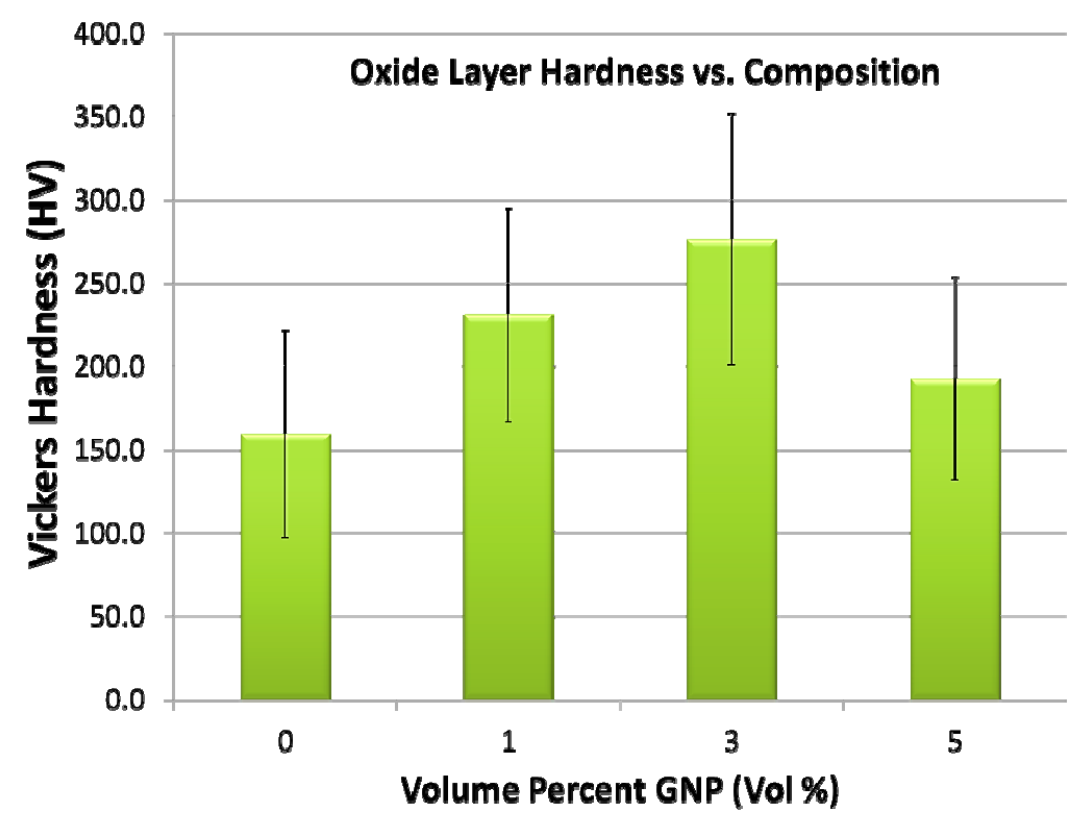




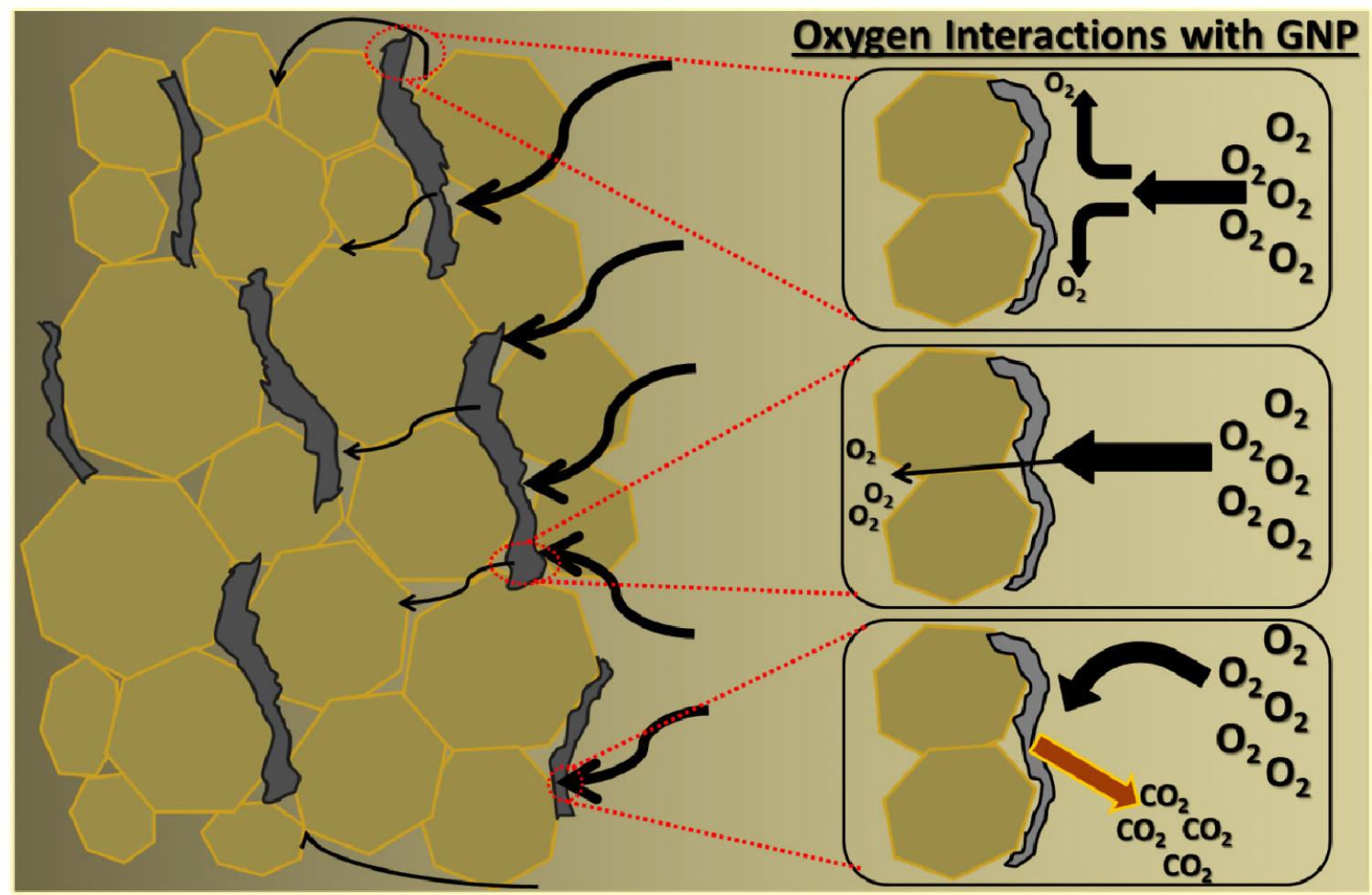

The GNP may also inhibit diffusion of oxygen into the structure in more direct ways. Oxygen diffusion is most likely to occur in between $\mathrm{TaC}$ grains and thus the grain boundaries essentially provide a diffusion path for oxygen molecules. SEM analysis in previous sections revealed that GNP often wrap around and weave through grains in the TaC-GNP samples. As such, it is proposed that GNP may "seal" some of the oxygen diffusion paths by making the diffusion of oxygen through grain boundaries more arduous. The proposed mechanism is illustrated in Fig. 4.54; oxygen molecules encountering a GNP have three plausible options. They may diffuse through the GNP which uses up energy otherwise spent diffusing through the grain boundary. The oxygen may chemically interact with the GNP forming either graphite oxide or gas products such carbon dioxide. The presence of graphite oxide was not observed in the XRD analysis of 
the TaC-GNP oxidized top surfaces. The third option for molecules encountering a GNP would be to find another more favorable diffusion path which would entail the oxygen traveling laterally (i.e. perpendicular to the plasma flow direction) before going deeper into the sample.

\subsubsection{GNP Induced Chemical Reactions}

Another powerful GNP mechanism in improving oxidation behavior is the chemical reaction that occurs when GNP or any other carbon source reaction with tantalum pentoxide at temperatures above $1135^{\circ} \mathrm{C}$. The reaction is shown in Eqn. 4.3 and it can be seen that the product of this reaction lead to the formation of tantalum carbide, thus essentially reversing the oxidation process.

$$
\mathrm{Ta}_{2} \mathrm{O}_{5}+7 \mathrm{C} \rightarrow 2 \mathrm{TaC}+5 \mathrm{CO}(\Delta G=1142.3-.823 \mathrm{~T}),\left(\mathrm{T}>1135^{\circ} \mathrm{C}\right)
$$

This reaction will therefore occur after the formation of $\mathrm{Ta}_{2} \mathrm{O}_{5}$ by the reaction in Eqn. 4.1 provided there is a source of carbon. The GNP show that they can survive the plasma flow conditions to a certain extent and therefore they can provide the source of carbon needed for the reaction in Eqn. 4.3 to take place. It should be emphasized that these reaction does not occur immediately upon exposure of the $\mathrm{TaC}$ based composites to the plasma flow - some oxidation of $\mathrm{TaC}$ into $\mathrm{Ta}_{2} \mathrm{O}_{5}$ must occur. It is likely that by the time the reaction of $\mathrm{Ta}_{2} \mathrm{O}_{5}$ back to $\mathrm{TaC}$ begins to occur, some degree of bursting or cracking has already occurred. These cracked or burst regions provide pathways for the CO products to escape and therefore the $\mathrm{CO}$ should not have a significant adverse effect on the structural integrity or oxidation behavior of the TaC-GNP samples. 
EDS analysis were conducted on the samples to quantify the amounts of carbon, oxygen, and tantalum present in order to gauge the plausibility and extent of the reaction just described. EDS analysis on the cross section of each sample is provided in Fig. 4.55. The TaC-GNP samples show two general features: the amounts of carbon in the oxide layers are greater than the amounts of oxygen, and the amount of tantalum in the oxide layer increases sharply near the interface. The TaC sample has a higher amount of oxygen than carbon throughout the oxide and the amount of tantalum increases modestly as the interface is approached.

The increased amounts of carbon in the TaC-GNP samples are a result of the surviving GNP in the oxide structure. TaC-3G had the most uniformly distributed
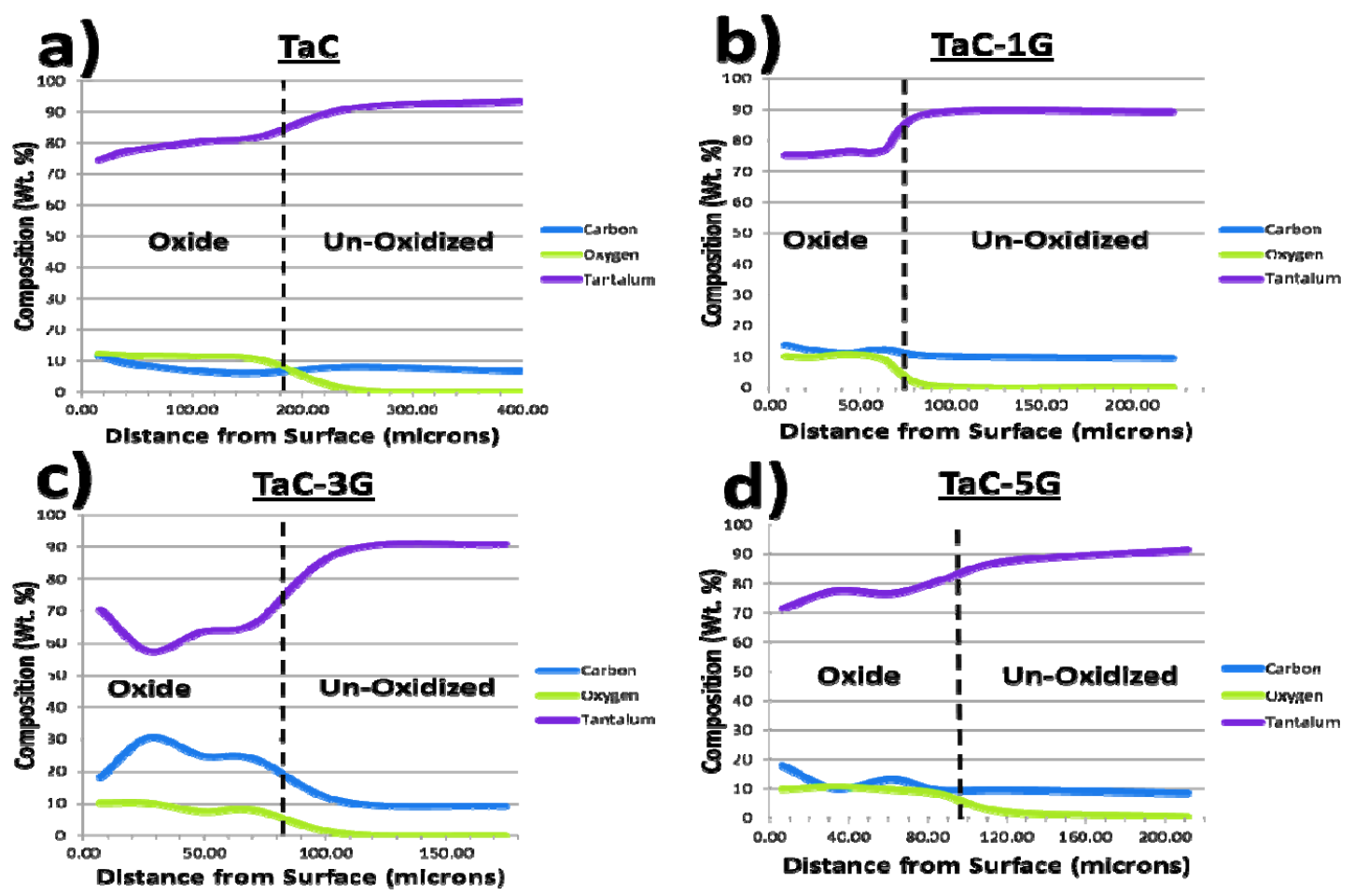
GNPand thus the TaC-3G sample displays the highest amounts of carbon in the oxide structure. The other reason there is more carbon than oxygen in the TaC-GNP samples is that the amount of oxygen in the TaC-GNP samples is lower than in the TaC samples this signifies that there is less $\mathrm{Ta}_{2} \mathrm{O}_{5}$ in the TaC-GNP oxide layers. The $\mathrm{Ta}_{2} \mathrm{O}_{5}$ is reacting with the excess carbon provided by GNP and forming TaC, while the oxygen escapes out as $\mathrm{CO}$. The amount of Ta rises sharply in the TaC-GNP composites because there is more $\mathrm{TaC}$ near the interface, the denser $\mathrm{TaC}$ gives a higher Ta count than $\mathrm{Ta}_{2} \mathrm{O}_{5}$. There is more $\mathrm{TaC}$ near the interface in the TaC-GNP samples because there will be more surviving GNP near the interface which provide the carbon needed to convert $\mathrm{Ta}_{2} \mathrm{O}_{5}$ to $\mathrm{TaC}$. More GNPs are expected to survive deeper in the sample as the $\mathrm{TaC}$ and $/$ or $\mathrm{Ta}_{2} \mathrm{O}_{5}$ will insulate the GNP from the high temperatures.
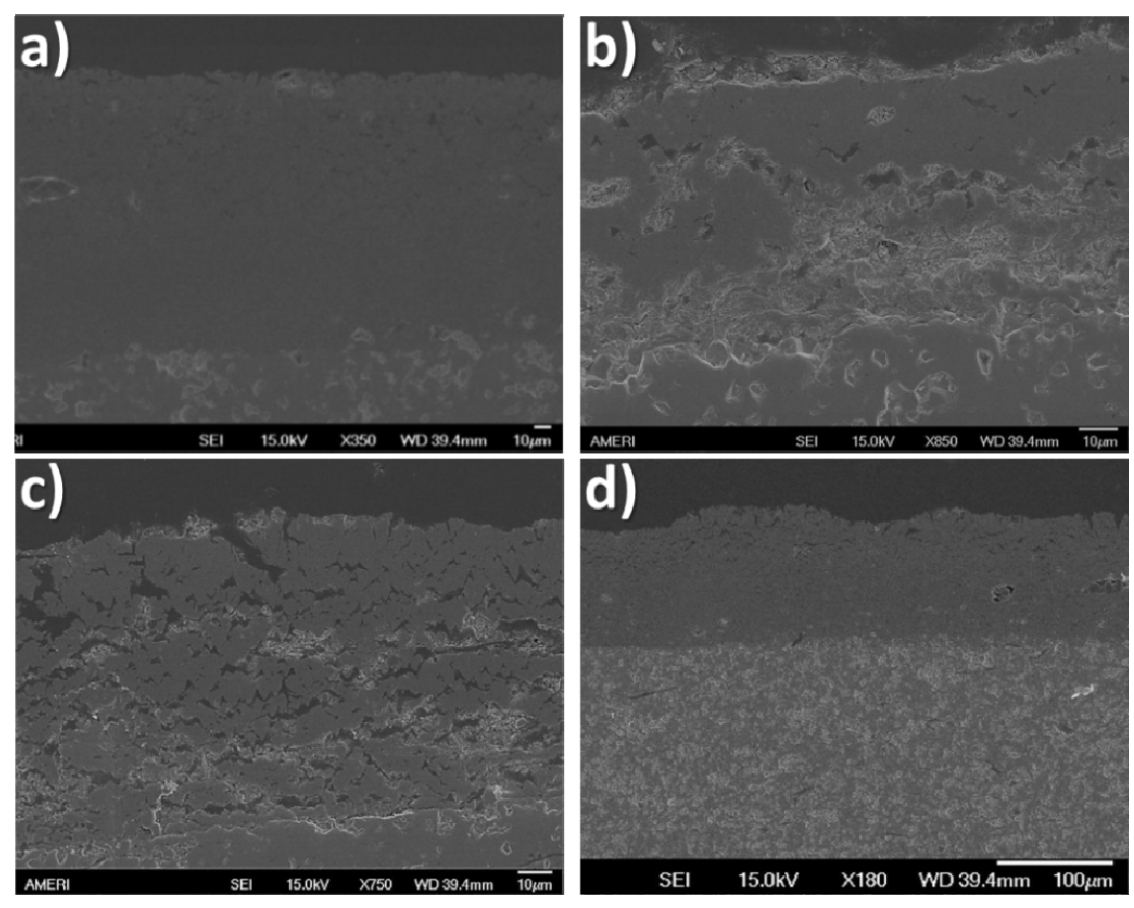

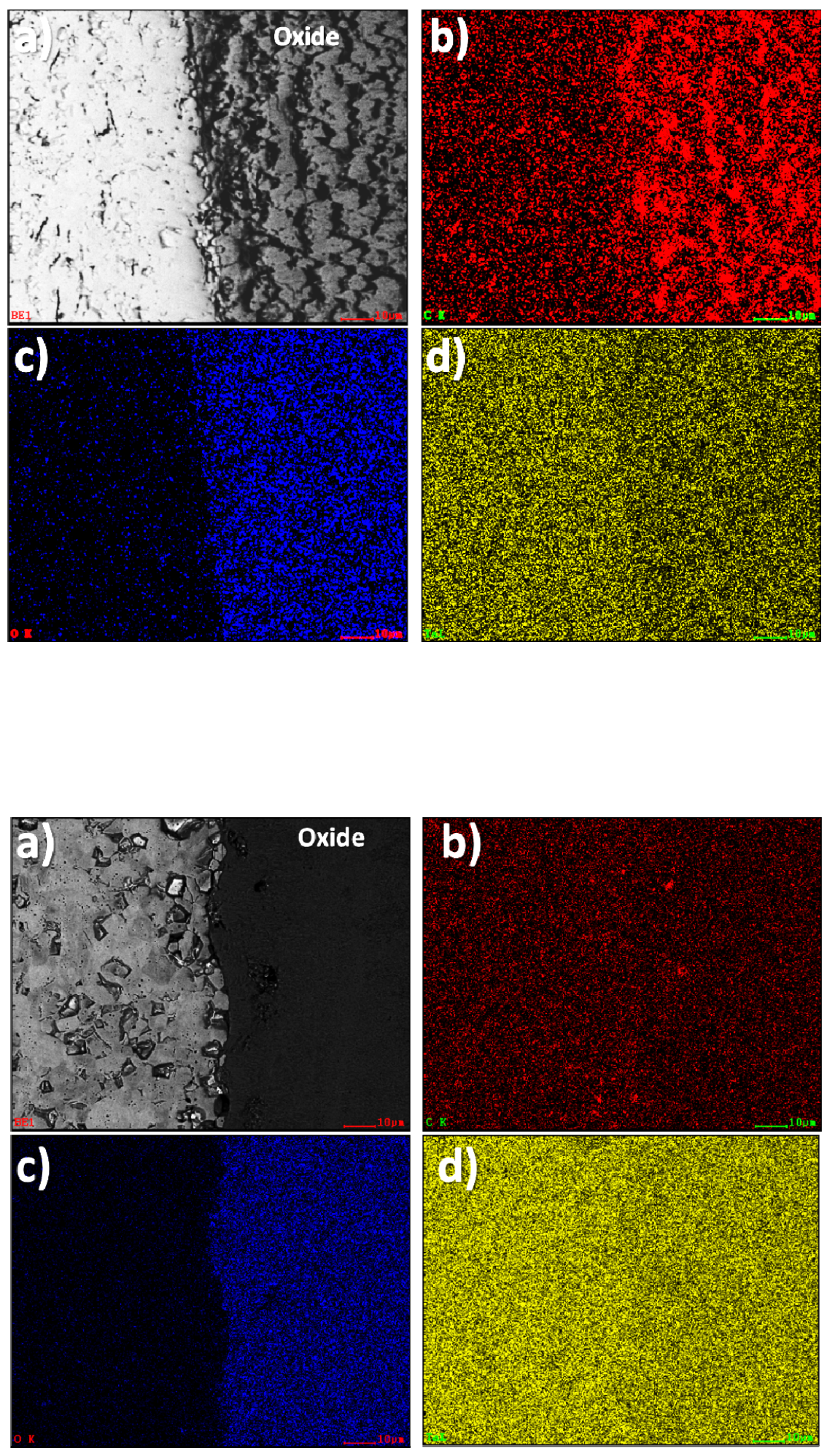

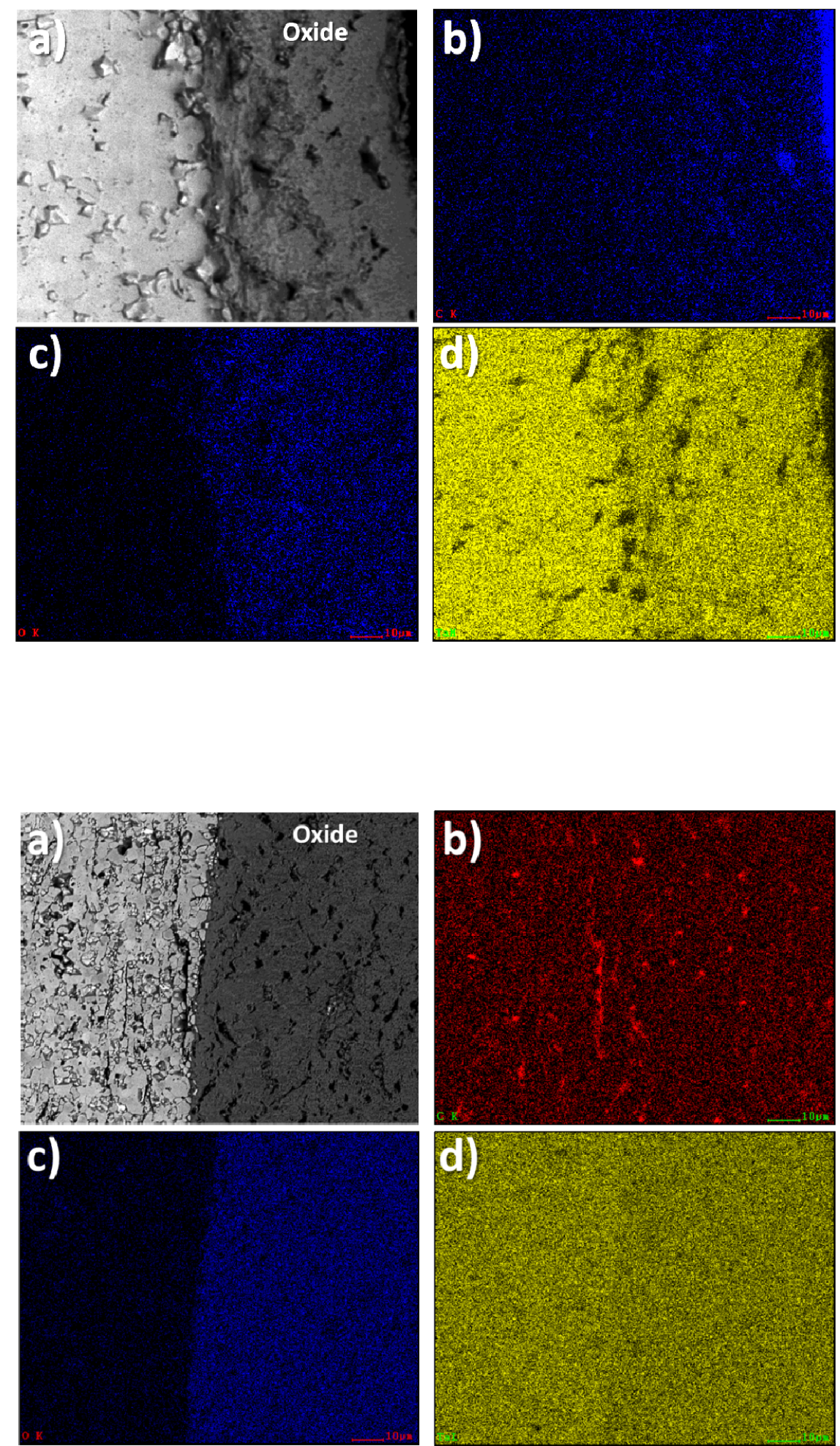
Typical cross sections in which the EDS analyses were performed for each sample are shown in Fig. 4.56. The TaC-GNP samples display a more porous structure as indicated by the darker regions. The TaC-3G sample has the most prevalent and uniform presence of darker regions; an $\mathrm{x}$-ray map of the TaC-3G oxidized and unoxidized regions is provided in Fig. 4.57 in order to verify the composition of the dark regions. Figs. 4.584.60 provides $\mathrm{x}$-rays maps of the remaining samples as reference. The dark regions in the TaC-3G structure are very rich in carbon. The carbon signal is high because these are porous regions and thus the epoxy used to mount the samples has infiltrated the pores. The pores are also high in carbon because these pores are the result of carbon which has been consumed during the oxidation process - this is why no such structures are observed in the pure $\mathrm{TaC}$ sample. The carbon may be consumed either during the conversion of $\mathrm{Ta}_{2} \mathrm{O}_{5}$ to $\mathrm{TaC}$ or directly from solid carbon to $\mathrm{CO}$ and $\mathrm{CO}_{2}$ due to the high temperatures. The porous structures are most prevalent in the TaC-3G sample because the GNP were most uniformly distributed in that sample. The x-ray map reveals the expected results of higher oxygen and lower Ta in the oxide layer, however, neither shows any preferential distribution in the structure.

The oxidation behavior of the backside of the samples is now discussed as the primary factor causing a variation in behavior is the reduced impact of the GNP chemical reactions. The reaction between carbon from GNPs and $\mathrm{Ta}_{2} \mathrm{O}_{5}$ has a reduced impact because in the backside there is less time when this reaction is thermodynamically favorable. The temperature at the backside of the sample will be significantly lower than in the front surface. The formation of the oxide layer is therefore delayed or at least 
slower compared to oxide formation rate in the front surface. The reaction of $\mathrm{Ta}_{2} \mathrm{O}_{5}$ to $\mathrm{TaC}$ requires temperatures above $1135{ }^{\circ} \mathrm{C}$ with even higher temperatures providing a higher driving force. The reaction converting $\mathrm{Ta}_{2} \mathrm{O}_{5}$ to $\mathrm{TaC}$ is most effective at extreme conditions when there is a high rate of $\mathrm{Ta}_{2} \mathrm{O}_{5}$ formation and high temperatures to accelerate the $\mathrm{Ta}_{2} \mathrm{O}_{5}$ conversion to $\mathrm{TaC}$, granted there is a sufficient source of carbon. The enhanced thermal conductivity which decreases localized temperature, and the GNPs which seal off grain boundaries and hinder diffusion of oxygen into the structure are likely the predominant mechanisms that result in decreased back-side oxide thickness in the TaC-GNP samples.

\subsubsection{Oxidation behavior variations in TaC-GNP composites}

The variations in oxidation behavior, albeit minimal, are due to the differences in GNP distribution across the different compositions. To better gauge the variations in oxidation behavior, the surface recession experienced by each sample is shown in Fig. 4.61. In accordance with the previous results, the surface recession in all the TaC-GNP samples is greatly reduced when compared with the $\mathrm{TaC}$ sample. The surface recession in the $\mathrm{TaC}-3 \mathrm{G}$ sample however is slightly higher than in the $\mathrm{TaC}-1 \mathrm{G}$ and $\mathrm{TaC}-3 \mathrm{G}$ samples. The TaC-3G sample has the best distribution of GNP and therefore the heat is transferred most efficiently in this sample. The TaC-3G sample was shown to have a superior thermal conductivity as compared to the $\mathrm{TaC}-1 \mathrm{G}$ sample and therefore higher temperatures were experienced deeper in the $\mathrm{TaC}-3 \mathrm{G}$ sample than in the $\mathrm{TaC}-1 \mathrm{G}$ sample.

This higher temperature experienced leads to more of the surface becoming oxidized and thus the unoxidized region recedes further. The TaC-5G sample has a similar thermal 
conductivity to TaC-3G however the uniformity of the GNPs is not as high as in the TaC3G sample. There may be large swaths of $\mathrm{TaC}$ without any GNP and thus the penetration of heat will be lower in $\mathrm{TaC}-5 \mathrm{G}$ than in $\mathrm{TaC}-3 \mathrm{G}$.

Despite the higher surface recession in $\mathrm{TaC}-3 \mathrm{G}$, the oxide thickness is the lowest of all the samples and this is because the GNP induced conversion of $\mathrm{Ta}_{2} \mathrm{O}_{5}$ to $\mathrm{TaC}$ is most efficient. The TaC-1G sample has less GNP available than the TaC-3G sample. The TaC-5G sample has more GNP than TaC-3G however the distribution is not uniform and pores were often seen at interface between TaC and GNP grains. Also, GNP in TaC-5G was often agglomerated into large thick chunks - higher relative surface area in TaC-3G will provide more carbon for the reduction of $\mathrm{Ta}_{2} \mathrm{O}_{5}$ to $\mathrm{TaC}$. The cross section micrographs in Fig. 4.56confirm the GNP consumption was higher in TaC-3G than in the other samples as the presence of dark regions (porous regions w/ remnants of GNP), is greater and more uniform than in any other sample.

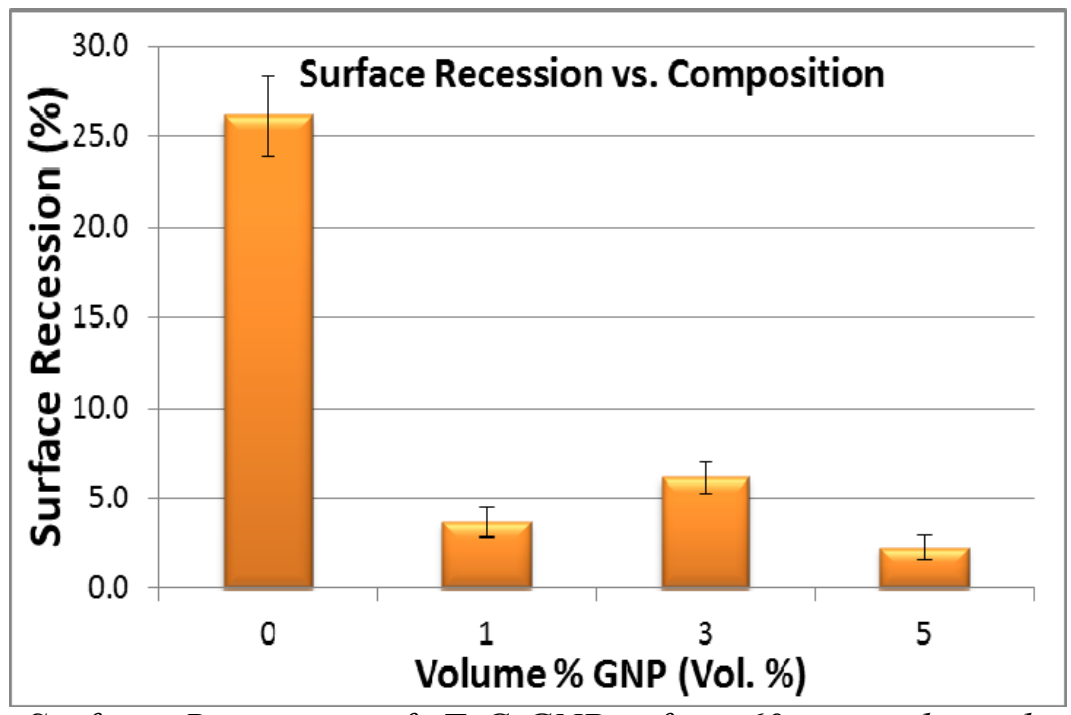

Fig. 4.61: Surface Recession of TaC-GNP after 60 second oxidation across compositions. Percentage is relative to original sample thickness 
The various GNP mechanisms proposed are intertwined making comparisons between different GNP compositions difficult. It is not immediately obvious for example why the oxide thickness in TaC-1G and TaC-3G was nearly identical. There may be an optimum thermal conductivity for example where heat is transferred efficiently away from the sample without bringing excess heat deeper into the sample. Overall, the combination of various GNP mechanisms leads to greatly improved oxidation behavior over the unreinforced $\mathrm{TaC}$ sample.

\subsection{GNP Structure}

Throughout this research, an emphasis has been placed on the structure of GNP and its interactions with the $\mathrm{TaC}$ matrix - the oxide structure is no exception. Unlike TaC, GNP is not highly resistive to corrosion and oxidation and thus the survival of the GNP in the plasma flow was not certain. The extent of GNP survival is important in assessing how long GNP mechanisms can be effective during oxidation. Survival of GNP is especially critical to the localized reducing environments believed to reduce $\mathrm{Ta}_{2} \mathrm{O}_{5}$ into TaC. If GNP don't survive long enough, then the formation of even localized reducing environments is less plausible.

Raman spectroscopy is used to access the survival and structure of GNPs in the oxidized TaC-GNP structure. The Raman spectra of are presented in Fig. 4.62 and in Table 4.9 a summary of the relevant peak intensity ratios is provided - results for the SPS bulk samples are repeated for comparison clarity. In all samples, the drastic reduction in a 


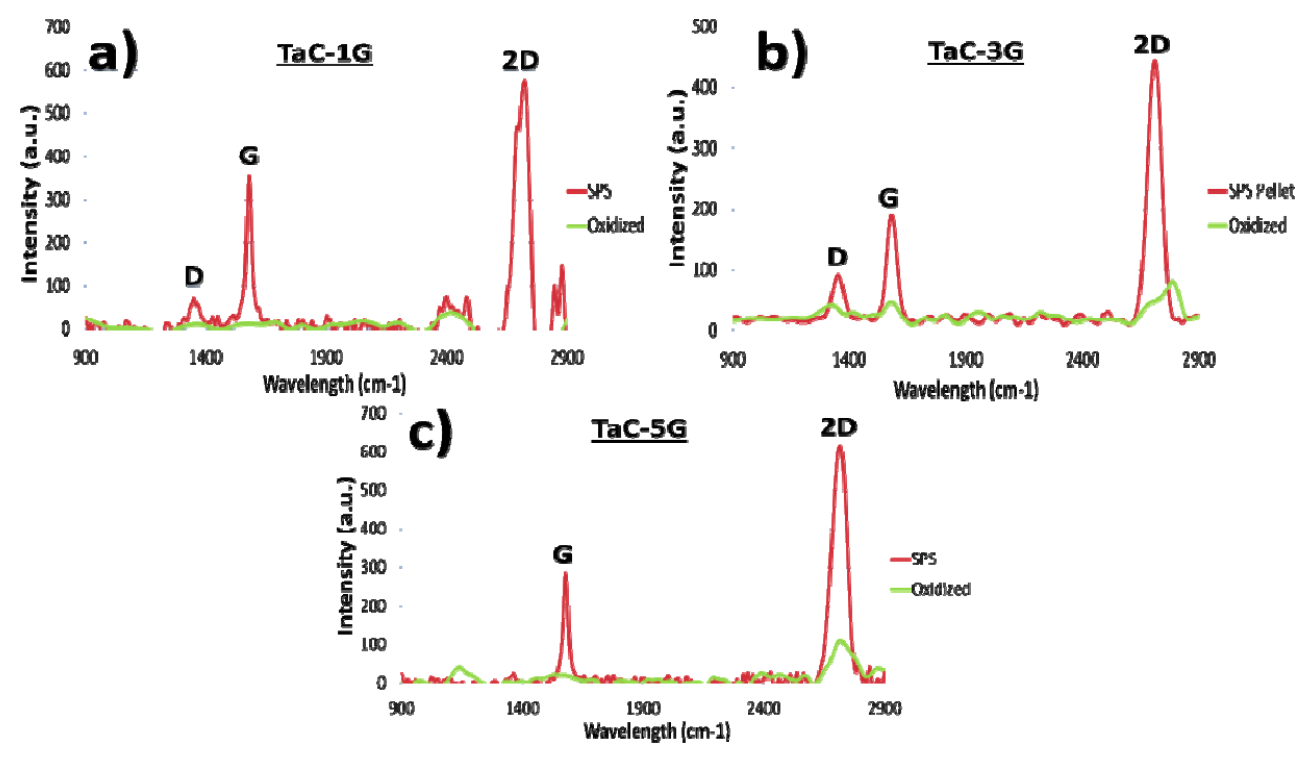

graphene or graphite structure signature is evident. The exposure to the high temperature plasma has left little or no traces of GNP in the TaC-GNP oxides. The oxidized TaC-1G structure reveals no facets of a carbon structure. The only peaks in the $\mathrm{TaC}-1 \mathrm{G}$ samples occurs at $\sim 2400 \mathrm{~cm}^{-1}$ and this is a noise peak which is also present in a similar fashion in the bulk SPS structure of $\mathrm{TaC}-1 \mathrm{G}$.

\begin{tabular}{|cccccc}
\hline Sample & D Peak & G Peak & 2D Peak & $\mathbf{I}_{\mathbf{D}} / \mathbf{I}_{\mathbf{G}}$ & $\mathbf{I}_{\mathbf{2 D}} / \mathbf{I}_{\mathbf{G}}$ \\
$\begin{array}{c}\mathbf{1 G} \\
\text { Oxidized }\end{array}$ & 1365.76 & $\mathrm{n} / \mathrm{a}$ & $\mathrm{n} / \mathrm{a}$ & $\mathrm{n} / \mathrm{a}$ & $\mathrm{n} / \mathrm{a}$ \\
$\begin{array}{c}\text { 3G } \\
\text { Oxidized }\end{array}$ & 1325.66 & 1579.56 & 2786.28 & 0.817 & 2.306 \\
$\begin{array}{c}5 \mathrm{5G} \\
\text { Oxidized }\end{array}$ & $\mathrm{n} / \mathrm{a}$ & $\mathrm{n} / \mathrm{a}$ & 2716.47 & $\mathrm{n} / \mathrm{a}$ & $\mathrm{n} / \mathrm{a}$ \\
\hline 16 SPS & 1352.02 & 1579.56 & 2723.35 & 0.193 & 1.613 \\
\hline 3G SPS & 1352.02 & 1580.73 & 2709.57 & 0.416 & 2.505 \\
\hline 5G SPS & 1364.51 & 1579.56 & 2716.47 & 0.080 & 2.169 \\
\hline
\end{tabular}


The oxidized $\mathrm{TaC}-3 \mathrm{G}$ and $\mathrm{TaC}-5 \mathrm{G}$ samples display minor peaks associated with carbon structures. The TaC-3G sample displays a small G Peak, along with shifted D and 2D peaks. The peaks allow us to compute the $\mathrm{I}_{\mathrm{D}} / \mathrm{I}_{\mathrm{G}}$ and $\mathrm{I}_{2 \mathrm{D}} / \mathrm{I}_{\mathrm{G}}$ ratios and compare them with those of the bulk SPS structure. The oxidized sample display a higher $I_{D} / I_{G}$ ratio which reflects the significant amount of damaged incurred to the GNP structure during plasma flow exposure. Similarly, the $\mathrm{I}_{2 \mathrm{D}} / \mathrm{IG}$ ratio is lower in the oxidized sample indicating the loss of a graphene-like structure. The TaC-5G sample only displays a small peak at $2700 \mathrm{~cm}^{-1}$; this $2 \mathrm{D}$ peak indicates that a few remnants of GNP and graphene sheets can be found in the oxidized structure. The peaks observed in both TaC-3G and TaC-5G are wide and this may indicate the carbon left in the TaC-GNP structure is of an amorphous nature. Amorphous carbon will tend to give wider peaks while defined structures such as graphene and CNT have sharper more well defined peaks [88].

The Raman spectra indicate the presence of small amounts of carbon structures, for further clarity on the nature of these structures the oxidized samples are observed under SEM. Various structures resembling agglomerated GNP or graphite are seen in the TaC-GNP samples as shown in Fig. 4.63. The carbon structures in Fig. 4.63a and 4.63b are engulfed in the oxidized $\mathrm{TaC}$ indicating these carbon structures originated from the sample. The carbon structure on the TaC-3G sample shows strong interaction with the oxide, $\mathrm{TaC}$ oxides can be seen on the surface of the carbon structure indicating some degree of wrapping present. The TaC-3G sample displayed large amounts of GNP weaving and wrapping around $\mathrm{TaC}$ grains in the SPS bulk structure. Other distinct carbon structures are seen in the oxides such as the tubular structure seen in Fig. 4.63d; this 

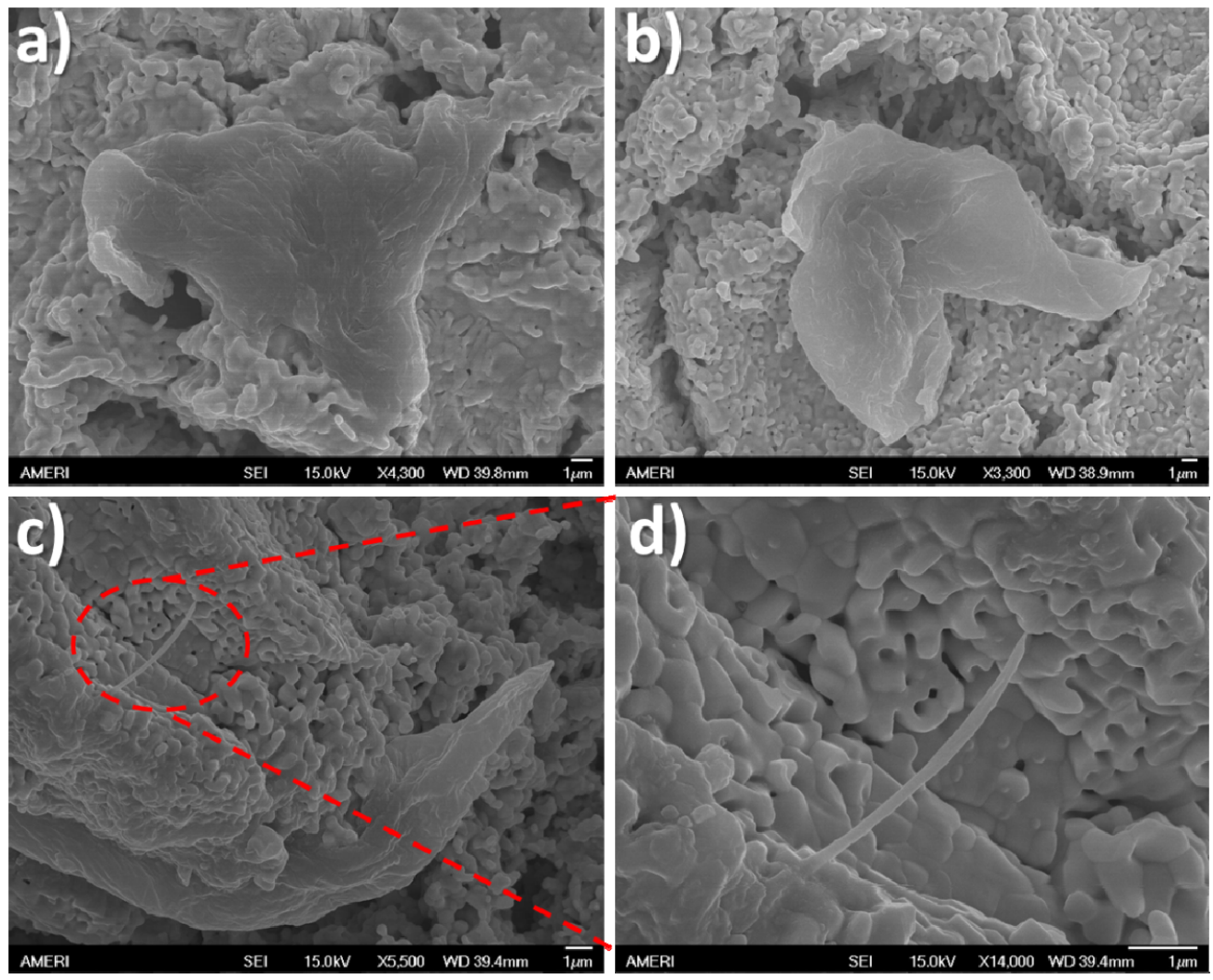

structure resembles a graphene nanoribbon and is indicative of the high level of interaction between GNP and $\mathrm{TaC}$.

Other highly immersed tubular carbon structures can be seen in the top surfaces and cross sections of the TaC-GNP samples. These "bands" of carbon are deeply embedded in the oxides of all the compositions, which may provide a toughening of the oxide similar to the toughening observed in the bulk structures. The structure of the bands varies; Fig. 4.63d and Fig. 4.63b shows smooth, few micron long bands while Fig. 4.64a and Fig. 4.64d show rough bands spanning only a few hundred nanometers. The varying sizes of bands indicate potential for multi-scale toughening of the $\mathrm{TaC}$ based oxides. 
Close inspection of the 'rough' bands reveals that the carbon bands have been wetted by $\mathrm{TaC}$ based oxides. The small size of the oxides on the bands indicates that they may have solidified from a molten state, hence demonstrating the ability of GNP to survive the extreme temperatures needed to melt $\mathrm{TaC}$.

Fig. 4.65 reveals that large GNPs in the TaC-3G and $\mathrm{TaC}-5 \mathrm{G}$ sample have survived the high temperature plasma exposure. This is consistent with the Raman Spectra which indicated some level of graphene/graphite structure present in the TaC-3G and TaC-5G samples. The structures in Fig. 4.65 show distinct features of GNP such as a high aspect ratio and smooth surfaces with occasional crinkles. The presence of large

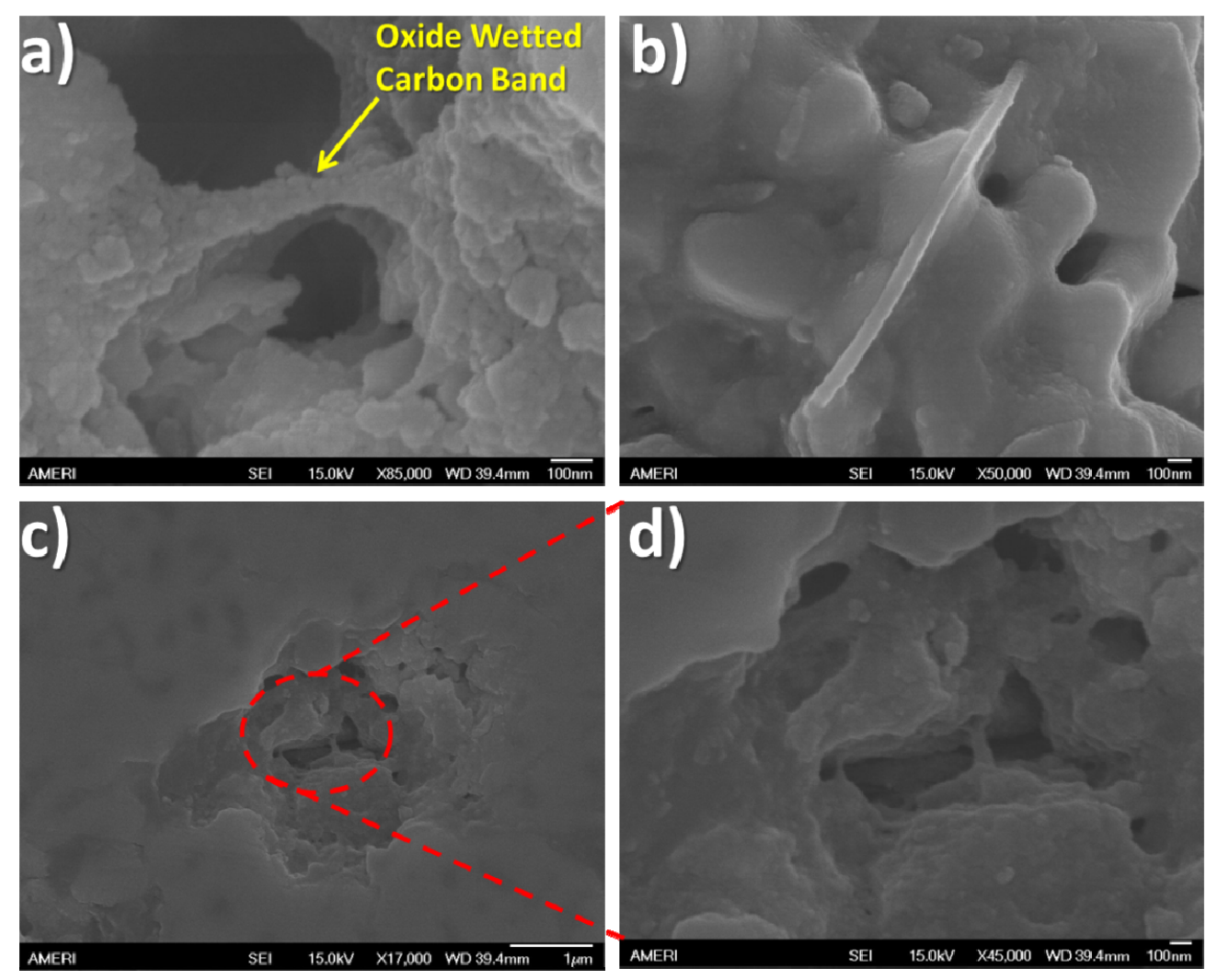


GNPs indicates that GNP may survive even when they are not immersed and protected by

$\mathrm{TaC}$.
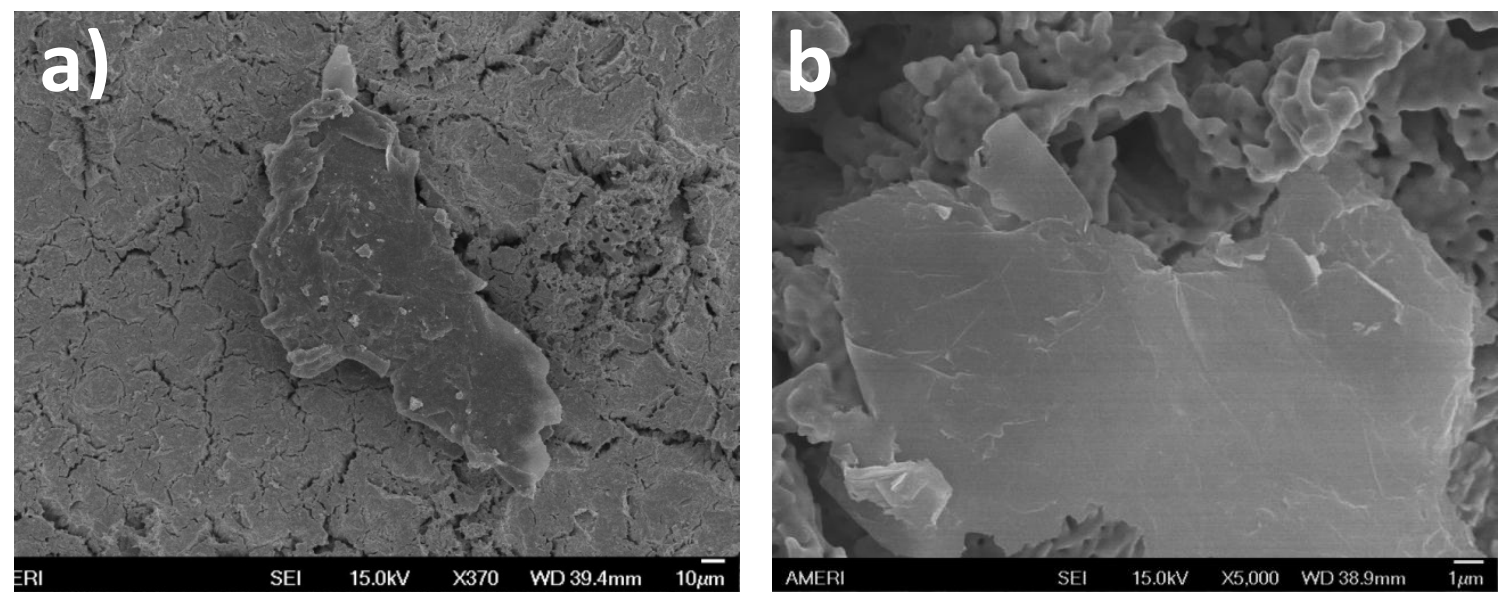


\section{CHAPTER V: CONCLUSIONS}

In this Thesis Graphene NanoPlatelets (GNP) reinforced Tantalum Carbide (TaC) composites are successfully synthesized using Spark Plasma Sintering (SPS) processing method. GNP demonstrate the ability to survive when processed under the extreme conditions needed to synthesize an ultra high temperature ceramic such as $\mathrm{TaC}$. TaCGNP composites are synthesized using SPS at extreme conditions of $1850 \mathrm{C}$ and 100 MPa enabling attainment of high densification with suppressed grain growth. Mechanical properties of TaC-GNP composites are evaluated and the addition of GNP is shown to increase the fracture toughness. Oxidation studies are carried out at extreme conditions using a high temperature plasma flame and the addition of GNP is shown to enhance oxidation resistance. The specific outcomes of reinforcing TaC with GNP are presented below:

GNP are consolidated into a bulk structure demonstrating their ability to survive and retain their structure during extreme processing parameters of $1850{ }^{\circ} \mathrm{C}$ and $100 \mathrm{MPa}$.

Bulk GNP structure reveals inherent toughening mechanisms in the form of sharp angle bending and interlayer platelet sliding. 
GNP are disbursed into TaC matrix with varying effectiveness - TaC-3G reveals highly networked GNP structure. TaC-1G shows insufficient GNP presence, while TaC-5G reveals regions with large GNP agglomerates.

GNP increase uniformity and densification of TaC-GNP compacts while simultaneously suppressing grain growth through GNP grain wrapping mechanisms.

Sheet pullout along with kinking and shearing of GNP indicate an effective transfer of load takes place. These mechanisms dissipate energy thus reducing energy available for fracturing and crack propagation.

Addition of GNP improves fracture toughness by up to $100 \%$. GNP induced toughening mechanisms such as crack deflection and crack bridging are observed during indentation experiments.

Addition of GNP promotes increased thermal conductivity in TaC-GNP composites which reduces heating intensity by dissipating heat throughout structure.

GNP suppresses oxide layer formation by inhibiting diffusion of oxygen into the structure by sealing grain boundaries and by chemically reacting with $\mathrm{Ta}_{2} \mathrm{O}_{5}$ to revert it back to $\mathrm{TaC}$. 


\section{CHAPTER VI: FUTURE RESEARCH}

Based on the conclusions of this research several recommendations for future research can be made. It was seen from the consolidation results that the degrees of densification in this sample were very different from those obtained in previous studies that employed the same processing conditions. While the powder processing was slightly different, the most influential aspect was likely the varying thickness. SPS as a technique has the limitation of producing homogenous and consistent results when size is scaled upward - thicker sample will have less uniformity and less densification. With the advances in joining of UHTCs (e.g. spark plasma joining forms seamless joints) it is longer necessary to synthesize thicker samples. Thinner samples of high density can simply be joined afterwards to form UHTCs are both very thick and very dense. Though joining techniques one could form UHTC rods several centimeters in length. These rods could allow for machining of large specimens needed for advanced mechanical characterization such as notched beam method fracture toughness, flexural testing, and fatigue testing.

The ability of GNP to improve toughness and oxidation behavior necessitates further research. The addition of an additional carbon allotrope such as CNT may further enhance properties. The confluence of GNP and CNT may lead to novel microstructures and interactions between $\mathrm{TaC}$, GNP, and CNT. CNT have shown potential in improving toughness as well as strength in $\mathrm{TaC}$. The CNT could also provide the carbon source needed to attempt to induce localized reducing environments during high temperature exposures. The addition of CNT and GNP may simultaneously refine grains, increase densification, improve oxidation resistance, and enhance strength and toughness. 


\section{LIST OF REFERENCES}

[1] Defense Advanced Research Projects Agency (DARPA). (April 20 ${ }^{\text {th }}$, 2012). Engineering Review Board Concludes Review of HTV-2 Second Flight Test [Press Release]. Retrieved from http://www.darpa.mil/NewsEvents/Releases/2012/04/20.aspx

[2] Squire, T.H., Marschall, J., "Material Property requirements for analysis and design of UHTC components in hypersonic applications", Journal of the European Ceramic Society (2010), Vol. 30, pp. 2239-2251

[3] Bertin, J.J., Cummings, R.M., "Critical Hypersonic Aerothermal Phenomena", Annual Review Fluid Mechanics (2006), Vol 38

[4] Darwent, B.D., "Bond Dissociation Energies in Simple Molecules", United States Department of Commerce National Bureau of Standards (1970), NSRDS-NBS31

[5] Glade, D., "Unmanned Aerial Vehicles: Implications for Military Operations", Air War College Center for Strategy and Technology Occasional Paper No. 16, July (2000)

[6] Washburn, A., Kress, M., "Combat Modeling", Ch.9: Unmanned Aerial Vehicles, International Series in Operations Research and Management Science 134, Springer Science + Business Media, (2009)

[7] Derman, G., "Virtual Instrument Pilot: An Improved Method and System for Navigation and Control of Fixed Wing Aircraft", United States Patent 6,405,107 B1, June $11^{\text {th }}, 2002$

[8] Lin, S.-C., Shen, M.-C., "Flight Simulation of a WaveRider-Based Hypersonic Vehicle", Computers \& Fluids (1997), Vol. 26, No. 1

[9] Hirschberg, M., Hart, D., Beatner, T., "A Summary of a Half-Century of Oblique Wing Research", AIAA Paper 2007-150 (2007)

[10] Nieto, A., Perez, K., Rojas, O., Rodriguez, O., Salman, S., Zha, G.-C., "Towards High Efficiency Hypersonic Flight - Hypersonic Bi-Directional Flying Wing", AIAA Paper 2012-0398 (2012)

[11] Brossierm P.L., Mazeaud, G., Wurniesky, P.C., "Turbojet-Ramjet Hypersonic Aircraft Engine", United States Patent 5,284,014, February ${ }^{\text {th }}, 1994$

[12] Murray, J.J., Hempshell, C.M., Bond, A., "An Experimental Precooler for Airbreathing Rocket Engines", Journal of the British Interplanetary Society (2001), Vol 54, pp. 199-209

[13] Harwood, W., "Boeing, SpaceX, Sierra Nevada win manned spacecraft contracts", CBS News (August $3^{\text {rd }}, \quad$ 2012), Retrieved from http://www.cbsnews.com/network/news/space/home/spacenews/files/080312_ccicap.htm $\underline{1}$ 
[14] NASA, "Fiscal Year 2013 Complete Budget Estimates", (2012), Retrieved from http://www.nasa.gov/news/budget/index.html

[15] Space Exploration Technologies Corportation (SpaceX). (December, $23^{\text {rd }}, 2008$ ). NASA Selects SpaceX's Falcon 9 Booster and Dragon Spacecraft for Cargo Resupply Service to the International Space Station [Press Release]. Retrieved from http://www.spacex.com/press.php?page=20081223

[16] Milos, F.S., Chen, Y.-K., "Ablation and Thermal Response Property Model Validation for Phenolic Impregnated Carbon Ablator", Journal of Spacecraft and Rockets (2010), Vol. 47, No. 5

[17] Elvis, M., "Let's Mine Asteroids - For Science and Profit", Nature (2012), Vol. 485, pp. 549

[18] Mueller, R.P., Van Susante, P.J., "A Review of Extra-Terrestrial Mining Robot Concepts", NASA Kennedy Space Center - Space Processing (2012), Document ID: 20120008777

[19] Canan, J.W., "Breathing new hope into Hypersonics", Aerospace America (2007), Vol. 11, pp. 26-31

[20] Moses, P.L., Rausch, V.L., Nguyen, L.T., Hill, J.R., "NASA hypersonic flight demonstrators - overview, status, and future plans", Acta Astronautica (2004), Vol. 55, pp. 619-630

[21] Dodd, S.P., Cankurtaran, M., James, B., "Ultrasonic determination of the elastic and nonlinear acoustic properties of transition-metal carbide ceramics: $\mathrm{TiC}$ and $\mathrm{TaC}$ ", Journal of Materials Science 38 (2003), 1107-1115

[22] Hackett, K., Verhoef, S, Cutler, R.A., Shetty, D.K., "Phase Constitution and Mechanical properties of Carbides in the Ta-C System", Journal of the American Ceramic Society (2009), 92, pp. 2404-2407

[23] Chen, Y.-J., Li, J.-B., Wei, Q.-M., Zhai, H.-Z., "Preparation and growth mechanism of TaCx whiskers", Journal of Crystal Growth 224 (2001), 244-250

[24] Kim, B.-R., Woo, K.-D., Doh, J.-M., "Mechanical properties and rapid consolidation of binderless nanostructured tantalum carbide", Ceramics International 35 (2009), 33953400

[25] Lee, C., Wei, X., Kysar, J.W., Hone, J. "Measurement of the Elastic Properties and Intrinsic Strength of Monolayer Graphene", Science Volume 321 (2008), Issue 5887, pp.385

[26] Frank, I.W., Tanenbaum, D.M., Van der Zande, A.M., McEuen, P.L., "Mechanical Properties of Suspended Graphene Sheets", Journal of Vacuum Science Technologies (2007) 
[27] XG Sciences, "xGnP® Graphene Nanoplatelets Carbon Nanoparticles with Multifunctional Capability", XG Sciences Documentation (2009)

[28] Ragulya, A.V., "Consolidation of ceramic nanopowders", Advances in Applied Ceramics (2008), Vol 107 No 3 118-134

[29] Bale, C., Chartrand, P., Degterov, S.A., Eriksson, G., Hack, K., Ben Mahfoud, R., Melancon, J., Pelton, A.D., Petersen, S., "Calphad-Comput. Coupling Phase Diagrams", Thermochem. (2002), Vol. 26, pp. 189-228

[30] Fahrenholtz, W.G., Hilmas, G.E., Talmy, I.G., Zaykoski, J.A., "Refractory Diborides of Zirconium and Hafnium", Journal of the American Ceramic Society 90 (2007), 13471364

[31] Post, B., Glaser, F.W., Moskowitz, D., "Transition Metal Diborides", Acta Metallurgica 2 (1954), pp.20-25

[32] Liu, L., Liu, H., Ye, F., Zhang, Z., Zhou, Y., "Microstructure and mechanical properties of the spark plasma sintered $\mathrm{Ta}_{2} \mathrm{C}$ Ceramics", Ceramics International 38 (2012), 4707-4713

[33] Wang, H., Lee, S.-H., Kim, H.-D., "Nano-Hafnium Diboride Powders synthesized using a spark plasma sintering apparatus", Journal of the American Ceramic Society (2012), 1-4

[34] Zamora, V., Ortiz, A.L., Guiberteau, F., Nygren, M., "Spark-plasma sintering of $\mathrm{ZrB}_{2}$ ultra-high-temperature ceramics at lower temperature via nanoscale crystal refinement", Journal of the European Ceramic Society 32 (2012), pp.2529-2536

[35] Wang, H., Fan, B., Feng, L., Chen, D., Lu, H., Xu, H., Wang, C.-A., Zhang, R., “The fabrication and mechanical properties of $\mathrm{SiC} / \mathrm{ZrB}_{2}$ laminated ceramic composite prepared by spark plasma sintering", Ceramics International 38 (2012), 5015-5022

[36] Watts, J., Hilmas, GE, Fahrenholtz, W.G., Brown, D., Clausen, B., "Stress measurements in $\mathrm{ZrB}_{2}-\mathrm{SiC}$ composites using Raman spectroscopy and neutron diffraction", Journal of the American Ceramic Society 30 (2010), 2165-2171

[37] Zou, J., Zhang, G.-J., Hu, C.-F., Nishimura, T., Sakka, Y., Tanaka, H., Vleugels, J., Van der Biest, O., "High-temperature bending strength, internal friction and stiffness of $\mathrm{ZrB}_{2}-20 \mathrm{vol} \% \mathrm{SiC}$ ceramics", Journal of the European Ceramic Society 32 (2012), 25292527

[38] Wang, H., Wang, C.A., Yao, X., Fang, D., "Processing and mechanical properties of zirconium diboride-based ceramics prepared by spark plasma sintering", Journal of the American Ceramic Society 90 (2007), 1992-1997 
[39] Silvestroni, L., Sciti, D., Guicciardi, S., Melandri, C., "Toughened ZrB 2 -based ceramics with addition of $\mathrm{SiC}$ whiskers or chopped fibers", Journal of the European Ceramic 30 (2010), 2155-2164

[40] Sciti, D., Silvestroni, L., "Processing, sintering and oxidation behavior of SiC fibers reinforced Z ZrB 2 composites", Journal of the European Ceramic Society 32 (2012), 19331940

[41] Guicciardi, S., Silvestroni, L., Nygren, M., Sciti, D., "Microstructure and toughening mechanisms in spark plasma sintered $\mathrm{ZrB}_{2}$ ceramics reinforced by $\mathrm{SiC}$ whiskers or $\mathrm{SiC}$ chopped fibers", Journal of the American Ceramic Society 93 (2010), Vol. 8, 2384-2391

[42] Yang, F., Zhang, X., Han, J., Du, S., "Characterization of hot-pressed short carbon fiber reinforced $\mathrm{ZrB}_{2}-\mathrm{SiC}$ ultra-high temperature ceramic composites", Journal of Alloys \& Compunds 472 (2009), 395-9

[43] Ni, D.-W., Liu, J.X., Zhang, G.-J., "Pressureless sintering of $\mathrm{HfB}_{2}-\mathrm{SiC}$ ceramics doped with WC", Journal of the American Ceramic Society 32 (2012), 3627-2635

[44] Carney, C.M., Oxidation resistance of hafnium diboride-silicon carbide from 1400 to $2000{ }^{\circ} \mathrm{C} "$, Journal of Materials Science 44 (2009), 5673-6578

[45] Brown-Shaklee, H.J., Fahrenholtz, W.G., Hilmas, G.E., "Densification behavior and thermal properties of Hafnium Diboride with the addition of Boron Carbides", Journal of the American Ceramic Society 95 (2012), Vol. 6, 2035-2043

[46] Liu, H., Liu, L., Ye, F., Zhang, Z., Zhou, Y., "Microstructure and mechanical properties of the spark plasma sintered $\mathrm{TaC} / \mathrm{SiC}$ composites: Effects of sintering temperatures", Journal of the European Ceramic Society 32 (2012), 3617-3625

[47] Zhang, X., Hilmas, G.E., Fahrenholtz, W.G., "Densification and mechanical properties of TaC-based ceramics", Materials Science and Engineering A 501 (2009), 3743

[48] Zhang, X., Hilmas, G.E., Fahrenholtz, W.G., "Densification, mechanical properties, and oxidation resistance of TaC-TaB2 ceramic", Journal of the American Ceramic Society 91 (2009), Vol. 12, 4129-4132

[49] Sciti, D., Guicciardia, S., Nygren, M., "Spark plasma sintering and mechanical behavior of ZrC-based composites”, Scripta Materialia 59 (2008), 638-641

[50] Ran, S., Huang, S.G., Van der Biest, O., Vleugels, J., "High-strength $\mathrm{ZrB}_{2}$-based ceramics prepared by reactive pulsed electric current sintering of $\mathrm{ZrB}_{2}-\mathrm{ZrH}_{2}$ powders", Journal of the European Ceramic Society 32 (2012), 2537-2543

[51] Liu, L.M., Ye, F., Zhou, Y., "New route to densify tantalum carbide at $1400{ }^{\circ} \mathrm{C}$ by spark plasma sintering”, Materials Science and Engineering A 528 (2011), 4710-4714 
[52] Balani, K., Gonzalez, G., Agarwal, A., "Synthesis, microstructural characterization, and mechanical property evaluation of vacuum plasma sprayed tantalum carbide", Journal of the American Ceramic Society 89 (2006), Vol.4, 1419-1425

[53] Corral, E.L., Loehman, R.E., "Ultra-high-temperature ceramic coatings for oxidation protection of carbon-carbon composites", Journal of the American Ceramic Society 91 (2008), Vol.5, 1495-1502

[54] Mishra, S.K., Das, S., Pathak, L.C., "Defect structures in zirconium diboride powder prepared by self-propagating high-temperature synthesis", Materials Science and Engineering A 364 (2004), 249-255

[55] Chen, L., Gu, Y., Shi, L., Yang, Z., Ma, J., Qian, Y., "Synthesis and oxidation of nanocrystalline $\mathrm{HfB}_{2}$ ", Journal Alloys and Compounds 368 (2004), Vol.1-2, 353-356

[56] Ma, J., Du, Y., Wu, M., Pan, M., "One simple synthesis route to nanocrystalline tantalum carbide via the reaction of tantalum pentachloride and sodium carbonate with metallic magnesium", Materials Letters 61 (2007), 3658-3661

[57] Zamora, V., Ortiz, A.L., Guiberteau, F., Shaw, L.L., "Crystallite size refinement $\mathrm{ZrB}_{2}$ by high-energy ball milling", Journal of the European Ceramic Society 32 (2012), Vol.2, 271-276

[58] Sciti, D., Silvestroni, L., Guicciardi, S., Fabriche, D.D., Bellosi, A., "Processing, mechanical properties and oxidation behavior of $\mathrm{TaC}$ and $\mathrm{HfC}$ composites containing 15 vol\% $\mathrm{TaSi}_{2}$ or $\mathrm{MoSi}_{2}$ ", Journal of the Materials Research Society (2009), Vol.24, No.6, 2056-2065

[59] Zhang, X., Hilmas, G.E., Fahrenholtz, W.G., "Hot pressing of tantalum carbide with and without sintering additives", Journal of the American Ceramic Society 90 (2007), $393-401$

[60] Desmaison-Brut, M., Alexandre, N., Desmaison, J., "Comparison of oxidation behavior of two dense hot isostatically pressed tantalum carbide $\left(\mathrm{TaC}\right.$ and $\left.\mathrm{Ta}_{2} \mathrm{C}\right)$ materials", Journal of the European Ceramic Society 17 (1997), 1325-1334

[61] Levine, S.R., Opila, E.J., Halbig, M.C., Kiser, J.D., Singh, M., Salem, J.A., "Evaluation of ultra-high temperature ceramics for aeropropulsion use", Journal of the European Ceramic Society 22 (2002), 2757-2767

[62] Guo, S., Kagawa, Y., Nishimura, T., Tanaka, H., "Thermal and electrical properties in hot-pressed $\mathrm{ZrB}_{2}-\mathrm{MoSi}_{2}-\mathrm{SiC}$ composites", Journal of the American Ceramic Society 90 (2007), 2255-2258

[63] Sciti, D., Brach, M., Bellosi, A., "Oxidation behavior of a pressureless sintered $\mathrm{ZrB}_{2}-\mathrm{MoSi}_{2}$ ceramic composite", Journal of Materials Research 20 (2005), 922-930 
[64] Opila, E., Levine, S., Lorincz, J., "Oxidation of $\mathrm{ZrB}_{2}$-and $\mathrm{HfB}_{2}$-based ultra-high temperature ceramics: effect of Ta additions", Journal of Materials Science 39 (2004), 5969-6977

[65] Talmy, I.G., Zaykoski, J.A., Opeka, M.M., "Synthesis, processing and properties of

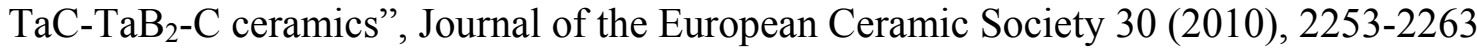

[66] Liu, J.-X., Kan, Y.-M., Zhang, G.-L., "Pressureless sintering of tantalum carbide ceramics without additives", Journal of the American Ceramic Society 93 (2010), 370373

[67] Khaleghi, E., Lin, Y.-S., Meyers. M.A., Olevsky, E.A., "Spark plasma sintering of tantalum carbide", Scripta Materialia 63 (2010), 577-580

[68] Bakshi, S.R., Musaramthota, V., Lahiri, D., Singh, V., Seal, S., Agarwal, A., "Spark plasma sintered tantalum carbide: Effect of pressure and nano-boron carbide addition on microstructure and mechanical properties", Materials Science and Engineering A 528 (2011), 1287-1295

[69] Bakshi, S.R., Musaramthota, V., Virzi, D.A., Keshri, A.K., Lahiri, D., Singh, V., Seal, S., Agarwal, A., "Spark plasma sintered tantalum carbide-carbon nanotube composite: Effect of pressure, carbon nanotube length and dispersion technique on microstructure and mechanical properties", Materials Science and Engineering A 528 (2011), 2538-47

[70] Rao, C.N.R., Sood, A.K., Voggu, R., Subrahmanyam, K.S., "Some Novel Attributes of Graphene", The Journal of Physical Chemistry Letters 1 (2010), 572-580

[71] Fernandez-Mohan, H., "Single Crystals of graphite and mica as specimen support for electron microscopy", Journal of Applied Physics (1960), Vol. 31, 1840

[72] Soldano, C., Mahmood, A., Dujardin, E., "Production, properties, and potential of graphene", Carbon 48 (2010), 2127-2150

[73] Huc, V., Bendiab, N., Rosman, N., Ebbesen, T., Delacour, C., Bouchiat, V., "Large and flat graphene flakes produced by epoxy bonding and reverse exfoliation of highly oriented pyroltic graphite", Nanotechnology 19 (2008)

[74] Ohta, T., Bostwick, A., Seyller, T., Horn, K., Rotenberg, E., "Controlling the electronic structure of bilayer graphene", Science (2006), 951-954

[75] Charrier, A., Coati, A., Argunova, T., Thibaudau, F., Garreau, Y., Pinchaux, R., "Solid State decomposition of $\mathrm{SiC}$ for growing ultra-thin heteroepitaxial graphite films", Journal of Applied Physics (2002), 2479-2484

[76] Malesevic, A., Kemps, R., Zhang, L., Erni, R., Van Tendeloo, G., Vanhulsel, A., Van Haesendonck, C., "A versatile plasma tool for the synthesis of carbon nanotubes and few-layer graphene sheets", Journal of Optoelectronic Advanced Materials 10 (2008) 
[77] Enoki, T., "Diamond-to-graphite conversion in nanodiamond and the electronic properties of nanodiamond-derived carbon system", Physics Solid State (2004), 651-56

[78] Cano-Marquez, A.G., Rodriguez-Macias, F.J., Campos-Delgado, J., EspinosaGonzales, C.G., Tristan-Lopez, F., Ramire-Gonzalez, D., Cullen, D.A., "Ex-MWCNTs: graphene sheets and ribbons produced by lithium intercalation and exfoliation of carbon nanotubes", Nano Letters 9 (2009), 1527

[79] Kosynkin, D.V., Higginbotham, A.L., Sinitskii, A., Lomeda, J.R., Dimiev, A., Price, B.K., Tour, J.M., "Longitudinal unzipping of carbon nanotubes to form graphene nanoribbons", Nature 458 (2009), 872

[80] Jiao, L., Zhang, L., Wang, X., Diankov, G., Dai, H., "Narrow graphene nanoribbons from carbon nanotubes", Nature 458 (2009), 877

[81] Choi, W., Lahiri, I., Seelaboyina, R., Kang, Y.S., "Synthesis of Graphene and its Applications: A Review", Critical Reviews in Solid State and Materials Sciences 35 (2010), 52-71

[82] Kaplas, T., Sharma, D., Svirko, Y., "Few-layer graphene synthesis on a dielectric substrate", Carbon 50 (2012), 1503-1509

[83] Wassei, J.K., Mecklenburg, M., Torres, J.A., Dowler, J.D., Regan, B.C., Kaner, R.B., Weiller, B.H., "Chemical Vapor Deposition of Graphene on Copper from Methane, Ethane, and Propane: Evidence for Bilayer Selectivity”, Small 8 (2012), No.9, 1415-22

[84] Zou, R., Zhang, Z., Xu, K., Jiang, L., Tian, Q., Sun, Y., Chen, Z., Hu, J., “A method for joining individual graphene sheets", Carbon 50 (2012), 4965-4972

[85] Ferrari, A.C., Meyer, J.C., Scardaci, V., Casiraghi, C., Lazzeri, M., Piscanec, S., "Raman spectrum of graphene and graphene layers", Physical Review Letters 97 (2006), $187-201$

[86] Dresselhaus, M.S., Jorio, A., Hofmann, M., Dresselhaus, G., Saito, R., "Perspectives on carbon nanotubes and graphene Raman spectroscopy", Nano Letters 10 (2010), 75158

[87] Kalbac, M., Farhat, H., Kong, J., Janda, P., Kavan, L., Dresselhaus, M.S., Raman spectroscopy and in situ raman spectroelectrochemistry of bilayer 12C/13C graphene", Nano Letters 11 (2011), 1957-63

[88] Ferrari, A.C., "Raman spectroscopy of graphene and graphite: Disorder, electronphonon coupling, doping and nonadiabatic effects", Solid State Communications 143 (2007), 47-57

[89] Ni, Z.H., Wang, H.M., Ma, Y., Kasim, J., Wu, Y.H., Shen, Z.X., "Tunable stress and controlled thickness modification in graphene by annealing", ACS Nano 2 (2008), 103339 
[90] Tsoukleri, G., Parthenios, J., Papagelis, K., Jalil, R., Ferrari, A.C., Geim, A.K., Novoselov, K.S., Galiotis, C., "Subjecting a graphene monolayer to tension and compression", Small 5 (2009), No. 21, 2397-2402

[91] Gibson, J.O., Gibson, M.G., "Production of carbon fiber-tantalum carbide composites", United States Patent 4,196,230, April 1 ${ }^{\text {st }}, 1980$

[92] Bakshi, S.R., Lahiri, D., Agarwal, A., "Carbon nanotube reinforced metal matrix composites - a review”, International Materials Reviews 55 (2010), 41-64

[93] Kim, P., Shi, L., Majumdar, A., McEuen, P.I., “Thermal Transport Measurements of Individual Multiwalled Nanotubes”, Physical Review Letters 87 (2001), No.21

[94] Shi, X.L., Yang, H., Shao, G.Q., Duan, X.L., Yan, L., Xiong, Z., Sun, P., "Fabrication and properties of W-Cu alloy reinforced by multi-walled carbon nanotubes", Materials Science and Engineering A 457 (2007), 18-23

[95] Bakshi, S.R., Agarwal, A., "An analysis of the factors affecting strengthening in carbon nanotube reinforced aluminum composites", Carbon 49 (2011), 533-544

[96] Coleman, J.N., Khan, U., Blau, W.J., Gun'ko, Y.K., "Small but strong: a review of the mechanical properties of carbon nanotube-polymer composites", Carbon 44 (2006), $1624-1652$

[97] Yu, M.F., Lourie, O., Dyer, M.J., Moloni, K., Kelly, T.F., Ruoff, R.S., "Strength and breaking mechanism of multiwalled carbon nanotubes under tensile load" Science 287 (2000), 637-640

[98] He, C., Zhao, N., Shi, C., Du, X., Li, J., Li, H., Cui, Q., “An approach to obtaining homogeneously dispersed carbon nanotubes in Al powders for preparing reinforced Almatrix composites", Advanced Materials 19 (2007), 1128-1132

[99] Lahiri, D., Singh, V., Keshri, A.K., Seal, S., Agarwal, A., "Carbon nanotube toughened hydroxyapatite by spark plasma sintering: Microstructural evolution and multiscale tribological properties", Carbon 48 (2010), 3103-3120

[100] Nakahira, A., Eguchi, K., "Evaluation of microstructure and some properties of hydroxyapatite/Ti composites", Journal of Ceramic Processing Research 2 (2003), No.3, $108-112$

[101] Xu, C., Wang, X., Zhu, J., “Graphene-metal particle nanocomposites”, Journal of Physical Chemistry 112 (2008), 19841-5

[102] Lahiri, D., Agarwal, A., "Graphene reinforced ceramic and metal matrix composites" In: Choi, W., Lee, J., editors. "Graphene: Synthesis and applications”, Boca Raton: CRC Press; (2012), pp. 187-232 
[103] Chen, L.-Y., Konishi, H., Fehrenbacher, A., Ma, C., Xu, J.-Q., Choi, H., Xu,H.-F., Pfefferkorn, F.E., Li, X.-C., "Novel nanoprocessing route for bulk graphene nanoplatelets reinforced metal matrix nanocomposites", Scripta Materialia 67 (2012), pp. 27-32

[104] Fan, Y., Wang, L., Li, J., Li, J., Sun, S., Chen, F., Chen, L., Jiang, W., "Preparation and electrical properties of graphene nanosheet/A12O3 composites", Carbon 48 (2010), 1743-1749

[105] He, T., Li, J., Wang, L., Zhu, J., Jiang, W., "Preparation and consolidation of alumina/graphene composite powders", Materials Transactions 50 (2009), No.4, pp.749751

[106] Wang, K., W., Y., Fan, Z., Yan, J., Wei, T., "Preparation of graphene nanosheet/alumina composites by spark plasma sintering", Materials Research Bulletin 46 (2011), Issue 2, 315-318

[107] Walker, L.S., Marotto, V.R., Rafiee, M.A., Koratkar, N., Corral, E.L., "Toughening in graphene ceramic composites", ACS Nano 5 (2011), No. 4, 3182-3190

[108] Ramirez, C., Figueiredo, F.M., Miranzo, P., Poza, P., Osendi, M.I., "Graphene nanoplatelets/silicon nitride composites with high electrical conductivity", Carbon 50 (2012), 3607-3615

[109] Dusza, J., Morgiel, J., Duszova, A., Kvetkova, L., Nosko, M., Kun, P., Balazsi, C., "Microstructure and fracture toughness of Si3Ni4+graphene platelet composites", Journal of the European Ceramic Society 32 (2012), 3389-3397

[110] Balandin, A.A, Ghosh, S., Bao, W., Calizo, I., Teweldebrhan, D., Miao, F., Lau, C.N., "Superior thermal conductivity of single-layer graphene", Nano Letters 8 (2008), No.3, 902-907

[111] Bolotin, K.I., Sikes, K.J., Jiang, Z., Klima, M., Fudenberg, G., Hone, J., "Ultrahigh electron mobility in suspended graphene", Solid State Communications 146 (2010), 351355

[112] Chou, Y.S., Green, D.J., "Silicon carbide platelet/alumina composites: I, effect of forming technique on platelet orientation", Journal of the American Ceramic Society 75 (1992), 3346-3352

[113] Kellet, B.C., Wilkinson, D.S., "Processing and sintering of alumina-graphite platelet composites", Journal of the American Ceramic Society 78 (1995), 1198-200

[114] Rafiee, M.A., Rafiee, J., Wang, Z., Song, H., Yu, Z.-Z., Koratkar, N., "Enhanced mechanical properties of nanocomposites at low graphene contents", ACS Nano 3 (2009), No.12, pp. 3884-3890 
[115] Munir, Z.A., Anselmi-Tamburini, U., "The effect of electric field and pressure on the synthesis and consolidation of materials: A review of the spark plasma sintering method", Journal of Materials Science 41 (2006), 763-777

[116] Lu, K., "Sintering of nanoceramics", International Materials Reviews 53 (2008), No. 1, pp.21-38

[117] Ragulya, A.V., "Fundamentals of Spark Plasma Sintering", Encyclopedia of Materials: Science and Tecnology (2010), pp.1-5

[118] Sciti, D., Guicciardi, S., Nygren, M., "Densification and mechanical behavior of $\mathrm{HfC}$ and $\mathrm{HfB}_{2}$ fabricated by spark plasma sintering", Journal of the American Ceramic Society 91 (2008), No.5, 1433-1440

[119] Olevsky, E.A., Bradbury, W.L., Haines, C.D., Martin, D.G., Kapoor, D., "Fundamental Aspects of Spark Plasma Sintering: I. "Fundamental Aspects of Spark Plasma Sintering: I. Experimental Analysis of Scalability", Journal of the American Ceramic Society 95 (2012), Issue 8, pp. 2406-2413

[120] Olevsky, E.A., Garcia-Cardona, C., Bradbury, W.L., Haines, C.D., Martin, D.G., Kapoor, D., "Fundamental Aspects of Spark Plasma Sintering: II. Finite Element Analysis of Scalability", Journal of the American Ceramic Society 95 (2012), Issue 8, pp. 2414-2422

[121] Shen, J., Zhang, F.M., Sun, J.F., Zhu, Y.Q., McCartney, D.G., "Spark Plasma sintering assisted diamond formation from carbon nanotubes at very low pressure", Nanotechnology 17 (2006), 2187-2191

[122] Yang, K., He, J., Su, Z., Reppert, J.B., Skove, M.J., Tritt, T.M., Rao, A.M., “Intertube bonding, graphene formation and anisotropic transport properties in spark plasma sintered multi-wall carbon nanotube arrays”, Carbon 48 (2010), 756-762

[123] Laurent, C., Chevallier, G., Weibel, A., Peigney, A., Estournes, C., "Spark plasma sintering od double-walled carbon nanotubes", Carbon 46 (2008), 1792-1828

[124] Li, J.L., Wang, L.J., He, T., Jiang, W., "Surface graphitization and mechanical properties of hot-pressed bulk carbon nanotubes compacted by spark plasma sintering", Carbon 45 (2007), 2636-2642

[125] Hu, P., Guolin, W., Wang, Z., "Oxidation mechanism and resistance of $\mathrm{ZrB}_{2}-\mathrm{SiC}$ composites", Corrosion Science 51 (2009), 2742-2731

[126] Shi, G., Wang, Z., Sun, X., Wu, Z., "Effect of the surface oxidation on the flexural strength of the $\mathrm{ZrB}_{2}-\mathrm{SiC}-\mathrm{ZrC}$ ceramic", Materials Science and Engineering A 546 (2012), $162-168$ 
[127] Wu, Z., Wang, Z., Shi, G., Sheng, J., "Effect of surface oxidation on thermal shock resistance of the $\mathrm{ZrB}_{2}-\mathrm{SiC}-\mathrm{ZrC}$ ceramic", Composites Science and Technology 71 (2011), 1501-1506

[128] Zhi, W., Qiang, Q., Zhanjun, W., Guodong, S., "Effect of oxidation at $1100{ }^{\circ} \mathrm{C}$ on the strength of $\mathrm{ZrB}_{2}$-SiC-graphite ceramics", Journal of Alloys and Compounds 509 (2011), 6871-6875

[129] Monteverde, F., "Ultra-High Temperature HfB2-SiC Ceramics Consolidated by Hot-Pressing and Spark Plasma Sintering”, Journal of Alloys and Compounds 428 (2007), 197-205

[130] Monteverde, F., "Progress in the Fabrication of Ultra-High-Temperature Ceramics: "In Situ" Synthesis, Microstructure and Properties of a Reactive Hot-Pressed $\mathrm{HfB}_{2}-\mathrm{SiC}$ Composite", Composite Science and Technology 65 (2005), 1869-1879

[131] Monteverde, F., Melandri, C., Guicciardi, S., "Microstructure and Mechanical Properties of an $\mathrm{HfB}_{2}+30$ vol\% $\mathrm{SiC}$ Composites Consolidated by Spark Plasma Sintering", Materials Chemistry and Physics 100 (2006), 513-519

[132] Sciti, D., Silvestroni, L., Bellosi, A., "Fabrication and Properties of $\mathrm{HfB}_{2}-\mathrm{MoSi}_{2}$ Composites Produced by Hot Pressing and Spark Plasma Sintering", Journal of Materials Research 21 (2006), 1460-61

[133] Bellosi, A., Monteverde, F., Sciti, D., "Fast densification of Ultra-High Temperature Ceramics by Spark Plasma Sintering", International Journal of Applied Ceramic Technology 3 (2006), 32-40

[134] Marschall, J., Fletcher, D.G., "High-enthalpy test environments, flow modeling and in situ diagnostics for characterizing ultra-high temperature ceramics", Journal of the European Ceramic Society 30 (2010), 2323-2336

[135] Zhang, X., Hu, P., Han, J., Meng, S., "Ablation behavior of $\mathrm{ZrB}_{2}-\mathrm{SiC}$ ultra high temperature ceramics under simulated atmospheric re-entry conditions", Composites Science and Technology 68 (2008), 1718-1726

[136] Alfano, S., Scatteia, L., Monteverde, F., Beche, E., Balat-Pichelin, M., "Microstructural characterization of $\mathrm{ZrB}_{2}$-SiC based UHTC tested in the MESOX plasma facility", Journal of the European Ceramic Society 30 (2010), 2345-2355

[137] Monteverde, F., Savino, R., Fumo, M.D.S., Maso, A.D., "Plasma wind tunnel testing of ultra-high temperature $\mathrm{ZrB}_{2}$ - $\mathrm{SiC}$ composites under hypersonic re-entry conditons", Journal of the European Ceramic Society 30 (2010), 2312-2321

[138] Sciti, D., Savino, R., Silvestroni, L., "Aerothermal behavior of a SiC fibrereinforced $\mathrm{ZrB}_{2}$ sharp component in supersonic regime", Journal of the European Ceramic Society 32 (2012), 1837-1845 
[139] Monteverde, F., Savino, R., " $\mathrm{ZrB}_{2}$-SiC Sharp Leading Edges in High Enthalpy Supersonic Flows", Journal of the American Ceramic Society 95 (2012), Issue 7, 2282-9

[140] Savino, R., Fumo, M.D.S., Silvestroni, L., Sciti, D., "Arc-jet testing on $\mathrm{HfB}_{2}$ and HfC-based ultra-high temperature ceramic materials", Journal of the European Ceramic Society 28 (2008), 1899-1907

[141] Wang, Y.-L., Xiong, X., Li, G.-D., Liu, H.-F., Chen, Z.-K., Sun, W., Zhao, X.-J., "Ablation behavior of $\mathrm{HfC}$ protective coating for carbon/carbon composites in oxyacetylene combustion flame", Corrosion (2012), DOI: http://dx.doi.org/10.1016/j.corsci.2012.08.064

[142] Bao, M., Lahiri, D., Keshri, A.K., Zhang, C., Bakshi, S.R., Agarwal, A., "Oxidation behavior of $\mathrm{TaC}$ based Composites in High Temperature Plasma Environment", Journal of the American Ceramic Society (2012), Submitted July 2012

[143] Savino, R., Fumo, M.D.S., Paterna, D., Maso, A.D., Monteverde, F., "Arc-jet testing of ultra-high-temperature-ceramics", Aerospace Science and Technology 14 (2010), 178-187

[144] Tang, S., Deng, J., Wang, S., Liu, W., Yang, K., "Ablation behaviors of ultra-high temperature ceramic composites", Materials Science and Engineering A 465 (2007), 1-7

[145] Anstis, G.R., Chantikul, P., Lawn, B.R., Marschall, D.B., “A Critical Evaluation of Indentation Techniques for Measuring Fracture Toughness: I, Direct Crack Measurements", Journal of the American Ceramic Society 64 (1981), Issue 9, 533-538

[146] Oliver W.C., Pharr G.M., "An improved technique for determining hardness and elastic modulus using load and displacement sensing indentation experiments.", Journal of Materials Research 76 (1992),1564-83.

[147] Keshri, A.K., Patel, R.R., Agarwal, A., "Comprehensive process maps to synthesize high density plasma sprayed aluminum oxide composite coatings with varying carbon nanotube content", Surface Coatings and Technology 205 (2009), 2056-2065

[148] Nieto, A., Lahiri, D., Agarwal, A., "Synthesis and properties of bulk graphene nanoplatelets consolidated by spark plasma sintering", Carbon 50 (2012), 4067-4077

[149] Lopez-de-la-Torre, L., Winkler, B., Schreuer, J., Knorr, K., Avalos-Borja, M., "Elastic properties of tantalum Carbide (TaC), Solid State Communications 134 (2005), $245-250$

[150] Wuchina, E., Opila, E., Opeka, M., Fahrenholtz, W., Talmy, I., "UHTCs: UltraHigh Temperature Ceramic Materials for Extreme Environement Applications", The Electrochemical Society Interface (2007), pp. 30-36 
[151] Gilbert, C.J., Bloyer, D.R., Barsoum, M.W., El-Raghy, T., Tomsia, A.P., Ritchie, R.O., "Fatigue-crack growth and fracture properties of coarse and fine-grained $\mathrm{Ti}_{3} \mathrm{SiC}_{2}$ ", Scripta Materialia 42 (2000), pp.761-767

[152] Sun, Z.-M., Zhang, Z.-F., Hashimoto, H., Abe, T., "Ternary Compound $\mathrm{Ti}_{3} \mathrm{SiC}_{2}$ : Part II. Deformation and fracture behavior at different temperatures", Materials Transactions 43 (2002), No. 3, pp.432-435 